Medical Services

\title{
Standards of Medical Fitness
}

Rapid Action Revision (RAR) Issue Date: 4 August 2011 


\section{SUMMARY of CHANGE}

AR 40-501

Standards of Medical Fitness

This rapid action revision, dated 4 August $2011--$

- Implements the Don't Ask, Don't Tell Repeal Act of 2010 by deleting all references to separation for homosexual conduct (previously in table 8-2).

- Makes administrative changes (app A: removed "-R" from "DA Form 5889-R" in reference forms; marked forms that were not cited in the publication; obsolete publications marked). 


\section{Standards of Medical Fitness}

By Order of the Secretary of the Army:

\section{RAYMOND T. ODIERNO \\ General, United States Army Chief of Staff}

Official:

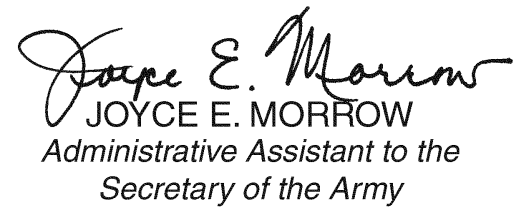

History. This publication is a rapid action revision (RAR). This RAR is effective 20 September 2011. The portions affected by this RAR are listed in the summary of change.

Summary. This publication implements DODD 6130.3 and DODI 6130.4. It provides information on medical fitness standards for induction, enlistment, appointment, retention, and related policies and procedures.

Applicability. This regulation applies to the active Army, the Army National Guard/Army National Guard of the United States, and the U.S. Army Reserve, unless otherwise stated. It also applies to candidates for military service. During mobilization, the proponent may modify chapters and policies contained in this regulation.

Proponent and exception authority. The proponent of this regulation is The Surgeon General. The proponent has the authority to approve exceptions or waivers to this regulation that are consistent with controlling law and regulations. The proponent may delegate this approval authority, in writing, to a division chief within the proponent agency or its direct reporting unit or field operating agency, in the grade of colonel or the civilian equivalent. Activities may request a waiver to this regulation by providing justification that includes a full analysis of the expected benefits and must include formal review by the activities senior legal officer. All waiver requests will be endorsed by the commander or senior leader of the requesting activity and forwarded through their higher headquarters to the policy proponent. Refer to AR 25-30 for specific guidance.

Army management control process. This regulation contains internal control provisions and identifies key internal controls that must be evaluated (see appendix B).

Supplementation. Supplementation of this regulation and establishment of command and local forms are prohibited without prior approval from The Surgeon General (DASG-HS-AS), 5109 Leesburg Pike, Falls Church, VA 22041-3258.

Suggested improvements. Users are invited to send comments and suggested improvements on DA Form 2028 (Recommended Changes to Publications and Blank Forms) directly to the Office of The Surgeon General (DASG-HS-AS), 5109 Leesburg Pike, Falls Church, VA 22041-3258.

Distribution. This publication is available in electronic media only and is intended for command levels A, B, C, D, and $\mathrm{E}$ for medical activities only of the active Army, the Army National Guard/ Army National Guard of the United States, and the U.S. Army Reserve.

Contents (Listed by paragraph and page number)

Chapter 1

General Provisions, page 1

Purpose • 1-1, page 1

References - 1-2, page 1

Explanation of abbreviations and terms $-1-3$, page 1

Responsibilities - 1-4, page 1

Medical classification $\bullet 1-5$, page 1

Review authorities and waivers $\bullet 1-6$, page 1

\section{Chapter 2}

Physical Standards for Enlistment, Appointment, and Induction, page 2

General • 2-1, page 2 


\section{Contents-Continued}

Application and responsibilities - 2-2, page 2

Abdominal organs and gastrointestinal system $-2-3$, page 4

Blood and blood-forming tissue diseases $-2-4$, page 5

Dental $\bullet 2-5$, page 5

Ears - 2-6, page 5

Hearing - 2-7, page 5

Endocrine and metabolic disorders $-2-8$, page 6

Upper extremities $\bullet 2-9$, page 6

Lower extremities $\bullet 2-10$, page 7

Miscellaneous conditions of the extremities $\bullet 2-11$, page 7

Eyes • 2-12, page 8

Vision - 2-13, page 9

Genitalia • 2-14, page 10

Urinary system $\bullet 2-15$, page 11

Head - 2-16, page 11

Neck - 2-17, page 11

Heart • 2-18, page 11

Vascular system $\bullet 2-19$, page 12

Height $\bullet 2-20$, page 12

Weight $・ 2-21$, page 12

Body build $-2-22$, page 12

Lungs, chest wall, pleura, and mediastinum $\bullet 2-23$, page 12

Mouth - 2-24, page 13

Nose, sinuses, and larynx $\bullet 2-25$, page 13

Neurological disorders $\bullet 2-26$, page 13

Learning, psychiatric and behavioral disorders $\bullet 2-27$, page 14

Skin and cellular tissues - 2-28, page 15

Spine and sacroiliac joints - 2-29, page 16

Systemic diseases $\bullet 2-30$, page 17

Tumors and malignant diseases • 2-31, page 17

General and miscellaneous conditions and defects $\bullet 2-32$, page 18

\section{Chapter 3}

Medical Fitness Standards for Retention and Separation, Including Retirement, page 20

General • 3-1, page 20

Application - 3-2, page 21

Disposition - 3-3, page 21

General policy $\bullet 3-4$, page 21

Abdominal and gastrointestinal defects and diseases $\bullet$ 3-5, page 21

Gastrointestinal and abdominal surgery $\bullet 3-6$, page 22

Blood and blood-forming tissue diseases $\bullet$ 3-7, page 22

Dental diseases and abnormalities of the jaws $\cdot 3-8$, page 23

Ears • 3-9, page 23

Hearing • 3-10, page 23

Endocrine and metabolic disorders • 3-11, page 23

Upper extremities $\bullet 3-12$, page 24

Lower extremities $\bullet 3-13$, page 24

Miscellaneous conditions of the extremities $\bullet 3-14$, page 25

Eyes - 3-15, page 25

Vision - 3-16, page 26

Genitourinary system • 3-17, page 26

Genitourinary and gynecological surgery $\bullet 3-18$, page 27

Head • 3-19, page 27

Neck • 3-20, page 27

Heart • 3-21, page 27 


\section{Contents-Continued}

Vascular system $-3-22$, page 28

Miscellaneous cardiovascular conditions $\bullet 3-23$, page 29

Surgery and other invasive procedures involving the heart, pericardium, or vascular system $-3-24$, page 29

Trial of duty and profiling for cardiovascular conditions $-3-25$, page 29

Tuberculosis, pulmonary $\bullet 3-26$, page 30

Miscellaneous respiratory disorders $\bullet 3-27$, page 30

Surgery of the lungs $\bullet 3-28$, page 31

Mouth, esophagus, nose, pharynx, larynx, and trachea $\bullet 3-29$, page 31

Neurological disorders $\cdot 3-30$, page 32

Disorders with psychotic features $-3-31$, page 32

Mood disorders $\bullet$ 3-32, page 32

Anxiety, somatoform, or dissociative disorders $\bullet 3-33$, page 33

Dementia and other cognitive disorders due to general medical condition $\bullet 3-34$, page 33

Personality, psychosexual conditions, transsexual, gender identity, exhibitionism, transvestism, voyeurism, other paraphilias, or factitious disorders; disorders of impulse control not elsewhere classified $\bullet$ 3-35, page 33

Adjustment disorders $\bullet 3-36$, page 33

Eating disorders $\bullet 3-37$, page 33

Skin and cellular tissues $-3-38$, page 33

Spine, scapulae, ribs, and sacroiliac joints $\cdot 3-39$, page 34

Systemic diseases $\cdot 3-40$, page 34

General and miscellaneous conditions and defects $\cdot 3-41$, page 35

Malignant neoplasms $\cdot 3-42$, page 36

Benign neoplasms $\cdot 3-43$, page 36

Sexually transmitted diseases $-3-44$, page 36

Exertional heat illness $\bullet$ 3-45, page 36

Cold injury $\bullet 3-46$, page 37

\section{Chapter 4}

Medical Fitness Standards for Flying Duty, page 40

General $\bullet 4-1$, page 40

Classes of medical standards for flying and applicability $-4-2$, page 40

Aeromedical consultation - 4-3, page 41

Abdomen and gastrointestinal system $-4-4$, page 41

Blood and blood-forming tissue diseases $\cdot 4-5$, page 41

Dental • 4-6, page 41

Ears - 4-7, page 42

Hearing $\bullet 4-8$, page 42

Endocrine and metabolic diseases $-4-9$, page 42

Extremities - 4-10, page 42

Eyes $-4-11$, page 42

Vision - 4-12, page 43

Genitourinary $\bullet 4-13$, page 44

Head and neck $-4-14$, page 44

Heart and vascular system $\cdot 4-15$, page 44

Linear anthropometric dimensions $\cdot 4-16$, page 45

Weight and body build $\cdot 4-17$, page 45

Lung and chest wall $\cdot 4-18$, page 45

Mouth • 4-19, page 45

Nose $\cdot 4-20$, page 45

Pharynx, larynx, trachea, and esophagus $-4-21$, page 46

Neurological disorders $\bullet$ 4-22, page 46

Mental disorders $\bullet 4-23$, page 47

Skin and cellular tissues $-4-24$, page 48

Spine, scapula, ribs, and sacroiliac joints $-4-25$, page 48

Systemic diseases $・ 4-26$, page 48 


\section{Contents-Continued}

Malignant diseases and tumors $-4-27$, page 48

Sexually transmitted diseases $-4-28$, page 48

Aeromedical adaptability $\bullet 4-29$, page 48

Reading Aloud Test $\bullet$ 4-30, page 49

Department of the Army civilian and contract civilian aircrew members $-4-31$, page 49

Medical standards for Class 3 personnel $-4-32$, page 49

Medical standards for ATC personnel $\bullet$ 4-33, page 50

\section{Chapter 5}

Medical Fitness Standards for Miscellaneous Purposes, page 51

General $\bullet 5-1$, page 51

Application - 5-2, page 51

Medical fitness standards for initial selection for Airborne training, Ranger training, and Special Forces training, and

Reconnaissance and Surveillance Leaders Course training - 5-3, page 51

Medical fitness standards for selection for survival, evasion, resistance, escape training $\bullet$ 5-4, page 53

Medical fitness standards for retention for Airborne duty, Ranger duty, and Special Forces duty • 5-5, page 54

Medical fitness standards for initial selection for free fall parachute training $\bullet 5-6$, page 54

Medical fitness standards for retention for free fall parachute duty $\bullet 5-7$, page 55

Medical fitness standards for Army service schools • 5-8, page 56

Medical fitness standards for initial selection for marine diving training (Special Forces and Ranger combat diving) - 5-9, page 56

Medical fitness standards for retention for marine diving duty (Special Forces and Ranger combat diving) • 5-10, page 57

Medical fitness standards for initial selection for other marine diving training (MOS 00B) $-5-11$, page 57

Medical fitness standards for retention for other marine diving duty (MOS 00B) $\bullet 5-12$, page 59

Asplenic Soldiers • 5-13, page 59

Medical fitness standards for deployment and certain geographical areas $\bullet$ 5-14, page 60

Height-U.S. Military Academy, Reserve Officers-Training Corps, and Uniformed Services University of Health Sciences $\cdot 5-15$, page 64

\section{Chapter 6}

Aeromedical Administration, page 64

General $\bullet 6-1$, page 64

Definition of terms $-6-2$, page 64

Application $\cdot 6-3$, page 65

Army Aviation Medicine Program responsibilities $-6-4$, page 65

Authorizations - 6-5, page 66

Classification of flying duty medical exams $\bullet$ 6-6, page 66

Purpose of flying duty medical exams $\bullet 6-7$, page 66

Frequency and period of validity of FDMEs $-6-8$, page 67

Facilities and examiners $-6-9$, page 67

Disposition and review of flying duty medical examinations $-6-10$, page 68

Issuing DA Form $4186 \cdot 6-11$, page 69

General principles $\bullet 6-12$, page 70

Responsibilities and review following a change in health of aircrew members $\bullet 6-13$, page 71

Review and disposition of disqualifications for Class $3 \cdot 6-14$, page 71

Review and disposition of disqualifications for Classes $2 / 2 \mathrm{~F} / 4 \cdot 6-15$, page 72

Temporary medical suspension $\bullet$ 6-16, page 72

Medical termination from aviation service $\bullet 6-17$, page 72

Aeromedical waiver $\bullet$ 6-18, page 73

Aeromedical requalification $\bullet 6-19$, page 73

Waiver and suspension authorities $\bullet$ 6-20, page 74 


\section{Contents-Continued}

\section{Chapter 7}

Physical Profiling, page 75

General $\bullet 7-1$, page 75

Application $\bullet 7-2$, page 75

Physical profile serial system $-7-3$, page 75

Temporary vs. permanent profiles $\cdot 7-4$, page 76

Representative profile serial and codes $\bullet 7-5$, page 77

Profiling officer and approving authority $\bullet$ 7-6, page 77

Recording and reporting of initial physical profile $\cdot 7-7$, page 78

Profiling reviews and approvals $\cdot 7-8$, page 78

Profiling pregnant Soldiers $\bullet 7-9$, page 79

Postpartum profiles $\cdot 7-10$, page 80

Preparation, approval, and disposition of DA Form $3349 \cdot 7-11$, page 81

Responsibility for personnel actions $-7-12$, page 82

Physical profile and the Army Weight Control Program - 7-13, page 82

\section{Chapter 8}

Medical Examinations-Administrative Procedures, page 85

General • 8-1, page 85

Applications $\bullet 8-2$, page 85

Physical fitness $-8-3$, page 85

Consultations $\bullet$ 8-4, page 86

Distribution of medical reports $-8-5$, page 86

Documentary medical evidence $\cdot 8-6$, page 86

Facilities and examiners $\bullet 8-7$, page 87

Hospitalization $\bullet 8-8$, page 87

Objectives of medical examinations - 8-9, page 87

Recording of medical examinations - 8-10, page 87

Scope of medical examinations $\bullet 8-11$, page 87

Medical examination requirements and required forms $\bullet 8-12$, page 88

Report of medical history forms $\bullet$ 8-13, page 90

Validity times for DD Forms $2808 \cdot 8-14$, page 90

Procurement medical examinations $\bullet 8-15$, page 91

Active duty for training, active duty for special work, and inactive duty training $\bullet$ 8-16, page 91

Retiree Recalls • 8-17, page 91

Health Records • 8-18, page 91

Mobilization of units and members of Reserve Components of the Army • 8-19, page 91

Periodic health assessments - 8-20, page 91

Frequency of additional/alternate examinations $\bullet$ 8-21, page 93

Deferment of examinations - 8-22, page 93

Promotion - 8-23, page 94

Separation and retirement examinations $\bullet$ 8-24, page 94

Miscellaneous medical examinations $\bullet$ 8-25, page 96

Cardiovascular Screening Program (CVSP) • 8-26, page 96

Speech Recognition in Noise Test for H3 profile Soldiers • 8-27, page 98

\section{Chapter 9}

Army Reserve Medical Examinations, page 106

General • 9-1, page 106

Application • 9-2, page 106

Responsibility for medical fitness - 9-3, page 106

Examiners and examination facilities - 9-4, page 106

Examination reports • 9-5, page 106

Conduct of examinations - 9-6, page 106

Types of examinations and their scheduling $\bullet$ 9-7, page 106 


\section{Contents-Continued}

Physical profiling $・ 9-8$, page 106

Examination reviews $-9-9$, page 107

Disposition of medically unfit Reservists - 9-10, page 107

Requests for continuation in the USAR - 9-11, page 107

Request for PEB evaluation - 9-12, page 107

Disposition of Reservists temporarily disqualified because of medical defects - 9-13, page 108

Annual dental examinations - 9-14, page 108

\section{Chapter 10}

Army National Guard, page 109

General • 10-1, page 109

Application $\cdot 10-2$, page 109

Medical standards $\bullet 10-3$, page 109

Entry into AGR (Title 10/32) Program • 10-4, page 109

Active duty for more than 30 days (other than Title 10/32 AGR) $10-5$, page 109

Re-entry on active duty or FTNGD - 10-6, page 109

Applications for Federal Recognition - 10-7, page 109

General officer medical examinations $\bullet 10-8$, page 109

Immunizations $\bullet 10-9$, page 110

Periodic medical examinations $\bullet 10-10$, page 110

Waivers $・ 10-11$, page 110

Profiling $\cdot 10-12$, page 110

Individual responsibility $\bullet 10-13$, page 110

Significant incident reporting responsibility $\bullet 10-14$, page 110

Duty restrictions $\cdot 10-15$, page 111

Authorization for examinations $\cdot 10-16$, page 111

Examination authorities $\bullet 10-17$, page 111

Examination review requirements/quality assurance $\cdot 10-18$, page 111

Scope of medical examinations $\bullet 10-19$, page 112

Report of medical examinations $\cdot 10-20$, page 112

Directed examinations $\bullet 10-21$, page 112

Administrative information $\bullet 10-22$, page 112

Special examinations $\cdot 10-23$, page 112

Cardiovascular Screening Program (AGR Soldiers) • 10-24, page 113

Soldiers pending separation for failing to meet medical retention standards $\cdot 10-25$, page 113

Annual dental examination $\cdot 10-26$, page 113

Physical inspections prior to annual training $\bullet 10-27$, page 114

\section{Chapter 11}

Individual Medical Readiness Standards, page 114

General $\bullet 11-1$, page 114

Purpose $\cdot 11-2$, page 114

Responsibilities $\cdot 11-3$, page 114

Individual Medical Readiness elements $\cdot 11-4$, page 115

Individual Medical Readiness categories $-11-5$, page 117

Disposition of Individual Medical Readiness data $111-6$, page 118

\section{Appendixes}

A. References, page 119

B. Management Control Evaluation Checklist, page 126 


\section{Contents-Continued}

\section{Table List}

Table 2-1: Military acceptable weight (in pounds) as related to age and height for males-Initial Army procurement, page 19

Table 2-2: Military acceptable weight (in pounds) as related to age and height for females-Initial Army procurement, page 20

Table 3-1: Methods of assessing cardiovascular disability, page 38

Table 3-2: Profile progression recommendations for the Soldier with heat stroke, with or without Sequelae, complex heat stroke, heat exhaustion, or heat injury, pending a medical evaluation board, page 39

Table 4-1: Acceptable audiometric hearing level for Army aviation and air traffic control, page 51

Table 4-2: Head injury guidelines for Army aviation, page 51

Table 5-1: Guidance on deployment of Soldiers with diabetes, page 61

Table 6-1: Number of months for which a flying duty medical examination (FDME) is valid (Active Component), page 74

Table 7-1: Physical profile functional capacity guide, page 83

Table 7-2: Profile codes, page 84

Table 8-1: Recording of medical examination, page 98

Table 8-2: Schedule of separation medical examination or separation physical assessment, page 103

Table 8-3: Results of Speech Recognition in Noise Test (SPRINT), page 104

Table 8-4: USPSTF Recommendations with Modifications in accordance with this regulation, page 104

\section{Figure List}

Figure 8-1: Normative data from speech recognition in noise test, page 105

\section{Glossary}





\section{Chapter 1}

\section{General Provisions}

\section{1-1. Purpose}

This regulation governs-

a. Medical fitness standards for enlistment, induction, and appointment, including officer procurement programs.

$b$. Medical fitness standards for retention and separation, including retirement.

c. Medical fitness standards for diving, Special Forces, Airborne, Ranger, free fall parachute training and duty, and certain enlisted military occupational specialties (MOSs) and officer assignments.

$d$. Medical standards and policies for aviation.

$e$. Physical profiles.

$f$. Medical examinations and periodic health assessments.

\section{1-2. References}

Required and related publications and prescribed and referenced forms are listed in appendix A.

\section{1-3. Explanation of abbreviations and terms}

Abbreviations and special terms used in this regulation are explained in the glossary.

\section{1-4. Responsibilities}

a. The Surgeon General (TSG) will develop, revise, interpret, and disseminate current Army medical fitness standards and ensure Army compliance with Department of Defense (DOD) directives pertaining to those standards. TSG has the authority to issue exceptions to policies that are contained in this regulation.

$b$. Director, Department of Defense Medical Examination Review Board (DODMERB); Director, Army National Guard; Chief, U.S. Army Reserve (USAR); Superintendent, U.S. Military Academy (USMA), Director, Uniformed Services University of the Health Sciences (USUHS), and commanders of the U.S. Military Entrance Processing Command (MEPCOM), U.S. Army Recruiting Command (USAREC), U.S. Training and Doctrine Command, U.S. Army Medical Command (USAMEDCOM), U.S. Army Human Resources Command (AHRC), State Adjutants General, and all Army military treatment facilities (MTFs) worldwide, will implement policies prescribed in this regulation applicable to all Active Army and Reserve Component (RC) personnel and applicants for appointment (including all officer procurement programs), enlistment, and induction.

c. Commanders and military personnel officers at all levels of command will implement administrative and command provisions of chapters $5,7,8,9,10$, and 11 .

\section{1-5. Medical classification}

Individuals evaluated under the medical fitness standards contained in this regulation will be reported as indicated below.

a. Medically acceptable. Medical examiners will report as "medically acceptable" all individuals who meet the medical fitness standards established for the particular purpose for which examined. No individual will be accepted on a provisional basis subject to the successful treatment or correction of a disqualifying defect.

b. Medically unacceptable.

(1) Medical examiners will report as "medically unacceptable" by reason of medical unfitness all individuals who possess any one or more of the medical conditions or physical defects listed in this regulation as a cause for rejection for the specific purpose for which examined, except as noted in (2), below.

(2) Medical examiners will report as "Medically unacceptable-prior administrative waiver granted" all individuals who do not meet the medical fitness standards established for the particular purpose for which examined when a waiver has been previously granted and the applicable provisions of paragraph 1-6 apply.

\section{1-6. Review authorities and waivers}

a. Medical fitness standards cannot be waived by medical examiners or by the examinee.

$b$. Examinees initially reported as medically unacceptable by reason of medical unfitness when the medical fitness standards in chapter $2,3,4$, or 5 apply, may request a waiver of the medical fitness standards in accordance with the basic administrative directive governing the personnel action. Upon such request, the designated administrative authority or his or her designees for the purpose may grant such a waiver in accordance with current directives. The Office of the Surgeon General provides guidance when necessary to the review and waiver authorities on the interpretation of the medical standards and appropriateness of medical waivers. The Secretary of the Army is the waiver authority for accession. That authority is delegated down through the Deputy Chief of Staff, G-1 to the authorities listed in paragraphs $c$ through $i$, below.

c. The DODMERB, U.S. Air Force Academy, Colorado Springs, CO 80840-6518 is the sole review authority for reports of examinations given applicants for contracting into the Reserve Officers' Training Corps (ROTC) Programs. 
(See AR 40-29/NAVMEDCOMINST 6120.2/AFR 160-13/CG COMDTINST M6120.8.) Military Entrance Processing Stations (MEPS) under the purview of MEPCOM, are the review authorities for non-scholarship ROTC program examinations accomplished in their facilities. However, non-scholarship applicants who desire to contract into the ROTC program must have a qualifying DODMERB Review.

(1) The waiver authority for entry into ROTC programs is the Commanding General, ROTC Command.

(2) The waiver authority for applicants to USMA is the Superintendent, USMA. The waiver authority for commissioning USMA cadets is the DMPM, DCS, G-1, following consultation with TSG and receipt of a favorable waiver recommendation from the Superintendent, USMA.

d. Military Entrance Processing Stations (MEPS), under the purview of MEPCOM, are the review authorities for original enlistment examinations accomplished in their facilities. The Commanding General, USAREC, is the waiver authority for original enlistment. The Director, Army National Guard is the waiver authority for the Army National Guard (ARNG) and the Army National Guard of the United States (ARNGUS).

$e$. The waiver authority for direct accessions to Officer Candidate School (OCS) is the CG, USAREC. The waiver authority for medical waivers for ROTC, including Green to Gold candidates to OCS, is the CG, USACC. The waiver authority for currently serving enlisted Soldiers ("In-service") applicants to OCS is DCS, G-1. The application for a medical waiver to OCS will include a medical recommendation on the medical waiver from a military physician or military treatment facility (MTF) physician regarding any potential physical limitations or medical conditions and their possible impact upon an OCS applicant's ability to function as a military officer.

$f$. Review and waiver authority for other direct appointment programs (for example, Chaplain Corps) is USAREC. The waiver authority for initial selection for the Judge Advocate General Corps is AHRC.

g. Waiver authority for Special Forces training, Special Forces Assessment and Selection (SFAS), survival, evasion, resistance, escape (SERE) training, Military Freefall (MFF), and Special Forces Combat Diving Qualification Course (CDQC) is the Commandant, U.S. Army John F. Kennedy Special Warfare Center and School (USAJFKSWCS). Waiver authority for the Airborne School is the Commandant, U.S. Army Infantry School in coordination with AHRC.

$h$. Waivers for initial enlistment or appointment, including entrance and retention in officer procurement programs, will not be granted if the applicant does not meet the retention standards of chapter 3 . Requests from waiver authorities for exception to this policy will only be made under extraordinary circumstances and only with the approval of TSG (Headquarters, Department of the Army, (HQDA) (DASG-HS-AS)).

$i$. Waivers of medical fitness standards that have been previously granted apply automatically to subsequent medical actions pertinent to the program or purpose for which granted without the necessity of confirmation or termination when-

(1) The duration of the waiver was not limited at the time it was granted and the medical condition or physical defect has not interfered with the individual's successful performance of military duty.

(2) The medical condition or physical defect waived was below retention medical fitness standards applicable to the particular program involved and the medical condition or physical defect has remained essentially unchanged.

(3) The medical condition or physical defect waived was below procurement medical fitness standards applicable to the particular program involved and the medical condition or physical defect, although worse, is within the retention medical fitness standards prescribed for the program or purpose involved.

$k$. Exception to accession waiver for hearing: For waivers of hearing standards that are determined upon further testing to be worse than initially evaluated and will interfere with the individual's successful performance of military duty, the Soldier may be separated from military Service within the first 180 days for an existing prior to Service (EPTS) medical condition, provided an audiologist, entrance physical standards board (EPSBD), or medical board determines that no Service related cause or aggravation made the hearing worse than when initially evaluated.

\section{Chapter 2 \\ Physical Standards for Enlistment, Appointment, and Induction}

\section{2-1. General}

This chapter implements DODD 6130.3, Physical Standards for Appointment, Enlistment, and Induction, December 15, 2000, and DODI 6130.4, Medical Standards for Appointment, Enlistment, or Induction in the Armed Forces, January $18,2005$.

\section{2-2. Application and responsibilities}

a. Purpose. The purpose of the standards contained in this chapter is to ensure that individuals medically qualified are-

(1) Free of contagious diseases that would likely endanger the health of other personnel.

(2) Free of medical conditions or physical defects that would require excessive time lost from duty for necessary treatment or hospitalization or would likely result in separation from the Army for medical unfitness. 
(3) Medically capable of satisfactorily completing required training.

(4) Medically adaptable to the military environment without the necessity of geographical area limitations.

(5) Medically capable of performing duties without aggravation of existing physical defects or medical conditions.

$b$. Application. This chapter prescribes the medical conditions and physical defects that are causes for rejection for appointment, enlistment, and induction into military Service. Unless otherwise stipulated, the conditions listed in this chapter are those that would be disqualifying by virtue of current diagnosis, or for which the candidate has a verified past medical history. Other standards may be prescribed by DOD in the event of mobilization or a national emergency. Those individuals found medically qualified based on the medical standards of chapter 2 that were in effect prior to this publication will not be disqualified solely on the basis of the new standards. The designated waiver authorities may grant waivers for selection or continuation in the programs described below, provided the individual meets the retention standards of chapter 3. However, the standard in paragraph 2-30a will not be waived regardless of whether chapter 2 or chapter 3 standards are applied.

c. Scope. The standards of chapter 2 apply to-

(1) Applicants for appointment as commissioned or warrant officers in the Active Army and RC, including appointment as a Soldier in the USAR or the ARNG/ARNGUS. This includes enlisted Soldier applicants for appointment as commissioned or warrant officers. (However, for officers of the ARNG/ARNGUS or USAR who apply for appointment in the Active Army, the standards of chap 3 are applicable.)

(2) Applicants for enlistment in the Active Army, including the Delayed Entry/Future Soldier Program (delayed entry program). For medical conditions or physical defects predating original enlistment, these standards are applicable for enlistees' first 6 months of active duty. (However, for enlisted Soldiers of the ARNG/ARNGUS or USAR who apply for enlistment in the Active Army or who re-enter active duty for training (ADT) under the "split-training" option, the standards of chapter 3 are applicable.)

(a) Enlisted Soldiers identified within the first 6 months of active duty with a condition that existed prior to service that does not meet the standards of chapter 2 may be separated (or receive a waiver to remain on active duty) following an evaluation by an Entrance Physical Standards Board, in accordance with AR 635-200, chapter 5, with the exception as noted in $(b)$, below.

(b) Enlisted Soldiers identified within the first 6 months of active duty with a condition that existed prior to service that does not meet the standards of chapter 2 or chapter 3 must be evaluated by a medical evaluation board (MEB). The Soldier will then be referred to a physical evaluation board (PEB) unless the Soldier waives their right to the PEB in accordance with AR 635-40.

(3) Applicants for enlistment in the RC and Federally recognized units or organizations of the ARNG/ARNGUS. For medical conditions or physical defects predating original enlistment, these standards are applicable during the enlistees' initial period of ADT.

(4) Applicants for reenlistment in the Active Army, RC, and ARNG/ARNGUS after a period of more than 6 months has elapsed since discharge.

(5) Applicants (civilian applicants or enlisted Soldier applicants) for the USMA, Scholarship or Advanced Course ROTC, USUHS, Health Professions Scholarship Program (HPSP), Officer Candidate School (OCS), Warrant Officer Candidate School, and all other Army special officer personnel procurement programs. (See chap 3 for retention of students in HPSP and USUHS programs.)

(6) Retention of cadets and midshipmen at the U.S. Armed Forces academies and students enrolled in ROTC. (However, the Commander, ROTC Cadet Command or the Superintendent, USMA has the authority to grant medical waivers for continuation in these programs, provided the cadet meets the retention standards of chap 3.)

(7) All individuals being inducted into the Army.

d. Responsibilities. The Secretary of the Army will-

(1) Revise Army policies to conform to the standards contained in DOD Directive 6130.3 and DOD Instruction 6130.4 .

(2) Ensure uniformity of application and implementation of DOD Instruction 6130.4.

(3) Have authority to grant a waiver of the standards in individual cases for applicable reasons and ensure uniformity of waiver determinations. Delegated waiver authorities are noted in chapter 1.

(4) Have authority to change Army-specific visual standards (particularly for officer-accession programs) and establish other standards for special programs. Notification of any proposed changes in standards will be provided to the ASD(HA) 60 days before their implementation.

(5) Ensure that accurate International Classification of Disease (ICD) Codes are assigned to all medical conditions resulting in a personnel action such as medical waiver or medical separation.

(6) Eliminate inconsistencies and inequities based on race, sex, or examination location in the application of the standards.

e. Medical conditions. The disqualifying medical conditions are listed in paragraphs $2-3$ through $2-32$, below. (The ICD codes are listed in parentheses following each standard in chap 2.) Unless otherwise stipulated, the conditions 
listed in paragraphs 2-3 through 2-32, below, are those that would be disqualifying by virtue of current diagnosis, or for which the candidate has a verified past medical history.

\section{2-3. Abdominal organs and gastrointestinal system}

a. Esophagus. Current or history of esophageal disease, including, but not limited to ulceration, varices, fistula, achalasia, or Gastro-Esophageal Reflux Disease (GERD) (530.81), or complications from GERD including stricture, or maintenance on acid suppression medication, or other dysmotility disorders; chronic, or recurrent esophagitis (530.1), does not meet the standard. Current or history of reactive airway disease associated with GERD does not meet the standard. Current or history of dysmotility disorders, chronic, or recurrent esophagitis (530) does not meet the standard. History of surgical correction for GERD within 6 months does not meet the standard. (P42 esophageal correction, P43 stomach correction and P45 intestinal correction.)

b. Stomach and duodenum.

(1) Current gastritis, chronic or severe (535), or non-ulcerative dyspepsia that requires maintenance medication does not meet the standard.

(2) Current ulcer of stomach or duodenum confirmed by x-ray or endoscopy (533) does not meet the standard.

(3) History of surgery for peptic ulceration or perforation does not meet the standard.

c. Small and large intestine.

(1) Current or history of inflammatory bowel disease, including, but not limited to unspecified (558.9), regional enteritis or Crohn's disease (555), ulcerative colitis (556), or ulcerative proctitis (556), does not meet the standard.

(2) Current or history of intestinal malabsorption syndromes, including, but not limited to post-surgical and idiopathic (579), does not meet the standard. Lactase deficiency does not meet the standard only if of sufficient severity to require frequent intervention, or to interfere with normal function.

(3) Current or history of gastrointestinal functional and motility disorders within the past 2 years, including, but not limited to pseudo-obstruction, megacolon, history of volvulus, or chronic constipation and/or diarrhea (787.91), regardless of cause, persisting or symptomatic in the past 2 years, does not meet the standard.

(4) Current or history of irritable bowel syndrome (564.1) of sufficient severity to require frequent intervention or to interfere with normal function does not meet the standard.

(5) History of bowel resection does not meet the standard.

(6) Current symptomatic diverticular disease of the intestine does not meet the standard.

d. Gastrointestinal bleeding. History of gastrointestinal bleeding (578), including positive occult blood (792.1) if the cause has not been corrected, does not meet the standard. Meckel's diverticulum (751.0), if surgically corrected greater than 6 months prior, is not disqualifying.

e. Hepatic-biliary tract.

(1) Current acute or chronic hepatitis, hepatitis carrier state (070), hepatitis in the preceding 6 months, or persistence of symptoms after 6 months, or objective evidence of impairment of liver function does not meet the standard.

(2) Current or history of cirrhosis (571), hepatic cysts (573.8), abscess (572.0), or sequelae of chronic liver disease (571.3) does not meet the standard.

(3) Current or history of symptomatic cholecystitis, acute or chronic, with or without cholelithiasis (574), postcholecystectomy syndrome, or other disorders of the gallbladder, and biliary system (576) do not meet the standard. Cholecystectomy is not disqualifying if performed greater than 6 months prior to examination and patient remains asymptomatic. Fiberoptic procedure to correct sphincter dysfunction or cholelithiasis if performed greater than 6 months prior to examination and patient remains asymptomatic may not be disqualifying.

(4) Current or history of pancreatitis, acute (577.0) or chronic (577.1), does not meet the standard.

(5) Current or history of metabolic liver disease, including, but not limited to hemochromatosis (275.0), Wilson's disease (275.1), or alpha-1 anti-trypsin deficiency (277.6), does not meet the standard.

(6) Current enlargement of the liver from any cause (789.1) does not meet the standard.

f. Anorectal.

(1) Current anal fissure or anal fistula (565) does not meet the standard.

(2) Current or history of anal or rectal polyp (569.0), prolapse (569.1), stricture (569.2), or fecal incontinence (787. 6) within the last 2 years does not meet the standard.

(3) Current hemorrhoid (internal or external), when large, symptomatic, or with a history of bleeding (455) within the last 60 days, does not meet the standard.

g. Spleen.

(1) Current splenomegaly (789.2) does not meet the standard.

(2) History of splenectomy (P41.5) does not meet the standard, except when resulting from trauma.

h. Abdominal wall.

(1) Current hernia, including, but not limited to uncorrected inguinal (550) and other abdominal wall hernias (553), do not meet the standard. 
(2) History of open or laparoscopic abdominal surgery during the preceding 6 months (P54) does not meet the standard.

i. Other. History of any gastrointestinal procedure for the control of obesity does not meet the standard. Artificial openings, including, but not limited to ostomy (V44), do not meet the standard.

\section{2-4. Blood and blood-forming tissue diseases}

a. Anemia. Current hereditary or acquired anemia, which has not been corrected with therapy before appointment or induction, does not meet the standard. For the purposes of this regulation, anemia is defined as hemoglobin of less than 13.5 for males and less than 12 for females. The following ICD-9 codes are used for diagnosed anemia: hereditary hemolytic anemia (282), sickle cell disease (282.6), acquired hemolytic anemia (283), aplastic anemia (284), or unspecified anemias (285).

b. Hemorrhagic disorders. Current or history of coagulation defects (286) to include, but not limited to von Willebrand's Disease (286.4), idiopathic thrombocytopenia (287), or Henoch-Schönlein Purpura (287.0), does not meet the standard.

c. Leukopenia. Current or history of diagnosis of any form of chronic or recurrent agranulocytosis and/or leukopenia (288.0) does not meet the standard.

\section{2-5. Dental}

a. Current diseases of the jaws or associated tissues that prevent normal functioning do not meet the standard. Those diseases include, but are not limited to temporomandibular disorders (524.6) and/or myofascial pain that have not been corrected.

$b$. Current severe malocclusion (524), which interferes with normal mastication or requires early and protracted treatment, or a relationship between the mandible and maxilla that prevents satisfactory future prosthodontic replacement does not meet the standard.

c. Current insufficient natural healthy teeth (521) or lack of a serviceable prosthesis that prevents adequate incision and mastication of a normal diet and/or includes complex (multiple fixtures) dental implant systems with associated complications do not meet the standard. Individuals undergoing endodontic care are acceptable for entry in the Delayed Entry Program only if a civilian or military provider provides documentation that active endodontic treatment will be completed prior to being sworn into active duty.

$d$. Current orthodontic appliances for continued treatment (V53.4) do not meet the standard. Retainer appliances are permissible, provided all active orthodontic treatment has been satisfactorily completed. Individuals undergoing orthodontic care are acceptable for enlistment in the Delayed Entry Program only if a civilian or military orthodontist provides documentation that active orthodontic treatment will be completed prior to being sworn into active duty.

\section{2-6. Ears}

a. External ear. Current atresia (744.02) or severe microtia (744.23), congenital or acquired stenosis (380.5), chronic otitis externa (380.2), or severe external ear deformity (744.3) that prevents or interferes with the proper wearing of hearing protection does not meet the standard.

b. Mastoids. Current or history of mastoiditis (383.9), residual with fistula (383.81), chronic drainage or conditions requiring frequent cleaning of the mastoid bone do not meet the standard. Marked external deformity that prevents or interferes with wearing a protective mask or helmet (383.3) does not meet the standard.

c. Ménière's syndrome. Current or history of Ménière's syndrome or other chronic diseases of the vestibular system (386) does not meet the standard.

d. Middle and inner ear. Current or history of chronic otitis media (382), cholesteatoma (385.3), or history of any inner (P20) or middle (P19) ear surgery (including cochlear implantation) does not meet the standard. Myringotomy or successful tympanoplasty is not disqualifying.

e. Tympanic membrane. Current perforation of the tympanic membrane (384.2) or history of surgery to correct perforation during the preceding 120 days (P19) does not meet the standard.

\section{2-7. Hearing}

a. Audiometers, calibrated to standards of the International Standards Organization (ISO 8253:1 1989) (reference (c)) or the American National Standards Institute (ANSI 1996), will be used to test the hearing of all applicants.

$b$. All audiometric tracings or audiometric readings recorded on reports of medical examination or other medical records will be clearly identified.

c. Current hearing threshold level in either ear greater than that described below does not meet the standard:

(1) Pure tone at 500, 1000, and 2000 cycles per second for each ear of not more than 30 decibels (dB) on the average, with no individual level greater than $35 \mathrm{~dB}$ at those frequencies.

(2) Pure tone level not more than $45 \mathrm{~dB}$ at 3000 cycles per second or $55 \mathrm{~dB}$ at 4000 cycles per second for each ear.

(3) There is no standard for 6000 cycles per second. 
d. Current or history of hearing aid use (V53.2) does not meet the standard.

\section{2-8. Endocrine and metabolic disorders}

a. Current or history of adrenal dysfunction (255) does not meet the standard.

$b$. Current or history of diabetes mellitus (250) does not meet the standard.

c. Current persistent glycosuria when associated with impaired glucose tolerance (250) or renal tubular defects (271.

4) does not meet the standard.

$d$. Current or history of acromegaly, including, but not limited to gigantism or other disorders of pituitary function (253), does not meet the standard.

$e$. Current or history of gout (274) does not meet the standard.

$f$. Current or history of hyperinsulinism (251.1) does not meet the standard.

g. Current or history of hyperparathyroidism (252.0) and hypoparathyroidism (252.1) does not meet the standard.

$h$. Thyroid disorders.

(1) Current goiter (240) does not meet the standard.

(2) Current hypothyroidism uncontrolled by medication (244) does not meet the standard.

(3) Current or history of hyperthyroidism (242.9) does not meet the standard.

(4) Current thyroiditis (245) does not meet the standard.

i. Current nutritional deficiency diseases, including, but not limited to beriberi (265), pellagra (265.2), and scurvy (267) do not meet the standard.

$j$. Other endocrine or metabolic disorders such as cystic fibrosis (277), porphyria (277.1), and amyloidosis (277.3) that obviously prevent satisfactory performance of duty or require frequent or prolonged treatment do not meet the standard.

\section{2-9. Upper extremities}

a. Limitation of motion. Current joint ranges of motion less than the measurements listed below do not meet the standard.

(1) Shoulder (726.1):

(a) Forward elevation to 90 degrees.

(b) Abduction to 90 degrees.

(2) Elbow (726.3):

(a) Flexion to 100 degrees.

(b) Extension to 15 degrees.

(3) Wrist (726.4): A total range of 60 degrees (extension plus flexion) or radial and ulnar deviation combined arc 30 degrees.

(4) Hand (726.4):

(a) Pronation to 45 degrees.

(b) Supination to 45 degrees.

(5) Fingers and thumb (726.4): Inability to clench fist, pick up a pin, grasp an object, or touch tips of at least three fingers with thumb.

b. Hand and fingers.

(1) Current absence of the distal phalanx of either thumb (885) does not meet the standard.

(2) Current absence of distal and middle phalanx of an index, middle, or ring finger of either hand, irrespective of the absence of the little finger (886), does not meet the standard.

(3) Current absence of more than the distal phalanx of any two of the following fingers: index, middle finger, or ring finger of either hand (886) does not meet the standard.

(4) Current absence of hand or any portion thereof (887) does not meet the standard except for specific absences of fingers as noted above.

(5) Current polydactyly (755) does not meet the standard.

(6) Scars and deformities of the fingers or hand (905.2) that are symptomatic or that impair normal function to such a degree as to interfere with the satisfactory performance of military duty do not meet the standard (see also para 2-32).

(7) Current intrinsic paralysis or weakness of upper limbs, including nerve paralysis, carpal tunnel and cubital syndromes, lesion of ulnar and radial nerve (354) sufficient to produce physical findings in the hand, such as muscle atrophy and weakness does not meet the standard.

(8) Current disease, injury, or congenital condition with residual weakness or symptoms such as to prevent satisfactory performance of duty, including, but not limited to chronic joint pain: shoulder (719.41), upper arm (719. 42), forearm (719.43), and hand (719.44), late effect of fracture of the upper extremities (905.2), late effect of sprains without mention of injury (905.7), and late effects of tendon injury (905.8) do not meet the standard. 
(See also para 2-11.)

\section{2-10. Lower extremities}

a. Limitation of motion. Current joint ranges of motion less than the measurements listed in paragraphs below do not meet the standard.

(1) Hip (due to disease (726.5), or injury (905.2)):

(a) Flexion to 90 degrees.

(b) No demonstrable flexion contracture.

(c) Extension to 10 degrees (beyond 0 degrees).

(d) Abduction to 45 degrees.

(e) Rotation of 60 degrees (internal and external combined).

(2) Knee (due to disease (726.6), or injury (905.4)):

(a) Full extension to 0 degrees.

(b) Flexion to 110 degrees.

(3) Ankle (due to disease (726.7), or injury (905.4) or congenital defect):

(a) Dorsiflexion to 10 degrees.

(b) Planter flexion to 30 degrees.

(4) Subtalar eversion and inversion totaling 5 degrees (due to disease (726.7) or injury (905.4) or congenital defect).

b. Foot and ankle.

(1) Current absence of a foot or any portion thereof (896) does not meet the standard.

(2) Current or history of deformities of the toes (acquired (735) or congenital (755.66)) including, but not limited to conditions such as hallux valgus (735.0), hallux varus (735.1), hallux rigidicus (735.2), hammer toe(s) (735.4), claw toe(s) (735.5), overriding toe(s) (735.8), that prevents the proper wearing of military footwear or impairs walking, marching, running, or jumping, do not meet the standard.

(3) Current or history of clubfoot (754.70) or pes cavus (754.71) that prevents the proper wearing of military footwear or impairs walking, marching, running, or jumping does not meet the standard.

(4) Current symptomatic pes planus (acquired (734) or congenital (754.6)) or history of pes planus corrected by prescription or custom orthotics does not meet the standard.

(5) Current ingrown toenails (703.0), if infected or symptomatic, do not meet the standard.

(6) Current plantar fasciitis (728.71) does not meet the standard.

(7) Current neuroma (355.6) that is refractory to medical treatment, or impairs walking, marching, running, or jumping, or prevents the proper wearing of military footwear, does not meet the standard.

c. Leg, knee, thigh, and hip.

(1) Current loose or foreign body within the knee joint (717.6) does not meet the standard.

(2) History of uncorrected anterior (717.83) or posterior (717.84) cruciate ligament injury does not meet the standard. History of surgical correction of knee ligaments does not meet the standard only if symptomatic or unstable (P81.4).

(3) Current symptomatic medial and lateral collateral ligament injury does not meet the standard.

(4) Current symptomatic medial and lateral meniscal injury does not meet the standard.

(5) Current unspecified internal derangement of the knee (717.9) does not meet the standard.

(6) Current or history of congenital dislocation of the hip (754.3), osteochondritis of the hip (Legg-Perthes disease) (732.1), or slipped femoral epiphysis of the hip (732.2) does not meet the standard.

(7) Current or history of hip dislocation (835) within 2 years preceding examination does not meet the standard.

(8) Current osteochondritis of the tibial tuberosity (Osgood-Schlatter disease) (732.4), does not meet the standard if symptomatic.

d. General.

(1) Current deformities, disease, or chronic joint pain of pelvic region, thigh (719.45), lower leg (719.46), ankle and/ or foot (719.47) that have interfered with function to such a degree as to prevent the individual from following a physically active vocation in civilian life, or that would interfere with walking, running, weight bearing, or the satisfactory completion of training or military duty, do not meet the standard.

(2) Current leg-length discrepancy resulting in a limp (736.81) does not meet the standard.

(See also para 2-11.)

\section{2-11. Miscellaneous conditions of the extremities}

a. Current or history of chondromalacia (717.7), including, but not limited to chronic patello-femoral pain syndrome and retro-patellar pain syndrome, chronic osteoarthritis (715.3) or traumatic arthritis (716.1) does not meet the standard.

$b$. Current joint dislocation if unreduced, or history of recurrent dislocations of any major joint such as shoulder (831), hip (835), elbow (832), knee (836), ankle (837), or instability of any major joint (shoulder (718.81), elbow (718. 
82), hip (718.85), ankle and foot (718.87) or multiple sites (718.89)) does not meet the standard. History of recurrent instability of the knee or shoulder does not meet the standard.

c. Current or history of chronic osteoarthritis (715.3) or traumatic arthritis (716.1) of isolated joints of more than a minimal degree that has interfered with the following of a physically active vocation in civilian life, or that prevents the satisfactory performance of military duty does not meet the standard.

d. Fractures.

(1) Current malunion or non-union of any fracture (733.8) (except asymptomatic ulnar styloid process fracture) does not meet the standard.

(2) Current retained hardware that is symptomatic, interferes with proper wearing of protective equipment or military uniform, and/or is subject to easy trauma, does not meet the standard (V53.7). Retained hardware (733.99) (including plates, pins, rods, wires, or screws used for fixation) is not disqualifying if fractures are healed, ligaments are stable, there is no pain, and it is not subject to easy trauma.

$e$. Current devices, including, but not limited to silastic or titanium, implanted to correct orthopedic abnormalities (V43), do not meet the standard.

$f$. Current or history of contusion of bone or joint; an injury of more than a minor nature that will interfere or prevent performance of military duty, or will require frequent or prolonged treatment without fracture nerve injury, open wound, crush or dislocation, which occurred within the preceding 6 weeks (upper extremity (923), lower extremity (924), ribs and clavicle (922)) does not meet the standard.

g. History of joint replacement (V43.6) of any site does not meet the standard.

h. Current or history of muscular paralysis, contracture, or atrophy (728), if progressive or of sufficient degree to interfere with or prevent satisfactory performance of military duty or if it will require frequent or prolonged treatment, does not meet the standard.

i. Current osteochondritis dessicans (732.7) does not meet the standard.

$j$. Current or history of osteochondromatosis or multiple cartilaginous exostoses (727.82) do not meet the standard.

$k$. Current osteoporosis (733) does not meet the standard.

l. Current osteomyelitis (730), or history of recurrent osteomyelitis does not meet the standard.

(See also paras 2-9 and 2-10.)

\section{2-12. Eyes}

a. Lids.

(1) Current blepharitis (373), chronic or acute, until cured (373.00), does not meet the standard.

(2) Current blepharospasm (333.81) does not meet the standard.

(3) Current dacryocystitis, acute or chronic (375.30) does not meet the standard.

(4) Deformity of the lids (374.4), complete or extensive lid deformity, sufficient to interfere with vision or impair protection of the eye from exposure does not meet the standard.

(5) Current growths or tumors of the eyelid, other than small, non-progressive, asymptomatic, benign lesions, do not meet the standard.

b. Conjunctiva.

(1) Current chronic conjunctivitis (372.1), including, but not limited to trachoma (076) and chronic allergic conjunctivitis (372.14), does not meet the standard.

(2) Current or recurrent pterygium, (372.4), if condition encroaches on the cornea in excess of $3 \mathrm{~mm}$, or interferes with vision, or is a progressive peripheral pterygium (372.42), or recurring pterygium after two operative procedures (372.45), does not meet the standard.

(3) Current xerophthalmia (372.53) does not meet the standard.

c. Cornea.

(1) Current or history of corneal dystrophy of any type (371.5), including but not limited to keratoconus (371.6) of any degree does not meet the standard.

(2) History of refractive surgery including, but not limited to: Lamellar (P11.7) and/or penetrating keratoplasty (P11. 6). Radial Keratotomy and Astigmatic Keratotomy does not meet the standard. Refractive surgery performed with an Excimer Laser, including but not limited to, Photorefractive Keratectomy (commonly known as PRK), Laser Epithelial Keratomileusis (commonly known as LASEK), and Laser-Assisted in situ Keratomileusis (commonly known as LASIK) (P11.7) does not meet the standard if any of the following conditions are met:

(a) Pre-surgical refractive error in either eye exceeds +8.00 to -8.00 diopters.

(b) At least 6 months recovery period has not occurred between last refractive surgery or augmenting procedure and accession medical examination.

(c) There have been complications, and/or medications or ophthalmic solutions are required.

(d) Post-surgical refraction in each eye is not stable as demonstrated by-

1. At least two separate refractions at least one month apart, the most recent of which demonstrates more than $+/-0$. 50 diopters difference for spherical vision and/or more than +/- 0.25 diopters for cylinder vision; and 
2. At least 3 months recovery has not occurred between the last refractive surgery or augmenting procedure and one of the comparison refractions.

(e) Pre-surgical and post-surgical refractive error does not meet the standards for the military Service to which the candidate is applying.

(3) Current keratitis (370), acute or chronic, including, but not limited to recurrent corneal ulcers (370.0), erosions (abrasions), or herpetic ulcers (054.42) does not meet the standard.

(4) Current corneal vascularization (370.6) or corneal opacification (371) from any cause that is progressive or reduces vision below the standards prescribed in paragraph 2-13 does not meet the standard.

d. Uveitis or iridocyclitis. Current or history of uveitis or iridocyclitis (364.3) does not meet the standard.

e. Retina.

(1) Current or history of retinal defects and dystrophies, angiomatoses (759.6), retinoschisis and retinal cysts (361. 1), phakomas (362.89), and other congenito-retinal hereditary conditions (362.7) that impair visual function or are progressive, do not meet the standard.

(2) Current or history of any chorioretinal or retinal inflammatory conditions, including, but not limited to conditions leading to neovascularization, chorioretinitis, histoplasmosis, toxoplasmosis, or vascular conditions of the eye to include Coats' disease, or Eales' disease (363) does not meet the standard.

(3) Current or history of degenerative changes of any part of the retina (362) does not meet the standard.

(4) Current or history of detachment of the retina (361), history of surgery for same, or peripheral retinal injury, defect (361.3), or degeneration that may cause retinal detachment does not meet the standard.

f. Optic nerve.

(1) Current or history of optic neuritis (377.3), including, but not limited to neuroretinitis, secondary optic atrophy, or documented history of retrobulbar neuritis does not meet the standard.

(2) Current or history of optic atrophy (377.1), or cortical blindness (377.75) does not meet the standard.

(3) Current or history of papilledema (377.0) does not meet the standard.

g. Lens.

(1) Current aphakia (379.31), history of lens implant, or current or history of dislocation of a lens does not meet the standard.

(2) Current or history of opacities of the lens (366) that interfere with vision or that are considered to be progressive, including cataract (366.9), do not meet the standard.

h. Ocular mobility and motility.

(1) Current diplopia (386.2) does not meet the standard.

(2) Current nystagmus (379.50) other than physiologic "end-point nystagmus" does not meet the standard.

(3) Esotropia (378.0), and hypertropia (378.31). For entrance into the USMA or ROTC programs, the following conditions are also disqualifying: esotropia of over 15 prism diopters; exotropia of over 10 prism diopters; hypertropia of over 5 prism diopters.

i. Miscellaneous defects and conditions.

(1) Current or history of abnormal visual fields due to disease of the eye or central nervous system (368.4), or trauma (368.9) does not meet the standard.

(2) Absence of an eye, clinical anophthalmos, unspecified congenital (743.00) or acquired, or current or history of other disorders of globe (360.8) does not meet the standard.

(3) Current asthenopia (368.13), does not meet the standard.

(4) Current unilateral or bilateral non-familial exophthalmos (376) does not meet the standard.

(5) Current or history of glaucoma (365), including, but not limited to primary, secondary, or pre-glaucoma as evidenced by intraocular pressure above 21 millimeters of mercury $(\mathrm{mmHg}$ ), or changes in the optic disc or visual field loss associated with glaucoma, does not meet the standard.

(6) Current loss of normal pupillary reflex reactions to accommodation (367.5) or light (379.4), including Adie's syndrome, does not meet the standard.

(7) Current night blindness (368.6) does not meet the standard.

(8) Current or history of retained intraocular foreign body (360) does not meet the standard.

(9) Current or history of any organic disease of the eye (360) or adnexa (376) not specified above, which threatens vision or visual function, does not meet the standard.

\section{2-13. Vision}

$a$. Current distant visual acuity of any degree that does not correct with spectacle lenses to at least one of the following (367) does not meet the standard:

(1) $20 / 40$ in one eye and $20 / 70$ in the other eye.

(2) $20 / 30$ in one eye and $20 / 100$ in the other eye.

(3) $20 / 20$ in one eye and $20 / 400$ in the other eye. 
$b$. However, for entrance into USMA or ROTC, distant visual acuity that does not correct to 20/20 in one eye and $20 / 40$ in the other eye does not meet the standard. For entrance into OCS, distant visual acuity that does not correct to $20 / 20$ in one eye and 20/100 in the other eye does not meet the standard.

c. Current near visual acuity (367) of any degree that does not correct to 20/40 in the better eye does not meet the standard.

d. Current refractive error (hyperopia (367.0), myopia (367.1), astigmatism (367.2)), or history of refractive error prior to any refractive surgery manifest by any refractive error in spherical equivalent of worse than -8.00 or +8.00 diopters does not meet the standard. However, for entrance into USMA or Army ROTC programs, the following conditions do not meet the standard:

(1) Astigmatism, all types over 3 diopters.

(2) Hyperopia over 8.00 diopters spherical equivalent.

(3) Myopia over 8 diopters spherical equivalent.

(4) Refractive error corrected by orthokeratology or keratorefractive surgery.

e. Contact lenses. Current complicated cases requiring contact lenses for adequate correction of vision, such as corneal scars (371) and irregular astigmatism (367.2), do not meet the standard.

$f$. Color vision (368.5). Failure to pass a color vision test is not an automatic disqualification. Although there is no standard, color vision will be tested because adequate color vision is a prerequisite for entry into many military specialties. However, for entrance into the USMA or Army ROTC or OCS programs, the inability to distinguish and identify without confusion the color of an object, substance, material, or light that is uniformly colored a vivid red or vivid green does not meet the standard.

\section{2-14. Genitalia}

a. Female genitalia.

(1) Current or history of abnormal uterine bleeding (626.2), including, but not limited to menorrhagia, metrorrhagia, or polymenorrhea, does not meet the standard.

(2) Current unexplained amenorrhea (626.0) does not meet the standard.

(3) Current or history of dysmenorrhea (625.3) that is incapacitating to a degree recurrently necessitating absences of more than a few hours from routine activities does not meet the standard.

(4) Current or history of endometriosis (617) does not meet the standard.

(5) History of major abnormalities or defects of the genitalia such as change of sex (P64.5), hermaphroditism, pseudohermaphroditism, or pure gonadal dysgenesis (752.7), or dysfunctional residuals from surgical correction of these conditions does not meet the standard.

(6) Current or history of ovarian cysts (620.2), when persistent or symptomatic does not meet the standard.

(7) Current pelvic inflammatory disease (614), or history of recurrent pelvic inflammatory disease, does not meet the standard. Current or history of chronic pelvic pain or unspecified symptoms associated with female genital organs (625. 9) does not meet the standard.

(8) Current pregnancy (V22) does not meet the standard until 6 months after the end of the pregnancy.

(9) Uterus, congenital absence of (752.3), or enlargement due to any cause (621.2) does not meet the standard.

(10) Current or history of genital infection or ulceration, including but not limited to herpes genitalis (054.11) or condyloma acuminatum (078.11), if of sufficient severity to require frequent intervention or to interfere with normal function, does not meet the standard.

(11) Current abnormal gynecologic cytology, including, but not limited to unspecified abnormalities of the Papanicolaou smear of the cervix (Pap smear) (795) excluding Human Papilloma Virus (HPV) (079.4) or confirmed Low-Grade Squamous Intraepithelial Lesion (LGSIL) (622.9), does not meet the standard. For the purposes of this regulation, confirmation is by colposcopy or repeat cytology.

b. Male genitalia.

(1) Current absence of one or both testicles, either congenital (752.89) or undescended (752.51) does not meet the standard.

(2) Current epispadias (752.62) or hypospadias (752.61), when accompanied by evidence of urinary tract infection, urethral stricture, or voiding dysfunction, does not meet the standard.

(3) Current enlargement or mass of testicle or epididymis (608.9) does not meet the standard.

(4) Current orchitis (604) or epididymitis (604.90) does not meet the standard.

(5) History of penis amputation (878.0) does not meet the standard.

(6) Current or history of genital infection or ulceration, including, but not limited to herpes genitalis (054.13) and condyloma acuminatum (078.11), if of sufficient severity to require frequent intervention or to interfere with normal function, does not meet the standard

(7) Current acute prostatitis (601.0) or chronic prostatitis (601.1) does not meet the standard.

(8) Current hydrocele (603.0), if large or symptomatic, does not meet the standard. Left varicocele (456.4), if 
symptomatic, or associated with testicular atrophy, or vericocele larger than the testis does not meet the standard. Any right varicocele (456.4) does not meet the standard.

c. Current or history of chronic scrotal pain or unspecified symptoms associated with male genital organs (608.9) do not meet the standard.

$d$. History of major abnormalities or defects of the genitalia, such as a change of sex (P64.5), hermaphroditism, pseudohermaphroditism, or pure gonadal dysgenesis (752.7) or dysfunctional residuals from surgical correction of these conditions does not meet the standard.

\section{2-15. Urinary system}

a. Current cystitis (595), or history of chronic or recurrent cystitis does not meet the standard.

$b$. Current urethritis (597.80), or history of chronic or recurrent urethritis does not meet the standard.

c. History of enuresis (788.30) or incontinence of urine after 13th birthday does not meet the standard (see also para 2-29).

$d$. Current hematuria (599.7), pyuria, or other findings indicative of urinary tract disease (599) does not meet the standard.

e. Current urethral stricture (598) or fistula (599.1) does not meet the standard.

$f$. Kidney.

(1) Current absence of one kidney, congenital (753.0) or acquired (V45.73) does not meet the standard.

(2) Current pyelonephritis (chronic or recurrent) (590.0) or any other unspecified infections of the kidney (590.9) does not meet the standard.

(3) Current or history of polycystic kidney (753.1) does not meet the standard.

(4) Current or history of horseshoe kidney (753.3) does not meet the standard.

(5) Current or history of hydronephrosis (591) does not meet the standard.

(6) Current or history of acute (580) or chronic (582) nephritis of any type does not meet the standard.

g. Current or history of proteinuria (791.0) (greater than 200 milligrams (mg)/24 hours; or a protein to creatinine ratio greater than 0.2 in a random urine sample, if greater than 48 hours after strenuous activity) does not meet the standard, unless consultation determines the condition to be benign orthostatic proteinuria.

$h$. Current or history of urolithiasis (592) within the preceding 12 months does not meet the standard. Recurrent calculus, nephrocalcinosis, or bilateral renal calculi at any time, does not meet the standard.

\section{2-16. Head}

a. Uncorrected deformities of the skull, face, or mandible (754.0) of a degree that would prevent the individual from wearing a protective mask or military headgear do not meet the standard.

$b$. Loss or absence of the bony substance of the skull (756.0 or 738.1) not successfully corrected by reconstructive materials, or leaving residual defect in excess of 1 square inch $(6.45$ centimeter $(\mathrm{cm}) 2)$ or the size of a 25 cent piece does not meet the standard.

\section{2-17. Neck}

a. Current symptomatic cervical ribs (756.2) do not meet the standard.

$b$. Current or history of congenital cysts (744.4) of branchial cleft origin or those developing from remnants of the thyroglossal duct, with or without fistulous tracts, does not meet the standard.

c. Current contraction (723) of the muscles of the neck, spastic or non-spastic, or cicatricial contracture of the neck, to the extent that it interferes with the proper wearing of a uniform or military equipment or is so disfiguring as to interfere with or prevent satisfactory performance of military duty, does not meet the standard.

\section{2-18. Heart}

a. Current or history of all valvular heart diseases, congenital (746) or acquired (394), including those improved by surgery, do not meet the standard. Mitral valve prolapse or bicuspid aortic valve is not disqualifying unless there is associated tachyarrhythmia, mitral regurgitation, aortic stenosis, insufficiency, or cardiomegaly.

$b$. Current or history coronary heart disease (410) does not meet the standard.

c. Current or history of symptomatic arrhythmia or electrocardiographic evidence of arrhythmia.

(1) Current or history of supraventricular tachycardia (427.0), or any arrhythmia originating from the atrium or sinoatrial node, such as atrial flutter, and atrial fibrillation, unless there has been no recurrence during the preceding 2 years while off all medications, does not meet the standard. Premature atrial or ventricular contractions sufficiently symptomatic to require treatment, or result in physical or psychological impairment, do not meet the standard.

(2) Current or history of ventricular arrhythmias (427.1), including ventricular fibrillation, tachycardia, or multifocal premature ventricular contractions, does not meet the standard. Occasional asymptomatic unifocal premature ventricular contractions are not disqualifying.

(3) Current or history of ventricular conduction disorders, including, but not limited to disorders with left bundle 
branch block (426.2), Mobitz type II second degree atrioventricular (AV) block (426.12), and third degree AV block (426.0), and Lown-Ganong-Levine-Syndrome (426.81) associated with an arrhythmia do not meet the standard. Current or history of Wolff-Parkinson-White Syndrome (426.7), unless it has been successfully ablated for a period of 2 years without recurrence of arrhythmia and now with a normal electrocardiogram, does not meet the standard.

(4) Current or history of conduction disturbances such as first degree AV block (426.11), left anterior hemiblock (426.2), right bundle branch block (426.4), or Mobitz type I second degree AV block (426.13) do not meet the standard when symptomatic or associated with underlying cardiovascular disease.

d. Current cardiomegaly, hypertrophy, or dilatation of the heart (429.3) do not meet the standard.

e. Current or history of cardiomyopathy (425), including myocarditis (422), or congestive heart failure (428), does not meet the standard.

f. Current or history of pericarditis (420) (acute nonrheumatic), unless the individual is free of all symptoms for 2 years, and has no evidence of cardiac restriction or persistent pericardial effusion, does not meet the standard.

g. Current persistent tachycardia (785.1) (resting pulse rate of 100 beats per minute or greater) does not meet the standard.

h. Current or history of congenital anomalies of heart and great vessels (746), except for corrected patent ductus arteriosus, do not meet the standard.

\section{2-19. Vascular system}

a. Current or history of abnormalities of the arteries and blood vessels (447), including, but not limited to aneurysms (442), atherosclerosis (440), or arteritis (446), do not meet the standard.

$b$. Current or history of hypertensive vascular disease (401) does not meet the standard. Elevated blood pressure defined as the average of three consecutive sitting blood pressure measurements separated by at least 10 minutes, diastolic greater than $90 \mathrm{mmHg}$ or three consecutive systolic pressure measurements greater than $140 \mathrm{mmHg}$ does not meet the standard (796.2).

c. History of pulmonary (415) or systemic embolization (444) does not meet the standard.

$d$. Current or history of peripheral vascular disease (443), including, but not limited to diseases such as Raynaud's Disease (443.0) does not meet the standard.

$e$. Current or history of venous diseases, including but not limited to, recurrent thrombophlebitis (451), thrombophlebitis during the preceding year, or any evidence of venous incompetence, such as large or symptomatic varicose veins, edema, or skin ulceration (454), does not meet the standard.

\section{2-20. Height}

The causes for disqualification are-

a. Men: Height below 60 inches or over 80 inches does not meet the standard.

$b$. Women: Height below 58 inches or over 80 inches does not meet the standard.

\section{2-21. Weight}

a. Army applicants for initial appointment as commissioned officers (to include appointment as commissioned warrant officers) must meet the standards of AR 600-9. Body fat composition is used as the final determinant in evaluating an applicant's acceptability when the weight exceeds that listed in the weight tables.

b. All other applicants must meet the standards of tables 2-1 and 2-2. Body fat composition is used as the final determinant in evaluating an applicant's acceptability when the weight exceeds that listed in the weight tables.

\section{2-22. Body build}

The cause for rejection for appointment, enlistment, and induction is deficient muscular development that would interfere with the completion of required training.

\section{2-23. Lungs, chest wall, pleura, and mediastinum}

a. Current abnormal elevation of the diaphragm, either side, does not meet the standard. Any nonspecific abnormal findings on radiological and other examination of body structure, such as lung field (793.1), or other thoracic or abdominal organ (793.2), does not meet the standard.

$b$. Current abscess of the lung or mediastinum (513) does not meet the standard.

c. Current or history of acute infectious processes of the lung, including but not limited to viral pneumonia (480), pneumococcal pneumonia (481), bacterial pneumonia (482), pneumonia other specified (483), pneumonia infectious disease classified elsewhere (484), bronchopneumonia organism unspecified (485), pneumonia organism unspecified (486), do not meet the standard until cured.

d. Asthma (493), including reactive airway disease, exercise-induced bronchospasm or asthmatic bronchitis, reliably diagnosed and symptomatic after the 13th birthday, does not meet the standard. Reliable diagnostic criteria may include any of the following elements: substantiated history of cough, wheeze, chest tightness, and/or dyspnea that persists or recurs over a prolonged period of time, generally more than 12 months. 
e. Current bronchitis (490), acute or chronic, symptoms over 3 months occurring at least twice a year (491), does not meet the standard.

$f$. Current or history of bronchiectasis (494) does not meet the standard.

g. Current or history of bronchopleural fistula (510) unless resolved with no sequelae does not meet the standard.

$h$. Current or history of bullous or generalized pulmonary emphysema (492) does not meet the standard.

$i$. Current chest wall malformation (754), including, but not limited to pectus excavatum (754.81), or pectus carinatum (754.82), if these conditions interfere with vigorous physical exertion, does not meet the standard.

$j$. History of empyema (510) does not meet the standard.

$k$. Current pulmonary fibrosis (515) from any cause producing respiratory symptoms does not meet the standard.

l. Current foreign body in lung, trachea, or bronchus (934) does not meet the standard.

$m$. History of lobectomy (P32.4) does not meet the standard.

$n$. Current or history of pleurisy with effusion (511.9) within the previous 2 years does not meet the standard.

$o$. Current or history of pneumothorax (512) occurring during the year preceding examination, if due to trauma or surgery or occurring during the 3 years preceding examination from spontaneous origin, does not meet the standard Recurrent spontaneous pneumothorax (512) does not meet the standard.

p. History of open or laparoscopic thoracic or chest wall (including breasts) surgery during the preceding 6 months (P54) does not meet the standard.

\section{2-24. Mouth}

a. Current cleft lip or palate defects (749), not satisfactorily repaired by surgery do not meet the standard.

$b$. Current leukoplakia (528.6) does not meet the standard.

\section{2-25. Nose, sinuses, and larynx}

a. Rhinitis.

(1) Current allergic rhinitis (477.0), due to pollen (477.8), or due to other allergen, or cause unspecified (477.9), if not controlled by oral medication or topical corticosteroid medication, does not meet the standard. History of allergic rhinitis immunotherapy within the previous year does not meet the standard.

(2) Current chronic non-allergic rhinitis (472.0), if not controlled by oral medication or topical corticosteroid medication, does not meet the standard.

$b$. Current chronic conditions of larynx including vocal cord paralysis (478.3), chronic hoarseness, chronic laryngitis, larynx ulceration, polyps, granulation tissue, or other symptomatic disease of larynx, vocal cord dysfunction not elsewhere classified (478.7) do not meet the standard.

c. Current anosmia or parosmia (781.1) does not meet the standard.

d. History of recurrent epistaxis (784.7), with greater than one episode per week of bright red blood from the nose occurring over a 3-month period, does not meet the standard.

e. Current nasal polyps (471) or history of nasal polyps, unless greater than 12 months has elapsed since nasal polypectomy, does not meet the standard.

$f$. Current perforation of nasal septum (478.1) does not meet the standard.

g. Current chronic sinusitis (473), or current acute sinusitis (461.9), does not meet the standard. Such conditions exist when evidenced by chronic purulent nasal discharge, hyperplastic changes of the nasal tissue, symptoms requiring frequent medical attention, or x-ray findings.

h. Current or history of tracheostomy (V44.0) or tracheal fistula (530.84) does not meet the standard.

$i$. Current or history of deformities, or conditions or anomalies (750.9) of the upper alimentary tract, of the mouth, tongue, palate throat, pharynx, larynx, and nose that interfere with chewing, swallowing, speech, or breathing do not meet the standard.

j. Current chronic pharyngitis (462) and chronic nasopharyngitis (472.2), do not meet the standard.

\section{2-26. Neurological disorders}

a. Current or history of cerebrovascular conditions, including but not limited to subarachnoid (430) or intracerebral (431) hemorrhage, vascular insufficiency, aneurysm, or arteriovenous malformation (437), do not meet the standard.

$b$. History of congenital or acquired anomalies of the central nervous system (742), or meningocele (741.9), does not meet the standard.

c. Current or history of disorders of meninges, including, but not limited to cysts (349.2), does not meet the standard.

$d$. Current or history of degenerative and hereditodegenerative disorders, including, but not limited to those disorders affecting the cerebrum (330), basal ganglia (333), cerebellum (334), spinal cord (335), or peripheral nerves (337), do not meet the standard.

$e$. History of recurrent headaches (784.0), including, but not limited to, migraines (346) and tension headaches (307. 
81) that interfere with normal function in the past 3 years, or of such severity to require prescription medications, do not meet the standard.

$f$. Head injury (854.0).

(1) History of head injury will be disqualifying if associated with any of the following:

(a) Post-traumatic seizure(s) occurring more than 30 minutes after injury.

(b) Persistent motor or sensory deficits.

(c) Impairment of intellectual function.

(d) Alteration of personality.

(e) Unconsciousness, amnesia, or disorientation of person, place, or time of 24-hours duration or longer post-injury.

(f) Multiple fractures involving skull or face (804).

(g) Cerebral laceration or contusion (851).

(h) History of epidural, subdural, subarachnoid, or intercerebral hematoma (852).

(i) Associated abscess (326) or meningitis (958.8).

(j) Cerebrospinal fluid rhinorrhea (349.81) or otorrhea (388.61) persisting more than 7 days.

(k) Focal neurologic signs.

(l) Radiographic evidence of retained foreign body or bony fragments secondary to the trauma and/or operative procedure in the brain.

(m) Leptomeningeal cysts or Arteriovenous Fistula.

(2) History of moderate head injury (854.03) does not meet the standard. After 2 years post-injury, applicants may be qualified if neurological consultation shows no residual dysfunction or complications. Moderate head injuries are defined as unconsciousness, amnesia, or disorientation of person, place, or time alone or in combination, of more than 1 and less than 24-hours duration post-injury, or linear skull fracture.

(3) History of mild head injury (854.02) does not meet the standard. After 1 month post-injury, applicants may be qualified if neurological evaluation shows no residual dysfunction or complications. Mild head injuries are defined as a period of unconsciousness, amnesia, or disorientation of person, place, or time, alone or in combination of 1 hour or less post-injury.

(4) History of persistent post-traumatic symptoms (310.2) that interfere with normal activities or have duration of greater than 1 month does not meet the standard. Such symptoms include, but are not limited to headache, vomiting, disorientation, spatial disequilibrium, impaired memory, poor mental concentration, shortened attention span, dizziness, or altered sleep patterns.

g. Infectious diseases of the central nervous system.

(1) Current or history of acute infectious processes of the central nervous system, including, but not limited to meningitis (322), encephalitis (323), or brain abscess (324), do not meet the standard if occurring within 1 year before examination, or if there are residual neurological defects.

(2) History of neurosyphilis (094) of any form, including but not limited to general paresis, tabes dorsalis or meningovascular syphilis, does not meet the standard.

$h$. Current or history or narcolepsy or cataplexy (347) does not meet the standard.

i. Current or history of paralysis, weakness, lack of coordination, chronic pain, sensory disturbance, or other specified paralytic syndromes (344) does not meet the standard.

$j$. Epilepsy (345) occurring beyond the $6^{\text {th }}$ birthday, unless the applicant has been free of seizures for a period of 5 years while taking no medication for seizure control, and has a normal electroencephalogram (EEG) does not meet the standard. All such applicants will have a current neurology consultation with current EEG results.

k. Chronic nervous system disorders, including but not limited to myasthenia gravis (358.0), multiple sclerosis (340), and tic disorders (307.20) (for example, Tourett's (307.23)) do not meet the standard.

l. Current or history of retained central nervous system shunts of all kinds (V45.2) do not meet the standard.

\section{2-27. Learning, psychiatric and behavioral disorders}

a. Attention Deficit Disorder/Attention Deficit Hyperactivity Disorder (314), or Perceptual/Learning Disorder(s) (315) does not meet the standard, unless applicant can demonstrate passing academic performance and there has been no use of medication(s) in the previous 12 months.

$b$. Current or history of academic skills or perceptual defects (315) secondary to organic or functional mental disorders, including, but not limited to dyslexia, that interfere with school or employment, do not meet the standard. Applicants demonstrating passing academic and employment performance without utilization or recommendation of academic and/or work accommodations at any time in the previous 12 months may be qualified.

c. Current or history of disorders with psychotic features such as schizophrenia (295), paranoid disorder (297), and other unspecified psychosis (298) does not meet the standard.

d. Current mood disorders including, but not limited to, major depression (296.2-3), bipolar (296.4-7), affective psychoses (296.8-9), depressive not otherwise specified (311), do not meet the standard. 
(1) History of mood disorders requiring outpatient care for longer than 6 months by a physician or other mental health professional (V65.40), or inpatient treatment in a hospital or residential facility does not meet the standard.

(2) History of symptoms consistent with a mood disorder of a repeated nature that impairs school, social, or work efficiency does not meet the standard.

e. Current or history of adjustment disorders (309) within the previous 3 months does not meet the standard.

f. Current or history of conduct (312), or behavior (313) disorders does not meet the standard. Recurrent encounters with law enforcement agencies, antisocial attitudes or behaviors are tangible evidence of impaired capacity to adapt to military service and as such do not meet the standard.

g. Current or history of personality disorder (301) does not meet the standard. History (demonstrated by repeated inability to maintain reasonable adjustment in school, with employers or fellow workers, or other social groups), interview, or psychological testing revealing that the degree of immaturity, instability, personality inadequacy, impulsiveness, or dependency will likely interfere with adjustment in the Armed Forces does not meet the standard.

$h$. Current or history of other behavior disorders does not meet the standard, including, but not limited to conditions such as the following:

(1) Enuresis (307.6) or encopresis (307.7) after 13th birthday does not meet the standard.

(2) Sleepwalking (307.4) after 13th birthday does not meet the standard.

(3) Eating disorders (307.5), anorexia nervosa (307.1), bulimia (307.51), or unspecified disorders of eating (307.59) lasting longer than 3 months and occurring after 13th birthday do not meet the standard.

$i$. Any current receptive or expressive language disorder, including, but not limited to any speech impediment, stammering and stuttering (307.0) of such a degree as to significantly interfere with production of speech or to repeat commands, does not meet the standard.

$j$. History of suicidal behavior, including gesture(s) or attempt(s) (300.9), or history of self-mutilation, does not meet the standard.

k. Current or history of anxiety disorders (anxiety (300.01) or panic (300.2)), agoraphobia (300.21), social phobia (300.23), simple phobias (300.29), obsessive-compulsive (300.3), other acute reactions to stress (308), and posttraumatic stress disorder (309.81) do not meet the standard.

l. Current or history of dissociative disorders, including, but not limited to hysteria (300.1), depersonalization (300. 6), and other (300.8), do not meet the standard.

$m$. Current or history of somatoform disorders, including, but not limited to hypochondriasis (300.7) or chronic pain disorder, do not meet the standard.

$n$. Current or history of psychosexual conditions (302), including, but not limited to transsexualism, exhibitionism, transvestism, voyeurism, and other paraphilias, do not meet the standard.

$o$. Current or history of alcohol dependence (303), drug dependence (304), alcohol abuse (305), or other drug abuse (305.2 thru 305.9) does not meet the standard.

p. Current or history of other mental disorders (all 290-319 not listed above) that in the opinion of the civilian or military provider will interfere with, or prevent satisfactory performance of military duty, do not meet the standard.

\section{2-28. Skin and cellular tissues}

a. Current diseases of sebaceous glands to include severe acne (706.1), if extensive involvement of the neck, shoulders, chest, or back is present or would be aggravated by or interfere with the proper wearing of military equipment, do not meet the standard. Applicants under treatment with systemic retinoids, including, but not limited to isotretinoin (Accutane(r)) are disqualified until 8 (eight) weeks after completion of therapy.

$b$. Current or history of atopic dermatitis (691) or eczema (692) after the $9^{\text {th }}$ birthday does not meet the standard.

c. Current or history of contact dermatitis (692.4), especially involving materials used in any type of required protective equipment, does not meet the standard.

d. Cysts.

(1) Current cysts (706.2), (other than pilonidal cysts) of such a size or location as to interfere with the proper wearing of military equipment does not meet the standard.

(2) Current pilonidal cysts (685), if evidenced by the presence of a tumor mass or a discharging sinus does not meet the standard. Surgically resected pilonidal cyst that is symptomatic, unhealed, or less than 6 months post-operative does not meet the standard.

$e$. Current or history of bullous dermatoses (694), including, but not limited to dermatitis herpetiformis, pemphigus, and epidermolysis bullosa, does not meet the standard.

$f$. Current chronic lymphedema (457.1) does not meet the standard.

$g$. Current localized types of fungus infections (117), interfering with the proper wearing of military equipment or the performance of military duties, do not meet the standard. (For systemic fungal infections, refer to paragraph 2-30.)

$h$. Current or history of furunculosis or carbuncle (680), if extensive, recurrent, or chronic does not meet the standard.

$i$. Current or history of severe hyperhidrosis of hands or feet (780.8) does not meet the standard. 
j. Current or history of congenital (757) or acquired (216) anomalies of the skin such as nevi or vascular tumors that interfere with function, or are exposed to constant irritation do not meet the standard. History of Dysplastic Nevus Syndrome (232) does not meet the standard.

$k$. Current or history of keloid formation (701.4), if the tendency is marked or interferes with the proper wearing of military equipment, does not meet the standard.

l. Current lichen planus (697.0) does not meet the standard.

$m$. Current or history of neurofibromatosis (von Recklinghausen's disease) (237.7) does not meet the standard.

$n$. History of photosensitivity (692.72), including, but not limited to any primary sun-sensitive condition, such as polymorphous light eruption or solar urticaria; any dermatosis aggravated by sunlight such as lupus erythematosus does not meet the standard.

$o$. Current or history of psoriasis (696.1) does not meet the standard.

$p$. Current or history of radiodermatitis (692.82) does not meet the standard.

$q$. Current scars (709.2), or any other chronic skin disorder of a degree or nature that requires frequent outpatient treatment or hospitalization, which in the opinion of the certifying authority affects thermoregulatory function, or will interfere with the wearing of military clothing or equipment, or which exhibits a tendency to ulcerate, or interferes with the satisfactory performance of duty, do not meet the standard. Includes scars at skin graft donor or recipient sites. Scars at skin graft donor or recipient sites will include an evaluation of not only the relative total size of the burn wound, but also the measurable effects of the wound, the location of the wound and the risk of subsequent injury related to the wound itself.

(1) Prior burn injury (to include donor sites) involving a total body surface area of 40 percent or more does not meet the standard.

(2) Prior burn injury involving less than 40 percent total body surface area, which results in a loss or degradation of thermoregulatory function does not meet the standard. Examination will focus on the depth of the burn, anatomic location (extensive burns on the torso will most significantly impair heat dissipation), and destruction of sweat glands.

(3) Prior burn injury susceptible to trauma or resulting in functional impairment to such a degree as to interfere with the satisfactory performance of military duty, due to decreased range of motion, strength, or agility due to burn wound/ scarring does not meet the standard.

$r$. Current or history of extensive scleroderma (710.1) does not meet the standard.

s. Tattoos (709.9) that are otherwise prohibited under AR 670-1 do not meet the standard.

$t$. Current of history of chronic or recurrent urticaria (708.8) does not meet the standard.

$u$. Current symptomatic plantar wart(s) (078.19) does not meet the standard.

\section{2-29. Spine and sacroiliac joints}

a. Current or history of ankylosing spondylitis or other inflammatory spondylopathies (720) does not meet the standard. (See para 2-11a.)

$b$. Current or history of any condition, including, but not limited to the spine or sacroiliac joints, with or without objective signs that-

(1) Prevent the individual from successfully following a physically active vocation in civilian life (724) or that is associated with local or referred pain to the extremities, muscular spasm, postural deformities, or limitation of motion does not meet the standard.

(2) Require external support does not meet the standard.

(3) Require limitation of physical activity or frequent treatment does not meet the standard.

c. Current deviation or curvature of spine (737) from normal alignment, structure, or function does not meet the standard if:

(1) It prevents the individual from following a physically active vocation in civilian life.

(2) It interferes with the proper wearing of a uniform or military equipment.

(3) It is symptomatic.

(4) There is lumbar scoliosis greater than 20 degrees, thoracic scoliosis greater than 30 degrees, or kyphosis and lordosis greater than 55 degrees when measured by the Cobb method.

$d$. History of congenital fusion (756.15), involving more than two vertebral bodies does not meet the standard. Any surgical fusion of spinal vertebrae (P81.0) does not meet the standard.

$e$. Current or history of fractures or dislocation of the vertebrae (805) does not meet the standard. A compression fracture, involving less than 25 percent of a single vertebra is not disqualifying if the injury occurred more than 1 year before examination and the applicant is asymptomatic. A history of fractures of the transverse or spinous processes is not disqualifying if the applicant is asymptomatic.

$f$. History of juvenile epiphysitis (732.6) with any degree of residual change indicated by x-ray or kyphosis does not meet the standard.

g. Current herniated nucleus pulposus (722) or history of surgery to correct this condition does not meet the standard. 
$h$. Current or history of spina bifida (741) when symptomatic, if there is more than one vertebra level involved or with dimpling of the overlying skin does not meet the standard. History of surgical repair of spina bifida does not meet the standard.

i. Current or history of spondylolysis (congenital (756.11) or acquired (738.4)) and spondylolisthesis (congenital (756.12) or acquired (738.4)) do not meet the standard.

\section{2-30. Systemic diseases}

$a$. Current or history of disorders involving the immune mechanism including immunodeficiencies (279) does not meet the standard. Presence of Human Immunodeficiency Virus (HIV) or serologic evidence of infection (042) does not meet the standard. Positive Enzyme-Linked Immunoabsorbent Assay test(s) for HIV with ambiguous or inconclusive results on Western Blot testing does not meet the standard.

$b$. Current or history of lupus erythematosus (710.0) or mixed connective tissue disease variant (710.9) does not meet the standard.

c. Current or history of progressive systemic sclerosis (710.1), including CRST Variant, does not meet the standard. A single plaque of localized Scleroderma (morphea) that has been stable for at least 2 years is not disqualifying.

d. Current or history of Reiter's disease (099.3) does not meet the standard.

$e$. Current or history of rheumatoid arthritis (714.0) does not meet the standard.

$f$. Current or history of Sjögren's syndrome (710.2) does not meet the standard.

g. Current or history of vasculitis, including, but not limited to polyarteritis nodosa and allied conditions (446.0), arteritis (447.6), Behçet's (136.1), and Wegner's granulomatosis (446.4), does not meet the standard.

h. Tuberculosis (010)

(1) Current active tuberculosis or substantiated history of active tuberculosis in any form or location, regardless of past treatment, in the previous 2 years, does not meet the standard.

(2) Current residual physical or mental defects from past tuberculosis that will prevent the satisfactory performance of duty do not meet the standard.

(3) Individuals with a past history of active tuberculosis greater than 2 years before appointment, enlistment, or induction are qualified if they have received a complete course of standard chemotherapy for tuberculosis. Individuals with a tuberculin reaction in accordance with the guidelines of the American Thoracic Society and U.S. Public Health Service (ATS/USPHS), and without evidence of residual disease in pulmonary or non-pulmonary sites are eligible for enlistment induction, and appointment, provided they have received chemoprophylaxis in accordance with the guidelines of the ATS/USPHS.

(4) Current or history of untreated latent tuberculosis (positive Purified Protein Derivative with negative chest x-ray) (795.5) does not meet the standard.

i. Current untreated syphilis does not meet the standard (097).

$j$. History of anaphylaxis (995.0), including, but not limited to idiopathic and exercise-induced; anaphylaxis to venom, including stinging insects (989.5); foods or food additives (995.60-69); or to natural rubber latex (989.82), does not meet the standard.

$k$. Current residual of tropical fevers, including, but not limited to fevers, such as malaria (084) and various parasitic or protozoan infestations that prevent the satisfactory performance of military duty, does not meet the standard.

l. Current sleep disturbances (780.5), including, but not limited to sleep apneas, does not meet the standard.

$m$. History of malignant hyperthermia (995.86) does not meet the standard.

$n$. History of industrial solvent or other chemical intoxication (982) with sequelae does not meet the standard.

$o$. History of motion sickness (994.6) resulting in recurrent incapacitating symptoms or of such a severity to require pre-medication in the previous 3 years does not meet the standard.

$p$. History of rheumatic fever (390) does not meet the standard.

q. Current or history of muscular dystrophies (359) or myopathies does not meet the standard.

$r$. Current or history of amyloidosis (277.3) does not meet the standard.

$s$. Current or history of eosinophilic granuloma (277.8) does not meet the standard. Healed eosinophilic granuloma, when occurring as a single localized bony lesion and not associated with soft tissue or other involvement, will not be a cause for disqualification. All other forms of the Histiocytosis (202.3) do not meet the standard.

t. Current or history of polymyositis (710.4)/dermatomyositis complex (710.3) with skin involvement does not meet the standard.

$u$. History of rhabdomyolysis (728.88) does not meet the standard.

v. Current or history of sarcoidosis (135) does not meet the standard.

w. Current systemic fungus infections (117) do not meet the standard. For localized fungal infections, refer to paragraph $2-28 g$.

\section{2-31. Tumors and malignant diseases}

a. Current benign tumors (M8000), or conditions that interfere with function, prevent the proper wearing of the 
uniform or protective equipment, or will require frequent specialized attention, or have a high malignant potential, such as Dysplastic Nevus Syndrome, do not meet the standard.

$b$. Current or history of malignant tumors (V10) does not meet the standard. Skin cancer (other than malignant melanoma) removed with no residual, is not disqualifying.

\section{2-32. General and miscellaneous conditions and defects}

a. Current or history of parasitic diseases, if symptomatic or carrier state, including, but not limited to filariasis (125), trypanosomiasis (086), schistosomiasis (120), hookworm (uncinariasis) (126.9), and unspecified infectious and parasitic disease (136.9), do not meet the standard.

b. Current or history of other disorders, including, but not limited to cystic fibrosis (277.0), or porphyria (277.1), that prevent satisfactory performance of duty or require frequent or prolonged treatment do not meet the standard.

c. Current or history of cold-related disorders, including, but not limited to frostbite, chilblain, immersion foot (991), or cold urticaria (708.2), do not meet the standard. Current residual effects of cold-related disorders, including, but not limited to paresthesias, easily traumatized skin, cyanotic amputation of any digit, ankylosis, trench foot, or deep-seated ache, do not meet the standard.

d. History of angioedema, including hereditary angioedema (277.6), does not meet the standard.

$e$. History of receiving organ or tissue transplantation (V42) does not meet the standard.

$f$. History of pulmonary (415) or systemic embolization (444) does not meet the standard.

g. History of untreated acute or chronic metallic poisoning, including, but not limited to lead, arsenic, silver (985), beryllium, or manganese (985), does not meet the standard. Current complications or residual symptoms of such poisoning do not meet the standard.

$h$. History of heat pyrexia (992.0), heatstroke (992.0), or sunstroke (992.0) does not meet the standard. History of three or more episodes of heat exhaustion (992.3) does not meet the standard. Current or history of a predisposition to heat injuries, including disorders of sweat mechanism, combined with a previous serious episode does not meet the standard. Current or history of any unresolved sequelae of heat injury, including, but not limited to nervous, cardiac, hepatic or renal systems, does not meet the standard.

$i$. Current or history of any condition that in the opinion of the medical officer will significantly interfere with the successful performance of military duty or training does not meet the standard (should use specific ICD code whenever possible, or 796.9).

$j$. Any current acute pathological condition, including, but not limited to acute communicable diseases, until recovery has occurred without sequelae, does not meet the standard. 
Table 2-1

Military acceptable weight (in pounds) as related to age and height for males-Initial Army procurement, 2

\begin{tabular}{|c|c|c|c|c|c|}
\hline \multirow[b]{2}{*}{ Height (inches) } & \multirow[b]{2}{*}{$\begin{array}{c}\text { Minimum weight } \\
\text { any age } \\
\text { yielding a BMl of } 19\end{array}$} & \multicolumn{4}{|c|}{ Maximum weight by years of age } \\
\hline & & $17-20$ & $21-27$ & $28-39$ & 40 and over \\
\hline 60 & 97 & 139 & 141 & 143 & 146 \\
\hline 61 & 100 & 144 & 146 & 148 & 151 \\
\hline 62 & 104 & 148 & 150 & 153 & 156 \\
\hline 63 & 107 & 153 & 155 & 158 & 161 \\
\hline 64 & 110 & 158 & 160 & 163 & 166 \\
\hline 65 & 114 & 163 & 165 & 168 & 171 \\
\hline 66 & 117 & 168 & 170 & 173 & 177 \\
\hline 67 & 121 & 174 & 176 & 179 & 182 \\
\hline 68 & 125 & 179 & 181 & 184 & 187 \\
\hline 69 & 128 & 184 & 186 & 189 & 193 \\
\hline 70 & 132 & 189 & 192 & 195 & 199 \\
\hline 71 & 136 & 194 & 197 & 201 & 204 \\
\hline 72 & 140 & 200 & 203 & 206 & 210 \\
\hline 73 & 144 & 205 & 208 & 212 & 216 \\
\hline 74 & 148 & 211 & 214 & 218 & 222 \\
\hline 75 & 152 & 217 & 220 & 224 & 228 \\
\hline 76 & 156 & 223 & 226 & 230 & 234 \\
\hline 77 & 160 & 229 & 232 & 236 & 240 \\
\hline 78 & 164 & 235 & 238 & 242 & 247 \\
\hline 79 & 168 & 241 & 244 & 248 & 253 \\
\hline \multirow[t]{4}{*}{80} & 173 & 247 & 250 & 255 & 259 \\
\hline & & \multicolumn{4}{|c|}{ Maximum body fat by years of age } \\
\hline & & $17-20$ & $21-27$ & $28-39$ & 40 and over \\
\hline & & $26 \%$ & $26 \%$ & $28 \%$ & $30 \%$ \\
\hline
\end{tabular}

Notes:

${ }^{1}$ If a male exceeds these weights, percent body fat will be measured by the method described in AR 600-9.

2 If a male also exceeds this body fat, he will be rejected for service. 


\begin{tabular}{|c|c|c|c|c|c|}
\hline \multirow[b]{2}{*}{ Height (inches) } & \multirow[b]{2}{*}{$\begin{array}{c}\text { Minimum weight any } \\
\text { age } \\
\text { yielding a BMI of } 19\end{array}$} & \multicolumn{4}{|c|}{ Maximum weight by years of age } \\
\hline & & $17-20$ & $21-27$ & 28-39 & 40 and over \\
\hline 58 & 91 & 122 & 124 & 126 & 127 \\
\hline 59 & 94 & 127 & 128 & 130 & 131 \\
\hline 60 & 97 & 132 & 134 & 135 & 136 \\
\hline 61 & 100 & 136 & 137 & 139 & 141 \\
\hline 62 & 104 & 140 & 141 & 144 & 145 \\
\hline 63 & 107 & 145 & 147 & 148 & 149 \\
\hline 64 & 110 & 149 & 151 & 153 & 154 \\
\hline 65 & 114 & 154 & 156 & 158 & 160 \\
\hline 66 & 117 & 160 & 160 & 162 & 165 \\
\hline 67 & 121 & 163 & 166 & 168 & 169 \\
\hline 68 & 125 & 168 & 171 & 173 & 174 \\
\hline 69 & 128 & 173 & 176 & 178 & 180 \\
\hline 70 & 132 & 178 & 181 & 183 & 185 \\
\hline 71 & 136 & 183 & 186 & 188 & 191 \\
\hline 72 & 140 & 189 & 191 & 194 & 196 \\
\hline 73 & 144 & 194 & 196 & 200 & 202 \\
\hline 74 & 148 & 199 & 203 & 204 & 206 \\
\hline 75 & 152 & 205 & 208 & 210 & 212 \\
\hline 76 & 156 & 210 & 213 & 215 & 216 \\
\hline 77 & 160 & 216 & 219 & 221 & 223 \\
\hline 78 & 164 & 222 & 224 & 227 & 229 \\
\hline 79 & 168 & 227 & 230 & 234 & 236 \\
\hline \multirow[t]{4}{*}{80} & 173 & 233 & 236 & 240 & 241 \\
\hline & & \multicolumn{4}{|c|}{ Maximum body fat by years of age } \\
\hline & & $17-20$ & $21-27$ & 28-39 & 40 and over \\
\hline & & $32 \%$ & $32 \%$ & $34 \%$ & $36 \%$ \\
\hline
\end{tabular}

Notes:

1 If a female exceeds these weights, percent body fat will be measured by the method described in AR 600-9.

2 If a female also exceeds this body fat, she will be rejected for service.

\section{Chapter 3}

\section{Medical Fitness Standards for Retention and Separation, Including Retirement}

\section{3-1. General}

This chapter gives the various medical conditions and physical defects which may render a Soldier unfit for further military service and which fall below the standards required for the individuals in paragraph 3-2, below. These medical conditions and physical defects, individually or in combination, are those that-

a. Significantly limit or interfere with the Soldier's performance of their duties. 
$b$. May compromise or aggravate the Soldier's health or well-being if they were to remain in the military Service. This may involve dependence on certain medications, appliances, severe dietary restrictions, or frequent special treatments, or a requirement for frequent clinical monitoring.

c. May compromise the health or well-being of other Soldiers.

$d$. May prejudice the best interests of the Government if the individual were to remain in the military Service.

\section{3-2. Application}

These standards apply to the following individuals (see chaps 4 and 5 for other standards that apply to specific specialties):

a. All commissioned and warrant officers of the Active Army, ARNG/ARNGUS, and USAR.

$b$. All enlisted Soldiers of the Active Army, ARNG/ARNGUS, and USAR.

c. Students already enrolled in the HPSP and USUHS programs.

$d$. Enlisted Soldiers of the ARNG/ARNGUS or USAR who apply for enlistment in the Active Army.

e. Commissioned and warrant officers of the ARNG/ARNGUS or USAR who apply for appointment in the Active Army.

$f$. Soldiers of the ARNG/ARNGUS or USAR who re-enter active duty under the "split-training option." (However, the weight standards of tables $2-1$ and $2-2$ apply to split option trainees.)

$g$. Retired Soldiers recalled to active duty.

\section{3-3. Disposition}

Soldiers with conditions listed in this chapter who do not meet the required medical standards will be evaluated by an MEB as defined in AR 40-400 and will be referred to a PEB as defined in AR 635-40 with the following caveats:

a. USAR or ARNG/ARNGUS Soldiers not on active duty, whose medical condition was not incurred or aggravated during an active duty period, will be processed in accordance with chapter 9 and chapter 10 of this regulation.

$b$. Soldiers pending separation in accordance with provisions of AR 635-200 or AR 600-8-24 authorizing separation under other than honorable conditions who do not meet medical retention standards will be referred to an MEB. In the case of enlisted Soldiers, the physical disability processing and the administrative separation processing will be conducted in accordance with the provisions of AR 635-200 and AR 635-40. In the case of commissioned or warrant officers, the physical disability processing and the administrative separation processing will be conducted in accordance with the provisions of AR 600-8-24 and AR 635-40.

c. A Soldier will not be referred to an MEB or a PEB because of impairments that were known to exist at the time of acceptance in the Army and that have remained essentially the same in degree of severity and have not interfered with successful performance of duty.

$d$. Physicians who identify Soldiers with medical conditions listed in this chapter should initiate an MEB at the time of identification. Physicians should not defer initiating the MEB until the Soldier is being processed for nondisability retirement. Many of the conditions listed in this chapter (for example, arthritis in para 3-14b) fall below retention standards only if the condition has precluded or prevented successful performance of duty. In those cases when it is clear the condition is long standing and has not prevented the Soldier from reaching retirement, then the Soldier meets the standard and an MEB is not required.

e. Soldiers who have previously been found unfit for duty by a PEB, but were continued on active duty (COAD) under the provisions of AR 635-40, chapter 6, will be referred to a PEB prior to retirement or separation processing.

$f$. If the Secretary of Defense prescribes less stringent standards during partial or full mobilization, individuals who meet the less stringent standards but do not meet the standards of this chapter will not be referred for an MEB or a PEB, until the termination of the mobilization or as directed by the Secretary of the Army.

\section{3-4. General policy}

Possession of one or more of the conditions listed in this chapter does not mean automatic retirement or separation from the Service. Physicians are responsible for referring Soldiers with conditions listed below to an MEB. It is critical that MEBs are complete and reflect all of the Soldier's medical problems and physical limitations. The PEB will make the determination of fitness or unfitness. The PEB, under the authority of the U.S. Army Physical Disability Agency, will consider the results of the MEB, as well as the requirements of the Soldier's MOS, in determining fitness. (See chapter 9 and chapter 10 of this regulation for processing of RC Soldiers.)

\section{3-5. Abdominal and gastrointestinal defects and diseases}

The causes for referral to an MEB are as follows:

a. Achalasia (cardiospasm) with dysphagia not controlled by dilatation or surgery, continuous discomfort, or inability to maintain weight.

$b$. Amoebic abscess with persistent abnormal liver function tests and failure to maintain weight and vigor after appropriate treatment.

c. Biliary dyskinesia with frequent abdominal pain not relieved by simple medication, or with periodic jaundice. 
d. Cirrhosis of the liver with recurrent jaundice, ascites, or demonstrable esophageal varices or history of bleeding therefrom.

$e$. Gastritis, if severe, chronic hypertrophic gastritis with repeated symptomatology and hospitalization, confirmed by gastroscopic examination.

$f$. Hepatitis, $\mathrm{B}$ or $\mathrm{C}$, chronic, when following the acute stage, symptoms persist, and there is objective evidence of impairment of liver function. Chronic hepatitis B as documented by positive hepatitis B surface or e antigen or detectable hepatitis B Deoxyribonucleic acid (DNA) viral load in serum. Chronic hepatitis C as documented by detectable hepatitis C RNA viral load in serum.

g. Hernia, including inguinal, and other abdominal, except for small asymptomatic umbilical, with severe symptoms not relieved by dietary or medical therapy, or recurrent bleeding in spite of prescribed treatment or other hernias if symptomatic and if operative repair is contraindicated for medical reasons or when not amenable to surgical repair.

$h$. Crohn's Disease/Ileitis, regional, except when responding well to treatment.

$i$. Pancreatitis, chronic, with frequent abdominal pain of a severe nature; steatorrhea or disturbance of glucose metabolism requiring hypoglycemic agents.

j. Peritoneal adhesions with recurring episodes of intestinal obstruction characterized by abdominal colicky pain, vomiting, and intractable constipation requiring frequent admissions to the hospital.

$k$. Proctitis, chronic, with moderate to severe symptoms of bleeding, painful defecation, tenesmus, and diarrhea, and repeated admissions to the hospital.

l. Ulcer, duodenal, or gastric with repeated hospitalization, or "sick in quarters" because of frequent recurrence of symptoms (pain, vomiting, or bleeding) in spite of good medical management and supported by endoscopic evidence of activity.

$m$. Ulcerative colitis, except when responding well to treatment.

$n$. Rectum, stricture of with severe symptoms of obstruction characterized by intractable constipation, pain on defecation, or difficult bowel movements, requiring the regular use of laxatives or enemas, or requiring repeated hospitalization.

\section{3-6. Gastrointestinal and abdominal surgery}

The causes for referral to an MEB are as follows:

a. Colectomy, partial or total, when more than mild symptoms of diarrhea remain or if complicated by colostomy.

b. Colostomy, when permanent.

c. Enterostomy, when permanent.

d. Gastrectomy, total.

e. Gastrectomy, subtotal, with or without vagotomy, or gastrojejunostomy, with or without vagotomy, when, in spite of good medical management, the individual develops "dumping syndrome" which persists for 6 months postoperatively; or develops frequent episodes of epigastric distress with characteristic circulatory symptoms or diarrhea persisting 6 months postoperatively; or continues to demonstrate appreciable weight loss 6 months postoperatively.

$f$. Gastrostomy, when permanent.

g. Ileostomy, when permanent.

h. Pancreatectomy.

$i$. Pancreaticoduodenostomy, pancreaticogastrostomy, or pancreaticojejunostomy, followed by more than mild symptoms of digestive disturbance, or requiring insulin.

j. Proctectomy.

$k$. Proctopexy, proctoplasty, proctorrhaphy, or proctotomy, if fecal incontinence remains after an appropriate treatment period.

\section{3-7. Blood and blood-forming tissue diseases}

The causes for referral to an MEB are as follows:

a. Anemia, hereditary, acquired, aplastic, or unspecified, when response to therapy is unsatisfactory, or when therapy is such as to require prolonged, intensive medical supervision.

$b$. Hemolytic crisis, chronic and symptomatic.

c. Leukopenia, chronic, when response to therapy is unsatisfactory, or when therapy is such as to require prolonged, intensive medical supervision.

d. Hypogammaglobulinemia with objective evidence of function deficiency and severe symptoms not controlled with treatment.

$e$. Purpura and other bleeding diseases, when response to therapy is unsatisfactory, or when therapy is such as to require prolonged, intensive medical supervision.

$f$. Thromboembolic disease when response to therapy is unsatisfactory, or when therapy is such as to require prolonged, intensive medical supervision.

g. Splenomegaly, chronic. 
$h$. HIV confirmed antibody positivity, with the presence of progressive clinical illness or immunological deficiency. For Active Army Soldiers and RC Soldiers on active duty for more than 30 days (except for training under 10 USC 10148), an MEB must be accomplished and, if appropriate, the Soldier must be referred to a PEB under AR 635-40. For RC Soldiers not on active duty for more than 30 days or on ADT under 10 USC 10148, referral to a PEB will be determined under AR 635-40. Records of official diagnoses provided by private physicians (that is, civilian doctors providing evaluations under contract with Department of the Army (DA) or DOD, or civilian public health officials) concerning the presence of progressive clinical illness or immunological deficiency in RC Soldiers may be used as a basis for administrative action under, for example, AR 135-133, AR 135-175, AR 135-178, or AR 140-10, as appropriate. (See AR 600-110 for HIV policies, including testing requirements.)

\section{3-8. Dental diseases and abnormalities of the jaws}

The causes for referral to an MEB are diseases of the jaws, periodontium, or associated tissues when, following restorative surgery, there are residuals that are incapacitating or interfere with the individual's satisfactory performance of military duty.

\section{3-9. Ears}

The causes for referral to an MEB are as follows:

$a$. Infections of the external auditory canal when chronic and severe, resulting in thickening and excoriation of the canal or chronic secondary infection requiring frequent and prolonged medical treatment and hospitalization.

$b$. Malfunction of the acoustic nerve. (Evaluate functional impairment of hearing under para 3-10.)

c. Mastoiditis, chronic, with constant drainage from the mastoid cavity, requiring frequent and prolonged medical care.

$d$. Mastoiditis, chronic, following mastoidectomy, with constant drainage from the mastoid cavity, requiring frequent and prolonged medical care or hospitalization.

$e$. Ménière's syndrome or any peripheral imbalance, syndrome or labyrinthine disorder with recurrent attacks of sufficient frequency and severity as to interfere with the satisfactory performance of duty or requiring frequent or prolonged medical care or hospitalization.

$f$. Otitis media, moderate, chronic, suppurative, resistant to treatment, and necessitating frequent and prolonged medical care or hospitalization.

\section{3-10. Hearing}

The causes for referral to an MEB are as follows:

a. Soldiers incapable of performing their military duties with a hearing aid (see para 8-27).

$b$. Soldiers who fail the Speech Recognition In Noise Test (SPRINT).

c. Soldiers with a permanent $\mathrm{H} 4$ hearing profile.

\section{3-11. Endocrine and metabolic disorders}

The causes for referral to an MEB are as follows:

a. Acromegaly.

$b$. Adrenal insufficiency requiring replacement therapy.

c. Diabetes insipidus requiring the use of medication for control.

$d$. Diabetes mellitus, unless hemoglobin A1c can be maintained at <(less than) $7 \%$ using only lifestyle modifications (diet, exercise).

$e$. Goiter causing breathing obstruction.

$f$. Gout in advanced cases with frequent acute exacerbations and severe bone, joint, or kidney damage.

g. Fasting hypoglycemia (as documented during a 72-hour fast) when caused by an insulinoma or other hypoglycemia-inducing tumor.

$h$. Hyperparathyroidism when residuals or complications of surgical correction such as renal disease or bony deformities preclude the reasonable performance of military duty.

$i$. Cushing's syndrome.

$j$. Osteomalacia or osteoporosis resulting in fracture with residuals after therapy of such nature or degree as to preclude the satisfactory performance of duty.

$k$. Primary hyperaldosteronism when resulting in uncontrolled hypertension and/or hypokalemia.

l. Multiple endocrine neoplasia, any type.

$m$. Pituitary macroadenomas when resulting in hypothalamic/pituitary dysfunction or symptoms of mass effect.

$n$. Pheochromocytoma.

$o$. Thyroid carcinoma, any type, if persistent despite usual therapy (surgery, radioactive iodine and treatment with suppressive doses of levothyroxine).

$p$. Hypoparathyroidism, when severe, persistent, and difficult to manage. 
q. Salt-wasting congenital adrenal hyperplasia.

$r$. Carcinoid syndrome.

$s$. Endocrine tumors of the gastrointestinal tract, when response to therapy is unsatisfactory, or when therapy is such as to require prolonged, intensive medical supervision. Such tumors include gastrinoma, glucagonoma, vipoma, neurotensinoma, PPoma, and somatostatinoma.

\section{3-12. Upper extremities}

The causes for referral to an MEB are as follows (see also para 3-14):

a. Amputation.

(1) For purposes of this regulation, upper extremity amputation is defined as the loss of part or parts of an upper extremity equal to or greater than--

(a) A thumb proximal to the interphalangeal joint.

(b) Two fingers of one hand, other than the little finger, at the proximal interphalangeal joints.

(c) One finger, other than the little finger, at the metacarpophalangeal joint and the thumb of the same hand at the interphalangeal joint.

(2) Soldiers with amputations will (assuming no other disqualifying medical conditions) be provided a temporary profile not less than 4 months (but not to exceed 1 year) to enable the Soldier to attain maximum medical benefit.

$b$. Joint ranges of motion (ROM) which do not equal or exceed the measurements listed below. Measurements should be made with a goniometer (a bubble goniometer/inclinometer is also acceptable) and conform to the methods illustrated and described in the Veterans Administration Schedule for Rating Disabilities (VASRD). SF Form 527 (Medical Record-Group Muscle Strength, Joint R.O.M. Girth and Length Measurements) should be used to document the ROM and the method of measurement.

(1) Shoulder-forward elevation to 90 degrees, or abduction to 90 degrees.

(2) Elbow-flexion to 100 degrees, or extension to 60 degrees.

(3) Wrist - a total range extension plus flexion of 15 degrees.

(4) Hand (for this purpose, combined joint motion is the arithmetic sum of the motion at each of the three finger joints (VASRD)) - an active flexor value of combined joint motions of 135 degrees in each of two or more fingers of the same hand, or an active extensor value of combined joint motions of 75 degrees in each of the same two or more fingers, or limitation of motion of the thumb that precludes opposition to at least two finger tips.

$c$. Recurrent dislocations of the shoulder, when not repairable or surgery is contradicated.

\section{3-13. Lower extremities}

The causes for referral to an MEB are as follows (see also para 3-14):

a. Amputations.

(1) Lower extremity amputations are defined, for purposes of this regulation, as follows:

(a) Loss of toes that precludes the abilities to run or walk without a perceptible limp and to engage in fairly strenuous jobs.

(b) Any loss greater than that specified above to include foot, ankle, below the knee, above the knee, femur, hip.

(2) Soldiers with amputations will (assuming no other disqualifying medical conditions) be provided a temporary profile not less than 4 months (but not to exceed 1 year) to enable the Soldier to attain maximum medical benefit.

b. Feet.

(1) Hallux valgus when moderately severe, with exostosis or rigidity and pronounced symptoms; or severe with arthritic changes.

(2) Pes planus, when symptomatic, more than moderate, with pronation on weight bearing which prevents the wearing of military footwear, or when associated with vascular changes.

(3) Pes cavus when moderately severe, with moderate discomfort on prolonged standing and walking, metatarsalgia, and which prevents the wearing of military footwear.

(4) Neuroma that is refractory to medical treatment, refractory to surgical treatment, and interferes with the satisfactory performance of military duties.

(5) Plantar fascitis or heel spur syndrome that is refractory to medical or surgical treatment, interferes with the satisfactory performance of military duties, or prevents the wearing of military footwear.

(6) Hammertoes, severe, that precludes the wearing of appropriate military footwear, refractory to surgery, or interferes with satisfactory performance of duty.

(7) Hallux limitus, hallux rigidus.

c. Internal derangement of the knee.

(1) Residual instability following remedial measures, if more than moderate in degree.

(2) If complicated by arthritis, see paragraph 3-14a.

d. Joint ranges of motion (ROM). ROM that does not equal or exceed the measurements listed below. Measurements 
should be made with a goniometer (a bubble goniometer/inclinometer is also acceptable) and conform to the methods illustrated and described in the VASRD.

(1) Hip-flexion to 90 degrees or extension to 0 degree.

(2) Knee-flexion to 90 degrees or extension to 15 degrees.

(3) Ankle-dorsiflexion to 10 degrees or planter flexion to 10 degrees.

$e$. Shortening of an extremity that exceeds 2 inches.

$f$. Recurrent dislocations of the patella.

(See also para 3-14.)

\section{3-14. Miscellaneous conditions of the extremities}

The causes for referral to an MEB are as follows (see also paras 3-12 and 3-13):

a. Arthritis due to infection, associated with persistent pain and marked loss of function with objective x-ray evidence and documented history of recurrent incapacity for prolonged periods. For arthritis due to gonococcic or tuberculous infection, see paragraphs $3-40 j$ and $3-45 b$.

$b$. Arthritis due to trauma, when surgical treatment fails or is contraindicated and there is functional impairment of the involved joints so as to preclude the satisfactory performance of duty.

$c$. Osteoarthritis, with severe symptoms associated with impairment of function, supported by x-ray evidence and documented history of recurrent incapacity for prolonged periods.

$d$. Avascular necrosis of bone when severe enough to prevent successful performance of duty.

$e$. Chondromalacia or osteochondritis dissecans, severe, manifested by frequent joint effusion, more than moderate interference with function, or with severe residuals from surgery.

$f$. Fractures.

(1) Malunion of fractures, when, after appropriate treatment, there is more than moderate malunion with marked deformity and more than moderate loss of function.

(2) Nonunion of fractures, when, after an appropriate healing period, the nonunion precludes satisfactory performance of duty.

(3) Bone fusion defect, when manifested by more than moderate pain and loss of function.

(4) Callus, excessive, following fracture, when functional impairment precludes satisfactory performance of duty and the callus does not respond to adequate treatment.

g. Joints.

(1) Arthroplasty with severe pain, limitation of motion, and of function.

(2) Bony or fibrous ankylosis, with severe pain involving major joints or spinal segments in an unfavorable position, and with marked loss of function.

(3) Contracture of joint, with marked loss of function and the condition is not remediable by surgery.

(4) Loose bodies within a joint, with marked functional impairment and complicated by arthritis to such a degree as to preclude favorable results of treatment or not remediable by surgery.

(5) Prosthetic replacement of major joints if there is resultant loss of function or pain that precludes satisfactory performance of duty.

$h$. Muscles.

(1) Flaccid paralysis of one or more muscles with loss of function that precludes satisfactory performance of duty following surgical correction or if not remediable by surgery.

(2) Spastic paralysis of one or more muscles with loss of function that precludes the satisfactory performance of military duty.

i. Myotonia congenita.

$j$. Osteitis deformans (Paget's disease) with involvement of single or multiple bones with resultant deformities or symptoms severely interfering with function.

$k$. Osteoarthropathy, hypertrophic, secondary with moderately severe to severe pain present, with joint effusion occurring intermittently in one or multiple joints, and with at least moderate loss of function.

$l$. Osteomyelitis, chronic, with recurrent episodes not responsive to treatment and involving the bone to a degree that interferes with stability and function.

$m$. Tendon transplant with fair or poor restoration of function with weakness that seriously interferes with the function of the affected part.

(See also paras 3-12 and 3-13.)

$n$. Tendinopathy. Any tendonitis, tenosynovitis, or tendinopathy that precludes satisfactory performance of military duties.

\section{3-15. Eyes}

The causes for referral to an MEB are as follows: 
a. Active eye disease or any progressive organic disease or degeneration, regardless of the stage of activity, that is resistant to treatment and affects the distant visual acuity or visual fields so that distant visual acuity does not meet the standard stated in paragraph 3-16e or the diameter of the field of vision in the better eye is less than 20 degrees.

b. Aphakia, bilateral.

c. Atrophy of the optic nerve due to disease.

$d$. Glaucoma, if resistant to treatment or affecting visual fields as in a above, or if side effects of required medication are functionally incapacitating.

$e$. Degenerations, when vision does not meet the standards of paragraph 3-16e, or when vision is correctable only by the use of contact lenses or other special corrective devices (telescopic lenses, etc.).

$f$. Diseases and infections of the eye, when chronic, more than mildly symptomatic, progressive, and resistant to treatment after a reasonable period. This includes intractable allergic conjunctivitis inadequately controlled by medications and immunotherapy.

g. Residuals or complications of injury or disease, when progressive or when reduced visual acuity does not meet the criteria stated in paragraph 3-16e.

$h$. Unilateral detachment of retina if any of the following exists:

(1) Visual acuity does not meet the standard stated in paragraph 3-16e.

(2) The visual field in the better eye is constricted to less than 20 degrees.

(3) Uncorrectable diplopia exists.

(4) Detachment results from organic progressive disease or new growth, regardless of the condition of the better eye.

$i$. Bilateral detachment of retina, regardless of etiology or results of corrective surgery.

\section{3-16. Vision}

The causes for referral to an MEB are as follows:

a. Aniseikonia, with subjective eye discomfort, neurologic symptoms, sensations of motion sickness and other gastrointestinal disturbances, functional disturbances and difficulties in form sense, and not corrected by iseikonica lenses.

b. Binocular diplopia, not correctable by surgery, that is severe, constant, and in a zone less than 20 degrees from the primary position.

c. Hemianopsia, of any type if bilateral, permanent, and based on an organic defect. Those due to a functional neurosis and those due to transitory conditions, such as periodic migraine, are not considered to fall below required standards.

$d$. Night blindness, of such a degree that the Soldier requires assistance in any travel at night.

e. Visual acuity.

(1) Vision that cannot be corrected with ordinary spectacle lenses (contact lenses or other special corrective devices (telescopic lenses, and so forth) are unacceptable) to at least: 20/40 in one eye and 20/100 in the other eye, or 20/30 in one eye and 20/200 in the other eye, or 20/20 in one eye and 20/800 in the other eye, or

(2) An eye has been enucleated.

$f$. Visual field with bilateral concentric constriction to less than 20 degrees.

\section{3-17. Genitourinary system}

The causes for referral to an MEB are as follows:

a. Cystitis, when complications or residuals of treatment themselves preclude satisfactory performance of duty.

$b$. Dysmenorrhea, when symptomatic, irregular cycle, not amenable to treatment, and of such severity as to necessitate recurrent absences of more than 1 day.

c. Endometriosis, symptomatic and incapacitating to a degree that necessitates recurrent absences of more than 1 day.

$d$. Hypospadias, when accompanied by evidence of chronic infection of the genitourinary tract or instances where the urine is voided in such a manner as to soil clothes or surroundings and the condition is not amenable to treatment.

$e$. Incontinence of urine, due to disease or defect not amenable to treatment and of such severity as to necessitate recurrent absence from duty.

$f$. Kidney.

(1) Calculus in kidney, when bilateral, resulting in frequent or recurring infections, or when there is evidence of obstructive uropathy not responding to medical or surgical treatment.

(2) Congenital anomaly, when bilateral, resulting in frequent or recurring infections, or when there is evidence of obstructive uropathy not responding to medical or surgical treatment.

(3) Cystic kidney (polycystic kidney), when symptomatic and renal function is impaired or is the focus of frequent infection.

(4) Glomerulonephritis, when chronic. 
(5) Hydronephrosis, when more than mild, bilateral, and causing continuous or frequent symptoms.

(6) Hypoplasia of the kidney, when symptomatic and associated with elevated blood pressure or frequent infections and not controlled by surgery.

(7) Nephritis, when chronic.

(8) Nephrosis.

(9) Perirenal abscess, with residuals of a degree that precludes the satisfactory performance of duty.

(10) Pyelonephritis or pyelitis, when chronic, that has not responded to medical or surgical treatment, with evidence of hypertension, eye-ground changes, cardiac abnormalities.

(11) Pyonephrosis, when not responding to treatment.

g. Menopausal syndrome, physiologic or artificial, when symptoms are not amenable to treatment and preclude successful performance of duty.

$h$. Chronic pelvic pain with or without demonstrative pathology that has not responded to medical or surgical treatment and of such severity to necessitate recurrent absence from duty.

$i$. Strictures of the urethra or ureter, when severe and not amenable to treatment.

$j$. Urethritis, chronic, when not responsive to treatment and necessitating frequent absences from duty.

\section{3-18. Genitourinary and gynecological surgery}

The causes for referral to an MEB are as follows:

a. Cystectomy.

$b$. Cystoplasty, if reconstruction is unsatisfactory or if residual urine persists in excess of 50 cubic centimeters or if refractory symptomatic infection persists.

c. Hysterectomy, when residual symptoms or complications preclude the satisfactory performance of duty.

$d$. Nephrectomy, when after treatment, there is infection or pathology in the remaining kidney.

$e$. Nephrostomy, if drainage persists.

$f$. Oophorectomy, when complications or residual symptoms are not amenable to treatment and preclude successful performance of duty.

g. Pyelostomy, if drainage persists.

$h$. Ureterocolostomy.

$i$. Ureterocystostomy, when both ureters are markedly dilated with irreversible changes.

$j$. Ureteroileostomy cutaneous.

k. Ureteroplasty.

(1) When unilateral procedure is unsuccessful and nephrectomy is necessary, consider it on the basis of the standard for a nephrectomy; or

(2) When bilateral, evaluate residual obstruction or hydronephrosis and consider it on the basis of the residuals involved.

l. Ureterosigmoidostomy.

$m$. Ureterostomy, external or cutaneous.

$n$. Urethrostomy, if there is complete amputation of the penis or when a satisfactory urethra cannot be restored.

$o$. Kidney transplant recipient. If found fit for duty by a PEB, Soldiers should be restricted to assignment locations where adequate medical care is available and should not deploy to an austere environment. Such Soldiers should not wear individual chemical equipment due to possible drug interactions.

\section{3-19. Head}

A skull defect that poses a danger to the Soldier or interferes with the wearing of protective headgear is cause for referral to an MEB.

\section{3-20. Neck}

The causes for referral to an MEB are torticollis (wry neck); severe fixed deformity with cervical scoliosis, flattening of the head and face, and loss of cervical mobility. (See also paras 3-11 and 3-39h.)

\section{3-21. Heart}

The causes for referral to an MEB are as follows (see table 3-1 for functional classifications and for metabolic equivalents (METS) ratings to be included in the MEB):

a. Coronary heart disease associated with-

(1) Myocardial infarction, angina pectoris, or congestive heart failure due to fixed obstructive coronary artery disease or coronary artery spasm. The policies for trial of duty, profiling, and referral to an MEB and a PEB (as outlined in para 3-25) apply. The trial of duty will be for 120 days.

(2) Myocardial infarction with normal coronary artery anatomy. The policies for trial of duty, profiling, and referral to an MEB and a PEB (as outlined in para 3-25) apply. The trial of duty will be for 120 days. 
(3) Angina pectoris in association with objective evidence of myocardial ischemia in the presence of normal coronary artery anatomy.

(4) Fixed obstructive coronary artery disease, asymptomatic but with objective evidence of myocardial ischemia. The policies for trial of duty, profiling, and referral to an MEB and a PEB (as outlined in para 3-25) apply. The trial of duty will be for 120 days.

$b$. Supraventricular tachyarrhythmias, when life threatening or symptomatic enough to interfere with performance of duty and when not adequately controlled. This includes atrial fibrillation, atrial flutter, paroxysmal supraventricular tachycardia, and others.

c. Endocarditis with any residual abnormality or if associated with valvular, congenital, or hypertrophic myocardial disease.

d. Heart block (second degree or third degree AV block) and symptomatic bradyarrhythmias, even in the absence of organic heart disease or syncope. Wenckebach second degree heart block occurring in healthy asymptomatic individuals without evidence of organic heart disease is not a cause for referral to a PEB. None of these conditions is cause for $\mathrm{MEB} / \mathrm{PEB}$ when associated with recognizable temporary precipitating conditions: for example, perioperative period, hypoxia, electrolyte disturbance, drug toxicity, acute illness.

e. Myocardial disease, New York Heart Association or Canadian Cardiovascular Society Functional Class II or worse. (See table 3-1.)

$f$. Ventricular flutter and fibrillation, ventricular tachycardia when potentially life threatening (for example, when associated with forms of heart disease that are recognized to predispose to increased risk of death and when there is no definitive therapy available to reduce this risk) or when symptomatic enough to interfere with the performance of duty. None of these ventricular arrhythmias are a cause for medical board referral to a PEB when associated with recognizable temporary precipitating conditions: for example, perioperative period, hypoxia, electrolyte disturbance, drug toxicity, or acute illness.

g. Sudden cardiac death, when an individual survives sudden cardiac death that is not associated with a temporary or treatable cause, and when there is no definitive therapy available to reduce the risk of recurrent sudden cardiac death.

$h$. Hypertrophic cardiomyopathy when it restricts activity.

$i$. Pericarditis as follows:

(1) Chronic constrictive pericarditis unless successful remedial surgery has been performed.

(2) Chronic serous pericarditis.

j. Valvular heart disease with cardiac insufficiency at functional capacity of Class II or worse as defined by the New York Heart Association. (See table 3-1.)

$k$. Ventricular premature contractions with frequent or continuous attacks, whether or not associated with organic heart disease, accompanied by discomfort or fear of such a degree as to interfere with the satisfactory performance of duty.

l. Recurrent syncope or near syncope of cardiovascular etiology that is not controlled or when it interferes with the performance of duty, even if the etiology is unknown.

$m$. Any cardiovascular disorder requiring chronic drug therapy in order to prevent the occurrence of potentially fatal or severely symptomatic events that would interfere with duty performance.

$n$. Congenital heart disease that has long term risks, complications, or impact on duty performance. The exception would be those congenital heart disease conditions that can be repaired with resolution of long term risks, complications, and impact on duty performance.

\section{3-22. Vascular system}

The causes for referral to an MEB are as follows:

a. Arteriosclerosis obliterans when any of the following pertain:

(1) Intermittent claudication of sufficient severity to produce discomfort and inability to complete a walk of 200 yards or less on level ground at 112 steps per minute without a rest.

(2) Objective evidence of arterial disease with symptoms of claudication, ischemic rest pain, or with gangrenous or ulcerative skin changes of a permanent degree in the distal extremity.

(3) Involvement of more than one organ, system, or anatomic region (the lower extremities comprise one region for this purpose) with symptoms of arterial insufficiency.

b. Major cardiovascular anomalies including coarctation of the aorta, unless satisfactorily treated by surgical correction or other newly developed techniques, and without any residual abnormalities or complications.

c. Aneurysm of any vessel not correctable by surgery and aneurysm corrected by surgery after a period of up to 90 days trial of duty that results in the individual's inability to perform satisfactory duty. The policies for trial of duty, profiling, and referral to an MEB and a PEB (as outlined in para 3-25) apply.

d. Periarteritis nodosa with definite evidence of functional impairment.

$e$. Chronic venous insufficiency (postphlebitic syndrome) when more than mild and symptomatic despite elastic support. 
$f$. Raynaud's phenomenon manifested by trophic changes of the involved parts characterized by scarring of the skin or ulceration.

g. Thromboangiitis obliterans with intermittent claudication of sufficient severity to produce discomfort and inability to complete a walk of 200 yards or less on level ground at 112 steps per minute without rest, or other complications.

$h$. Thrombophlebitis when repeated attacks requiring treatment are of such frequency as to interfere with the satisfactory performance of duty.

$i$. Varicose veins that are severe and symptomatic despite therapy.

j. Cold injury. (See paragraph 3-46).

\section{3-23. Miscellaneous cardiovascular conditions}

The causes for referral to an MEB are as follows:

$a$. Hypertensive cardiovascular disease and hypertensive vascular disease. Diastolic pressure consistently more than $110 \mathrm{mmHg}$ following an adequate period of therapy in an ambulatory status.

$b$. Rheumatic fever, active, with heart damage. Recurrent attacks.

3-24. Surgery and other invasive procedures involving the heart, pericardium, or vascular system These procedures include newly developed techniques or prostheses not otherwise covered in this paragraph. The causes for referral to an MEB are as follows:

a. Permanent prosthetic valve implantation.

$b$. Implantation of permanent pacemakers, antitachycardia and defibrillator devices, and similar newly developed devices.

c. Reconstructive cardiovascular surgery employing exogenous grafting material.

$d$. Vascular reconstruction, after a period of 90 days trial of duty when medically advisable, that results in the individual's inability to perform satisfactory duty. The policies for trial of duty, profiling, and referral to an MEB and a PEB (as outlined in para 3-25) apply.

$e$. Coronary artery revascularization, with the option of a 120-day trial of duty based upon physician recommendation when the individual is asymptomatic, without objective evidence of myocardial ischemia, and when other functional assessment (such as exercise testing and newly developed techniques) indicates that it is medically advisable. Any individual undergoing median sternotomy for surgery will be restricted from lifting 25 pounds or more, performing pullups and pushups, or as otherwise prescribed by a physician for a period of 90 days from the date of surgery on DA Form 3349 (Physical Profile). The policies for trial of duty, profiling, and referral to an MEB and a PEB (as outlined in para 3-25) apply.

$f$. Heart or heart-lung transplantation.

g. Coronary or valvular angioplasty procedures, with the option of a 180-day trial of duty based upon physician recommendation when the individual is asymptomatic, without objective evidence of myocardial ischemia, and when other functional assessment (such as cardiac catheterization, exercise testing, and newly developed techniques) indicates that it is medically advisable. The policies for trial of duty, profiling, and referral to an MEB and a PEB (as outlined in para 3-25) apply.

$h$. Cardiac arrhythmia ablation procedures, with the option of a 180-day trial of duty based upon physician recommendation when asymptomatic, and no evidence of any unfitting arrhythmia as noted in paragraph 3-21. The policies for trial of duty, MEB, and physical profile (as outlined in para 3-25) apply.

$i$. Congenital heart disease with surgical or percutaneous repair procedures, with the option of a 180-day trial of duty based upon physician recommendations when the individual is asymptomatic and when other functional assessment procedures indicate it is advisable. The policies for trial of duty and referral to an MEB are outlined in paragraph 3-25.

\section{3-25. Trial of duty and profiling for cardiovascular conditions}

a. Trial of duty will be based upon a cardiologist's recommendation when the individual is asymptomatic without objective evidence of myocardial ischemia, and when other functional assessments (such as coronary angiography, exercise testing, and newly developed techniques) indicate it is medically advisable.

$b$. Prior to commencing the trial of duty period, an evaluation will be accomplished in all cases and a physical activity prescription on DA Form 3349 will be provided by the cardiologist. The results of the trial of duty will include the individual's interim history, present condition, prognosis, and the final recommendations. If the Soldier successfully completes the trial of duty, is considered a New York Heart Association Functional Class I, AND there are no physical or assignment restrictions, the Soldier may be returned to duty without referral to a MEB. If the Soldier has any physical restrictions after the trial of duty; he/she should be referred to an MEB. In addition to the documented results of the trial of duty, a detailed report from the commander or supervisor clearly describing the individual's ability to accomplish assigned duties and to perform physical activity will be incorporated into the MEB record. The results of the MEB and an updated DA Form 3349 will then be forwarded to a PEB if the Soldier does not met medical retention standards. For RC Soldiers not on active duty, the trial of duty may consider performance in the Soldier's civilian position, as well as any military duty that may have been performed in the interim. 
c. The following profile guidelines supplement chapter 7. Individuals recommended for a trial of duty will be given a temporary P-3 profile with specific written limitations and instructions for physical and cardiovascular rehabilitation on DA Form 3349. If the Soldier later is referred to a MEB, the completed MEB will include a permanent numerical designator in the "P" factor of the physical profile that is based on functional assessment as follows:

(1) Numerical designator "1." Individuals who are asymptomatic, without objective evidence of myocardial ischemia or other cardiovascular functional abnormality (New York Heart Association Functional Class I).

(2) Numerical designator "2." Individuals with minor physical activity limitations or who require frequent medical follow-up.

(3) Numerical Designator "3." Individuals who are asymptomatic but with objective evidence of myocardial ischemia or other cardiovascular functional abnormality. Those requiring assignment limitations.

(4) Numerical designator "4." Individuals who are symptomatic (New York Heart Association Functional Class II or worse).

\section{3-26. Tuberculosis, pulmonary}

The causes for referral to an MEB for pulmonary tuberculosis:

a. If an expiration of service will occur before completion of the period of hospitalization. (Career Soldiers who express a desire to reenlist after treatment may extend their enlistment to cover the period of hospitalization.)

$b$. When a member of the USAR or ARNG/ARNGUS not on active duty has active disease that will probably require treatment for more than 12 to 15 months including an appropriate period of convalescence before he or she can perform full-time military duty. Individuals who are retained in the USAR or ARNG/ARNGUS while undergoing treatment may not be called or ordered to active duty (including mobilization), ADT, or inactive duty training (IDT) during the period of treatment and convalescence.

\section{3-27. Miscellaneous respiratory disorders}

The causes for referral to an MEB are as follows:

a. Asthma. This includes reactive airway disease, exercise-induced bronchospasm, asthmatic bronchospasm, or asthmatic bronchitis within the criteria outlined in paragraphs (1) through (4), below.

(1) Definitions/diagnostic criteria are as follows.

(a) Asthma is a clinical syndrome characterized by cough, wheeze, or dyspnea and physiologic evidence of reversible airflow obstruction or airway hyperactivity that persists over a prolonged period of time (generally more than 6 to 12 months).

(b) Reversible airflow obstruction is defined as more than 12 percent increase in forced expiratory volume in 1 second (FEVI) following the administration of an inhaled bronchodilator or prolonged corticosteroid therapy.

(c) Increased bronchial responsiveness is the presence of an exaggerated decrease in airflow induced by a standard bronchoprovocation challenge such as methacholine inhalation (PD20 FEV1 less than or equal to 4mg/ml). Demonstration of exercise induced bronchospasm (12 percent decline in FEV1) is also diagnostic of increased bronchial responsiveness; however, failure to induce bronchospasm with exercise does not rule out the diagnosis of asthma. Bronchoprovocation or exercise testing should be performed by a credentialed provider privileged to perform the procedures.

(d) Soldiers who are diagnosed as having asthma may be placed on a temporary profile under the " $\mathrm{P}$ " factor of the physical profile for up to 12 months trial of duty, when medically advisable. If at the end of that period, the Soldier is unable to perform all military training and duty as cited below, the Soldier will be referred to MEB.

(e) Acute, self limited, reversible airflow obstruction and airway hyperactivity can be caused by upper respiratory infections and inhalation of irritant gases or pollutants. This should not be permanently diagnosed as asthma unless significant symptoms or airflow abnormalities persist for more than 12 months.

(2) Chronic asthma is cause for a permanent P-3 or P-4 profile and MEB referral if it-

(a) Results in repetitive hospitalizations, repetitive emergency room visits or excessive time lost from duty.

(b) Requires repetitive use of oral corticosteroids to enable the Soldier to perform all military training and duties.

(c) Results in inability to run outdoors at a pace that meets the standards for the timed 2-mile run despite medications. (The P-3 for the inability to perform the run refers to the inability due to asthma and should not be confused with giving an L2 or L3 based on an underlying orthopedic condition that requires an alternate Army Physical Fitness Test (APFT).)

(d) Prevents the Soldier from wearing a protective mask.

(3) All Soldiers meeting an MEB for asthma should receive a consultation from an internist, pulmonologist, or allergist.

(4) Chronic asthma meets retention standards, but is a cause for a permanent P-2 profile if itbut

(a) Requires regular medications including low dose inhaled corticosteroids and/or oral or inhaled bronchodilators; 
(b) Does not prevent the Soldier from otherwise performing all military training and duties including the 2 mile run within time standards.

(5) Soldiers with a diagnosis of asthma who require no medications or activity limitations require no profiling action.

b. Atelectasis, or massive collapse of the lung. Moderately symptomatic with paroxysmal cough at frequent intervals throughout the day or with moderate emphysema or with residuals or complications that require repeated hospitalization.

c. Bronchiectasis or bronchiolectasis. Cylindrical or saccular type that is moderately symptomatic, with paroxysmal cough at frequent intervals throughout the day or with moderate emphysema with a moderate amount of bronchiectatic sputum or with recurrent pneumonia or with residuals or complications that require repeated hospitalization.

d. Bronchitis. Chronic, severe, persistent cough, with considerable expectoration or with dyspnea at rest or on slight exertion or with residuals or complications that require repeated hospitalization.

e. Cystic disease of the lung, congenital disease involving more than one lobe of a lung.

f. Diaphragm, congenital defect. Symptomatic.

g. Hemopneumothorax, hemothorax, or pyopneumothorax. More than moderate pleuritic residuals with persistent underweight or marked restriction of respiratory excursions and chest deformity or marked weakness and fatigue on slight exertion.

h. Histoplasmosis. Chronic and not responding to treatment.

$i$. Pleurisy, chronic, or pleural adhesions. Severe dyspnea or pain on mild exertion associated with definite evidence of pleural adhesions and demonstrable moderate reduction of pulmonary function.

j. Pneumothorax, spontaneous. Recurrent episodes of pneumothorax not corrected by surgery or pleural sclerosis.

k. Pneumoconiosis. Severe, with dyspnea on mild exertion.

l. Pulmonary calcification. Multiple calcifications associated with significant respiratory embarrassment or active disease not responsive to treatment.

m. Pulmonary emphysema. Marked emphysema with dyspnea on mild exertion and demonstrable moderate reduction in pulmonary function.

n. Pulmonary fibrosis. Linear fibrosis or fibrocalcific residuals of such a degree as to cause dyspnea on mild exertion and demonstrable moderate reduction in pulmonary function.

o. Pulmonary sarcoidosis. If not responding to therapy and complicated by demonstrable moderate reduction in pulmonary function.

p. Stenosis, bronchus. Severe stenosis associated with repeated attacks of bronchopulmonary infections requiring hospitalization of such frequency as to interfere with the satisfactory performance of duty.

\section{3-28. Surgery of the lungs}

The cause for referral to an MEB is a complete lobectomy, if pulmonary function (ventilatory tests) is impaired to a moderate degree or more.

\section{3-29. Mouth, esophagus, nose, pharynx, larynx, and trachea}

The causes for referral to an MEB are as follows:

a. Esophagus.

(1) Achalasia, unless controlled by medical therapy.

(2) Esophagitis, persistent and severe.

(3) Diverticulum of the esophagus of such a degree as to cause frequent regurgitation, obstruction, and weight loss that does not respond to treatment.

(4) Stricture of the esophagus of such a degree as to almost restrict diet to liquids, require frequent dilatation and hospitalization, and cause difficulty in maintaining weight and nutrition.

b. Larynx.

(1) Paralysis of the larynx characterized by bilateral vocal cord paralysis seriously interfering with speech and adequate airway.

(2) Stenosis of the larynx of a degree causing respiratory embarrassment upon more than minimal exertion.

c. Obstructive edema of glottis. If chronic, not amenable to treatment, and requires a tracheotomy.

d. Rhinitis. Atrophic rhinitis characterized by bilateral atrophy of nasal mucous membrane with severe crusting, concomitant severe headaches, and foul, fetid odor.

e. Sinusitis. Severe, chronic sinusitis that is suppurative, complicated by chronic or recurrent polyps, and that does not respond to treatment.

f. Anosmia. Permanent.

g. Trachea. Stenosis of the trachea. 


\section{3-30. Neurological disorders}

The causes for referral to an MEB are as follows:

a. Amyotrophic lateral sclerosis and all other forms of progressive neurogenic muscular atrophy.

b. All primary muscle disorders including facioscapulohumeral dystrophy, limb girdle dystrophy, and myotonic dystrophy characterized by progressive weakness and atrophy.

c. Myasthenia gravis unless clinically restricted to the extraocular muscles.

$d$. Progressive degenerative disorders of the basal ganglia and cerebellum including Parkinson's disease, Huntington's chorea, hepatolenticular degeneration, and variants of Friedreich's ataxia.

$e$. Multiple sclerosis, optic neuritis, transverse myelitis, and similar demyelinating disorders.

$f$. Stroke, including both the effects of ischemia and hemorrhage, when residuals affect performance.

g. Migraine, tension, or cluster headaches, when manifested by frequent incapacitating attacks. All such Soldiers will be referred to a neurologist, who will ascertain the cause of the headaches. If the neurologist feels a trial of prophylactic medicine is warranted, a 3-month trial of therapy can be initiated. If the headaches are not adequately controlled at the end of the 3 months, the Soldier will undergo an MEB for referral to a PEB. If the neurologist feels the Soldier is unlikely to respond to therapy, the Soldier can be referred directly to MEB.

h. Narcolepsy, sleepwalking, or similar sleep disorders. (See para 3-41.) The evaluation and treatment of these diagnoses by a neurologist or other sleep specialist should be routinely sufficient.

i. Seizure disorders and epilepsy. Seizures by themselves are not disqualifying unless they are manifestations of epilepsy. However, they may be considered along with other disabilities in judging fitness. In general, epilepsy is disqualifying unless the Soldier can be maintained free of clinical seizures of all types by nontoxic doses of medications. The following guidance applies when determining whether a Soldier will be referred to an MEB.

(1) All active duty Soldiers with suspected epilepsy must be evaluated by a neurologist who will determine whether epilepsy exists and whether the Soldier should be given a trial of therapy on active duty or referred directly to an MEB for referral to a PEB. In making the determination, the neurologist may consider the underlying cause, EEG findings, type of seizure, duration of epilepsy, Family history, Soldier's likelihood of compliance with therapeutic program, absence of substance abuse, or any other clinical factor influencing the probability of control or the Soldier's ability to perform duty during the trial of treatment.

(2) If a trial of duty on treatment is elected by the neurologist, the Soldier will be given a temporary P-3 profile with as few restrictions as possible.

(3) Once the Soldier has been seizure free for 1 year, the profile may be reduced to a P-2 profile with restrictions specifying no assignment to an area where medical treatment is not available.

(4) If seizures recur beyond 6 months after the initiation of treatment, the Soldier will be referred to an MEB.

(5) Should seizures recur during a later attempt to withdraw medications or during transient illness, referral to a PEB is at the discretion of the physician or MEB.

(6) If the Soldier has remained seizure free for 36 months, they may be removed from profile restrictions.

(7) Recurrent pseudoseizures are most commonly seen in the presence of epilepsy. As such, they do not meet the standard under the same rules as epilepsy. While each case may be individualized, their evaluation by a neurologist should be routinely sufficient.

j. Any other neurologic conditions, Traumatic Brain Injury (TBI) or other etiology, when after adequate treatment there remains residual symptoms and impairments such as persistent severe headaches, uncontrolled seizures, weakness, paralysis, or atrophy of important muscle groups, deformity, uncoordination, tremor, pain, or sensory disturbance, alteration of consciousness, speech, personality, or mental function of such a degree as to significantly interfere with performance of duty.

Note. Diagnostic concepts and terms used in paragraphs 3-31 through 3-37 are in consonance with the Diagnostic and Statistical Manual of Mental Disorders, Fourth Edition (DSM-IV). The minimum psychiatric evaluation will include Axis I, II, and III.

\section{3-31. Disorders with psychotic features}

The causes for referral to an MEB are as follows:

a. Diagnosed psychiatric conditions that fail to respond to treatment or restore the Soldier to full function within 1 year of onset of treatment.

b. Mental disorders not secondary to intoxication, infections, toxic, or other organic causes, with gross impairment in reality testing, resulting in interference with social adjustment or with duty performance.

\section{3-32. Mood disorders}

The causes for referral to an MEB are as follows:

a. Persistence or recurrence of symptoms sufficient to require extended or recurrent hospitalization; or

$b$. Persistence or recurrence of symptoms necessitating limitations of duty or duty in protected environment; or

c. Persistence or recurrence of symptoms resulting in interference with effective military performance. 


\section{3-33. Anxiety, somatoform, or dissociative disorders}

The causes for referral to an MEB are as follows:

a. Persistence or recurrence of symptoms sufficient to require extended or recurrent hospitalization; or

$b$. Persistence or recurrence of symptoms necessitating limitations of duty or duty in protected environment; or

c. Persistence or recurrence of symptoms resulting in interference with effective military performance.

\section{3-34. Dementia and other cognitive disorders due to general medical condition}

The causes for referral to an MEB include persistence of symptoms or associated personality change sufficient to interfere with the performance of duty or social adjustment.

\section{3-35. Personality, psychosexual conditions, transsexual, gender identity, exhibitionism, transvestism, voyeurism, other paraphilias, or factitious disorders; disorders of impulse control not elsewhere classified}

a. A history of, or current manifestations of, personality disorders, disorders of impulse control not elsewhere classified, transvestism, voyeurism, other paraphilias, or factitious disorders, psychosexual conditions, transsexual, gender identity disorder to include major abnormalities or defects of the genitalia such as change of sex or a current attempt to change sex, hermaphroditism, pseudohermaphroditism, or pure gonadal dysgenesis or dysfunctional residuals from surgical correction of these conditions render an individual administratively unfit.

$b$. These conditions render an individual administratively unfit rather than unfit because of physical illness or medical disability. These conditions will be dealt with through administrative channels, including AR 135-175, AR 135-178, AR 635-200, or AR 600-8-24.

\section{3-36. Adjustment disorders}

Situational maladjustments due to acute or chronic situational stress do not render an individual unfit because of physical disability, but may be the basis for administrative separation if recurrent and causing interference with military duty.

\section{3-37. Eating disorders}

The causes for referral to an MEB are eating disorders that are unresponsive to treatment or that interfere with the satisfactory performance of duty.

\section{3-38. Skin and cellular tissues}

The causes for referral to an MEB are as follows:

a. Acne. Severe, unresponsive to treatment, and interfering with the satisfactory performance of duty or wearing of the uniform or other military equipment.

b. Atopic dermatitis. More than moderate, unresponsive to treatment, and which interferes with the Soldier's performance of duty.

c. Amyloidosis. Generalized.

d. Cysts and tumors. (See paras 3-42 and 3-43.)

e. Dermatitis herpetiformis. Not responsive to therapy.

f. Dermatomyositis.

g. Dermographism. Interfering with the performance of duty.

h. Eczema, chronic. Regardless of type, when there is more than minimal involvement and the condition is unresponsive to treatment and interferes with the satisfactory performance of duty.

$i$. Elephantiasis or chronic lymphedema. Not responsive to treatment.

j. Epidermolysis bullosa.

k. Erythema multiforme. More than moderate and recurrent or chronic.

l. Exfoliative dermatitis. Chronic. duty.

m. Fungal infections, superficial. If not responsive to therapy and interfering with the satisfactory performance of

n. Hidradenitis suppurative and/or folliculitis decalvans (dissecting cellulitis of the scalp). If unresponsive to treatment and interferes with the satisfactory performance of duty.

o. Hyperhidrosis. On the hands or feet, when severe or complicated by a dermatitis or infection, either fungal or bacterial and not amenable to treatment.

p. Leukemia cutis or mycosis fungoides or cutaneous T-Cell lymphoma. (See also para 3-42.)

q. Lichen planus. Generalized and not responsive to treatment.

$r$. Lupus erythematosus. Cutaneous or mucous membranes involvement that is unresponsive to therapy and interferes with the satisfactory performance of duty.

s. Neurofibromatosis. When interfering with the satisfactory performance of duty. 
t. Panniculitis. Relapsing, febrile, nodular.

u. Parapsoriasis. Extensive and not controlled by treatment.

v. Pemphigus. Not responsive to treatment and with moderate constitutional or systemic symptoms, or interfering with the satisfactory performance of duty.

w. Psoriasis. Extensive and not controllable by treatment.

$x$. Radiodermatitis. If resulting in malignant degeneration at a site not amenable to treatment.

y. Scars and keloids. So extensive or adherent that they seriously interfere with the function of an extremity or interfere with the performance of duty.

z. Scleroderma. Generalized or of the linear type that seriously interferes with the function of an extremity.

aa. Tuberculosis of the skin. (See paragraph 3-40.)

$a b$. Ulcers of the skin. Not responsive to treatment after an appropriate period of time if interfering with the satisfactory performance of duty.

ac. Urticaria/Angioedema. Chronic, severe, and not responsive to treatment.

ad. Xanthoma. Regardless of type, but only when interfering with the satisfactory performance of duty.

ae. Intractable plantar keratosis, chronic. Requires frequent medical/surgical care or that interferes with the satisfactory performance of duty.

af. Other skin disorders. If chronic or of a nature that requires frequent medical care, or interferes with the satisfactory performance of military duty.

\section{3-39. Spine, scapulae, ribs, and sacroiliac joints}

The causes for referral to an MEB are as follows (see also para 3-14):

a. Dislocation. Congenital, of hip.

b. Spina bifida. Demonstrable signs and moderate symptoms of root or cord involvement.

c. Spondylolysis or spondylolisthesis. More than mild symptoms resulting in repeated outpatient visits, or repeated hospitalization or limitations effecting performance of duty.

d. Coxa vara. More than moderate with pain, deformity, and arthritic changes.

e. Herniation of nucleus pulposus. More than mild symptoms following appropriate treatment or remedial measures, with sufficient objective findings to demonstrate interference with the satisfactory performance of duty.

f. Kyphosis. More than moderate, interfering with military duties.

g. Scoliosis. Severe deformity with over 2 inches deviation of tips of spinous process from the midline, or of lesser degree if recurrently symptomatic and interfering with military duties.

h. Nonradicular pain involving the cervical, thoracic, lumbosacral, or coccygeal spine, whether idiopathic or secondary to degenerative disc or joint disease, that fails to respond to adequate conservative treatment and necessitates significant limitation of physical activity. Range of motion (ROM) measurements should be obtained using a goniometer (a bubble goniometer/inclometer is also acceptable). SF Form 527 should be used to document the ROM and the method of measurement. Use the VA's instructions for completion of spine and joint evaluations. This includes the six measurements shown on VASRD Plate V ROM of cervical and thoracolumbar spine.

\section{3-40. Systemic diseases}

The causes for referral to an MEB are as follows:

a. Amyloidosis.

b. Brucellosis. Chronic with substantiated, recurring febrile episodes, severe fatigue, lassitude, depression, or general malaise.

c. Leprosy. Any type that seriously interferes with performance of duty or is not completely responsive to appropriate treatment.

d. Myasthenia gravis.

e. Mycosis, Blastomycosis, Coccidioidomycosis, and Histoplasmosis. Active, not responsive to therapy or requiring prolonged treatment, or when complicated by residuals that themselves are unfitting.

f. Porphyria, cutanea tarda.

g. Sarcoidosis. Progressive with severe or multiple organ involvement and not responsive to therapy.

h. Tuberculosis.

(1) Meningitis, tuberculous.

(2) Pulmonary tuberculosis (see para 3-26), tuberculous empyema, and tuberculous pleurisy.

(3) Tuberculosis of the male genitalia. Involvement of the prostate or seminal vesicles and other instances not corrected by surgical excision, or when residuals are more than minimal, or are symptomatic.

(4) Tuberculosis of the female genitalia.

(5) Tuberculosis of the kidney.

(6) Tuberculosis of the larynx. 
(7) Tuberculosis of the lymph nodes, skin, bone, joints, eyes, intestines, and peritoneum or mesentery. These will be evaluated on an individual basis, considering the associated involvement, residuals, and complications.

i. Rheumatoid arthritis. That interferes with successful performance of duty or requires geographic assignment limitations or requires medication for control that requires frequent monitoring by a physician due to debilitating or serious side effects.

j. Spondyloarthropathies. Chronic or recurring episodes of arthritis causing functional impairment interfering with successful performance of duty supported by objective, subjective, and radiographic findings, or requires medication for control that requires frequent monitoring by a physician due to debilitating or serious side effects.

(1) Ankylosingpondylitis.

(2) Reiter's syndrome.

(3) Psoriatic arthritis.

(4) Arthritis associated with inflammatory bowel disease.

(5) Whipple's disease.

k. Systemic lupus erythematosus. That interferes with successful performance of duty or requires geographic assignment limitations or requires medication for control that requires frequent monitoring by a physician due to debilitating or serious side effects.

l. Sjogren's syndrome. When chronic, more than mildly symptomatic and resistant to treatment after a reasonable period of time.

$m$. Progressive systemic sclerosis. Diffuse and limited disease that interferes with successful performance of duty, or requires geographic assignment limitations, or requires medication for control that requires frequent monitoring by a physician due to debilitating, or serious side effects.

n. Myopathy. To include inflammatory, metabolic or inherited, that interferes with successful performance of duty or requires geographic assignment limitations or requires medication for control that requires frequent monitoring by a physician due to debilitating or serious side effects.

o. Systemic vasculitis. Involving major organ systems, chronic, that interferes with successful performance of duty, or requires geographic assignment limitations, or requires medication for control that requires frequent monitoring by a physician due to debilitating, or serious side effects.

p. Hypersensitivity angiitis. When chronic or having recurring episodes that are more than mildly symptomatic or show definite evidence of functional impairment which is resistant to treatment after a reasonable period of time.

q. Behcet's syndrome. That interferes with successful performance of duty or requires geographic assignment limitations or requires medication for control that requires frequent monitoring by a physician due to debilitating or serious side effects.

$r$. Adult onset Still's disease. That interferes with successful performance of duty or requires geographic assignment limitations or requires medication for control that requires frequent monitoring by a physician due to debilitating or serious side effects.

t. Mixed connective tissue disease and other overlap syndromes. That interfere with successful performance of duty or require geographic assignment limitations or require medication for control that requires frequent monitoring by a physician due to debilitating or serious side effects.

$u$. Exertional rhabdomyolysis. The diagnosis of exertional rhabdomyolysis, defined as severe exercise-induced muscle pain resulting from repetitive exercise with an elevation of serum creatine kinase (CK) generally at least 5 times the upper limit of the lab normal range or urine myoglobin, will be referred to a MEB if the Soldier has-

(1) Recurrent episodes of exertional rhabdomyolysis; or

(2) A single episode with severe systemic complications (for example, compartment syndrome); or

(3) A single episode results in physical complications that interfere with successful performance of duty.

(4) Soldiers with any of the following symptoms 2 weeks after experiencing an episode of exertional rhabdomyolyis should be referred to the appropriate specialist for consideration of referral to an MEB:

(a) Persistent residual kidney injury; or

(b) Persistent elevation of serum CK 5 times the upper limit of the lab normal range or delayed clinical recovery; or

(c) A history of sickle cell trait.

(5) The Uniformed Services University Consortium for Health and Military Performance (CHAMP) (http://champ. usuhs.mil) is available electronically to assist in clinical consultation at champ@usuhs.mil. In addition, a Clinical Practice Guideline in the Management of Exertional Rhabdomyolysis in Soldiers is available at: http://champ.usuhs.mil/ chclinicaltools.html.

v. Any chronic or recurrent systemic inflammatory disease or arthritis not listed above. That interferes with successful performance of duty or requires geographic assignment limitations, or requires medication for control that requires frequent monitoring by a physician due to debilitating or serious side effects.

\section{3-41. General and miscellaneous conditions and defects}

The causes for referral to an MEB are as follows: 
a. Allergic manifestations.

(1) Allergic rhinitis, chronic, severe, and not responsive to treatment. (See also paras 3-29d and 3-29e.)

(2) Asthma. (See para 3-27a.)

(3) Allergic dermatoses. (See para 3-38.)

b. Cold injury/heat injury. (See paras 3-45 and 3-46.)

c. Sleep apnea. Obstructive sleep apnea or sleep-disordered breathing that causes daytime hypersomnolence or snoring that interferes with the sleep of others and that cannot be corrected with medical therapy, nasal continuous positive airway pressure (CPAP), surgery, or an oral appliance. The diagnosis must be based upon a nocturnal polysomnogram and the evaluation of a pulmonologist, neurologist, or a privileged provider with expertise in sleep medicine.

(1) A 12-month trial of therapy with nasal continuous positive air pressure may be attempted to assist with other therapeutic interventions, during which time the individual will be issued a temporary profile. Soldiers with severe sleep apnea and/or symptoms may be referred directly for an MEB. If nasal CPAP is required for longer than 12 months, the Soldiers should be profiled as a permanent P2.

(2) If symptoms of hypersomnolence or snoring can not be controlled with medical therapy, nasal CPAP, surgery or an oral appliance, the individual should be referred for a MEB. If the use of nasal CPAP or other therapies for sleep apneas result in interference with satisfactory performance of duty as substantiated by the individual's commander or supervisor, the Soldier should be referred to a MEB.

d. Fibromyalgia. When severe enough to prevent successful performance of duty. Diagnosis will include evaluation by a rheumatologist.

e. Miscellaneous conditions and defects. Conditions and defects not mentioned elsewhere in this chapter are causes for referral to an MEB, if-

(1) The conditions (individually or in combination) result in interference with satisfactory performance of duty as substantiated by the individual's commander or supervisor. Any medical condition, injury or defect (individually or in combination) that prevents the Soldier from performing any of the functional activities listed under item number 5 on DA Form 3349 (Physical Profile).

(2) The individual's health or well-being would be compromised if they were to remain in the military service.

(3) In view of the Soldier's condition, their retention in the military service would prejudice the best interests of the Government (for example, a carrier of communicable disease who poses a health threat to others). Questionable cases, including those involving latent impairment, will be referred to PEBs.

\section{3-42. Malignant neoplasms}

The causes for referral to an MEB are as follows:

a. Malignant neoplasms that are unresponsive to therapy, or when the residuals of treatment are in themselves unfitting under other provisions of this chapter.

$b$. Neoplastic conditions of the lymphoid and blood-forming tissues that are unresponsive to therapy, or when the residuals of treatment are in themselves unfitting under other provisions of this chapter.

c. Malignant neoplasms, when on evaluation for administrative separation or retirement, the observation period subsequent to treatment is deemed inadequate in accordance with accepted medical principles.

$d$. The above definitions of malignancy or malignant disease exclude basal cell carcinoma of the skin.

\section{3-43. Benign neoplasms}

The causes for referral to an MEB are as follows:

a. Benign tumors if their condition precludes the satisfactory performance of military duty.

b. Ganglioneuroma.

c. Meningeal fibroblastoma, when the brain is involved.

$d$. Pigmented villonodular synovitis when severe enough to prevent successful performance of duty.

\section{3-44. Sexually transmitted diseases}

The causes for referral to an MEB are as follows:

a. Symptomatic neurosyphilis in any form.

b. Complications or residuals of a sexually transmitted disease of such chronicity or degree that the individual is incapable of performing useful duty.

\section{3-45. Exertional heat illness}

General. Exertional heat illness represents a continuum in severity, and includes heat exhaustion, heat injury, and heat stroke. Heat stroke should be the working diagnosis for any Soldier with profound altered mental status. Final diagnosis should be delayed until the entire clinical picture is evident. The causes for referral to an MEB are as follows: 
a. Heat exhaustion (HE) is defined as a syndrome of hyperthermia (core temperature at time of event usually $\leq 40^{\circ} \mathrm{C}$ or $104^{\circ} \mathrm{F}$ ) with physical collapse or debilitation occurring during or immediately following exertion in the heat, with no more than minor central nervous system (CNS) dysfunction (such as headache, dizziness). HE resolves rapidly with minimal cooling intervention.

(1) Individual episodes of HE are not cause for referral to a MEB. However, Soldiers who experience three episodes of $\mathrm{HE}$ in less than 24 months, require referral to an MEB.

(2) Soldiers diagnosed with HE are individually profiled as determined by the treating privileged provider. Soldiers with HE pending referral to a MEB will be profiled using guidance provided in table 3-2.

$b$. Heat injury (HI) is defined as HE with clinical evidence of organ (for example, liver, renal, stomach) and/or muscle (for example, rhabdomyolysis) damage without sufficient neurological symptoms to be diagnosed as heat stroke.

(1) Single episodes of HI are not cause for an immediate referral to a MEB. However, Soldiers who experience three episodes of $\mathrm{HI}$ in less than 24 months or a single episode with severe complications (for example, compartment syndrome) of such a nature that the complications interfere with successful performance of duty, require referral to a MEB. Soldiers demonstrating any of the following complications, despite two weeks of rest, should be referred to the appropriate medical specialist for consideration of referral to a MEB: persistent residual kidney injury; persistent elevation of serum creatine kinase (CK) more than 5 times the upper limit of the lab normal range or persistent elevation of transaminases more than three times the upper limit of the lab normal range.

(2) All Soldiers diagnosed with HI will be placed on a temporary profile, numerical designator 4 in the PULHES physical capacity factor P, (T4-(P)), for a period of 1 week. After the 1-week period, the Soldier will be reevaluated and individually profiled as determined by the treating privileged provider. Soldiers diagnosed with $\mathrm{HI}$ and pending referral to a MEB will be profiled using guidance provided in table 3-2.

c. Heat stroke (HS) is defined as a syndrome of hyperthermia (core temperature at time of event usually $\geq 40^{\circ} \mathrm{C}$ or $104^{\circ} \mathrm{F}$ ), physical collapse or debilitation, and encephalopathy as evidenced by delirium, stupor, or coma, occurring during or immediately following exertion or significant heat exposure. The HS can be complicated by organ and/or tissue damage, systemic inflammatory activation, and disseminated intravascular coagulation.

(1) Following an episode of HS, the Soldier will be placed on a T4-(P) profile for a period of 2 weeks. After the 2week period, the Soldier will be reevaluated weekly for the need of a continuing profile and/or referral to a MEB. This reevaluation will include an assessment for the presence or absence of physical damage and/or complications and any contributing risk factor(s) that may have increased the Soldier's inability to tolerate the heat exposure. For profile guidance, see table $3-2$.

(2) During the reevaluation period, the Soldier will be classified into one of the following three categories: HS without sequelae demonstrated by all clinical signs and symptoms resolved by 2 weeks following the heat exposure event; HS with sequelae to include any evidence of cognitive or behavioral dysfunction, renal impairment, hepatic dysfunction, rhabdomyolysis, or other related pathology that does not completely resolve by 2 weeks following the heat exposure event; or complex HS that is recurrent, or occurring in the presence of a non-modifiable risk factor, either known (for example, a chronic skin condition such as eczema or burn skin graft) or suspected (for example, sickle cell trait or malignant hyperthermia susceptibility).

(3) Soldiers with complex HS require referral to a MEB. The Soldier's provider should consider referring the Soldier to a center with clinical expertise in heat illness for further evaluation.

$d$. Initial entry training Soldiers will not be separated based upon the diagnosis of one episode of HS with or without complications, but will be placed into a Warrior Training and Rehabilitation Program (WTRP) (formally the Physical Training and Rehabilitation Program (PTRR)) for the duration of their profile.

$e$. The Uniformed Services University Consortium for Health and Military Performance (CHAMP) (http://champ. usuhs.mil) is available electronically to assist in clinical consultation at champ@usuhs.mil. The U.S. Army Research Institute of Environmental Medicine (USARIEM) (www.usariem.army.mil ) and U.S. Army Center for Health Promotion and Preventive Medicine (USACHPPM) (http://chppm-www.apgea.army.mil/) are subject matter experts in heat physiology, acclimation and related operational issues, and offer valuable clinical and educational resources.

\section{3-46. Cold injury}

The causes for referral to an MEB are as follows:

a. Frostbite (freezing cold injury).

(1) The definition of frostbite is the consequence of freezing of tissue. First degree frostbite is manifested by superficial injury without blistering. Second degree frostbite is manifested by superficial injury with clear blisters with only epidermal tissue loss. Third degree and fourth degree frostbite are manifested by significant subepidermal tissue loss.

(2) Soldiers with first degree frostbite after clinical healing will be given a permanent $\mathrm{P}-2$ profile permitting the use of extra cold weather protective clothing, including nonregulation items, to be worn under authorized outer garments.

(3) Soldiers with frostbite more than first degree will be given a P-3 profile, renewed as appropriate, for the duration of the cold season restricting them from any exposure to temperatures below 0 degrees $\mathrm{C}$ ( 32 degrees F) and 
from any activities limited by the remainder of the season. After the cold season, Soldiers will be reevaluated and, if appropriate, given the $\mathrm{P}-2$ profile described in (2), above.

(4) Soldiers will be referred to an MEB for recurrent cold injury, recurrent or persistent cold sensitivity despite the P-2 profile, vascular or neuropathic symptoms, or disability due to tissue lost from cold injury.

b. Trench foot (nonfreezing cold injury).

(1) The definition of trench foot is the consequence of prolonged cold immersion of an extremity. It is manifested by maceration of tissue and neurovascular injury.

(2) Soldiers with residual symptoms or significant tissue loss after healing will be referred to an MEB.

c. Accidental hypothermia.

(1) The definition of accidental hypothermia is clinically significant depression of body temperature due to environmental cold exposure.

(2) Soldiers with significant symptoms of cold intolerance or a recurrence of hypothermia after an episode of accidental hypothermia will be referred to an MEB.

\begin{tabular}{|c|c|c|c|c|}
\hline Class & $\begin{array}{l}\text { New York Heart Association } \\
\text { Functional Classification }\end{array}$ & $\begin{array}{l}\text { Canadian Cardiovascular Soci- } \\
\text { ety Functional Classification }\end{array}$ & $\begin{array}{l}\text { Specific activity scale (Goldstein } \\
\text { et al: Circulation } 64: 1227,1981 \text { ) }\end{array}$ & $\begin{array}{l}\text { New York Heart Association } \\
\text { Functional Classification (Re- } \\
\text { vised) }\end{array}$ \\
\hline I. & $\begin{array}{l}\text { Patient with cardiac disease } \\
\text { but without resulting limita- } \\
\text { tions of physical activity. Or- } \\
\text { dinary physical activity does } \\
\text { not cause undue fatigue, } \\
\text { palpitations, dyspnea, or an- } \\
\text { ginal pain. }\end{array}$ & $\begin{array}{l}\text { Ordinary physical activity, } \\
\text { such as walking and climb- } \\
\text { ing, stairs, does not cause } \\
\text { angina. Angina with strenu- } \\
\text { ous or rapid or prolonged } \\
\text { exertion at work or recrea- } \\
\text { tion. }\end{array}$ & $\begin{array}{l}\text { Patients can perform to } \\
\text { completion any activity re- } \\
\text { quiring } 7 \text { metabolic equiva- } \\
\text { lents: for example, can carry } \\
24 \text { lbs up eight steps, carry } \\
\text { objects that weigh } 80 \text { lbs, } \\
\text { do outdoor work. (shovel } \\
\text { snow, spade soil), do } \\
\text { recreational activities (ski- } \\
\text { ing, basketball, handball, } \\
\text { jog, and walk } 5 \mathrm{mph} \text { ). }\end{array}$ & $\begin{array}{l}\text { Cardiac status uncom- } \\
\text { promised. }\end{array}$ \\
\hline II. & $\begin{array}{l}\text { Patients with cardiac dis- } \\
\text { ease resulting in slight limi- } \\
\text { tation of physical activity. } \\
\text { They are comfortable at } \\
\text { rest. Ordinary physical ac- } \\
\text { tivity results in fatigue, pal- } \\
\text { pitation, dyspnea, or anginal } \\
\text { pain }\end{array}$ & $\begin{array}{l}\text { Slight limitations of ordinary } \\
\text { activity. Walking or climbing } \\
\text { stairs rapidly, walking uphill, } \\
\text { walking or stair climbing af- } \\
\text { ter meals, in cold, in wind, } \\
\text { or when under emotional } \\
\text { stress, or only during the } \\
\text { few hours after awakening. } \\
\text { Walking more than } 2 \text { blocks } \\
\text { on the level and climbing } \\
\text { more than one flight of ordi- } \\
\text { nary stairs at a normal pace } \\
\text { and in normal conditions. }\end{array}$ & $\begin{array}{l}\text { Patient can perform to com- } \\
\text { pletion any activity requiring } \\
\geq 5 \text { metabolic equivalents, } \\
\text { but cannot and does not } \\
\text { perform to completion activi- } \\
\text { ties requiring metabolic } \\
\text { equivalents: for example, } \\
\text { have sexual intercourse } \\
\text { without stopping, garden, } \\
\text { rake, weed, roller skate, } \\
\text { dance fox trot, walk at } 4 \\
\text { mph on level ground. }\end{array}$ & Slightly compromised. \\
\hline III. & $\begin{array}{l}\text { Patients with cardiac dis- } \\
\text { ease resulting in marked } \\
\text { limitation of physical activity. } \\
\text { They are comfortable at } \\
\text { rest. Less than ordinary } \\
\text { physical activity causes fa- } \\
\text { tigue, palpitation, dyspnea, } \\
\text { or anginal pain. }\end{array}$ & $\begin{array}{l}\text { Marked limitation of ordinary } \\
\text { physical activity. Walking } \\
\text { one to two blocks on the } \\
\text { level and climbing more } \\
\text { than one flight in normal } \\
\text { conditions. }\end{array}$ & $\begin{array}{l}\text { Patient can perform to com- } \\
\text { pletion any activity requiring } \\
\geq 2 \text { metabolic equivalents } \\
\text { but cannot and does not } \\
\text { perform to completion activi- } \\
\text { ties requiring } \geq 5 \text { metabolic } \\
\text { equivalents: for example, } \\
\text { shower without stopping, } \\
\text { strip and make bed, clean } \\
\text { windows, walk } 2.5 \mathrm{mph} \text {, } \\
\text { bowl, play golf, dress with- } \\
\text { out stopping. }\end{array}$ & Moderately compromised. \\
\hline IV. & $\begin{array}{l}\text { Patient with cardiac disease } \\
\text { resulting in inability to carry } \\
\text { on any physical activity } \\
\text { without discomfort. Symp- } \\
\text { toms of cardiac insufficiency } \\
\text { or of the anginal syndrome } \\
\text { may be present even at } \\
\text { rest. If any physical activity } \\
\text { is undertaken, discomfort is } \\
\text { increased. }\end{array}$ & $\begin{array}{l}\text { Inability to carry on any } \\
\text { physical activity without dis- } \\
\text { comfort-anginal syndrome } \\
\text { may be present at rest. }\end{array}$ & $\begin{array}{l}\text { Patient cannot or does not } \\
\text { perform to completion activi- } \\
\text { ties requiring } \geq 2 \text { metabolic } \\
\text { equivalents. Cannot carry } \\
\text { activities listed above (spec- } \\
\text { ify activity scale, Class III). }\end{array}$ & Severely compromised. \\
\hline
\end{tabular}


Table 3-1

Methods of assessing cardiovascular disability-Continued

\begin{tabular}{l|l|l|l|l}
\hline Class & $\begin{array}{l}\text { New York Heart Association } \\
\text { Functional Classification }\end{array}$ & $\begin{array}{l}\text { Canadian Cardiovascular Soci- } \\
\text { ety Functional Classification }\end{array}$ & $\begin{array}{l}\text { Specific activity scale (Goldstein } \\
\text { et al: Circulation 64:1227, 1981) }\end{array}$ & $\begin{array}{l}\text { New York Heart Association } \\
\text { Functional Classification (Re- } \\
\text { vised) }\end{array}$ \\
\hline \multicolumn{5}{|c|}{ New York Heart Association Therapeutic Classification } \\
\hline Therapeutic Classification & & $\begin{array}{l}\text { Revised classification (prog- } \\
\text { nosis) }\end{array}$ \\
\hline Class A- & Patients with cardiac disease whose physical activity need not be restricted & Class I-Good. \\
\hline Class B- & $\begin{array}{l}\text { Patients with cardiac disease whose ordinary activity need not be restricted, but who } \\
\text { should be advised against severe or competitive physical efforts. }\end{array}$ & Class II-Good with therapy. \\
\hline Class C- & $\begin{array}{l}\text { Patients with cardiac disease whose ordinary physical activity should be moderately re- } \\
\text { stricted, and whose more strenuous efforts should be discontinued. }\end{array}$ & Class III-Fair with therapy. \\
\hline Class D- & Patients with cardiac disease who should be at complete rest, confined to bed or chair. & $\begin{array}{l}\text { Class IV-Guarded despite } \\
\text { therapy. }\end{array}$ \\
\hline
\end{tabular}

METS Equivalents (Required for PEB adjudication)

Class $\mathrm{I}=8$ METS or greater

Class $\|=5-8$ METS

Class III $=3-5$ METS

Class IV=Less than 3 METS

\begin{tabular}{|c|c|c|c|c|}
\hline Profile code ${ }^{*}$ & Restrictions ${ }^{\star *}$ & $\begin{array}{l}\text { Heat stroke without Sequ- } \\
\text { elae }\end{array}$ & Heat stroke with Sequelae & $\begin{array}{l}\text { Complex heat stroke or heat } \\
\text { exhaustion/heat injury pending } \\
\text { MEB }\end{array}$ \\
\hline T-4 (P) & Complete duty restrictions. & 2 weeks & $\begin{array}{l}2 \text { week minimum, ad- } \\
\text { vance when clinically re- } \\
\text { solved. }\end{array}$ & $\begin{array}{l}2 \text { week minimum, advance } \\
\text { when clinically resolved. }\end{array}$ \\
\hline T-3 (P) & $\begin{array}{l}\text { Physical Training and running/walking/ } \\
\text { swimming/bicycling at own pace and } \\
\text { distance not to exceed } 60 \text { min per day. } \\
\text { No maximal effort; no APFT; no wear } \\
\text { of IBA; no MOPP gear; no ruck march- } \\
\text { ing. No airborne operations (AO). }\end{array}$ & 1 month minimum & 2 months minimum & Pending MEB \\
\hline T-3 (P) & $\begin{array}{l}\text { Gradual acclimatization (TB Med 507). } \\
\text { No maximal effort; no APFT; no MOPP } \\
\text { IV gear. IBA limited to static range par- } \\
\text { ticipation. May ruck march at own pace } \\
\text { and distance with no more than } 30 \text { lbs. } \\
\text { Non tactical AO permitted. }\end{array}$ & 1 month minimum & 2 months minimum ${ }^{* * *}$ & $\mathrm{~N} / \mathrm{A}$ \\
\hline T-2 (P) & $\begin{array}{l}\text { Continue gradual acclimatization. May } \\
\text { participate in unit PT; CBRN training } \\
\text { with MOPP gear for up to } 30 \text { min; IBA } \\
\text { on static and dynamic ranges for up to } \\
45 \text { min; no record APFT. Ruck march } \\
\text { at own pace and distance with no more } \\
\text { than } 30 \text { lbs up to } 2 \text { hrs. Non-tactical } \\
\text { AO permitted. }\end{array}$ & N/A & $\begin{array}{l}\text { Pending completion of } 30 \\
\text { day heat exposure re- } \\
\text { quirement, if not accom- } \\
\text { plished during prior pro- } \\
\text { file }\end{array}$ & $N / A$ \\
\hline
\end{tabular}

*Temporary Profile; Physical Category P (PULHES).

** Soldiers manifesting no heat illness symptomatology or work intolerance after completion of profile restrictions can advance and return to duty without a MEB. Any evidence/manifestation of heat illness symptomatology during the period of the profile requires a MEB referral.

${ }^{* * *} \mathrm{HS}$ with Sequelae return to full duty requires a minimum period of heat exposure during environmental stress (Heat Category 2 during the majority of included days). 


\section{Chapter 4 \\ Medical Fitness Standards for Flying Duty}

\section{4-1. General}

a. In this regulation, the term "flying duty" is synonymous with "flight status" and "aviation service." The term "aircrew" or "aircrew member" applies to rated and non-rated personnel in aviation service, unmanned aerial systems (UAS), and air traffic control. All provisions apply to the AA, USAR, and the ARNG/ARNGUS.

$b$. The aviation medicine consultant (AMC) to TSG will recommend to TSG a senior specialist in aerospace medicine to be placed on orders for designation as the Director, U.S. Army Aeromedical Activity (USAAMA). Responsibilities will include all administrative actions and medical fitness standards for flying duty for all active and RC/NG Army aviators. The U.S. Army Aeromedical Activity is located at Building 301, Dustoff Street, Fort Rucker, AL 36362-5333.

c. Provisions in this chapter are subject to NATO Standardization Agreement (STANAG) 3526, which applies to allied nation aircrews serving with U.S. Forces or attending U.S. Army training programs, and to U.S. aircrews serving with foreign forces (see Aeromedical Technical Bulletins (ATB), International Military Students for additional guidance at: https://aamaweb.usaama.rucker.amedd.army.mil/).

$d$. This chapter lists medical conditions and physical defects that are causes for rejection in selection, training, and retention of-

(1) Army aviators.

(2) DA civilian (DAC) pilots and contract civilian pilots who are employed by firms under contract to DA.

(3) Flight surgeons (FSs) (AOC 61N), aeromedical physician assistants (APAs) (AOC 65 DM3), and aviation medicine nurse practitioners (AMNP) (AOC 66NP1). Aviation medical examiners (AME defined in para 6-9f) and DAC/contract civilian FS/APA/AMNP are not required to meet these standards.

(4) Military, DAC, and DA contract air traffic controllers (ATCs).

(5) Individuals ordered by competent authority to participate in regular flights as nonrated aircrew and UAS operators.

(6) Applicants for special flight training programs directed by DA or National Guard Bureau (NGB), such as Army ROTC or USMA flight training programs.

(7) Aircrew of allied host nations or U.S. Government agencies other than DA who are flying Army aircraft, unless superseded by agreements with that nation or agency.

$e$. A failure to meet medical standards for flying duties remains disqualifying for flying duties until reviewed by the USAAMA. The USAAMA may recommend qualified, qualified with waiver, or medical suspension from aviation service. The USAAMA issues Aeromedical Policy Letters (APLs) and ATBs that provide detailed recommendations for specific, common disqualifications. Refer all questionable cases to the USAAMA, Fort Rucker, AL 36362-5333.

\section{4-2. Classes of medical standards for flying and applicability}

The classes of medical fitness standards for flying duties are as follows:

a. Class 1 (warrant officer candidate, commissioned officer or cadet) standards apply to-

(1) Applicants for aviator training. (See also AR 611-85 and AR 611-110.)

(2) Applicants for special flight training programs directed by DA or NGB, such as Army ROTC or USMA flight training programs.

(3) Other non-U.S. Army personnel selected for training until the beginning of training at aircraft controls, or as determined by Chief, Army Aviation Branch.

$b$. Class 2 standards apply to-

(1) Student aviators after beginning training at aircraft controls or as determined by Chief, Army Aviation Branch.

(2) Rated Army aviators (AR 600-105).

(3) The DAC pilots.

(4) Contractor pilots will have the option, as specified in the contract, of maintaining either a current annual Federal Aviation Administration (FAA) Class 2 Medical Certificate or an Army Class 2 Flying Duty Medical Examination (FDME). Army Aeromedical Surveillance is an integral part of Army Aviation Risk Management. Therefore, contractor aircrew who opt for the annual FAA certificates must submit a copy of the annual FAA certificate, with any applicable Statement of Demonstrated Ability (SODA) or FAA waiver, to USAAMA and give permission to the FAA to provide their medical information to the U.S. Army Aeromedical Activity in order to continue population-based medical surveillance and ensure risks to flight safety are minimized. The aforementioned information will be mailed to USAAMA (MCXY-AER), Building 301, Dustoff Street, Fort Rucker, AL 36362; or faxed to commercial 334-2557030 ext. 7060 (DSN 558); or scanned and e-mailed to aama@amedd.army.mil.

(5) Army aviators considered for return to aviation Service.

(6) When directed by DA or NGB under special procurement programs for initial Army aviation flight training, selected senior career officers of the Army may be medically qualified under Army Class 2 medical standards. 
(7) Applicants to DA or NGB civilian-acquired aeronautical skills programs.

(8) Other non-U.S. Army personnel.

c. Class $2 \mathrm{~F} / 2 \mathrm{P}$ standards apply to-

(1) The FSs (AR 600-105); APAs and AMNPs (AR 600-106).

(2) Medical officers, medical students, nurse practitioners, and physician assistants applying for or enrolled in the Army Flight Surgeon Primary Course.

$d$. Class 3 standards apply to non-rated crewmembers (AR 600-106). Soldiers and civilians ordered by a competent authority to participate in regular flights in Army aircraft, but who do not operate aircraft flight controls. These include crew chiefs, aviation maintenance technicians, aerial observers, gunners; unmanned aircraft system operators (UASO), nonrated (AR 600-106) medical personnel selected for aeromedical training, such as flight medical aidmen, psychologists, and others (see para 4-32). Army civilian contractor non-rated crewmembers will have the option, similar to paragraph 4-2b(4), of maintaining either an annual FAA Class 3 Medical Certificate or DA Form 4186, Medical Recommendation for Flying Duty.

e. Class 4 standards apply to military ATCs. Civilian ATCs are required to meet Class IV OPM standards (see ATB, ATC Civilian Standards, DAC, and contract).

\section{4-3. Aeromedical consultation}

Aeromedical administration is detailed in chapter 6. Questions pertaining to aeromedical consultation, policy, standards, and administration should be directed to the USAAMA, Fort Rucker, AL 36362-5333.

\section{4-4. Abdomen and gastrointestinal system}

The causes for medical unfitness for flying duty Classes $1 / 2 / 2 F / 3 / 4$ are the causes listed in paragraph $2-3$, plus the following:

a. Abdominal fistula or sinus.

b. Small and large intestine.

(1) History of bowel resection for any cause, with the exception of appendectomy.

(2) History of any procedures for the relief of intestinal obstruction, adhesions, or intussusception, with the exception of uncomplicated pylorotomy or intussusception in childhood.

(3) History of functional bowel syndrome (irritable colon), megacolon, diverticulitis, diverticulosis with complications, regional enteritis (Crohn's disease), ulcerative colitis, or proctitis.

c. Hepato-pancreato-biliary tract.

(1) Enlargement of the liver, except when the liver function tests are normal and the condition does not appear to be caused by active disease.

(2) Cholelithiasis.

(3) Cholecystectomy until recovery is complete or history of sequelae to cholecystectomy listed in paragraph 2-3.

d. History of gastrointestinal bleeding. This excludes minor bleeding from hemorrhoids or acute rectal fissure. (See APLs, Peptic Ulcer Disease.)

\section{4-5. Blood and blood-forming tissue diseases}

The causes of medical unfitness for flying duty Classes $1 / 2 / 2 \mathrm{~F} / 3 / 4$ are the causes in paragraph $2-4$, plus the following:

a. Anemia, of any etiology.

(1) Males with a hematocrit (HCT) less than 40 percent, or females with an HCT less than 37 percent; or

(2) If a complete hematologic evaluation results in the diagnosis of physiologic anemia, or anemia due to sickle cell trait or beta thalassemia minor; males with a HCT less than 38 percent, or females with a HCT less than 35 percent. (See APL, Hematocrit, and Hemoglobinopathies.)

b. History of immunodeficiency diseases. (See also para 2-35l.) Civilian employees are not disqualified based solely on the presence of the HIV virus. (See AR 600-110 and ATB 2, Army Flight Surgeon's Administrative Guide.)

c. History of splenectomy. For any reason, except trauma.

d. Thrombophlebitis.

(1) Acute, superficial thrombophlebitis until resolved.

(2) History of deep vein thrombophlebitis, thrombosis of any deep vessel, or thromboembolism.

\section{4-6. Dental}

The causes of medical unfitness for flying duty Classes $1 / 2 / 2 \mathrm{~F} / 3 / 4$ are the causes in paragraph $2-5$, plus the following: $a$. Orthodontic appliances, if they interfere with effective oral communication, or pose a hazard to personal or flight safety.

b. Dental Fitness Class 3 or 4, until the abnormalities or deficiencies have been corrected (see APL, Dental Fitness). 


\section{4-7. Ears}

The causes of medical unfitness for flying duty Classes $1 / 2 / 2 \mathrm{~F} / 3 / 4$ are the causes in paragraph $2-6$, plus the following:

a. Infection. Any infectious process of the ear until completely healed, except mild asymptomatic external otitis.

b. External ear.

(1) Deformities of the pinna that cause distractions or hearing loss while wearing protective headgear.

(2) History of post auricular fistula.

c. Middle ear.

(1) Barotitis media, until resolved.

(2) History of cholesteatoma.

(3) History of chronic or recurrent Eustachian tube dysfunction.

(4) Otosclerosis.

(5) History of simple, radical, or modified radical mastoidectomy.

(6) Any surgical procedure in the middle ear that includes fenestration of the oval window or horizontal semicircular canal, any endolymphatic shunting procedure, stapedectomy, the use of any prosthesis or graft, or reconstruction of the stapes.

(7) Tympanoplasty, until completely healed with acceptable hearing and motility, as documented by current ear-nose-throat evaluation.

d. Inner ear.

(1) Abnormal labyrinthine function.

(2) History of perilymph fistula.

(3) Tinnitus, except when associated with high frequency hearing loss.

(4) History of vertigo, except physiologic vertigo induced by gravity forces, aircraft spins, or Baranay chair.

\section{4-8. Hearing}

The causes of medical unfitness for flying duty Classes $1 / 2 / 2 \mathrm{~F} / 3 / 4$ is hearing loss in $\mathrm{dB}$ greater than shown in table 4-1. (See APL, Audiometric Evaluation.)

\section{4-9. Endocrine and metabolic diseases}

The causes of medical unfitness for flying duty Classes $1 / 2 / 2 \mathrm{~F} / 3 / 4$ are the causes listed in paragraph $2-8$, plus a history of symptomatic hypoglycemia. (See APL, Diabetes and Glucose Intolerance.)

\section{4-10. Extremities}

The causes of medical unfitness for flying duty Classes $1 / 2 / 2 \mathrm{~F} / 3 / 4$ are the causes in paragraphs $2-9,2-10,2-11$, and 4-22, plus dimensions, loss of strength or endurance, or limitation in motion that compromises flying safety. Orthopedic hardware is disqualifying until reviewed by the USAAMA. (See APL, Retained Hardware.)

\section{4-11. Eyes}

The causes of medical unfitness for flying duty Classes $1 / 2 / 2 \mathrm{~F} / 3 / 4$ are the causes in paragraph $2-12$, plus the following:

a. Lids and conjunctiva.

(1) Epiphora (chronic tearing).

(2) Trachoma, unless healed without cicatrices.

b. Cornea.

(1) Complications secondary to use of contact lenses or a history of orthokeratologic procedures to correct refractive error may be disqualifying. Contact lens use requires annual followup. (See APL, Contact Lens Wear.)

(2) History of herpetic corneal ulcer or keratitis-acute, chronic, or recurrent.

(3) Pterygium that encroaches on the cornea more than $1 \mathrm{~mm}$ or is progressive, or for Classes 1, history of surgical removal of a pterygium within the last 12 months.

c. History of ocular surgery to include refractive surgery and/or interocular lens implant. (See APL, Corneal Refractive Surgery.)

d. Uveal tract.

(1) Coloboma of the choroid or iris.

(2) History of inflammation of the uveal tract, acute, chronic, or recurrent; including anterior uveitis, peripheral uveitis or pars planitis, posteri or uveitis, or traumatic iritis.

e. Retina.

(1) History of central serous retinopathy.

(2) History of chorioretinitis, including evidence of presumed ocular histoplasmosis syndrome.

(3) History of retinal holes or tears. 


\section{f. Optic nerve.}

(1) Optic nerve drusen or hyaline bodies of the optic nerve.

(2) History of optic or retrobulbar neuritis.

g. Ocular motility.

(1) History of extraocular muscle surgery after age 4, or history of extraocular muscle surgery before age 4 with other residual ocular abnormalities.

(2) Monofixation syndrome (microtropias).

h. Miscellaneous defects and diseases.

(1) Glaucoma as evidenced by applanation tension $30 \mathrm{mmHg}$ or higher, or secondary changes in the optic disc or visual field associated with glaucoma. (See APL, Glaucoma and Ocular Hypertension.)

(2) Intraocular hypertension as evidenced by two or more determinations of $22 \mathrm{mmHg}$ or higher, or a persistent difference of 4 or more mmHg tension between the two eyes, when confirmed by applanation tonometry. (See APL, Glaucoma and Ocular Hypertension.)

(3) History of penetrating trauma to the eye or hyphema.

(4) History of ocular or acephalic migraine with visual disturbance.

\section{4-12. Vision}

The causes of medical unfitness for flying duty Classes 1/2/2F/3/4 are the following:

a. Class 1. Any disqualifying condition must be referred to optometry or ophthalmology for verification.

(1) Distant visual acuity. Uncorrected distant visual acuity worse than 20/50 in each eye. If the distant visual acuity is 20/50 or better in either eye, each eye must be correctable to 20/20 with no more than 1 error per 5 presentations of 20/20 letters, in any combination, on either the Armed Forces Vision Tester (AFVT) or any projected Snellen chart set at 20 feet. (See ATB, Distant Visual Acuity Testing and APL, Decreased Visual Acuity.)

(2) Near visual acuity. Uncorrected near visual acuity worse than 20/20 in each eye; with no more than 1 error per 5 presentations of 20/20 letters, in any combination, on the AFVT or any Snellen near visual acuity card. (See ATB, Near Visual Acuity Testing and APL, Decreased Visual Acuity.)

(3) Cycloplegic refractive error using the method in ATB, Cycloplegic Refraction.

(a) Hyperopia greater than +3.00 diopters of sphere in any meridian by transposition in either eye. (Spherical equivalent method does not apply.)

(b) Myopia greater than -1.50 diopters of sphere in any meridian by transposition in either eye. (Spherical equivalent method does not apply.)

(c) Astigmatism greater than $+/-1.00$ diopter of cylinder in either eye.

(4) Ocular motility. (See ATB, Ocular Motility Testing; APL, Excessive Phorias; and APL, Convergence Insufficiency.)

(a) Any degree of tropia detected in ocular motion on the Cover-Uncover Test (Unilateral Cover Test or Tropia Test).

(b) Esophoria greater than 8 prism diopters.

(c) Exophoria greater than 8 prism diopters.

(d) Hyperphoria greater than 1 prism diopter.

(e) Near point of convergence (NPC) greater than $100 \mathrm{~mm}$.

(5) Color vision. (See ATB, Color Vision Testing and APL, Color Vision Abnormalities.)

(a) Five or more errors in reading the 14 test plates of the Pseudoisochromatic Plate (PIP) Set; or

(b) Any error in reading the nine test light pairs of the Farnsworth Lantern (FALANT) or the OPTEC 900 Color Vision Tester.

(6) Binocular depth perception (stereo acuity) worse than 40 seconds of arc. (See ATB, Depth Perception Testing and APL, Defective Depth Perception.)

(a) Any error in Group B of the AFVT (40 seconds of arc); or

(b) Any error in levels 1 through 7 of the 10 levels of three circles each in the Random Dot (RANDOT) Circles Test; or

(c) Any error in levels 1 through 9 of the 9 levels of four circles each in the Titmus Graded Circles Stereoacuity Test.

(7) Field of vision. Any scotoma, other than physiologic blindspot. (See ATB, Field of Vision Testing.)

(8) Night vision. As noted by history. (There is currently no definitive test or score.) Any ocular abnormalities resulting in decreased night vision must be referred to ophthalmology for confirmation. (See ATB, Night Vision.)

b. Classes 2/2F/3/4. Same as Class 1, except as listed below:

(1) Distant and near visual acuity. Uncorrected acuity worse than 20/400 in either eye at distance or near, or vision not correctable to $20 / 20$ in each eye as outlined in paragraph 4-12a(1) and (2). 
(2) Manifest refractive error. Refractive error of such magnitude that the individual cannot be fit with aviation spectacles.

(3) NPC of greater than $100 \mathrm{~mm}$. This is not disqualifying but must be referred to Ophthalmology or Optometry for evaluation. (See ATB, Ocular Motility Testing; APL, Excessive Phorias; and APL, Convergence Insufficiency.)

\section{4-13. Genitourinary}

The causes of medical unfitness for flying duty Classes $1 / 2 / 2 \mathrm{~F} / 3 / 4$ are the causes in paragraphs $2-14$ and $2-15$, plus the following:

$a$. History of persistent hematuria with greater than five red blood cells per high power field on routine analysis.

$b$. History of any metabolic abnormality of the urine, to include proteinuria, glycosuria, and hypercalcinuria.

c. Uncomplicated pregnancy is not disqualifying, but results in flying duty restrictions. (See APL, Pregnancy.) In uncomplicated pregnancies, flying is restricted to synthetic flight simulator training during the entire pregnancy; or multi-crew, multi-engine, non-ejection seat fixed wing aircraft during the 13th through 24th week of gestation. The requirement for physiological training is waived during pregnancy.

d. Complications of pregnancy. (See APL, Pregnancy.)

$e$. History of urinary tract stone formation or retention of urinary tract stone within collecting system. (See APL, Kidney Stones, and APL, Pregnancy.)

\section{4-14. Head and neck}

The causes of medical unfitness for flying duty Classes 1/2/2F/3/4 are the causes in paragraphs 2-16, 2-17, and 4-22.

\section{4-15. Heart and vascular system}

The causes of medical unfitness for flying duty Classes $1 / 2 / 2 \mathrm{~F} / 3 / 4$ are the causes in paragraphs $2-18$ and $2-19$, plus the following:

a. History of any abnormal electrocardiographic findings, including but not limited to:

(1) Left axis deviation greater than minus 45 degrees.

(2) Acquired right axis deviation greater than 120 degrees.

(3) First degree AV-block when the PR interval (interval between the $\mathrm{P}$ and $\mathrm{R}$ waves on an electrocardiogram (EKG)) cannot be shortened to less than or equal to 220 milliseconds in the unipolar leads during exercise.

(4) Mobitz Type II second degree AV block, and third degree AV block.

(5) Acquired left anterior or posterior hemiblock.

(6) Acquired complete right bundle branch block. (See APL, Acquired Right Bundle Branch Block.)

(7) Complete left bundle branch block.

(8) Pre-excitation as manifested by Wolff-Parkinson-White pattern or short PR interval (PR interval less than 120 milliseconds in all 12 leads). Wolff-Parkinson-White syndrome.

(9) Sinus pause or asystole accompanied by symptoms and/or greater than 2.2 seconds in duration.

(10) Bradydysrhythmias accompanied by symptoms and/or hypotension.

(11) Supraventricular tachycardia (3 or more beats at a rate greater than 100) to include atrial fibrillation/flutter, multifocal atrial tachycardia, junctional tachycardia, and persistent sinus tachycardia.

(12) Frequent uniform or multiform ventricular premature beats, or ventricular premature beats, or ventricular premature beat pairs, as defined by APL, Abnormal Electrocardiogram.

(13) Ventricular tachycardia (3 or more beats at a rate greater than 100), to include ventricular fibrillation/flutter and accelerated idioventricular rhythm.

(14) Acquired ST and T wave abnormalities consistent with myocardial dysfunction of any etiology.

(15) Aeromedically abnormal exercise treadmill test as defined by ATB, Aeromedical Graded Exercise Test, until reviewed by the USAAMA. (See APL, Abnormal Cardiac Function Testing.)

$b$. History of hypertrophic, dilated, or obstructive cardiomyopathy, to include left ventricular hypertrophy, as documented by clinical or EKG evidence. Hypertrophy due to athletic heart is not disqualifying. (See APL, Aeromedical Cardiovascular Screening Program.)

c. History of valvular heart disease, to include mitral valve prolapse, as documented by clinical or electrocardiographic findings.

$d$. History of myocarditis, or endocarditis, to include subacute bacterial endocarditis. History of pericarditis until reviewed by the USAAMA.

$e$. Any evidence of coronary artery disease as outlined by APL, Aeromedical Cardiovascular Screening Program.

$f$. For Classes $2 / 2 \mathrm{~F}$, suspected coronary artery disease such as an elevated cardiac risk index, elevated total cholesterol or cholesterol/high-density lipoprotein (HDL) -cholesterol ratio in conjunction with an abnormal aeromedical graded exercise treadmill test and/or abnormal cardiac fluoroscopy as outlined in APL, Aeromedical Cardiovascular Screening Program. (See also ATB 6, Aeromedical Graded Exercise Test, and ATB 9, Cardiac Fluoroscopy.)

$g$. History of congenital anomalies of the heart or great vessels, or surgery to correct these anomalies. 
$h$. History of cor pulmonale or congestive heart failure.

$i$. History of hypertension with a systolic pressure of $140 \mathrm{mmHg}$ or greater, and/or diastolic pressure of $90 \mathrm{mmHg}$ or greater, with or without systemic complications confirmed by average reading of a 3-day blood pressure check. (See APL, Hypertension in Aircrew Members.)

$j$. Orthostatic hypotension or orthostatic intolerance or symptomatic hypotension. (See para 4-22e.)

$k$. History of diseases of the blood and lymphatic vessels, to include but not limited to, aortic aneurysm, arteriosclerotic occlusive disorders, fistulas, vasculitis, vasospastic disorders, thromboembolic disorders, and lymphedema.

$l$. History of any cardiac surgical procedure, to include pacemaker insertion, valve replacement, bypass tract ablation by any method, coronary angioplasty, and coronary artery bypass.

\section{4-16. Linear anthropometric dimensions}

The causes of medical unfitness for flying duty Classes $1 / 2 / 2 \mathrm{~F} / 3 / 4$ are the following:

a. Initial Classes $1 / 2 / 2 F$. Failure to meet linear anthropometric standards. Total arm reach equal to or greater than $164.0 \mathrm{~cm}$. Sitting height equal to or less than $102.0 \mathrm{~cm}$. Crotch height equal to or greater than $75.0 \mathrm{~cm}$. (See ATB, Anthropometry.)

b. Class 3. Linear anthropometric measurements and body composition not compatible with aviation or crew member safety, or operational effectiveness at the Class 3 aircrew member's workstation.

\section{4-17. Weight and body build}

Aircrew members are medically unfit for flying duty Classes $1 / 2 / 2 \mathrm{~F} / 3 / 4$ when the body weight or build prevents normal functions required for safe and effective aircraft flight such as interference with aircraft instruments, controls, and aviation life support equipment, to include proper function of crash worthy seats, ejection seats, and other mechanisms of egress. (Military aircrew members may be subject to administrative restriction from flying duty by their commander when body weight or composition exceeds the limits prescribed by AR 600-9.)

\section{4-18. Lung and chest wall}

The causes of medical unfitness for flying duty Classes $1 / 2 / 2 \mathrm{~F} / 3 / 4$ are the causes in paragraphs $2-23$ and $4-2$, plus the following:

a. Pneumothorax, spontaneous.

(1) Class 1. A history of spontaneous pneumothorax.

(2) Classes $2 / 2 \mathrm{~F} / 3$.

(a) Single instance of spontaneous pneumothorax within the last 2 months, and until clinical evaluation shows complete recovery with full expansion of the lung, normal pulmonary function, and with no additional lung pathology, or other contraindication to flying.

(b) Recurrent spontaneous pneumothorax; waiver may be considered if effectively treated by pleuridesis and/or pleurectomy with complete recovery and successful completion of an altitude chamber ride to 18,000 feet.

$b$. Pneumothorax, traumatic, as outlined in $a(2)(a)$ above.

c. Pulmonary tuberculosis or tuberculous pleurisy; except chemoprophylaxis for tuberculin test conversion only is not disqualifying.

$d$. Presence of bullae.

e. Sarcoidosis. (See APL, Sarcoidosis.)

\section{4-19. Mouth}

The causes of medical unfitness for flying duty Classes $1 / 2 / 2 \mathrm{~F} / 3 / 4$ are the causes in paragraph $2-24$, plus the following:

a. Any infectious lesion until recovery is complete and the part is functionally normal.

$b$. Any congenital or acquired lesion that interferes with the function of the mouth or throat.

c. Any defect in speech that would prevent or interfere with clear and effective communication in the English language over a radio communication system.

$d$. Recurrent calculi of any salivary gland or duct.

\section{4-20. Nose}

The causes of medical unfitness for flying duty Classes $1 / 2 / 2 F / 3 / 4$ are in paragraph $2-25$, plus the following:

$a$. History of allergic rhinitis or vasomotor rhinitis requiring the use of antihistamines for a cumulative period greater than 30 days per year. (See APL, Allergic/Non-allergic Rhinitis.)

$b$. Deviation of the nasal septum or septal spurs that results in symptomatic obstruction of airflow, chronic rhinitis, chronic sinusitis, or interference of sinus drainage.

c. History of nasal polyps, or sinus polyps, or retention cysts. 
$d$. Acute, recurrent sinusitis or chronic sinusitis and/or surgery to treat chronic sinusitis.

\section{4-21. Pharynx, larynx, trachea, and esophagus}

The causes of medical unfitness for flying duty Classes $1 / 2 / 2 \mathrm{~F} / 3 / 4$ are in paragraph $2-25$, plus the following:

a. History of recurrent hoarseness interfering with communication.

$b$. History of tracheostomy.

c. History of chronic or recurrent eustachian tube dysfunction.

\section{4-22. Neurological disorders}

The causes of medical unfitness for flying duty Classes $1 / 2 / 2 \mathrm{~F} / 3 / 4$ are in paragraphs $2-26,2-29 d$, and $4-14$, plus the following (see table 4-2):

a. History of electroencephalographic abnormalities of any kind; to include spike-wave complexes, spikes, or sharp waves.

$b$. History of chronic, recurrent, or incapacitating headaches. (See APL, Headache and Migraine.)

c. History of neuritis, neuralgia, neuropathy, or radiculopathy until reviewed by the USAAMA.

$d$. History of decompression sickness (Type II) or an air embolism with neurologic involvement.

$e$. History of disturbances in consciousness, single episode or recurrent; to include nontraumatic loss of consciousness, narcolepsy, cataplexy, all forms of paroxysmal convulsive disorders, or single convulsive seizures of any type, except-

(1) Single episode of documented vasovagal syncope such as syncope with venipuncture or immunizations.

(2) Single episode of documented postural or parade-rest syncope, not otherwise disqualifying.

(3) Febrile seizures before the age of 5 with a normal EEG.

$f$. Central nervous system infections.

(1) Class 1. Within 1 year prior to examination, except 6 years for encephalitis, or if there are residual neurological deficits or other sequelae.

(2) Classes $2 / 2 \mathrm{~F} / 3$. Until complete recovery without residual neurological deficits or other sequelae.

$g$. History of organic mental syndromes; developmental, learning, or sensory processing disorders; or toxic or metabolic central nervous system disorders, until reviewed by the USAAMA.

$h$. History of intracranial embolism, vascular insufficiency, thrombosis, hemorrhage, arteriovenous malformation, or aneurysm.

$i$. History of degenerative or demyelinating process, such as multiple sclerosis, dementia, Alzheimer's disease, Parkinson's disease, or basal ganglia disease.

$j$. For Class 1, history of diseases with neurologic sequelae, such as hepatolenticular degeneration, neurofibromatosis, acute intermittent porphyria, or familial periodic paralysis.

$k$. History of benign or malignant neoplasms of the brain, pituitary gland, spinal cord, or their coverings.

l. History of diagnostic or therapeutic craniotomy, or any procedure involving penetration of the dura mater or the brain substance, including ventriculo-peritoneal shunts, evacuation of hematomas, and brain biopsy.

$m$. Any defect in the bony substance of the skull, regardless of cause.

$n$. History of any head injury associated with the following will be cause for permanent disqualification for aviation duty for all Classes. (See also table 4-2.)

(1) Intracranial hemorrhage or hematoma, to include epidural, subdural, intracerebral, or subarachnoid hemorrhage.

(2) Any penetration of the dura mater or brain substance.

(3) Radiographic or other evidence of retained intracranial foreign bodies or bony fragments.

(4) Transient or persistent neurological deficits indicative of parenchymal central nervous system injury, such as hemiparesis or cranial neuropathy.

(5) Persistent focal or diffuse abnormalities of the EEG reasonably assumed to be a result of an accident.

(6) Depressed skull fracture with or without dural penetration.

(7) Linear or basilar skull fracture with or without dural penetration.

(8) Posttraumatic syndrome as manifested by changes in personality, impairment of higher intellectual functions, anxiety, headaches, or disturbances of equilibrium that does not resolve within 6 weeks after injury.

(9) Unconsciousness exceeding 24 hours.

(10) Cerebrospinal fluid rhinorrhea or otorrhea persisting more than 7 days.

$o$. History of head injury associated with any of the following will be cause for permanent disqualification for flying duties for Class 1; and termination of aviation service for a minimum of 2 years for Classes $2 / 2 F / 3$. (See table $4-2$.)

(1) Linear or basilar skull fracture with loss of consciousness for more than 15 minutes but less than 2 hours.

(2) Posttraumatic syndrome, as manifested by changes in personality, impairment of higher intellectual functions, anxiety, headaches, or disturbances of equilibrium, that persists for more than 2 weeks, but resolves within 6 weeks of the injury. 
(3) Amnesia (posttraumatic and retrograde, patchy or complete), delirium, disorientation, or impairment of judgment that exceeds 24 hours.

(4) Unconsciousness for a period of greater than 2 hours, but less than 24 hours.

p. History of head injury associated with any of the following will be cause for a 2-year disqualification for Class 1 ; and temporary medical suspension from aviation duty for 3 months for Classes 2/2F/3. (See table 4-2.)

(1) Linear or basilar skull fracture with loss of consciousness for less than 15 minutes.

(2) Post-traumatic syndrome, as manifested by changes in personality, impairment of higher intellectual functions, anxiety, headaches, or disturbances of equilibrium, that persists for more than 48 hours but resolves within 14 days of the injury.

(3) Post-traumatic headaches alone that persist more than 14 days after injury, but resolve within 1 month.

(4) Amnesia (post-traumatic and retrograde, patchy and complete), delirium, or disorientation that lasts less than 24 hours, but more than 12 hours after injury.

(5) Unconsciousness for more than 15 minutes but less than 2 hours.

(6) Cerebrospinal fluid rhinorrhea or otorrhea that clears within 7 days of injury, provided there is no evidence of cranial nerve palsy.

q. History of head injury associated with any of the following will be cause for a 3-month disqualification for Class 1 , and temporary medical suspension from aviation duty for 1 month for Classes 2/2F/3.

(1) Post-traumatic syndrome, as manifested by changes in personality, impairment of higher intellectual functions, anxiety, headaches, or disturbances of equilibrium, that resolves within 48 hours of the injury.

(2) Post-traumatic headaches alone that resolves within 14 days after injury.

(3) Amnesia (post-traumatic and retrograde, patchy and complete), delirium, or disorientation that lasts less than 12 hours after injury.

(4) Unconsciousness less than 15 minutes.

\section{4-23. Mental disorders}

The minimum psychiatric evaluation will include Axis I, II, and III, using diagnostic criteria and terms found in DSM-IV. The causes of medical unfitness for flying duty Classes 1/2/2F/3/4 are the causes in paragraph 2-27 , except as modified by the following:

a. History of any psychotic episode evidenced by impairment in reality testing, to include transient disorders, from any cause except transient delirium secondary to toxic or infectious processes before age 12 .

$b$. History of mood disorder, to include major mood disorders, depression, cyclothymic, dysthymic, and mood disorders not otherwise specified.

c. History of anxiety disorder, somatoform disorder, or dissociative disorder, including but not limited to those disorders previously described as neurotic. History of any phobias or severe or prolonged anxiety episodes, after age 12, even if they do not meet the diagnostic criteria of DSM-IV.

$d$. History of factitious disorders and disorders of impulse control not elsewhere classified.

$e$. History of pervasive or specific developmental disorders usually first seen in childhood. Stuttering, sleepwalking, and sleep terror disorders if occurring after the 14th birthday.

$f$. History of personality or behavior disorder. Personality traits insufficient to meet DSM-IV criteria for personality disorder diagnosis may be cause for an unsatisfactory Aeromedical Adaptability (AA) rating (formerly Adaptability Rating for Military Aeronautics (ARMA)). (See para 4-29.)

g. History of any adjustment disorder until reviewed by the USAAMA.

$h$. Excessive alcohol use.

(1) History of alcohol abuse or dependence by DSM-IV criteria is disqualifying for all Classes.

(2) History of alcohol misuse may be disqualifying for all Classes. (See APL, Alcohol-Related Disorders, for aeromedical evaluation, treatment, and disposition guidelines. See also AR 600-85.)

$i$. Drug misuse, abuse, or dependence. History of misuse or abuse of any controlled substance, and/or use of any illicit drugs, including marijuana and psychoactive substances for all Classes. (See APL, History of Illicit Drug Use and para 2-31 also applies.)

$j$. History of suicide attempt or gesture at any time.

$k$. Insomnia, severe or prolonged.

l. Unconscious (neurotic) fear of flying manifested as psychiatric or somatic symptoms. Refer aircrew with a conscious fear of flying, that is, those who have made a conscious choice not to fly, to the aviation unit commander for a nonmedical disqualification and flying evaluation board (FEB). (See AR 600-105.)

$m$. Emotional responses to situations of stress, either combat or noncombat, when such a reaction may interfere with the efficient and safe performance of an individual's flying duties as determined by review by the USAAMA.

Note. (See APL, Mental Health Findings.) 


\section{4-24. Skin and cellular tissues}

The causes of medical unfitness for flying duty Classes $1 / 2 / 2 \mathrm{~F} / 3 / 4$ are the causes listed in paragraph $2-28$, plus any skin condition that interferes with the use of aviation clothing or life support equipment.

\section{4-25. Spine, scapula, ribs, and sacroiliac joints}

The causes of medical unfitness for flying duty Classes $1 / 2 / 2 \mathrm{~F} / 3 / 4$ are the causes listed in paragraphs $2-11$ and 2-29, plus the following:

a. History of chronic or recurrent disabling episodes of back pain, especially when associated with significant objective findings.

$b$. History of any fracture or dislocation of the vertebrae, to include insertion of spinal orthopedic hardware. A compression fracture involving less than 25 percent of a single vertebra is not disqualifying if the injury occurred more than 12 months ago and is asymptomatic; except any degree of compression fracture of the cervical vertebrae, twelfth thoracic vertebrae, or first lumbar vertebra. A history of fracture of the transverse or spinous process is not disqualifying if asymptomatic.

c. Scoliosis.

(1) Class 1. Any degree of scoliosis. Scoliosis may be qualified if the angulation is found to be stable by two standing scoliosis x-ray series done 12 months apart, and the scoliosis angle in the thoracic or lumbar spine is 20 degrees or less by the Cobb method.

(2) Classes $2 / 2 F / 3$. Standing scoliosis $\mathrm{x}$-ray series demonstrating an angle in the thoracic or lumbar spine that exceeds 20 degrees by the Cobb method.

\section{4-26. Systemic diseases}

The causes for medical unfitness for flying duty Classes $1 / 2 / 2 \mathrm{~F} / 3 / 4$ are the causes in paragraph $2-30$ and 2-32, plus the following: Diseases and conditions that, based upon sound aeromedical principles, may in any way affect or compromise the individual's health or well-being, flying safety, or mission completion. The local flight surgeon will make the initial determination and recommendations to the individuals' commander. The USAAMA will make the final determination of medical unfitness for flying duty.

\section{4-27. Malignant diseases and tumors}

The causes of medical unfitness for flying duty Classes $1 / 2 / 2 \mathrm{~F} / 3 / 4$ are the causes listed below:

a. Benign tumors, same as the causes listed in paragraphs $2-31 a$ and $4-22 k$.

$b$. History of any malignant tumor, except for basal cell carcinoma of the skin that has been removed. (See also APL, Cancer in Aircrew.)

\section{4-28. Sexually transmitted diseases}

The causes of medical unfitness for flying duty Classes $1 / 2 / 2 \mathrm{~F} / 3 / 4$ are the causes listed in paragraph 2-14ab.

\section{4-29. Aeromedical adaptability}

a. The cause of medical unfitness for flying duty for all Classes excluding civilian ATCs is an unsatisfactory AA (formerly ARMA) due to socio-behavioral factors that are considered unsuitable for or unadaptable to Army aeronautics. The unsatisfactory AA may be a manifestation of underlying psychiatric disease (see para 4-23) or may be accompanied by non-medical disqualifications. (See AR 600-105.) The unsatisfactory AA is not a diagnosis, but is a determination by the FS and aviation commander or supervisor of suitability or adaptability. An unsatisfactory AA may be revealed by interview, records review, command referral, security investigations, or other documented sources.

$b$. Until reviewed by the USAAMA, an unsatisfactory AA may exist if any of the conditions listed below are present. Trained aircrew with an unsatisfactory AA should also be referred to the aviation unit commander for administrative evaluation of nonmedical disqualifications and determination of fitness to retain the aircrew member's aeronautical rating or status. (See AR 600-105.) Psychological and psychiatric consultation will be obtained as required by the FS or the USAAMA. The aviation commander and FS will forward their evaluations and recommendations to the USAAMA to make a final recommendation of medical fitness for flying duties. The USAAMA will coordinate with the Chief, Army Aviation Branch, and aeromedical waiver authorities as required. When there is a question of observer bias or loss of objectivity, the USAAMA may obtain additional medical evaluations from other impartial FSs or medical consultants.

(1) Deliberate or willful concealment of significant and/or disqualifying medical conditions on medical history forms or during FS interview.

(2) An attitude toward flying that is clearly less than optimal; for example, the person appears to be motivated overwhelmingly by the prestige, pay, or other secondary gains rather than the skill, achievement, and professionalism of flying itself.

(3) Clearly noticeable personality traits such as immaturity, self-isolation, difficulty with authority, poor interpersonal relationships, impaired impulse control, or other traits that may interfere with group functioning as a team 
member in an operational aviation setting, even though there are insufficient criteria for a personality disorder diagnosis.

(4) Review of the history or medical records reveals multiple or recurring physical complaints that strongly suggest either a somatization disorder or a propensity for physical symptoms during times of stress. (See also para 4-23m.)

(5) A history of arrests, illicit drug use, or social "acting out" that may indicate immaturity, impulsiveness, or antisocial traits. Experimental use of drugs during adolescence, minor traffic violations, or clearly provoked impulsive episodes may be found fit after review by the USAAMA. (See also para 4-23i.)

(6) Significant prolonged or currently unresolved interpersonal or family problems, marital dysfunction, or significant family opposition or conflict concerning the Soldier's aviation career.

$c$. Until reviewed by the USAAMA, an unsatisfactory AA may be given for lower levels (symptoms and signs) than those mentioned in $b$ above if, in the opinion of the FS and aviation commander or civilian supervisor, mental or physical factors might be exacerbated under the stresses of Army aviation or the person might not be able to carry out his or her duties in a mature and responsible fashion. A person may be disqualified for any of a combination of factors listed in $b$ above and/or due to personal habits or appearance indicative of attitudes of carelessness, poor motivation, or other characteristics that may be unsafe or undesirable in the aviation environment.

\section{4-30. Reading Aloud Test}

The cause of medical unfitness for flying duty Classes 1/2/2F/3/4 is failure to clearly communicate in the English language in a manner compatible with safe and effective aviation operations. For initial applicants, this is determined by administration of the Reading Aloud Test. (See ATB 2, Army Flight Surgeon's Administrative Guide.) In questionable cases, the aviation unit commander, ATC supervisor, or other appropriate aviation official will provide a written recommendation to the FS.

\section{4-31. Department of the Army civilian and contract civilian aircrew members}

a. The following references apply as noted.

(1) 5 CFR Part 339, Office of Personnel Management, applies to DA civilians.

(2) AR 95-20/AFJI 10-220/NAVAIRINST 3710.1E/DCMA INST 8210.1 applies to contract civilian aircrew members who fly in aircraft owned or leased by DOD.

(3) 14 CFR Part 61 and 14 CFR Part 67, Federal Aviation Administration, do not apply since Army civilian aircrew members fly public use aircraft. The agency that owns or operates public use aircraft is responsible for the medical certification of aircrew flying those aircraft.

$b$. The aeromedical certification of civilian aircrew members has three major components:

(1) Examination method. The Army determines the scope of examination and the examiners as outlined in chapter 6, APLs, and ATBs.

(2) Aeromedical standard. The classes of medical standards for flying are listed in paragraph 4-2. The medical conditions that pertain to each specific medical standard for flying are contained in paragraphs 4-4 through 4-33.

(3) Aeromedical disposition. The Army makes the final determination of fitness for flying duties using the administrative procedures in chapter 6, APLs, and ATBs. The Army may require additional consultations, examinations, and tests before a final determination is made. Civilian aircrew members may submit other medical documents from health care providers of their choice. The USAAMA may consult DA-designated aeromedical consultants and the Army Aeromedical Consultant Advisory Panel (ACAP) as required. The USAAMA makes the final recommendation of aeromedical fitness to the civilian aircrew member waiver authority designated in paragraphs 6-21e and 6-21f. The recommendation considers the civilian aircrew member's medical condition, aircraft flown, mission and duties and deployability status. The recommendation may be qualified, disqualified with waiver, or medical termination from aviation service. The waiver authority grants or denies the aeromedical recommendation.

(a) DAC aircrew members granted medical termination from aviation service are referred by the supervisor aviation unit commander to the Civilian Personnel Office for assistance in reassignment to duties not to include flying (DNIF). The Office of Personnel Management makes the final determination of eligibility for medical disability.

(b) Contract civilian aircrew members granted medical termination from aviation service are referred by the Contracting Officer Representative to the contractor management for reassignment to DNIF or termination of employment.

$c$. The following exception applies to civilian aircrew members. Civilian aircrew members are not required to meet the requirements of the Army Weight Control Program (AR 600-9). However, maximal allowable weight and anthropometric measurements are necessary and shall be followed to permit normal function required for safe and effective aircraft flight without interfering with aircraft instruments or controls, aircraft egress, or proper function of crash worthy or ejection seat systems. (See para 4-33 for ATC personnel.)

\section{4-32. Medical standards for Class 3 personnel}

a. Initial and subsequent medical certification of Class 3 aircrew is conducted according to this regulation, and APLs and ATBs issued by the USAAMA. 
$b$. The attending FS/APA/AMNP/AME makes the initial determination of fitness for Class 3 flying duties.

c. The FS/APA/AMNP/AME will utilize the following guidelines for Class 3 waiver/suspension recommendations:

(1) Class 3 aircrew with a major physical or psychological disqualification will be recommended for suspension from flying duties. Other disqualifications may be waived for flying duties. The FS/APA/AMNP/AME will take into consideration the operational duties and responsibilities of Class 3 aircrew before recommending a waiver/suspension action to the aviation unit commander. Questionable cases will be referred to the USAAMA.

(2) A major physical or psychological defect in the operational aviation environment is defined as any defect that will-

(a) Interfere with duties requiring visual or auditory acuity, speech clarity, dexterity, or adequate range of motion.

(b) Interfere with wearing aviation life support equipment, or use of controls at their duty station.

(c) Reduce the ability to withstand rapid changes in atmospheric pressure or forces of acceleration.

(d) Increase the risk of sudden incapacitation, compromising personal health, aviation safety, mission completion, or deployability.

(e) Require medications or treatments that compromise flight safety or deployability.

(3) Alcohol/drug abuse or dependence requires AHRC or NGB waiver.

$d$. The local aviation unit commander or civilian waiver authority, as appropriate, will grant or deny the aeromedical recommendation for waiver or suspension.

\section{4-33. Medical standards for ATC personnel}

a. DAC and DA contract civilian ATCs.

(1) Medical qualification requirements for Department of the Army civilian Air Traffic Controllers are outlined in Office of Personnel Management Operating Manual: Qualification Standards for General Schedule Positions, GS-2152: Air Traffic Control Series, in accordance with Section 339.202, Title 5, Code of Federal Regulations.

(2) DA contract civilian ATCs may be required by their contractor employer to maintain a Class II Federal Aviation Administration (FAA) medical certification; but this certification is not required by DA or FAA for contract ATCs to control air traffic in DOD facilities (14 CFR 65.31, 33). The initial and subsequent determinations of medical fitness for ATC duties are made as outlined in this regulation. The contract will state that DA contract ATCs will meet the same medical qualification requirements as those for DA civilians set forth in (1) above.

b. Class 4 military ATCs. The causes for unfitness for Class 4 ATC duties are-

(1) Eye. (See paras 4-11 and 4-12.)

(2) Ear, nose, and throat. (See also para 4-7.)

(a) Unilateral or bilateral disease of the outer, middle, or inner ear that may interfere with the comfortable, efficient use of the standard headphone apparatus, with accurate perception of voice transmissions or spoken communications, or equilibrium.

(b) Disease or malformation of the mouth or throat that may interfere with enunciation and clear speech, to include stuttering or stammering. (See paras 4-6, 4-19, and 4-30.)

(c) Hearing loss that exceeds the standards in table $4-1$.

(d) Nose and sinuses. (See para 4-20.)

(3) Cardiovascular and blood pressure. (See para 4-15.)

(4) Neuropsychiatric. (See paras 4-22, 4-23, and 4-29.)

(5) Endocrine. (See para 4-9 and APL, Diabetes and Glucose Intolerance.)

(6) Musculoskeletal.

(a) Any deformity or condition of the spine or limbs, or absence of any extremity, digit, or any portion thereof, that may interfere with satisfactory and safe performance of duty.

(b) Any condition that predisposes to fatigue or discomfort induced by long periods of standing or sitting.

(7) Weight and body build. These factors must not interfere with the operation of ATC equipment, or the use of work place facilities such as office chair or staircase.

(8) HIV seropositivity. (Civilian employees: Normally, neither applicants for employment nor current employees may be required to be tested for the presence of the HIV antibody. Civilian employees are not disqualified based solely on the presence of the HIV virus. See AR 600-110 and ATB 2, Army Flight Surgeon's Administrative Guide.)

(9) Other medical conditions. Other organic, systemic, functional, or structural diseases, defects, or limitations that in the opinion of the attending FS may be a potential hazard to safety in the Air Traffic Control System, or predispose to sudden incapacitation or inability to adapt to stress. (See paras 4-26, 4-27, and 4-28.) A pertinent history and clinical evaluation including laboratory screening will be obtained, and when clinically indicated, special consultations and examinations will be accomplished and forwarded to the USAAMA for review.

(10) Medications. Unfitting for ATC duties and requires a waiver. (See APL, Medications.) 
Table 4-1

Acceptable audiometric hearing level for Army aviation and air traffic control

\begin{tabular}{l|l|l|l|l|l|l|l}
\hline Frequency $(\mathrm{HZ})$ & 500 & 1000 & 2000 & 3000 & 4000 \\
\hline Class 1 & 25 & 25 & 25 & 35 & 4500 & 45 \\
\hline Classes 2/2F/3/4 & 25 & 25 & 25 & 35 & 55 \\
\hline
\end{tabular}

Table 4-2

Head injury guidelines for Army aviation

Disposition by Class (Refer to the glossary for acronyms and abbreviations used)

\begin{tabular}{|c|c|c|c|c|}
\hline Class 1 & Perm DQ & Perm DQ & 2-year DQ & 3-month DQ \\
\hline Classes $2 / 2 F / 3 / 4$ & Perm DQ & 2-year DQ & 3-month DQ & 4-week DQ \\
\hline \multicolumn{5}{|l|}{ Problem: } \\
\hline - Intracranial bleeding & Any & - & - & - \\
\hline - Penetration of dura or brain & Any & - & - & - \\
\hline - Intracranial bone fragment or foreign bodies & Any & $\longrightarrow$ & $\longrightarrow$ & - \\
\hline $\begin{array}{l}\text { - Central nervous system deficits indicating } \\
\text { parenchymal injury }\end{array}$ & Any & - & - & - \\
\hline - EEG abnormality due to injury & Any & - & $\longrightarrow$ & - \\
\hline - Depressed skull fracture & Any & - & $\longrightarrow$ & - \\
\hline - Basilar or linear skull fracture with- & $\mathrm{LOC}>2 \mathrm{~h}$ & LOC $15 m-2 h$ & $\mathrm{LOC}<15 \mathrm{~m}$ & - \\
\hline - Post trauma syndrome lasting- & $>6 w k$ & $2 w k-6 w k$ & $48 h-14 d$ & $<48 h$ \\
\hline - Loss of consciousness lasting- & $>24 h$ & $2-24 h$ & $15 m-2 h$ & $<15 \mathrm{~m}$ \\
\hline - Cerebrospinal fluid leaking- & $>7 d$ & - & $<7 d$ & - \\
\hline - Amnesia, delirium, or disorientation lasting- & - & $>24 h$ & $12-24 h$ & $<12 \mathrm{~h}$ \\
\hline
\end{tabular}

Notes:

1. DQ: (aeromedical disqualification.

2 LOC: loss of consciousness.

\section{Chapter 5}

\section{Medical Fitness Standards for Miscellaneous Purposes}

\section{5-1. General}

This chapter sets forth medical conditions and physical defects that are causes for rejection for-

a. Airborne training and duty, Ranger training and duty, and Special Forces training and duty.

b. SERE training.

c. Freefall parachute training and duty.

d. Army service schools.

$e$. Diving training and duty.

$f$. Enlisted MOSs.

g. Geographical area assignments.

h. Deployment.

\section{5-2. Application}

The standards apply to applicants or individuals from all COMPOS under consideration for selection or retention in these programs, assignments, or duties.

5-3. Medical fitness standards for initial selection for Airborne training, Ranger training, and Special Forces training, and Reconnaissance and Surveillance Leaders Course training

The causes of medical unfitness for initial selection for Airborne training, Ranger training, Special Forces, and 
Reconnaissance and Surveillance Leaders Course (RSLC) training are all the causes listed in chapter 2, plus all the causes listed in paragraphs 5-3 and 5-4.

a. Abdomen and gastrointestinal system.

(1) Paragraph 2-3.

(2) Hernia of any variety including inguinal and other abdominal.

(3) Operation for relief of intestinal adhesions at any time.

(4) Laparotomy within a 6-month period.

(5) Chronic or recurrent gastrointestinal disorder.

(6) For Special Forces initial training, asplenia (absence of the spleen) for any reason.

b. Blood and blood-forming tissue diseases.

(1) Paragraph 2-4.

(2) Sickle cell disease.

c. Dental. Paragraph 2-5.

d. Ears and hearing.

(1) Paragraphs 2-6 and 2-7.

(2) Radical mastoidectomy.

(3) Any infectious process of the ear until completely healed.

(4) Marked retraction of the tympanic membrane if mobility is limited or if associated with occlusion of the Eustachian tube.

(5) Recurrent or persistent tinnitus.

(6) History of attacks of vertigo, with or without nausea, emesis, deafness, or tinnitus.

e. Endocrine and metabolic diseases. Paragraph 2-8.

f. Extremities.

(1) Paragraphs 2-9 through 2-11.

(2) Less than full strength and range of motion of all joints.

(3) Loss of any digit from either hand.

(4) Deformity or pain from an old fracture.

(5) Instability of any degree of major joints.

(6) Poor grasping power in either hand.

(7) Locking of a knee joint at any time.

(8) Pain in a weight-bearing joint.

(9) Retained hardware that is integral to maintaining fixation or stability, or presents a risk to mobility or a risk of further injury by its presence.

g. Eyes and vision.

(1) Paragraphs 2-12 and 2-13 with exceptions noted below.

(2) For Airborne and Ranger training: Distant visual acuity of any degree that does not correct to at least 20/20 in one eye and 20/100 in the other eye within 8 diopters of plus or minus refractive error, with spectacle lenses.

(3) For Special Forces training: Distant visual acuity of any degree that does not correct to 20/20 in both eyes with spectacle lenses. Any refractive error in spherical equivalent of worse than plus or minus 8 diopters.

(4) For Airborne and Special Forces training: Failure to pass the PIP set or FALANT test for color vision (see para 4-2a) unless the applicant is able to identify vivid red and/or vivid green as projected by the Ophthalmological Projector or the Stereoscope, Vision Testing (SVT).

h. Genitourinary system. Paragraphs 2-14 and 2-15.

i. Head and neck.

(1) Paragraphs 2-16 and 2-17.

(2) Loss of bony substance of the skull.

(3) Persistent neuralgia; tic douloureux; facial paralysis.

(4) A history of subarachnoid hemorrhage.

j. Heart and vascular system. Paragraphs 2-18 through 2-19, except for Special Forces training and duty: blood pressure with a preponderant systolic of less than $90 \mathrm{mmHg}$ or greater than $140 \mathrm{mmHg}$ or a preponderant diastolic of less than $60 \mathrm{mmHG}$ or greater than $90 \mathrm{mmHg}$, regardless of age. Unsatisfactory orthostatic tolerance test is also disqualifying.

k. Height. No special requirement.

l. Weight. No special requirement.

m. Body build. Paragraph 2-22.

$n$. Lungs and chest wall.

(1) Paragraph 2-23. 
(2) Spontaneous pneumothorax, except a single instance of spontaneous pneumothorax if clinical evaluation shows complete recovery with full expansion of the lung, normal pulmonary function, and no additional lung pathology or other contraindication to flying is discovered and the incident of spontaneous pneumothorax has not occurred within the preceding 3 months.

o. Mouth, nose, pharynx, larynx, trachea, and esophagus. Paragraphs 2-24 through 2-25.

p. Neurological disorders.

(1) Paragraph 2-26.

(2) Active disease of the nervous system of any type.

(3) Craniocerebral injury (see para 4-22m).

(4) Abnormal emotional responses to situations of stress (both combat and noncombat), when in the opinion of the medical examiner such reactions will interfere with the efficient and safe performance of the Soldier's duties.

q. Mental disorders.

(1) Paragraph 2-27.

(2) Individuals who are under treatment with any mood-ameliorating, tranquilizing, or ataraxic drugs for hypertension, angina pectoris, nervous tension, instability, insomnia, and so forth, and for a period of 4 weeks after the drug has been discontinued.

(3) Evidence of excessive anxiety, tenseness, or emotional instability. Fear of dark or enclosed spaces, fear of heights.

(4) Fear of flying when a manifestation of a psychiatric illness.

(5) History of psychosis or attempted suicide at any time.

(6) Phobias that materially influence behavior.

(7) Abnormal emotional response to situations of stress, when in the opinion of the medical examiner such reactions will interfere with the efficient and safe performance of duty.

r. Skin and cellular tissues. Paragraph 2-28.

s. Spine, scapulae, and sacroiliac joints.

(1) Paragraph 2-29.

(2) Scoliosis: lateral deviation of tips of vertebral spinous processes more than an inch.

(3) Spondylolysis; spondylolisthesis.

(4) Healed fractures or dislocations of the vertebrae.

(5) Lumbosacral or sacroiliac strain, or any history of a disabling episode of back pain, especially when associated with significant objective findings.

t. Systemic disease, general and miscellaneous conditions and defects.

(1) Paragraphs 2-30 and 2-32.

(2) Chronic motion sickness.

(3) Individuals who are under treatment with any of the mood ameliorating, tranquilizing, or ataraxic drugs and for a period of 4 weeks after the drug has been discontinued.

(4) Any severe illness, operation, injury, or defect of such a nature or of so recent occurrence as to constitute an undue hazard to the individual.

u. Tumors and malignant disease. Paragraph 2-31.

v. Sexually transmitted diseases. Paragraph 2-30.

\section{5-4. Medical fitness standards for selection for survival, evasion, resistance, escape training}

The causes of medical unfitness for SERE training are all the causes listed in chapter 3, plus all the causes listed in this paragraph.

a. Abdomen and gastrointestinal system. Paragraphs 2-3 and 3-5.

b. Blood and blood-forming tissue diseases. Paragraphs 3-7 and 3-42.

c. Dental. Paragraph 3-8.

d. Ears and hearing. Paragraphs 2-6, 2-7, 3-9, and 3-10.

e. Endocrine and metabolic diseases. Paragraphs 2-8b, 2-8c, 2-8h, 2-8j, and 3-11.

f. Extremities. Paragraphs 2-9b(8), 2-10b(3), 2-10b(6), 2-11c, 2-11d(2), 2-11e, and 3-12 through 3-14.

g. Eyes and vision. Paragraphs 3-15 and 3-16.

h. Genitourinary system. Paragraphs 2-14, 2-15, and 2-36.

i. Head and neck. Paragraph 5-3i.

j. Heart and vascular system. Paragraphs 2-18 and 2-19.

k. Height. No special requirements.

l. Weight. No special requirements.

m. Body build. Paragraph 2-22. 
n. Lungs and chest wall. Paragraph 2-23.

o. Mouth, nose, pharynx, larynx, trachea and esophagus. Paragraphs 2-24 and 2-25.

p. Neurological disorders.

(1) Paragraphs 2-26 and 4-22.

(2) Active disease of the nervous system of any type.

q. Mental disorders.

(1) Paragraph 2-27.

(2) Evidence of excessive anxiety, tenseness, or emotional responses to situations of stress (both combat and noncombat), when in the opinion of the medical examiner such reactions will interfere with the efficient and safe performance of the Soldier's duties.

r. Skin and cellular tissues. Paragraph 2-28.

s. Spine, scapulae, and sacroiliac joints. Paragraph 2-29.

t. Systemic disease and miscellaneous conditions and defects.

(1) Paragraphs $2-30$ and 2-32.

(2) Individuals who are under treatment with any of the mood ameliorating, tranquilizing, or ataraxic drugs and for a period of 4 weeks after the drug has been discontinued.

(3) Any severe illness, operation, injury, or defect of such a nature or of recent occurrence as to constitute an undue hazard to the individual.

u. Tumors and malignant diseases. Paragraph 2-31.

v. Sexually transmitted diseases. Paragraph 2-30.

5-5. Medical fitness standards for retention for Airborne duty, Ranger duty, and Special Forces duty Retention of an individual in Airborne duty, Ranger duty, and Special Forces duty will be based on-

$a$. His or her continued demonstrated ability to perform satisfactorily his or her duty as an Airborne officer or enlisted Soldier, Ranger, or Special Forces member.

$b$. The effect upon the individual's health and well-being by remaining on Airborne, Ranger, or Special Forces duty.

\section{5-6. Medical fitness standards for initial selection for free fall parachute training}

The causes of medical unfitness for initial selection for free fall parachute training are the causes listed in chapter 2 plus the causes listed in this paragraph and in paragraph 5-3.

a. Abdomen and gastrointestinal system. Paragraph 2-3.

$b$. Blood and blood-forming tissue diseases.

(1) Paragraph 2-4.

(2) Significant anemia or history of hemolytic disease due to variant hemoglobin state.

(3) Sickle cell disease.

(4) Sickle cell trait if the hemoglobin is less than $12.0 \mathrm{~g} . / \mathrm{dl}$., or there is any history of vasoocclusive crises.

c. Dental.

(1) Paragraph 2-5.

(2) Any unserviceable teeth until corrected.

d. Ears and hearing.

(1) Paragraphs 2-6 and 2-7.

(2) Abnormal labyrinthine function.

(3) Any infectious process of the ear, including external otitis, until completely healed.

(4) History of attacks of vertigo with or without nausea, emesis, deafness, or tinnitus.

(5) Marked retraction of the tympanic membrane if mobility is limited or if associated with occlusion of the Eustachian tube.

(6) Perforation, marked scarring, or thickening of the ear drum.

e. Endocrine and metabolic diseases. Paragraph 2-8.

f. Extremities.

(1) Paragraphs 2-9 through 2-11.

(2) Any limitation of motion of any joint that might compromise safety.

(3) Any loss of strength that might compromise safety.

(4) Instability of any degree or pain in a weight-bearing joint.

(5) Retained hardware that is integral to maintaining fixation or stability, or presents a risk to mobility or a risk of further injury by its presence.

g. Eyes and vision.

(1) Paragraphs 2-12 and 2-13, with exceptions noted in (2) and (3) below. 
(2) Uncorrected near visual acuity (14 inches) of worse than 20/50 in the better eye. Uncorrected distant visual acuity of worse than 20/100 in either eye. Distant vision that does not correct to 20/20 in both eyes with spectacle lenses. Any refractive error worse than plus or minus 8 diopters.

(3) Failure to pass the PIP or FALANT test for color vision unless the applicant is able to identify vivid red and vivid green as projected by the Ophthalmological Projector or the SVT.

(4) Any history of Laser-Assisted in situ Keratomileusis (LASIK) surgery is disqualifying.

h. Genitourinary system. Paragraphs $2-14$ and 2-15.

i. Head and neck.

(1) Paragraphs $2-16$ and 2-17.

(2) Loss of bony substance of the skull if retention of personal protective equipment is affected.

(3) A history of subarachnoid hemorrhage.

j. Heart and vascular system. Paragraphs $2-18$ and 2-19, except blood pressure with a preponderant systolic of less than $90 \mathrm{mmHg}$ or greater than $140 \mathrm{mmHg}$ or a preponderant diastolic of less than $60 \mathrm{mmHg}$ or greater than $90 \mathrm{mmHg}$ regardless of age. An unsatisfactory orthostatic tolerance test is also disqualifying.

k. Height. Paragraph 2-20.

l. Weight. Paragraph 2-21.

m. Body build. Paragraph 2-22.

$n$. Lungs and chest wall.

(1) Paragraph 2-23.

(2) Congenital or acquired defects that restrict pulmonary function, cause air-trapping, or affect ventilation-perfusion.

(3) Spontaneous pneumothorax, except a single occurrence at least 3 years before the date of the examination with clinical evaluation showing complete recovery with normal pulmonary function.

o. Mouth, nose, pharynx, larynx, trachea, and esophagus. Paragraphs 2-24 and 2-25.

p. Neurological disorders.

(1) Paragraphs 2-26.

(2) The criteria outlined in paragraph 4-22 for Classes 2 and 3 flying duty apply.

q. Mental disorders.

(1) Paragraph 2-27.

(2) Individuals who are under treatment with any of the mood ameliorating, tranquilizing, or ataraxic drugs for hypertension, angina pectoris, nervous tension, instability, insomnia, etc., and for a period of 4 weeks after the drug has been discontinued.

(3) Evidence of excessive anxiety, tenseness, or emotional instability.

(4) Fear of flying when a manifestation of a psychiatric illness.

(5) History of psychosis or attempted suicide at any time.

(6) Phobias that materially influence behavior.

(7) Abnormal emotional response to situations of stress, when in the opinion of the medical examiner such reactions will interfere with the efficient and safe performance of duty.

r. Skin and cellular tissues. Paragraph 2-28.

s. Spine, scapulae, ribs, and sacroiliac joints.

(1) Paragraph 2-29.

(2) Spondylolysis; spondylolisthesis.

(3) Healed fracture or dislocation of the vertebrae except mild, asymptomatic compression fracture.

(4) Lumbosacral or sacroiliac strain when associated with significant objective findings.

t. Systemic disease, general and miscellaneous conditions and defects.

(1) Paragraphs 2-30 and 2-32.

(2) History of motion sickness, other than isolated instances without emotional involvement.

(3) Any severe illness, operation, injury, or defect of such a nature or of so recent an occurrence as to constitute an undue hazard to the individual or compromise safe performance of duty.

u. Tumors and malignant diseases. Paragraph 2-31.

v. Sexually transmitted diseases. Paragraph 2-30.

\section{5-7. Medical fitness standards for retention for free fall parachute duty}

Retention of an individual in free fall parachute duty will be based on-

$a$. The Soldier's demonstrated ability to satisfactorily perform free fall parachute duty.

$b$. The effect upon the individual's health and well-being by remaining on free fall parachute duty. 
c. Determination of whether of any severe illness, operation, injury, or defect is of such a nature or of such recent occurrence as to constitute an undue hazard to the individual or compromise safe performance of duty.

\section{5-8. Medical fitness standards for Army service schools}

Except as provided elsewhere in this regulation, medical fitness standards for Army service schools are covered in other various Army Regulations.

\section{5-9. Medical fitness standards for initial selection for marine diving training (Special Forces and Ranger combat diving)}

The causes of medical unfitness for initial selection for marine self-contained underwater breathing apparatus (SCUBA) diving training are the causes listed in chapter 2, plus the following:

a. Abdomen and gastrointestinal system. Paragraph 2-3.

b. Blood and blood-forming tissue diseases.

(1) Paragraph 2-4.

(2) Significant anemia or history of hemolytic disease due to variant HGB state.

(3) Sickle cell disease.

c. Dental.

(1) Paragraph 2-5.

(2) Any infectious process and any conditions that contribute to recurrence until eradicated.

(3) Edentia; any unserviceable teeth until corrected.

(4) Moderate malocclusion extensive restoration or replacement by bridges or dentures that interfere with the use of SCUBA. Residual teeth and fixed appliances must be sufficient to allow the individual to easily retain a SCUBA mouthpiece.

d. Ears and hearing.

(1) Paragraphs 2-6 and 2-7.

(2) Persistent or recurrent abnormal labyrinthine function as determined by appropriate tests.

(3) Any infectious process of the ear, including external otitis, until completely healed.

(4) History of attacks of vertigo with or without nausea, emesis, deafness, or tinnitus.

(5) Marked retraction of the tympanic membrane if mobility is limited or if associated with occlusion of Eustachian tube. (See pressure test requirement in $w$, below.)

(6) Perforation, marked scarring, or thickening of the eardrum.

e. Endocrine and metabolic diseases. Paragraph 2-8.

f. Extremities.

(1) Paragraphs 2-9 through 2-11.

(2) Any limitation of motion of any joint that might compromise safety.

(3) Any loss of strength that might compromise safety.

(4) Instability of any degree or pain in a weight-bearing joint.

(5) History of osteonecrosis (aseptic necrosis of the bone) of any type.

(6) Retained hardware that is integral to maintaining fixation or stability, or presents a risk to mobility, or a risk of further injury by its presence.

g. Eyes and vision.

(1) Paragraphs 2-12 and 2-13, with exceptions noted in (2) and (3), below.

(2) Distant visual acuity that does not correct to $20 / 20$ in both eyes with spectacle lenses. Any refractive error in spherical equivalent of worse than plus or minus 8 diopters.

(3) Failure to pass the PIP set or FALANT test for color vision unless the applicant is able to identify vivid red and/ or vivid green as projected by the Ophthalmological Projector or the SVT.

(4) Any history of Laser-Assisted in Situ Keratomileusis (LASIK) surgery is disqualifying.

h. Genitourinary system. Paragraphs 2-14 and 2-15.

i. Head and neck.

(1) Paragraphs 2-16 and 2-17.

(2) Loss of bony substance of the skull if retention of personal protective equipment is affected.

(3) History of subarachnoid hemorrhage.

j. Heart and vascular system. Paragraphs 2-18 and 2-19, except blood pressure with a preponderant systolic of less than $90 \mathrm{mmHg}$ or greater than $140 \mathrm{mmHg}$ or a preponderant diastolic of less than $60 \mathrm{mmHg}$ or greater than $90 \mathrm{mmHg}$, regardless of age. An unsatisfactory orthostatic tolerance test is also disqualifying.

k. Height. Paragraph 2-20.

l. Weight. The individual must meet the weight standards prescribed by AR 600-9. The medical examiner may impose body fat measurements not otherwise requested by the commander. 
m. Body build.

(1) Paragraph 2-22.

(2) Obesity of any degree.

$n$. Lungs and chest wall.

(1) Paragraph 2-23.

(2) Congenital or acquired defects that restrict pulmonary function, cause air-trapping, or affect ventilation or perfusion.

(3) Spontaneous pneumothorax, except a single occurrence at least 3 years before the date of the examination with clinical evaluation showing complete recovery with normal pulmonary function.

o. Mouth, nose, pharynx, larynx, trachea, and esophagus. Paragraphs 2-24 and 2-25.

p. Neurological disorders.

(1) Paragraph 2-26.

(2) The criteria outlined in paragraph 4-22 for Classes 2 and 3 flying duty apply.

q. Psychotic disorders. Learning, psychiatric, and behavioral disorders. Disorders with psychotic features, affective disorders (mood disorders), anxiety, somatoform, or dissociative disorders (neurotic disorders).

(1) Paragraph 2-27.

(2) Individuals who are under treatment with any of the mood ameliorating, tranquilizing, or ataraxic drugs for hypertension, angina pectoris, nervous tension, instability, insomnia, etc, and for a period of 4 weeks after the drug has been discontinued.

(3) Evidence of excessive anxiety, tenseness, or emotional instability.

(4) Fear of flying when a manifestation of a psychiatric illness.

(5) History of psychosis or attempted suicide at any time.

(6) Phobias that materially influence behavior.

(7) Abnormal emotional response to situations of stress, when in the opinion of the medical examiner such reactions will interfere with the efficient and safe performance of duty.

(8) Fear of depths, enclosed places, or of the dark.

r. Skin and cellular tissues. Paragraph 2-28.

s. Spine, scapulae, ribs, and sacroiliac joints. (Consultation with an orthopedist and, if available, a diving medical officer (DMO) will be obtained in questionable cases.)

(1) Paragraph 2-29.

(2) Spondylolisthesis; spondylolysis that is symptomatic or likely to interfere with diving duty.

(3) Healed fracture or dislocation of the vertebrae except a mild, asymptomatic compression fracture.

(4) Lumbosacral or sacroiliac strain when associated with significant objective findings.

t. Systemic disease, general and miscellaneous conditions and defects.

(1) Paragraphs 2-30 and 2-32.

(2) Chronic motion sickness.

(3) Any severe illness, operation, injury, or defect of such a nature or of so recent an occurrence as to constitute an undue hazard to the individual or compromise safe performance of duty.

u. Tumors and malignant diseases. Paragraph 2-31.

v. Sexually transmitted diseases. Paragraph 2-30.

w. Pressure equalization and oxygen intolerance. If a hyperbaric chamber is available, examinees will be tested for the following disqualifying condition: Failure to equalize pressure. All candidates will be subjected, in a compression chamber, to a pressure of 27 pounds (12.15 kilogram (60 feet) per square inch to determine their ability to withstand the effects of pressure, to include ability to equalize pressure on both sides of the eardrums by Valsalva or similar maneuver. This test should not be performed in the presence of a respiratory infection that may temporarily impair the ability to equalize or ventilate.

\section{5-10. Medical fitness standards for retention for marine diving duty (Special Forces and Ranger combat diving)}

Retention of a Soldier in marine diving duty (SCUBA) will be based on-

a. The Soldier's demonstrated ability to satisfactorily perform marine (SCUBA) diving duty.

$b$. The effect upon the Soldier's health and well being by remaining on marine (SCUBA) diving duty.

c. Determination of whether of any severe illness, operation, injury, or defect is of such a nature or of such recent occurrence as to constitute an undue hazard to the individual or compromise safe performance of duty.

\section{5-11. Medical fitness standards for initial selection for other marine diving training (MOS 00B)}

The causes of medical unfitness for initial selection for diving training are all of the causes listed in chapter 2, plus the following: 
a. Abdomen and gastrointestinal system.

(1) Paragraph 2-3.

(2) Hernia of any variety.

(3) Operation for relief of intestinal adhesions at any time.

(4) Chronic or recurrent gastrointestinal disorder that may interfere with or be aggravated by diving duty. Severe colitis, peptic ulcer disease, pancreatitis, and chronic diarrhea do not meet the standard unless asymptomatic on an unrestricted diet for 24 months with no radiographic or endoscopic evidence of active disease or severe scarring or deformity.

(5) Laparotomy or celiotomy within the preceding 6 months.

b. Blood and blood-forming tissue diseases.

(1) Paragraph 2-4.

(2) Sickle cell disease.

(3) Significant anemia or history of hemolytic disease due to variant HGB state.

c. Dental.

(1) Paragraph 2-5.

(2) Any infectious process and any conditions that contribute to recurrence until eradicated.

(3) Edentia; any unserviceable teeth until corrected.

(4) Moderate malocclusion, extensive restoration, or replacement by bridges or dentures that interferes with the use of SCUBA. Residual teeth and fixed appliances must be sufficient to allow the individual to easily retain a SCUBA mouthpiece.

d. Ears and hearing.

(1) Paragraphs 2-6 and 2-7.

(2) Perforation, marked scarring, or thickening of the eardrum.

(3) Inability to equalize pressure on both sides of the eardrums by Valsalva or similar maneuver. See paragraph $5-9 w$.

(4) Acute or chronic disease of the auditory canal, tympanic membrane, middle or internal ear.

(5) Audiometric average level for each ear not more than $25 \mathrm{~dB}$ at 500, 1000, and $2000 \mathrm{~Hz}$ with no individual level greater than $30 \mathrm{~dB}$. Not over $45 \mathrm{~dB}$ at $4000 \mathrm{~Hz}$.

(6) History of otitis media or otitis externa with any residual effects that might interfere with or be aggravated by diving duty.

e. Endocrine and metabolic disease. Paragraph 2-8.

f. Extremities.

(1) Paragraphs 2-9 through 2-11.

(2) History of chronic or recurrent orthopedic pathology that would interfere with diving duty.

(3) Loss of any digit or portion thereof of either hand that significantly interferes with normal diving duty.

(4) Fracture or history of disease or operation involving any major joint until reviewed by a DMO.

(5) Any limitation of strength or range of motion of any of the extremities that would interfere with diving duties. g. Eyes and vision.

(1) Paragraph 2-12.

(2) Distant visual acuity, uncorrected, 20/200; not correctable to 20/20, each eye.

(3) Near visual acuity, uncorrected, of less than 20/50 or not correctable to 20/20.

(4) Failure to pass the PIP Set or FALANT test for color vision, unless the applicant is able to identify vivid red and vivid green as projected by the Ophthalmological Projector or the SVT.

(5) Abnormalities of any kind noted during ophthalmoscopic examination that significantly affect visual function or indicate serious systemic disease.

h. Genitourinary system.

(1) Paragraphs $2-14$ and 2-15.

(2) Chronic or recurrent genitourinary disease or complaints including glomerulonephritis and pyelonephritis.

(3) Abnormal findings by urinalysis, including significant proteinuria and hematuria.

(4) Varicocele, unless small and asymptomatic.

i. Head and neck. Paragraphs 2-16, 2-17, and 4-14.

j. Heart and vascular system.

(1) Paragraphs 2-18 and 2-19.

(2) Varicose veins that are symptomatic or may become symptomatic as a result of diving duty; deep vein thrombophlebitis; gross venous insufficiency.

(3) Marked or symptomatic hemorrhoids.

(4) Any circulatory defect (shunts, stasis, and others) resulting in increased risk of decompression sickness. 
(5) Persistent tachycardia or arrhythmia except for sinus type.

k. Height. Less than 66 or more than 76 inches.

l. Weight. Weight related to height that is outside the limits prescribed by AR 600-9.

m. Body build.

(1) Paragraph 2-22.

(2) Even though the Soldier's weight or body composition is within the limits prescribed by AR 600-9, he or she will be found medically unfit if the examiner considers that his or her weight or associated conditions in relationship to the bony structure, musculature, and/or total body fat content would adversely affect diving safety or endanger the Soldier's well-being if permitted to continue in diving status.

n. Lungs and chest wall.

(1) Paragraph 2-23.

(2) Congenital or acquired defects that restrict pulmonary function, cause air trapping, or affect ventilation-perfusion ratio.

(3) Any chronic obstructive or restrictive pulmonary disease at the time of examination.

o. Mouth, nose, pharynx, larynx, trachea, and esophagus.

(1) Paragraphs 2-24 and 2-25.

(2) History of chronic or recurrent sinusitis at any time.

(3) Any nasal or pharyngeal respiratory obstruction.

(4) Chronically diseased tonsils until removed.

(5) Speech impediments of any origin;, any condition that interferes with the ability to communicate clearly in the English language.

p. Neurological disorders.

(1) Paragraph 2-26.

(2) The special criteria that are outlined in paragraph 4-22 for Class 1 flying duty are applicable to diving duty.

q. Mental disorders.

(1) Paragraphs 2-27 through 2-31.

(2) The special criteria that are outlined in paragraph 4-23 for Class 1 flying duty are applicable to diving duty.

(3) The Military Diving Adaptability Rating may be considered MDAR satisfactory if the applicant meets the standards of paragraph 4-29 with the addition of having no fear of depths, enclosed places, or of the dark.

$r$. Skin and cellular tissues. Any active or chronic disease of the skin.

s. Spine, scapulae, ribs, and sacroiliac joints.

(1) Paragraph 2-33.

(2) Spondylolysis; spondylolisthesis.

(3) Healed fractures or dislocations of the vertebrae until reviewed by a DMO.

(4) Lumbosacral or sacroiliac strain, or any history of a disabling episode of back pain, especially when associated with significant objective findings.

t. Systemic diseases and miscellaneous conditions and defects.

(1) Paragraphs 2-34 and 2-35.

(2) Any severe illness, operation, injury, or defect of such a nature or of so recent occurrence as to constitute an undue hazard to the individual or compromise safe diving.

u. Tumors and malignant diseases. Paragraph 2-36.

v. Sexually transmitted diseases.

(1) Active sexually transmitted disease until adequately treated.

(2) History of clinical or serological evidence of active or latent syphilis, unless adequately treated, or of cardiovascular or central nervous system involvement at any time. Serological test for syphilis required.

w. Oxygen intolerance. See paragraph 5-9w.

\section{5-12. Medical fitness standards for retention for other marine diving duty (MOS 00B)}

The medical fitness standards contained in paragraph 5-11 apply to all personnel performing diving duty except that divers of long experience and a high degree of efficiency must-

$a$. Be free from disease of the auditory, cardiovascular, respiratory, genitourinary, and gastrointestinal systems.

$b$. Maintain their ability to equalize air pressure.

c. Have visual acuity, near and far, that corrects to $20 / 30$ in the better eye.

\section{5-13. Asplenic Soldiers}

a. Asplenic Soldiers are disqualified from initial training and duty in military specialties involving significant occupational exposure to dogs or cats. 
b. Asplenic Soldiers are disqualified from initial Special Forces training.

\section{5-14. Medical fitness standards for deployment and certain geographical areas}

a. All Soldiers considered medically qualified for continued military status and medically qualified to serve in all or certain areas of the continental United States (CONUS) are medically qualified to serve in similar or corresponding areas outside the continental United States (OCONUS).

$b$. Because of certain medical conditions, some Soldiers may require administrative consideration when assignment to combat areas or certain geographical areas is contemplated. Such consideration of their medical conditions would ensure these Soldiers are used within their functional capabilities without undue hazard to their health and well-being as well as ensure they do not produce a hazard to the health or well-being of other Soldiers.

c. Active duty Soldiers who do not meet the medical retention standards in chapter 3 of this regulation must be referred to an MEB/PEB for a fitness-for-duty determination. RC Soldiers not on active duty, who do not meet medical retention standards are referred for a fitness determination in accordance with paragraphs 9-10 (USAR) and 10-26 (NGB). Soldiers (RC or Active Army) with a permanent 3 or 4 in the physical profile who meet or might meet medical retention standards must be referred to an MMRB to determine if they are world-wide deployable (or be granted a waiver according to AR 600-60). However, Soldiers returned to duty by an MMRB or PEB, retained in the RC under paragraphs $9-10$ or $10-26$, or Soldiers with temporary medical conditions may still have some assignment/deployment limitations that must be considered before a decision is made to deploy.

$d$. The final decision to deploy a Soldier with certain medical conditions is a command decision, based on the health care provider's (HCP's) recommendations and taking into account the geographical and environmental conditions the Soldier will be subject to and the mission requirements the Soldier will be assigned. A Soldier with a temporary profile may deploy after the temporary disqualification expires or when the commander and the HCP agree the Soldier is deployable. When HCPs and unit commanders disagree on the deployability status of a Soldier, the decision will be raised to the first general officer in the Soldier's chain of command, who will review the case and make the final decision. For Soldiers with conditions listed in current DOD guidance or the combatant command's (COCOM's) published medical screening criteria, the general officer recommending deployment must submit a waiver request through the COCOM surgeon to the combatant commander for approval. If the Soldier has a temporary profile, it should be determined that the profile complies with the time limitations (including extensions) of AR 40-501. Soldiers with a permanent profile 3 or 4 may not deploy without a record of retention by a MMRB or a PEB unless the MMRB Convening Authority waives this requirement. In all cases, the role of the commander is to ensure Soldiers do not violate their profiles and are assigned duties that they can perform without undue risk to health and safety. Family member screening prior to overseas assignment will be completed according to AR 608-75, using DA Form 5888 (Family Member Deployment Screening Sheet).

$e$. It is the commander's responsibility to counsel Soldiers with physical profiles that may affect their deployment status. Medical guidance is critical in advising commanders of potential problems, physical limitations and potential situations that could be harmful to the Soldier or detrimental to the mission. Medical guidance is provided in the form of physical profiles documented on DA Form 3349 after being evaluated by an HCP. Soldiers will meet medical fitness standards in accordance with AR 40-501. Some Soldiers, because of certain medical conditions, may require administrative consideration when assigned to combat areas or certain geographic areas. As listed in AR 40-501, table 7-2, Profile codes, profiles address only physical functional capacity and limitations. Determination of a Soldier's assignment or duties, however, is the commander's responsibility and is outside of medical recommendations. As such, it is a commander's responsibility to counsel Soldiers on those duties they may or may not perform while deployed to combat areas or certain geographic areas. The counseled Soldiers will be advised that they will not violate their profiles and will perform duties assigned by the commander which they can perform without undue risk to health and safety.

$f$. See the DCS, G-1 personnel policy guidance message for additional deployment requirements on the DCS, G-1 Web site at http://www.armyg1.army.mil/militarypersonnel/ppg.asp. Medical standards for deployment are meant as general guides. The following medical conditions must be reviewed carefully by the medical provider before making a recommendation as to whether the Soldier can deploy to duty in a combat zone or austere isolated area where medical treatment may not be readily available.

(1) Diabetes requiring insulin. This requires an MEB/PEB (or for the RC, processing under paras 9-10 and 10-26). If found fit for duty, the Soldier should not deploy to areas where insulin cannot be properly stored (stored above freezing level but at less than 86 degrees Fahrenheit) or appropriate medical support cannot be reasonably assured. Deployment should only follow predeployment review and recommendation by an endocrinologist.

(2) Diabetes requiring oral medication for control. This requires a MEB/PEB (or for the RC processing under paras 9-10 and 10-26). If found fit for duty by a PEB, the Soldier may or may not be worldwide deployable (see table 5-1 for medical qualifications). 
Table 5-1

Guidance on deployment of Soldiers with diabetes

\begin{tabular}{|c|c|c|}
\hline Factor & OK to Deploy & Should Not Be Deployed \\
\hline Hgb A1C (for patient) & At target & Not at target \\
\hline Monofilament discrimination & Present & Absent \\
\hline Autonomic neuropathy & Absent & Present \\
\hline Knowledge of sick day rules & Sufficient & Insufficient \\
\hline Proliferative diabetic retinopathy & Absent & Present \\
\hline Macular edema & Absent & Present \\
\hline $\begin{array}{l}\text { Severe hypoglycemia (an episode requiring an- } \\
\text { other person's assistance) }\end{array}$ & Infrequent & Frequent \\
\hline $\begin{array}{l}\text { History of diabetic ketoacidosis in previous } 6 \\
\text { mos. }\end{array}$ & No & Yes \\
\hline Self-management skills & Good & Poor \\
\hline Hypoglycemia unawareness & Absent & Present \\
\hline $\begin{array}{l}\text { Parameters of permanent profile can be fol- } \\
\text { lowed }\end{array}$ & Yes & No \\
\hline $\begin{array}{l}\text { Significant co-morbidities (for example, conges- } \\
\text { tive heart failure, chronic kidney disease, signif- } \\
\text { icant coronary artery disease, poorly controlled } \\
\text { hypertension) requiring intensive management }\end{array}$ & Absent & Present \\
\hline $\begin{array}{l}\text { Risk of hypoglycemia is high if meals are } \\
\text { missed or delayed }\end{array}$ & No & Yes \\
\hline $\begin{array}{l}\text { Duty will place the Soldier in an OCONUS-Iso- } \\
\text { lated area where appropriate medical care and } \\
\text { means to monitor and support him/her are not } \\
\text { available }\end{array}$ & No & Yes \\
\hline
\end{tabular}

(3) Cardiovascular conditions. Review paragraphs 3-21 through 3-24 to determine if an MEB/PEB/trial of duty (or processing under paras 9-10 and 10-26 ) is necessary. If the Soldier successfully completes a trial of duty and is found fit for duty (or returned to duty by a Medical MOS Retention Board (MMRB)), the Soldier should not be deployed with any of the following conditions-

(a) Hypertension not controlled with medication.

(b) Recent episodes necessitating emergency room visits or closely monitored follow-up care.

(c) Permanent pacemakers, implanted antitachycardia and automatic implantable cardiac defibrillator (AICD) devices, and similar newly developed implanted cardiac devices require regular monitoring for battery life and functionality and should not be exposed to high electromagnetic fields. These particular devices have upper extremity physical limitations.

(d) Conditions requiring anticoagulants.

(e) Bare metal coronary stents. If the Soldier is found fit for duty by a PEB, the Soldier must remain at a location with access to a medical facility with the capability to do an urgent/emergent cardiac catheterization and laboratory monitoring of anti-platelet therapy for 3 months from the date of stent placement.

$(f)$ Drug eluting coronary stents. If the Soldier is found fit for duty by a PEB, the Soldier must remain at a location with access to a medical facility with the capability to do an urgent/emergent cardiac catheterization and laboratory monitoring of anti-platelet therapy for 6 months from the date of stent placement.

(g) Closure device for patent foramen ovale or atrial septal defect. If the Soldier is found fit for duty by a PEB, the Soldier must remain at a location with access to a medical facility with echocardiography, cardiology and laboratory monitoring of anti-platelet therapy capabilities for 6 months from the date of the closure of the defect.

(h) Cardiac arrhythmias. If the Soldier has undergone an electrophysiology ablation procedure and the Soldier is found fit for duty by a PEB, the Soldier must remain at a location with access to cardiac monitoring capabilities for 6 months from the date of the ablation procedure.

(i) Smallpox vaccine associated myocarditis. If after an evaluation by a cardiovascular specialist, the Soldier is found to meet medical retention standards, the Soldier must remain at a location with access to echocardiography and medical monitoring for 6 months from the date myocarditis was diagnosed.

(4) Neurological conditions. Review paragraph 3-30 for profile guidance and MEB/PEB processing criteria.

(a) Seizure disorders. If the Soldier is controlled with medications and seizure free for one year, the Soldier may be 
assigned overseas but should not be assigned to areas where access to medications or where the ability to monitor anticonvulsant drug levels are not available.

(b) Demyelinating conditions. If a Soldier has been found fit for duty by a PEB (see paragraph 3-30e) for stable non-disqualifying demyelinating disease, the Soldier should not be subjected to austere environments or where there is no readily available access to environments of equal to or less than 80 degrees Fahrenheit.

(5) Asthma. See paragraph 3-27a for profile guidance and for MEB/PEB processing criteria. If it is determined that the Soldier can be returned to duty, the Soldier should not deploy if he/she cannot wear protective gear, has experienced recent emergency room visits, or requires repetitive use of oral corticosteroids.

(6) Sleep apnea. See paragraph 3-41c for profile guidance and for MEB processing criteria. The Soldier can be deployed if nasal continuous positive airway pressure (CPAP) is required and can be supported in the area of deployment. Criteria for the ability to use nasal CPAP in the area of deployment include the following: availability of a reliable power source; absence of environmental factors that would render electrical equipment inoperable or unreliable, and the availability of a reliable source of replacement supplies such as masks, harnesses, and filters. A Soldier that requires nasal CPAP should not be deployed if these factors cannot be assured and the absence of nasal CPAP would hinder the Soldier from performing his/her military duties.

(7) Musculoskeletal. Soldiers with any recent musculoskeletal injury or surgery that prevents necessary mobility or firing a weapon should not deploy. Any chronic condition that restricts performance in the Soldier's MOS (for example, low back pain that prevents lifting) should be referred to an MEB/PEB (or processed under paras 9-10 and 10-26). If found fit for duty, the Soldier may be deployed unless he/she cannot function in the specific environment in which he/she is being assigned. Soldiers who meet medical retention standards but have a 3 in the profile cannot be deployed unless cleared by an MMRB.

(8) Psychiatric. (See ASD (HA) Policy Guidance for Deployment-Limiting Psychiatric Conditions and Medications for further information.)

(a) A psychiatric condition controlled by medication should not automatically lead to non-deployment. Soldiers with a psychiatric disorder in remission or whose residual symptoms do not impair duty performance may be considered for deployment duties. The commander makes the ultimate decision to deploy after consulting with the treating physician or other privileged provider. The availability, accessibility, and practicality of a course of treatment or continuation of treatment in theater or austere environment should be consistent with clinical practice standards. If there are any questions on the safety of psychiatric medication, a psychiatrist must be consulted.

(b) Psychotic and bipolar disorders are considered disqualifying for deployment.

(c) Psychiatric disorders that meet medical retention standards must demonstrate a pattern of stability without significant symptoms for at least 3 months prior to deployment.

(d) Soldiers must demonstrate behavioral stability and minimal potential for deterioration or recurrence of symptoms in a deployed, austere environment, to the extent this can be predicted. The potential for deterioration must be evaluated considering potential environmental demands and individual vulnerabilities.

(e) Medication disqualifying for deployment include-

1. Antipsychotics used to control psychotic, bipolar, and chronic insomnia symptoms; lithium and anticonvulsants to control bipolar symptoms;

2. Medications that require special storage considerations, for example, refrigeration;

3. Medications that require laboratory monitoring or special assessments, including lithium, anticonvulsants, and antipsychotics;

4. Medication prescribed within 3 months prior to deployment that has yet to demonstrate efficacy or be free of significant impairing side effects.

(f) Psychotropics clinically and operationally problematic during deployments include short half-life benzodiazepines and stimulants. Decisions to deploy personnel on such medications should be balanced with necessity for such medication in order to effectively function in a deployed setting, susceptibility to withdrawal symptoms, ability to secure and procure controlled medications, and potential for medication abuse.

(9) History of exertional heat illnesses. See paragraph 3-45 for profile guidance and MEB/PEB criteria. If there is any evidence of significant heat intolerance, the Soldier should not deploy to warm austere climates.

(10) Pregnancy. Pregnant Soldiers will not deploy.

(11) History of cancer. Soldiers with a history of cancer who have been returned to duty but have a requirement for periodic monitoring every 6 months or less should not deploy.

(12) Chronic infectious diseases (HIV, Hepatitis B or C). Soldiers will not deploy into the combat theater of operations. If found fit by a PEB, Soldiers may deploy to Europe or Korea (Host Nation permitting).

(13) Abnormal cervical cytology. Soldiers with recently treated moderate or severe dysplasia may only be deployed to austere environments if coordination is arranged via the unit commander and theater surgeon to ensure follow-up evaluation 7 to 9 months after initial evaluation and treatment.

(14) Malignant hyperthermia. Soldiers with history of malignant hyperthermia should not be assigned to areas where complete anesthesia services are unavailable or to austere environments. 
(15) Contact lenses. Soldiers who must wear contact lenses to achieve vision standards and who cannot satisfactorily perform their MOS with their best spectacle correction or fall below vision retention standards with their best spectacle prescription should not deploy. Contact lens wear is not authorized in field environments or while deployed.

(16) Miscellaneous conditions. Soldiers pending and scheduled for required surgeries or still in rehabilitation post surgery with temporary profiles, pain syndromes requiring the use of medications (beyond simple NSAIDS), or the use of transcutaneous electrical nerve stimulator units, should not deploy.

(17) Medications. Soldiers taking medications should not automatically be disqualified for any duty assignment. Medications used for serious and/or complex medical conditions are not usually suitable for extended deployments. The medications on the list below are most likely to be used for serious and/or complex medical conditions that could likely result in adverse health consequences. This is not an all-inclusive listing of medications that may render an individual non-deployable but is provided as a guideline to be used during pre-deployment medical screening. Because some medications are used for multiple reasons, any medical screening should take into account whether the drug is being used for a serious and/or complex medical condition or another use that might be appropriate for a deploying Soldier. A complete medical evaluation should be initiated on those personnel regularly taking the following medications:

- Antiarrhythmics.

- Antiasthmatics (long acting beta-agonists and inhaled corticosteroids only).

- Anti-cancer/chemotherapy agents.

- Anticoagulants (for example, heparin, warfarin).

- Anticonvulsants (for the treatment of seizure disorder).

- Antidepressants (moderate to severe cases, bipolar, or unstable patients).

- Anti-gout medications (for example, allopurinol, colchicines).

- Anti-HIV medications.

- Antimania (lithium).

- Antiparkinsonians.

- Antipsychotics.

- Antithyroid medications (propylthiouracil (PTU), methimozole).

- Anti-tumor necrosis factor (for example, enteracept, and so on).

- Any injectable medications.

- Any type of insulin.

- Any type of medication (oral or injected) used for the purpose of treating diabetes.

- Chronic anxiolytics.

- Chronic immunosuppressants (for example, azathioprine, cyclosporin, and so on).

- Chronic narcotic analgesics.

- Coronary vasodilators (nitrates).

- Heart failure medications.

- Hematologics (for example, EPO, G-CSF, GM-CSF, and so on).

- Immunomodulators (for example, interferons, methotrexate, and so on).

- Inflammatory bowel disease medications (for example, mesalamine, sulfasalazine, and so on).

- Metformin (see para 5-14e(2)).

(18) Dental. Soldiers that are in Dental Fitness Classification (DFC) 3 or 4 should not automatically be disqualified for any duty assignment. DFC 3 or 4 Soldiers should receive the necessary dental exam/treatment to make them at least a DFC 2 and thus deployable. Certain geographical areas with specific operational demands and limited access to care may require that the Soldier be in DFC 1 prior to deployment.

g. Medical Standards for Military Assistance Advisory Groups (MAAGs), military attaches, military missions, and duty in isolated areas where adequate medical care may not be available are listed below in paragraphs 5-14f(1)-(5). (See AR 55-46, AR 614-200, and AR 600-8-101.) These fitness standards also pertain to dependents of personnel being considered. The following medical conditions and defects will preclude assignments or attachment to duty with MAAGs, military attaches, military missions, or any type of duty in OCONUS isolated areas where adequate medical care is not available:

(1) A history of emotional or mental disorders, including character disorders, of such a degree as to have interfered significantly with adjustment or are likely to require treatment during this tour.

(2) Any medical conditions where maintenance medication is of such toxicity as to require frequent clinical and laboratory follow up or where the medical condition requires frequent follow up that cannot be delayed for the extent of the tour.

(3) Inherent, latent, or incipient medical or dental conditions that are likely to be aggravated by the climate or general living environment prevailing in the area where the Soldier is expected to reside, to such a degree as to preclude acceptable performance of duty.

(4) Of special consideration are Soldiers with a history of chronic cardiovascular, respiratory, or nervous system disorders who are scheduled for assignment and/or residence in an area 6,000 feet or more above sea level. While such individuals may be completely asymptomatic at the time of examination, hypoxia due to residence at high altitude may 
aggravate the condition and result in further progression of the disease. Examples of areas where altitude is an important consideration are La Paz, Bolivia; Quito, Ecuador; Bogota, Columbia; and Addis Ababa, Ethiopia.

(5) Remediable medical, dental, or physical conditions or defects that might reasonably be expected to require care during a normal tour of duty in the assigned area are to be corrected prior to departure from CONUS.

\section{5-15. Height-U.S. Military Academy, Reserve Officers-Training Corps, and Uniformed Services University of Health Sciences}

The following applies to all candidates to the USMA, the ROTC, and the USUHS. Candidates for admission to the USMA, the ROTC, and the USUHS who are over the maximum height or below the minimum height will automatically be recommended by DODMERB for consideration for an administrative waiver by HQDA during the processing of their cases.

\section{Chapter 6 \\ Aeromedical Administration}

\section{6-1. General}

a. This chapter provides-

(1) Administrative policies for completing the Army flying duty medical examination (FDME) and the flying duty health screen (FDHS).

(2) General policies for the review and disposition of aeromedically disqualified aviation training program applicants, aircrew, and ATCs.

$b$. The FDME is a periodic physical examination performed for occupational and preventive medicine purposes to promote and preserve the fitness, deployability, and safety of aviation personnel and resources. The FDME is a screening examination used as a starting point for the careful evaluation and treatment of aircrew member health problems. The FDME focuses on the history, vision, hearing, and cardiopulmonary and neuropsychiatric systems. The FDME and supporting documents provide the aviation commander and Director, U.S. Army Aeromedial Activity (USAAMA) with information to make a final determination of medical fitness for flying and ATC duties.

$c$. The FDHS is an interim health screening evaluation, done annually, between comprehensive FDMEs to ensure maintenance of aircrew health and fitness for aviation duty and serves an opportunity for health promotion and annual PHA. (See ATB, Administrative Guide.)

\section{6-2. Definition of terms}

a. AR 600-105 and AR 600-106 provide additional definitions and policies pertaining to aviation duties.

$b$. The terms aircrew duties, ATC duties, aviation service, flying status, flight status, and flying duty are essentially interchangeable.

$c$. The terms aircrew and aircrew member are interchangeable. They are personnel who are in or graduated from aviation or ATC training programs. (See paras 4-1 and 4-2.)

$d$. Aeromedical standard of care is the minimum level by which a FS/APA/AMNP/AME conducts a comprehensive aviation medicine program to conserve aircrew health maintenance, flight safety, and operational readiness. The basis of the standard is promulgated by TSG through regulations, APLs, and ATBs.

$e$. Aviation training programs are military courses of instruction that prepare personnel to perform rated or nonrated flying duties or ATC duties.

$f$. A U.S. military FS is a physician awarded the aeronautical designation of FS after graduation from a basic course in U.S. military aviation medicine.

g. An aerospace medicine specialist is a FS who successfully completed a residency in aerospace medicine, or equivalent as determined by the American Board of Preventive Medicine or TSG.

$h$. An aeromedical physician assistant (APA) is a physician assistant who successfully completed the U.S. Army Flight Surgeon Primary Course. An aviation medicine nurse practitioner (AMNP) is a family nurse practitioner who successfully completed the U.S. Army Flight Surgeon Primary Course. An aeromedical examiner (AME) is a physician who has had sufficient aeromedical training to allow him/her to independently conduct FDMEs/FDHSs, write aeromedical summaries, and issue DA Form 4186. The aeromedical training must be validated and approved by the Dean, USASAM and the Director, USAAMA.

$i$. The ACAP is a panel of senior Fort Rucker rated aviators designated by the Commander, U.S. Army Aviation Center, and RAMs/FSs with multiple medical specialty credentials designated by the Director, USAAMA, to include representatives from the U.S. Army Combat Readiness Center, the U.S. Army School of Aviation Medicine, and the U.S. Army Aeromedical Research Laboratory.

j. An Aeromedical Summary is a medical evaluation containing medical history, physical, and supportive materials 
prepared by a FS/APA/AMNP/AME and forwarded to USAAMA for making a final determination of medical fitness for flying duties.

$k$. Aeromedical disqualification (DQ) is a medical condition that is unfitting for aviation or ATC duties as prescribed in chapters 2 and 4. AR 600-105 contains definitions and procedures for temporary medical suspension, medical termination of aviation service, aeromedical waivers, and return to aviation service after termination of aviation service. AR 600-105 defines procedures for nonmedical disqualifications for aviation service, FEBs, and in-flight aeromedical evaluations.

l. Temporary aeromedical DQ is a failure to meet a standard of medical fitness for flying duties due to a minor, selflimited condition that is likely to resolve and result in re-qualification within 365 days. A temporary aeromedical DQ will become a permanent aeromedical DQ if the DQ condition persists for more than 365 days.

$m$. Permanent aeromedical DQ is a failure to meet a standard of medical fitness for flying duties due to a condition that will either require a waiver for continuation of aviation service or result in medical termination of aviation service.

$n$. Full flying duties (FFD) is a recommendation of medical fitness permitting flying or ATC duties as annotated by a FS/APA/AMNP/AME on DA Form 4186 (Medical Recommendation for Flying Duty).

$o$. The DNIF is a recommendation of medical unfitness prohibiting flying or ATC duties as annotated by a FS/APA/ AMNP/AME or other health care professional on DA Form 4186.

$p$. Date of medical incapacitation is the date a disqualifying medical condition was definitively diagnosed by history, examination, or test. The effective date of medical termination from aviation service is based on this date. This date may not always correspond with the date of DNIF issued by the local FS/APA/AMNP/AME on DA Form 4186.

$q$. Temporary flying duty clearance pending receipt of waiver may be granted following the guidance in APLs for certain conditions.

$r$. Aeromedical Epidemiology Resource Office (AERO) is the electronic, online paperless physical exam system that should be utilized for all Army Flight Duty Medical Examinations (FDME), interim FDME (also known as the Flight Duty Health Screens (FDHS)) and aeromedical summaries (AMS).

\section{6-3. Application}

The provisions of this chapter apply to FDMEs and Aeromedical Summaries accomplished for aircrew performing aviation or ATC duties in DA aircraft, aircraft leased by the DA, or in Army ATC facilities. This includes Active Army and RC personnel, to include ARNG/ARNGUS, DACs, and contract civilians under employment by the DA or firms under contract to the DA as per Chapter 4.

\section{6-4. Army Aviation Medicine Program responsibilities}

a. TSG is responsible for the Army Aviation Medicine Program. (See AR 40-3.)

$b$. The AMC to TSG-

(1) Provides recommendations on the recruitment, selection, utilization and assignment of FSs, APAs, AMNPs, and aerospace medicine specialists.

(2) In coordination with the Director, USAAMA, develops aeromedical policy and standards for aircrew selection, retention, operational effectiveness, and safety.

(3) In coordination with USAAMA, recommends medical fitness policy and standards for Army aircrew members to TSG.

(4) Develops memoranda of understanding between the Chief, Aviation Branch; Commander, AHRC; Chief, NGB; Commander, USAMEDCOM; and TSG as required.

c. The Director, USAAMA, supervises the Activity, the Aeromedical Consultative Service (ACS), and the ACAP, and maintains the Aviation Epidemiology Data Register (AEDR).

$d$. The Director, USAAMA, coordinates with the AMC to TSG, and aviation waiver authorities and-

(1) Implements and monitors aeromedical policy and standards for aircrew selection, retention, operational effectiveness, and safety.

(2) Develops a consensus of opinion on the final aeromedical recommendation of flying duties fitness for aircrew training applicants and trained aircrew members through the aeromedical review process. (See paras 6-6 through 6-20.)

(3) Monitors the quality and implementation of the FDME/FDHS program.

(4) Manages the ACAP, the ACS, and the Aircrew Epidemiology Branch.

(5) Monitors and manages the AERO system.

$e$. The ACAP provides consultation and opinions on selected issues and aeromedical board cases pertaining to aeromedical policy, standards, and fitness for flying duties. (See para 6-2i.)

$f$. The Chief, ACS reviews FDMEs, FDHSs, aeromedical board summaries, and organizes tertiary aeromedical consultation and in-flight evaluations of disqualified aircrew members. Selected and eligible aircrew members may be referred to the tertiary aeromedical consultative services of the U.S. Air Force, U.S. Navy, and Allied Nations when approved by the authorities of those services. Requests for tertiary aeromedical consultation are forwarded through the 
local FS/APA/AMNP/AME to Director, U.S. Army Aeromedical Activity (MCXY-AER), Fort Rucker, AL 36362-5333, (334) 255-7340. (See AR 600-105.)

g. The Chief, Aircrew Epidemiology Branch, manages the AEDR. The AEDR is a DA-directed aeromedical database for Army aircrew. As directed by TSG, the AEDR is established and maintained by USAAMA.

$h$. The Dean, U.S. Army School of Aviation Medicine-

(1) Manages the aeromedical policy and standards education of FSs, APAs, AMNPs, AMEs, flight medical aidmen, aeromedical evacuation aviators, and other health care providers supporting the aviation medicine program.

(2) Provides verification of aeromedical policy and standards compliance in the local aviation medicine clinic through the Aviation Resource Management Survey inspection program.

(3) Manages aeromedical physiologic education and training of aircrew members.

$i$. Directors of health services, MTF commanders, command surgeons, and aviation unit commanders implement the Army Aviation Medicine Program at the local level by providing trained personnel, equipment, and facilities for the proper conduct of the program. They ensure expeditious, accurate completion of FDMEs and aeromedical summaries by military and civilian FSs, APAs, AMNPs, and AMEs.

j. Local FS/APA/AMNP/AME-

(1) Provide clinical and preventive medicine care to aircrew members and airfield support personnel. Provide area support for the aviation medicine care of Army RC, ARNG/ARNGUS, ROTC, and Army Recruiting units (See AR 40-3, chapter 3).

(2) Manage the aeromedical certification of aircrew and ATC by issuance of DA Form 4186, periodic aviation medicine examinations, in-flight evaluations, and aeromedical summaries.

(3) Assist commanders in providing aircrew physiologic and survival training as specified in FM 3-04.301.

(4) Serve as aviation unit staff officers and members of mishap investigation, aviation safety, and FEBs as per AR 600-105, AR 385-10, and DA Pam 385-90.

(5) Develop, implement, and exercise the medical portion of the airfield accident response plan and unit operations, mission, and deployment plans.

(6) Conduct flight line inspections of aviation life support equipment and crash protection systems.

(7) Participate in all aspects of the unit flight mission as per AR 600-105.

\section{6-5. Authorizations}

a. The AMC to TSG is the proponent office for chapters 4 and 6 .

$b$. The AMC to TSG, in coordination with the USAAMA, issues APLs and ATBs to administer chapters 4 and 6.

\section{6-6. Classification of flying duty medical exams}

Paragraph 4-2 outlines the medical standards classification for flying duties. SF 88 and SF 93 have been replaced by DD Form 2808 (Report of Medical Examination) and DD Form 2807-1 (Report of Medical History).

\section{6-7. Purpose of flying duty medical exams}

a. Purpose categories. The FDME purpose is recorded with the FDME classification in Item 15c of DD Form 2808. The FDME/FDHS process accomplishes the requirements for the Annual PHA. There are four purpose categories for FDMEs:

(1) Initial FDME. Initial FDMEs are performed on all Class 1 aviator training program applicants; and all other Classes applying for or awaiting initial aviation or aviation medicine training, inter-service transfer, transition from active duty to RC, after being out of the military for more than 4 years, or hiring into the DAC, or DA contract civilian aircrew work force. The results of Initial FDMEs are recorded on DD Form 2807-1, DD Form 2808, and on aeromedical continuation sheets.

(2) Fort Rucker Class 1 FDME. Class 1 aviator training program students must have a valid, approved Initial Class 1 FDME before acceptance into aviator training programs and upon arrival for flight training at Fort Rucker. Lyster Army Health Clinic (LAHC) will perform a Fort Rucker Class 1 FDME before the student is enrolled in flight training to revalidate that the student meets Class 1 medical standards of fitness for flying duties. A repeat Initial FDME will be performed if the Initial FDME is no longer valid. The results of the Fort Rucker FDME are recorded on DD Form 2808, DD Form 2807-1, and associated aeromedical continuation forms; and if baseline medical history verification sheet from USAAMA is not available, USAAMA will determine a final recommendation.

(3) Comprehensive FDME. Comprehensive FDME. Comprehensive FDMEs are performed on all Classes of aircrew when Initial FDME or Interim FDHS are not required. (See para 6-8b, below.) The results of the Comprehensive FDME are recorded on DD Form 2808 and DD Form 2807-1. Report interim changes in medical history on DD Form 2807-1 if these changes were not previously documented on an AEDR Medical History Verification Report or Aeromedical Summary.

(4) Interim FDME. Interim FDHSs are performed on all Classes of aircrew when an Initial FDME or Comprehensive FDME is not required. (See para 6-8b, below.) The results of the Interim FDHS are recorded on DA Form 4497 (Interim (Abbreviated) Flying Duty Medical Examination) or DD Form 2808 with identified blocks specific for interim 
FDHS completion. Report interim changes in medical history on DD Form 2807-1 if these changes were not previously documented on an AEDR Medical History Verification Report or Aeromedical Summary.

$b$. Guidelines. Refer to ATB 2, Army Flight Surgeon's Administrative Guide, for guidelines on completing each category of examination.

\section{6-8. Frequency and period of validity of FDMEs}

a. Class 1 validity is as follows:

(1) Initial Class 1 FDME. The Initial FDME is valid for a period of 18 months from the date of examination. Repeat Initial FDMEs are required if the FDME validity expires while awaiting aviator training program selection or training class dates. The FDME must be valid and qualified by the Director, USAAMA, before the applicant's acceptance into aviator training programs and upon arrival for flight training.

(2) Fort Rucker Class 1 FDME. This physical is valid for up to 24 months to allow completion of the Flight Training programs. Upon graduation and PCS to the next duty station, the aviator will require completion of a FDME/ FDHS and birth month realignment, as prescribed in ATB 2 and table 6-1.

$b$. Classes $2 / 2 \mathrm{~F} / 3 / 4$ validity is as follows:

(1) Initial FDME. The Initial FDME is valid for a period of 18 months from the date of examination. Following the Initial FDME, subsequent Comprehensive FDMEs or Interim FDHSs will be aligned with the aircrew member's birth month using table 6-1.

(2) Comprehensive FDME. The Comprehensive FDME is performed every 5 years beginning with age 20, or as applicable, at ages $25,30,35,40$, and 45 . Beginning with age 50, a comprehensive FDME is required annually. It will be performed within 90 days before the end of the birth month in the year it is due. The FDME is valid until the end of the next birth month. Comprehensive FDME shall be completed and submitted after any Class A and B mishap.

(3) Interim FDME. The Interim FDHS is performed in the interim years when an Initial or Comprehensive FDME is not required. It will be performed within 90 days before the end of the birth month and is valid until the end of the next birth month. If retiring, the period of validity will extend to 18 months past the birth month.

(4) Rated aviators in aviation service. Rated aviators in aviation service are required to maintain a Comprehensive or Interim Class 2 FDME even when not assigned to operational flying duty positions. (See AR 600-105.)

(5) Additional comprehensive FDMEs. These may be required following disqualifying illness or injury present for more than 12 months, post mishap investigation, or FEB. A comprehensive FDME is required for those who are terminated from aviation service and are requesting a return to aviation service.

(6) Retirement. If an FDME is required within 90 days of retirement from Federal service, a comprehensive FDME with the additional examination requirements for retirement (see chap 8) is required for active duty members, and is encouraged but not required for $\mathrm{RC}$ or civilian members.

c. The requirement to perform FDMEs will not be suspended in the event of training exercises or military mobilization unless authorized by TSG. Request authorization through the Director, USAAMA (MCXY-AER), Fort Rucker, AL 36362-5333, who will coordinate authorization with the AMC to TSG.

$d$. The FDME will be completed to the extent the MTFs permit when aircrew are on duty or in mobilization at a station OCONUS with limited military medical facilities. Email or attach a cover letter to the FDME addressed to Director, USAAMA (MCXY-AER), explaining the facility limitations. Accomplish the missing portions of the annual FDME/FDHS within 90 days upon return to a station with adequate medical facilities. Align subsequent Comprehensive or Interim FDHSs with the aircrew member's birth month using table 6-1.

$e$. During certain missions not supported by U.S. or allied military medical officers (for example, special operations), the FDME may be deferred by the Commander having custody of the field personnel files until the accomplishment of the FDME becomes feasible. Annotate the remarks section of DA Form 4186 with an explanation of the deferment.

\section{6-9. Facilities and examiners}

a. U.S. military or civilian FS/APA/AMNP/AMEs at MTFs will conduct initial FDMEs. Initial FDMEs will meet the Army-specific administrative requirements for the completion of such FDMEs as outlined in ATB 2, Army Flight Surgeon's Administrative Guide. The FS/APA/AMNP/AME will apply U.S. Army aeromedical standards from chapters 2 and 4 for the determination of medical fitness for flying duty.

$b$. Comprehensive FDMEs and Interim FDHSs for all Classes will be conducted when possible by trained FS/APA/ AMNP/AMEs. The FDME may be conducted by any military, DAC, contract civilian physician, or non-aeromedically trained PA or FNP when a FS/APA/AMNP/AME is not available, but only a FS shall review and sign the DD Form 2808 and DD Form 2807-1 or DA Form 4497 prior to sending by mail or AERO the FDME to USAAMA for central review. When a FDME is performed at non-U.S. Army medical facilities, the FDME will be conducted by a military FS/APA/AMNP/AME to meet the administrative requirements of that branch of the U.S. Armed Forces or host Allied nation in accordance with STANAG 3526 and AR 12-15. APL, Aeromedical Cardiovascular Screening Program, still applies. The FS/APA/AMNP/AME must apply Army aeromedical standards from chapters 2 and 4 for the determination of medical fitness for flying duties. FDMEs performed by host Allied nations may be completed in English on 
Allied documents designed for the same purpose when DD Form 2808 and DD Form 2807-1 are not available. Outline unusual circumstances in a memorandum for record included with the FDME.

c. The DAC or DA contract civilian providers with a previous military aeronautical rating of FS/APA/AMNP/AME practicing in medical specialties other than aviation medicine may be credentialed to complete FDMEs. The U.S. Army School of Aviation Medicine provides Army Aviation Medicine refresher training for FSs/APAs/AMNPs/AMEs to meet credentialing requirements. Other physicians and health care professionals will sign the DD Form 2808 for the portions of the examination they accomplish. The FDME is invalid and incomplete without the signature of a military FS/APA/AMNP/AME on the DD Form 2808 and DD Form 2807-1 or DA Form 4497, and a final review stamp placed by the staff of USAAMA on the DD Form 2808 or DA Form 4497.

d. The APAs and AMNPs may conduct FDMEs/FDHSs and sign/submit normal FDMEs/FDHSs meeting aeromedical class standards and those with waivers meeting annual waiver requirements directly to USAAMA for review and disposition. All others (that is, new disqualifications or not meeting annual waiver requirements) must be reviewed and co-signed by the supervising flight surgeon for submission. In circumstances when supervising flight surgeon is unavailable, APAs/AMNPs shall annotate such on DD Form 2808, Block 73, "Notes" for USAAMA flight surgeon staff to assume that role. LAHC APAs and AMNPs may submit all FDMEs, FDHSs, and AMSs directly to USAAMA.

$e$. Consultations may be obtained at Government expense when authorized as stated below. (See also paras 4-3 and 4-32.)

(1) Additional tests, procedures, and consultations required to complete Initial FDMEs for all aircrew Classes, to include civilians, active duty, and RC, will be completed at military outpatient or inpatient MTFs. When fitness for flying duty cannot be determined, MTF commanders or ARNG/ARNGUS State Adjutant General's Office may permit supplementary examinations from civilian medical sources. The tests and consultations are conducted only to the extent required to determine medical fitness for flying duties and not for the treatment or correction of disqualifying conditions.

(2) Paragraph (1), above, applies to Comprehensive FDMEs and annual Interim FDHSs, except that treatment or correction of disqualifying conditions discovered during the FDME/FDHS will be completed if the examinee is eligible for such care (AR 40-400).

(3) The DACs or contract civilians utilizing Army flight standards and employed by DA or firms under contract by DA who are military retirees, RC, or ARNG/ARNGUS aircrew, may be authorized for such care. (See (1) and (2) above, and AR 40-400.)

(4) The DAC or contract civilian utilizing Army flight standards and may request a waiver of the disqualifying condition from the Director, USAAMA. The Director, USAAMA, will process any waiver request consistent with guidance for granting waivers.

(5) Director, USAAMA, may direct evaluation of disqualified aircrew eligible for care (AR 40-400) at any U.S. MTF or aeromedical consultation service.

$f$. The AERO (online system) is the primary method for entering and submitting FDME and AMS data.

\section{6-10. Disposition and review of flying duty medical examinations}

a. Review. The review of the individual health record and FDME/FDHS will be completed by the aeromedical health care provider. The aeromedical health care provider will counsel the examinee regarding-

(1) Conditions found during the FDME.

(2) Continuing care for conditions under treatment and/or waiver.

(3) General preventive health education, including, but not limited to smoking, cholesterol control, weight control, drug and alcohol abuse, and other high risk behavior.

b. Profile status. The examinee's current PULHES profile status shall be recorded in the PULHES section of the DD Form 2808. See APL, Unspecified Permanent Profiles (Ortho Chapter).

c. Class 1 and initial Classes 2/2F/4. Completed FDMEs (originals of DD Form 2807-1, DD Form 2808, aeromedical continuation sheet, interpreted EKG, and other supportive documents) accomplished for application to aviation and aviation medicine training programs will be forwarded through the procurement chain of command of the applicant via AERO preferred, or by mail as an alternate, to Director, USAAMA (MCXY-AER), Fort Rucker, AL 36362-5333 for central aeromedical review and disposition. The FS's office shall place copy of the FDME and all enclosures in the medical record and retain a copy in the office for a minimum of 2 years if submission is not accomplished via AERO. The AERO serves as a repository for the information. In no case will the originals be given to the applicant or other individuals not in the procurement chain of command. The Director, USAAMA, must make a final determination of fitness for flying duties before Classes 1/2F/4 applicants may be accepted and assigned to Fort Rucker for aviation, ATC, and aviation medicine training programs.

d. Trained Classes 2/2F/4. Completed Comprehensive and Interim FDHSs (DD Form 2808 and DD Form 2807-1 or DA 4497-R, aeromedical continuation forms, interpreted EKG, and other supportive documents, may include consultations, EKG tracings, radiographs, coronary angiogram, and so forth, and, if applicable, Aeromedical Summary) will be forwarded directly to Director, USAAMA, ATTN: MCXY-AER, Fort Rucker, AL 36362-5333, for central aeromedical review and disposition. The FS's office shall place copy of the FDME/FDHS and all enclosures in the medical 
record and retain a copy in the office for a minimum of 2 years if submission is not accomplished via AERO. AERO serves as a repository for the information.

e. Class 3. The attending FS/APA/AMNP/AME who signs the FDME/FDHS is the reviewing authority for recommending disposition on medical fitness for flying duty. Minor medical disqualifications that will in no way affect the safe and efficient performance of flying duties and that will not be aggravated by aviation duties or deployment may be waived by the individual's unit commander upon favorable recommendation by the attending FS/APA/AMNP/AME. (See also APL, Class 3 Aircrew, and para 4-33.) (See also ATB 2, Army Flight Surgeon's Administrative Guide, for details on the item-by-item completion of FDMEs.) AERO should be utilized for completion of Class 3 FDME/FDHS and AMS for the purpose of annotating and archiving aeromedical information in the AEDR repository.

f. Tracking. The flight surgeon or aviation unit will track FDMEs/FDHSs via AERO from initiation until posted in the health record with a final disposition by USAAMA. If disqualified, the flight surgeon and aviation unit will take action as per AR 600-105 and AR 600-106.

g. Disposal of documentation. Waiver and suspension recommendation and approval letters will be filed in the individual health record and flight record. When available, the AERO Abbreviated Waiver Letter shall be utilized for the individual flight record folder (IFRF) for Health Insurance Portability and Accountability Act (HIPAA) compliance of protected health information.

\section{6-11. Issuing DA Form 4186}

$a$. DA Form 4186 is an official document used to notify the aviation commander of certification of medical fitness for all Classes of military and civilian aircrew utilizing Army standards for medical clearance.

$b$. The DA Form 4186 will be completed-

(1) After the completion of an FDME/FDHS.

(2) After an aircraft mishap.

(3) After an FEB.

(4) When reporting to a new duty station or upon being assigned to operational flying duty.

(5) When admitted to and discharged from any medical or dental treatment facility (inpatient or outpatient, military or civilian), sick in quarters, interviewed for or entered into a drug/alcohol treatment program, or when treated by a health care professional who is not a military FS/APA/AMNP/AME or otherwise authorized to issue a DA Form 4186.

(6) When treated as an outpatient for conditions or with drugs that do not meet the standard for aviation duties; and upon return to flight duties after such treatment and recovery.

(7) Upon return to flight status after termination of temporary medical suspension, issuance of waiver for aviation service, or requalification after medical or nonmedical termination of aviation service.

(8) Other occasions as required by the FS/APA/AMNP/AME.

c. Rated aviators not performing operational flying duties are required to complete an annual FDME/FDHS with issuance of DA Form 4186 (AR 600-105).

$d$. Each item of the DA Form 4186 will be completed as directed by the Director, USAAMA. (See ATB, DA Form 4186.) Three copies of the DA Form 4186 will be completed. Copy 1 is placed in the outpatient medical record. Copy 2 is forwarded to the examinee's unit commander who signs and forwards it to the flight operations officer for inclusion in the flight records (AR 95-1 and FM 3-04.300). Copy 3 is given to the examinee.

$e$. If the examinee is found qualified for flying duty by the local FS/ APA/AMNP/AME, see chapters 2 and 4. Issuance of the DA Form 4186 will constitute an aeromedical clearance for flying duty pending final review of the FDME/FDHS by the reviewing authority. The aeromedical clearance will expire when the current FDME/FDHS is no longer valid. (See para 6-8.)

$f$. If a disqualifying medical condition is found, a waiver must be granted by the appropriate authority before further flying duties are performed. (See paras 6-12 through 6-20.) For minor defects that will not preclude safe and efficient performance of flying duties and will not be aggravated by aviation duty or military mission, the local commander may permit an individual to continue performance of aviation duties pending completion of the formal waiver process and upon favorable recommendation for temporary FFD by the local FS/APA/AMNP/AME following the guidelines in APL, Temporary Flying Duties.

g. When used to recommend temporary flying duties, the Remarks section of DA Form 4186 will be completed to reflect a limited length of time for which the clearance is issued, for example: "Temporary FFD, 90 days, pending receipt of waiver."

$h$. The FS/APA/AMNP/AME will consult the Director, USAAMA (MCXY-AER), or the major Army command's Aviation Medicine consultants in U.S. Army, Europe or Korea, before issue of DA Form 4186 for complex or questionable cases.

$i$. The validity period of the current FDME/FDHS (see para 6-8) may be extended for a period of 1 calendar month beyond the birth month on the DA Form 4186. After expiration of this extension, the aircrew member or ATC must complete the FDME/FDHS and be medically qualified or be- 
(1) Administratively restricted from flying duties if no aeromedical DQ exists and be considered for a non-medical DQ and FEB (AR 600-105).

(2) Medically restricted from flying duties if an aeromedical DQ exists. In some cases, temporary flying duties may be recommended on DA Form 4186. (See also $f$, above, and paras 6-12 through 6-20.)

$j$. Personnel authorized to sign the DA Form 4186 are as follows:

(1) Any physician or health care provider may sign DA Form 4186 for the purpose of restricting aircrew and ATCs from aviation duties when an aeromedical DQ exists. (See $b$, above, and chap 4.)

(2) A FS/APA/AMNP/AME may sign the DA Form 4186 to return aircrew and ATCs to FFD. Recommended restrictions will be annotated in the Remarks block of DA Form 4186. (The previous requirement for an APA (and AMNP) to have the FS review the medical record and cosign within 72 hours is no longer required.)

(3) A non-aeromedically trained provider under the supervision of a FS may sign the DA Form 4186 to recommend returning aircrew and ATCs to FFD when a FS is not locally available by obtaining case-by-case telephonic guidance from a FS. The name of the consulted FS will be annotated on DA Form 4186, and on an SF 600 (Health RecordChronological Record of Medical Care) in the patient health record.

$k$. Forms of the other branches of the U.S. Armed Forces and host Allied nations similar to DA Form 4186 will be accepted by the Army when aeromedical support is provided by those Service/nations and DA Form 4186 is not available.

l. Contract aircrew utilizing FAA for aeromedical certification shall possess the applicable FAA Form 8500-9, Medical Certification, in lieu of DA Form 4186.

\section{6-12. General principles}

a. The Director, USAAMA, is authorized to issue APLs and ATBs that are regulatory in nature. These detail aeromedical policy and disposition for common aeromedical DQs and establish an Armywide standard of aeromedical care. These series may be obtained from Director, USAAMA (MCXY-AER), Fort Rucker, AL 36362-5333 or via the website at: https://aamaweb.usaama.rucker.amedd.army.mil/.

$b$. The FS/APA/AMNP/AME will make the initial determination of medical unfitness due to failure to meet a medical standard for-

(1) Aircrews. (See chaps 2 and 4, and AR 600-105.) The final determination of medical fitness for flying duties is made by the Director, USAAMA. Although MEB and PEB documents (AR 40-400 and AR 635-40) are valuable sources of information, the final recommendation of medical fitness for flying duty is made independent of the recommendations of these boards. The Director, USAAMA may review the proceedings of FEBs (AR 600-105) in determining fitness for flying duties.

(2) Personnel retention, retirement, or separation. (See chap 3.) The final determination of medical fitness for personnel retention, retirement, or separation is made by the MEB and PEB process (AR 635-40). In the case of aircrew members, the president of the PEB may request a consultation from the Director, USAAMA, or delay final determinations until the medical fitness for flying duties is determined by the Director, USAAMA.

c. The FS/APA/AMNP/AME will complete a history, physical, tests, and consultations to the extent required to-

(1) Confirm the medical disqualification.

(2) Recommend an aeromedical disposition.

(3) Meet the aeromedical standard of care in accordance with APLs and ATBs.

$d$. For all flying classes, each disqualifying defect or condition will be evaluated to determine if it-

(1) Is progressive.

(2) Is subject to aggravation by military Service.

(3) Precludes satisfactory completion of training and/or military service.

(4) Constitutes an undue hazard to the individual or to others.

e. The FS/APA/AMNP/AME will consider the factors involved in the use of medicines (APL, Medications) for treatment of the condition and determine if-

(1) The medication is effective without aeromedically significant side effects.

(2) There is a problem with medication compliance.

(3) The medication is readily available during mobilization.

(4) The medication does not mask symptoms subject to acute incapacitation or complications in the aviation environment.

$f$. The FS/APA/AMNP/AME will consider whether continued flying duty may-

(1) Compromise personal health.

(2) Pose a risk to aviation safety.

(3) Jeopardize mission completion.

(4) Result in deployability limitations.

g. The FS/APA/AMNP/AME will determine the date of medical incapacitation. The date of medical incapacitation is the date the aeromedical DQ is diagnosed by history, physical examination, or testing. The date of aeromedical 
incapacitation may not always correspond with the dates of local medical restriction from flying duties by an FS/APA/ AMNP/AME using DA Form 4186 or the date an FS/APA/AMNP/AME first evaluates the aeromedical DQ.

$h$. For the purpose of aeromedical DQs, the immediate aviation commander is defined as the aviation unit commander or designated official who maintains the aircrew member's flight or ATC records.

$i$. Each aeromedical DQ requires-

(1) Temporary medical suspension until the aircrew member is requalified and meets the medical standards of fitness for flying duties within 365 days (para 6-17); or

(2) Medical termination from aviation service (permanent medical suspension) due to a temporary medical suspension imposed for greater than 365 days or a permanent aeromedical DQ without waiver (para 6-18); or

(3) Aeromedical waiver granted by the aviation service waiver authority permitting aviation service despite an aeromedical DQ (para 6-19). (See ATB 3, Aeromedical Summary, for policy on the preparation of the Aeromedical Summary document, and ATB 4, Aeromedical Consultation Service, for policy on use of this service. See also ATB 2, Army Flight Surgeon's Administrative Guide.)

\section{6-13. Responsibilities and review following a change in health of aircrew members}

a. Aircrew members will report to a FS/APA/AMNP/AME the following conditions:

(1) Symptoms indicating a change in health.

(2) An illness requiring the use of medications, a visit to a health care provider for evaluation and/or medical-dental care, restriction to quarters, or hospitalization.

(3) Drug or alcohol use that results in legal problems (driving under the influence, driving while intoxicated, positive blood or urine drug screen, arrests for intoxication, family member abuse, and so forth), psychological dysfunction (absence or tardiness from work or school, severe marital discord, and so forth), medical or psychological incapacitation, or history of evaluation and/or treatment for drug/alcohol misuse, abuse, or dependence.

(4) Current aeromedical waivers or requests for waiver.

(5) Positive HIV.

$b$. Aircrew members must inform their FS/APA/AMNP/AME when they have participated in activities, or received treatment for which flying restrictions may be appropriate in accordance with AR 40-8. Treatment means any medical treatment or procedure performed by a non-aeromedical health care provider, and includes, but is not limited to, the following:

(1) Any medical or dental procedure requiring use of medications after treatment.

(2) Any medical or dental procedure requiring use of any type of anesthesia or sedation.

(3) Referral for psychological, social, or psychiatric counseling, to include any kind of alcohol or substance abuse counseling.

(4) Any chiropractic or osteopathic manipulative treatment.

(5) Any treatment given by a homeopath, naturopath, herbalist, acupuncturist, or other "alternative medicine" practitioner.

(6) Emergency room visits, or treatment given by a civilian health care provider.

c. The immediate aviation commander will request an aeromedical consultation with a local FS/APA/AMNP/AME when an aircrew member develops a change in health. (See a above.)

$d$. The local FS/APA/AMNP/AME will make a preliminary determination of medical fitness for flying duties and recommend FFD or DNIF by issuance of DA Form 4186. (See also paras 6-11 through 6-20.) Also, the attending FS/ APA/AMNP/AME will forward the FDME/FDHS via AERO with pertinent attachments or Aeromedical Summary to Director, USAAMA (MCXY-AER), Fort Rucker, AL 36362-5333 for review and final recommendation. See ATB 2, Army Flight Surgeon's Administrative Guide, and ATB 3, Aeromedical Summary. For rated flying personnel who have been found per\&\#172;manently disqualified for aviation service and for whom waivers are not being considered, Director, USAAMA (MCXY-AER) will notify the FAA. Authority is according to 5 USC 552a(b)7.

$e$. In the case of a permanent aeromedical disqualification (DQ), the Director, USAAMA (MCXY-AER), makes the final recommendation of medical fitness for flying duties to the aviation service waiver authority.

$f$. The aviation service waiver authority reviews the recommendation of medical fitness for flying duties and makes the final administrative disposition for-

(1) Medical termination from aviation service (permanent medical suspension); or

(2) Continuation of aviation service with administrative aeromedical waiver.

g. The aviation service waiver authorities are listed in paragraph 6-20.

$h$. The aeromedical consultation authority is Director, USAAMA, ATTN: MCXY-AER (Chief, Aeromedical Consultation Service), Fort Rucker, AL 36362-5333.

\section{6-14. Review and disposition of disqualifications for Class 3}

$a$. The FS/APA/AMNP/AME who signs the FDME is the reviewing authority and will make decisions on aeromedical disposition. Minor physical defects that will not affect the safe, efficient performance of flying duties or mission 
and will not be aggravated by aviation duties or deployment may be waived by the individual's unit commander, the Class 3 waiver authority, upon favorable recommendation by the FS/APA/AMNP/AME. (Exceptions are stated in paras 4-32 and $d$ below.)

$b$. Notification of aeromedical DQ will be forwarded on DA Form 4186 to the aviation unit commander, along with appropriate recommendations for waiver of DQs or suspension from flying duties in accordance with existing directives.

c. An Aeromedical Summary (AERO preferred) discussing the case and the basis for aeromedical decision will be prepared by the FS/APA/AMNP/AME and placed in the aircrew member's individual health record for future reference by the aviation commander and other FS/APA/AMNP/AMEs.

$d$. Cases involving drug/alcohol abuse or dependence, or complicated questionable cases shall be forwarded to Director, USAAMA (MCXY-AER), for review and disposition. (See also APL, Class 3, Aircrew.)

\section{6-15. Review and disposition of disqualifications for Classes $2 / 2 F / 4$}

a. Initial and periodic FDMEs will be submitted to Director, USAAMA, for review and disposition. (See para 6-10d.)

$b$. If the aircrew member is found medically qualified, the FS/APA/AMNP/AME prepares a DA Form 4186 and recommends clearance for FFD. (See para 6-11.)

c. If a disqualifying defect is discovered, the FS/APA/AMNP/AME completes the evaluation and recommends temporary medical suspension, termination from aviation service (permanent suspension), or waiver of the disqualifying defect. (See paras 6-17 through 6-20.)

\section{6-16. Temporary medical suspension}

a. A temporary medical suspension restricting aircrew from flying duties is required for temporary aeromedical DQs that are minor, self-limited, and likely to result in requalification within 365 days. Examples include ankle sprain, acute rhinitis, gastroenteritis, and simple closed fracture.

$b$. Medical termination from aviation service (see para 6-18) is mandatory if the temporary medical suspension exists for greater than 365 days (AR 600-105 and DOD 7000.14-R, Vol 7A). In this case, the temporary medical DQ becomes a permanent medical DQ.

c. The local FS/APA/AMNP/AME will evaluate all aircrew with possible aeromedical DQs as identified by the aviator, immediate commander, FS/APA/AMNP/AME, or USAAMA. The FS/APA/AMNP/AME will follow the established standards of aeromedical care (this regulation and APL and ATB series).

$d$. The FS/APA/AMNP/AME will recommend a date of medical incapacitation and recommend DNIF on DA Form 4186.

$e$. The immediate commander will set the date of medical incapacitation and impose the temporary medical suspension.

f. Aircrew under temporary medical suspension may not be assigned flying/ATC duties or operate the flight controls of a military aircraft. As an exception, the FS/APA/AMNP/AME may recommend by DA Form 4186 that the officer operate flight simulators, perform ground run-up procedures, and/or undergo an aeromedical consultation with in-flight evaluation. (See AR 600-105.)

$g$. The immediate commander may remove the temporary medical suspension upon favorable recommendation by a FS/APA/AMNP/AME on DA Form 4186.

$h$. The FS/APA/AMNP/AME will recommend medical termination from aviation service (permanent medical suspension) if the term of temporary medical suspension has or is expected to exceed 365 days. The FS/APA/AMNP/ AME will notify the immediate commander by DA Form 4186 and forward an Aeromedical Summary to Director, USAAMA, ATTN: MCXY-AER.

\section{6-17. Medical termination from aviation service}

a. Medical termination from aviation service (permanent medical suspension) is required for permanent aeromedical DQs that are not likely to result in requalification within 365 days. Continuation of flying duties is only authorized by issuance of orders for an aeromedical waiver (para 6-19) by an aviation service waiver authority.

$b$. The local FS/APA/AMNP/AME will evaluate the aeromedical DQ and make a preliminary determination of medical fitness for flying duty.

c. The FS/APA/AMNP/AME will recommend a medical termination from aviation service (permanent medical suspension) on DA Form 4186 and forward the notification to the immediate commander.

d. The FS/APA/AMNP/AME will prepare an Aeromedical Summary and forward to Director, USAAMA, ATTN: MCXY-AER.

$e$. The Director, USAAMA, ATTN: MCXY-AER will make final recommendations to the aviation service waiver authority and recommend a-

(1) Date of medical incapacitation.

(2) Final aeromedical disposition: 
(a) Medical termination from aviation service; or

(b) Aeromedical waiver for continuation of aviation service with the permanent aeromedical DQ; or

(c) Requalification without aeromedical DQ ("For Information Only").

$f$. The aviation service waiver authority will-

(1) Establish the date of medical incapacitation.

(2) Establish the date of medical termination from aviation service and publish an order (AR 600-8-105).

(3) Refer the aircrew member to the appropriate authority for reclassification, rebranching, or Service separation.

(4) Send the health record back to the MTF of origin.

g. The FAA Federal Air Surgeon requires the Director, USAAMA to report all termination from aviation service actions. This may be done without the knowledge or consent of the aircrew member (5 USC 552).

\section{6-18. Aeromedical waiver}

a. In the case of permanent aeromedical DQ, the aircrew member may request consideration for an aeromedical waiver for aviation service through a local FS/APA/AMNP/AME.

$b$. The FS/APA/AMNP/AME will complete an evaluation within the aeromedical standards of care (this regulation and APL and ATB series). The FS/APA/AMNP/AME will prepare an Aeromedical Summary and forward to Director, USAAMA (MCXY-AER).

c. The Chief, ACS will-

(1) Review the case.

(2) Arrange for additional evaluation by aeromedical consultants designated by Director, USAAMA as required.

(3) Authorize and arrange for additional evaluations at U.S. Air Force or U.S. Navy aeromedical consultation services as required.

(4) Arrange for in-flight evaluations as required (AR 600-105).

(5) Present selected cases to the ACAP.

(6) Refer the case with recommendations to Director, USAAMA, ATTN: MCXY-AER.

$d$. The Director, USAAMA will-

(1) Formulate a consensus of aeromedical opinion on the medical fitness for flying duty.

(2) Determine if an aeromedical waiver can be recommended, and if so, determine if the waiver will require recommendations for specific restrictions in the flight environment and/or specific followup medical evaluations to maintain the waiver.

$e$. The Director, USAAMA will forward final recommendations to the aviation waiver authority.

$f$. The aviation service waiver authority will-

(1) Review the aeromedical recommendations and supportive enclosures, consider the needs of the U.S. Army, and make a final determination to grant or deny an aeromedical waiver.

(2) Publish orders to permit continuation of aviation service with a waiver or medical termination from aviation service (permanent medical suspension).

(3) Send the health record back to the MTF of origin.

g. The aircrew member will acknowledge the waiver, and if applicable, restrictions and followup evaluation, in writing to the aviation service waiver authority. Failure to do so, or declining the waiver, will be considered a nonmedical DQ due to dereliction of duty and may result in an FEB (AR 600-105).

$h$. The FS/APA/AMNP/AME may recommend amendments to the conditions for continuation of waivers in effect, as required, by submitting written justification along with supportive documents to the Director, USAAMA (MCXY-AER), Fort Rucker, AL 36362-5333.

$i$. If the condition resolves or is no longer disqualifying due to policy and standard changes, the FS/APA/AMNP/ AME may recommend revocation of an aeromedical DQ to the Director, USAAMA.

\section{6-19. Aeromedical requalification}

a. An aircrew member with a medical termination from aviation service may request aeromedical requalification if the medical DQ resolves.

$b$. The procedure for requesting requalification is the same as the procedure for aeromedical waiver (para 6-19), except the aviation service waiver authority will determine if requalification meets the needs of the Army, and if so, will-

(1) Publish orders establishing date of the aeromedical requalification.

(2) Publish orders of assignment and travel.

(3) Issue an administrative waiver if required. 


\section{6-20. Waiver and suspension authorities}

a. Personnel who are dual-status (such as ARNG/ARNGUS members and DACs) will require a waiver or suspension action from each authority they are assigned.

b. Active Army, USAR, or International Military Pilots and Flight Students of non-NATO or Partnership for Peace (PfP) countries-Class 1 and Class 2: through Director, USAAMA (MCXY-AER), Fort Rucker, AL 36362-5333; for Commander, AHRC (TAPC-PLA), 200 Stovall Street, Hoffman Building, Room 3N25, Alexandria, VA 22332-0413.

c. Active Army or USAR-Class 2F/2P; and Class 3 aeromedical positions of aviation audiologists, dentists, optometrists, and psychologists requiring a central waiver (see APL, Class 3 aircrew): through Director, USAAMA (MCXY-AER), Fort Rucker, AL 36362-5333; for Commander, AHRC, Health Services Division (TAPC-OPH-MC), 200 Stovall Street, Hoffman Building, Room 9N68, Alexandria, VA 22332-0413.

d. Active Army or USAR-Class 3 (requiring a central waiver (see APL, Class 3 aircrew)), and Class 4: through Director, USAAMA (MCXY-AER), Fort Rucker, AL 36362-5333; for Commander, AHRC (TAPC-EPL-T), 2461 Eisenhower Avenue, Alexandria, VA 22331-0453.

e. ARNG/ARNGUS - Class 1, Classes 2/2F/4, and Class 3 (requiring a central waiver (see APL, Class 3 aircrew)): through Director, USAAMA (MCXY-AER), Fort Rucker, AL 36362-5333; for Chief, National Guard Bureau (NGB-AVN-OP), 111 South George Mason Drive, Arlington, VA 22204-1382.

f. Contract civilians - all Classes utilizing Army Flight Standards and not FAA for certification: through Director, USAAMA (MCXY-AER), Fort Rucker, AL 36362-5333; through the Contracting Officer Representative, for the Commanding General, or the Commanding General who is designated the waiver authority of the installation with the DA contract (usually the airfield commander or the command aviation officer of the installation with the DA contract; for example, at Fort Rucker, Command Aviation Officer (DPT-AD), Fort Rucker, AL 36362). Final determination will then be forwarded to the Contracting Office and the firm under contract to DA.

g. DAC-all Classes: through Director, USAAMA (MCXY-AER), Fort Rucker, AL 36362-5333; through aviation unit Commander; for the Commanding General or the Commanding General who is designated the waiver authority (usually the airfield commander or command aviation officer; for example, at Fort Rucker, Command Aviation Officer (DPT-AD), Fort Rucker, AL 36362). Final determination will then be forwarded to the local Civilian Personnel Office.

$h$. Class 3, for other than centrally required waivers and as specified in the APLs/ATBs: through the local FS/APA/ AMNP/AME; for the local aviation unit Commander.

Table 6-1

Number of months for which a flying duty medical examination (FDME) is valid (Active Component)*

\begin{tabular}{|c|c|c|c|c|c|c|c|c|c|c|c|c|}
\hline \multicolumn{13}{|c|}{ Month in which last FDME was given } \\
\hline Birth Month & Jan & Feb & Mar & Apr & May & Jun & Jul & Aug & Sep & Oct & Nov & Dec \\
\hline Jan & 12 & 11 & 10 & 9 & 8 & 7 & 18 & 17 & 16 & 15 & 14 & 13 \\
\hline Feb & 13 & 12 & 11 & 10 & 9 & 8 & 7 & 18 & 17 & 16 & 15 & 14 \\
\hline Mar & 14 & 13 & 12 & 11 & 10 & 9 & 8 & 7 & 18 & 17 & 16 & 15 \\
\hline Apr & 15 & 14 & 13 & 12 & 11 & 10 & 9 & 8 & 7 & 18 & 17 & 16 \\
\hline May & 16 & 15 & 14 & 13 & 12 & 11 & 10 & 9 & 8 & 7 & 18 & 17 \\
\hline Jun & 17 & 16 & 15 & 14 & 13 & 12 & 11 & 10 & 9 & 8 & 7 & 18 \\
\hline Jul & 18 & 17 & 16 & 15 & 14 & 13 & 12 & 11 & 10 & 9 & 8 & 7 \\
\hline Aug & 7 & 18 & 17 & 16 & 15 & 14 & 13 & 12 & 11 & 10 & 9 & 8 \\
\hline Sep & 8 & 7 & 18 & 17 & 16 & 15 & 14 & 13 & 12 & 11 & 10 & 9 \\
\hline Oct & 9 & 8 & 7 & 18 & 17 & 16 & 15 & 14 & 13 & 12 & 11 & 10 \\
\hline Nov & 10 & 9 & 8 & 7 & 18 & 17 & 16 & 15 & 14 & 13 & 12 & 11 \\
\hline Dec & 11 & 10 & 9 & 8 & 7 & 18 & 17 & 16 & 15 & 14 & 13 & 12 \\
\hline
\end{tabular}

Notes:

* Read down the left column to the examinee's birth month; read across to month of last FDME; intersection number is the maximum validity period. When last FDME was within the 3-month period preceding the end of the birth month, the validity period will normally not exceed 15 months. When the last FDME was for entry into aviation training, for FEB, postaccident, posthospitalization, pre-appointment (warrant officer candidate) etc., the validity period will range from 7 to 18 months. Validity periods may be extended, in accordance with 6-11i, by 1 month only for completion of an examination begun before the end of the birth month. 


\section{Chapter 7 \\ Physical Profiling}

\section{7-1. General}

This chapter prescribes a system for classifying individuals according to functional abilities. Also see paragraphs 3-12, $3-13,3-25,3-27,3-30,3-45$, and 3-46 for additional guidance on amputations, coronary artery disease, asthma, seizure disorders, and heat and cold injuries.

\section{7-2. Application}

The physical profile system is applicable to the following categories of personnel:

$a$. Registrants who undergo an induction or pre-induction medical examination related to Selective Service processing.

$b$. All applicants examined for enlistment, appointment, or induction.

c. Members of any component of the U.S. Army throughout their military Service, whether or not on active duty.

\section{7-3. Physical profile serial system}

$a$. The physical profile serial system is based primarily upon the function of body systems and their relation to military duties. The functions of the various organs, systems, and integral parts of the body are considered. Since the analysis of the individual's medical, physical, and mental status plays an important role in assignment and welfare, not only must the functional grading be executed with great care, but clear and accurate descriptions of medical, physical, and mental deviations from normal are essential.

$b$. In developing the system, the functions have been considered under six factors designated "P-U-L-H-E-S." Four numerical designations are used to reflect different levels of functional capacity. The basic purpose of the physical profile serial is to provide an index to overall functional capacity. Therefore, the functional capacity of a particular organ or system of the body, RATHER THAN THE DEFECT PER SE, will be evaluated in determining the numerical designation $1,2,3$, or 4 .

$c$. The factors to be considered are as follows:

(1) P-Physical capacity or stamina. This factor, general physical capacity, normally includes conditions of the heart; respiratory system; gastrointestinal system, genitourinary system; nervous system; allergic, endocrine, metabolic and nutritional diseases; diseases of the blood and blood forming tissues; dental conditions; diseases of the breast, and other organic defects and diseases that do not fall under other specific factors of the system.

(2) U-Upper extremities. This factor concerns the hands, arms, shoulder girdle, and upper spine (cervical, thoracic, and upper lumbar) in regard to strength, range of motion, and general efficiency.

(3) L-Lower extremities. This factor concerns the feet, legs, pelvic girdle, lower back musculature and lower spine (lower lumbar and sacral) in regard to strength, range of motion, and general efficiency.

(4) H-Hearing and ears. This factor concerns auditory acuity and disease and defects of the ear.

(5) E-Eyes. This factor concerns visual acuity and diseases and defects of the eye.

(6) S-Psychiatric. This factor concerns personality, emotional stability, and psychiatric diseases.

$d$. Four numerical designations are assigned for evaluating the individual's functional capacity in each of the six factors. Guidance for assigning numerical designators is contained in table 7-1. The numerical designator is not an automatic indicator of "deployability" or assignment restrictions, or referral to an MEB. The conditions listed in chapter 3 and the Soldier's functional limitations, rather than the numerical designator of the profile, will be the determining factors for MEB processing.

(1) An individual having a numerical designation of "1" under all factors is considered to possess a high level of medical fitness.

(2) A physical profile designator of "2" under any or all factors indicates that an individual possesses some medical condition or physical defect that may require some activity limitations.

(3) A profile containing one or more numerical designators of " 3 " signifies that the individual has one or more medical conditions or physical defects that may require significant limitations. The individual should receive assignments commensurate with his or her physical capability for military duty.

(4) A profile serial containing one or more numerical designators of " 4 " indicates that the individual has one or more medical conditions or physical defects of such severity that performance of military duty must be drastically limited.

$e$. Anatomical defects or pathological conditions will not of themselves form the sole basis for recommending assignment or duty limitations. While these conditions must be given consideration when accomplishing the profile, the prognosis and the possibility of further aggravation must also be considered. In this respect, profiling officers must consider the effect of their recommendations upon the Soldier's ability to perform duty. Profiles must be realistic. All profiles and assignment limitations must be specific, and written in lay terms. If the commander has questions about a profile or is unable to use the Soldier within the profile limitations, the procedures in paragraph 7-12 will apply.

(1) Determination of individual assignment or duties to be performed is a commander's decision. Limitations such 
as "no field duty," or "no overseas duty," are not proper medical recommendations. (However, they are included as administrative guidelines in pregnancy profiles.) Profiling officers will provide enough information regarding the Soldier's physical limitations to enable the nonmedical commander and AHRC to make a determination on individual assignments or duties. The profiling officer is responsible for entering the correct administrative code from table 7-2 into Item 2 of the DA Form 3349.

(2) It is the responsibility of the commander or personnel management officer to determine proper assignment and duty, based upon knowledge of the Soldier's profile, assignment limitations, and the duties of their grade and MOS.

(3) The commander has the final decision on the deployment of Soldiers in his/her unit. When medical providers and commanders disagree on the medical readiness status of a Soldier, the decision will be raised to the first general officer in the Soldier's chain of command, who will review both medical and commander recommendations and make the final decision whether to deploy the Soldier.

(4) Table 7-1 contains the physical profile functional capacity guide.

(5) See TB MED 287 for profiling Soldiers with pseudofolliculitis.

\section{7-4. Temporary vs. permanent profiles}

a. Electronic requirements. All temporary profiles greater than 30 days and all permanent profiles must be completed electronically. There are two ways to access the electronic profile; one through AHLTA (Version 3.3.2), and one through MODS.

(1) AHLTA. Providers first sign into AHLTA and then select the "Medical Readiness" link in AHLTA. From the Medical Readiness portal, the provider then selects the link for the e-Profile.

(2) $M O D S$. Providers can also access the e-Profile application at: https://apps.mods.army.mil.

Note. If the electronic systems are unavailable, the provider will issue a temporary profile in paper form for 30 days duration until the profile can be entered into e-Profile.

b. Permanent profiles. A profile is considered permanent unless a modifier of " $\mathrm{T}$ " (temporary) is added. A permanent profile may only be awarded or changed by the authority designated in paragraph 7-6, below. All permanent " 3 " and " 4 " profiles, for Soldiers on active duty, will be reviewed by an MEB physician or physician approval authority. An MEB physician is an MTF dedicated subject matter expert trained to perform disability evaluations per guidelines established in DODI 1332.38 (see AR 40-400 for MEB process). The MEB physician will assist the MTF commander in educating profiling officers on current physical profiling regulation and policy guidance.

(1) If the profile is permanent, the profiling officer must assess if the Soldier meets the medical retention standards of chapter 3. Those Soldiers on active duty who do not meet the medical retention standards must be referred to an MEB as per chapter 3. (See paras 9-10 and 10-25, respectively, for disposition of USAR and ARNG Soldiers not on active duty who do not meet medical retention standards.)

(2) Soldiers who have one or more condition(s) that do not meet medical retention standards are referred to a MEB / PEB after attaining the Medical Retention Determination Point (MRDP). The MRDP is when the Soldier's progress appears to have medically stabilized; the course of further recovery is relatively predictable; and where it can be reasonably determined that the Soldier is most likely not capable of performing the duties required of his MOS, grade, or rank. This MRDP and referral to a MEB/PEB will be made within 1 year of being diagnosed with a medical condition(s) that does not appear to meet medical retention standards, but the referral may be earlier if the medical provider determines that the Soldier will not be capable of returning to duty within 1 year. The MEB physician or physician approval authority will review all MEB referrals to insure that MRDP has been achieved prior to initiating a medical evaluation board; coordinate inappropriate MEB referrals back through the profiling officer for appropriate disposition; and assist physician approving authorities in reconciling profiling officer's questions and concerns about MRDP timing and MMRB versus MEB referrals. The MEB physician or physician approval authority will review all profiles to confirm that the MRDP has been reached before obtaining the approving authority signature.

(3) Those Soldiers (active duty and USAR/ARNG) who meet retention standards but have at least a 3 or 4 PULHES serial will be referred to a Medical MOS Retention Board (MMRB) in accordance with AR 600-60, unless waived by the MMRB convening authority.

(4) Permanent profiles may be amended (following the correct procedure) at any time if clinically indicated and will automatically be reviewed and verified by the privileged provider at the time of a Soldier's periodic health assessment or other medical examination.

(5) The Soldier's commander may also request a review of a permanent profile, in accordance with paragraph $7-12 b$.

c. Temporary profiles. Soldiers receiving medical or surgical care or recovering from illness, injury, or surgery, will be managed with temporary physical profiles until they reach the point in their evaluation, recovery, or rehabilitation where the profiling officer determines that MRDP has been achieved but no longer than 12 months. A temporary profile is given if the condition is considered temporary, the correction or treatment of the condition is medically advisable, and correction usually will result in a higher physical capacity. Soldiers on active duty and RC Soldiers not on active duty with a temporary profile will be medically evaluated at least once every 3 months at which time the profile may be extended for a maximum of 6 months from the initial profile start date by the profiling officer. 
(1) Temporary profiles exceeding 6 months duration, for the same medical condition, will be referred to a specialist (for that medical condition) for management and consideration for one of the following actions:

(a) Continuation of a temporary profile for a maximum of 12 months from the initial profile start date;

(b) Change the temporary profile to a permanent profile;

(c) Determination of whether the Soldier meets the medical retention standards of chapter 3 and, if not, referral to an MEB.

(2) The profiling officer must review previous profiles before making a decision to extend a temporary profile and refer the Soldier to a medical specialist for management if the temporary profile has been in effect for 6 months. Any extension of a temporary profile must be recorded on DA Form 3349, and if renewed, item 8 on the DA Form 3349 will contain the following statement: "This temporary profile is an extension of a temporary profile first issued on (date)."

(3) Temporary profiles will specify an expiration date. If no date is specified, the profile will automatically expire at the end of 30 days from issuance of the profile. In no case will Soldiers carry a temporary profile that has been extended for more than 12 months. If a profile is needed beyond the 12 months, the temporary profile will be changed to a permanent profile. Exceptions to the 12-month temporary physical profile restriction must be approved by the medical treatment facility (MTF) commander or their designated senior physician approval authority (often the deputy commander for clinical services).

\section{7-5. Representative profile serial and codes}

To facilitate the assignment of individuals after they have been given a physical profile serial and for statistical purposes, code designations have been adopted to represent certain combinations of physical limitations or assignment guidance (see table 7-2, below). The alphabetical coding system will be recorded on the DA Form 3349, item 2 and personnel qualifications records. Up to three different codes can be listed in item 2. This coding system will not be used on medical records to identify limitations. The numerical designations under each profile factor, PULHES, are given in table $7-1$, below.

\section{7-6. Profiling officer and approving authority}

a. Profiling officers. Commanders of Army MTFs are authorized to designate one or more physicians, dentists, optometrists, podiatrists, audiologists, nurse practitioners, nurse midwives, licensed clinical psychologists, and physician assistants as profiling officers. The commander will assure that those designated are thoroughly familiar with the contents of this regulation. Profiling officer limitations are as follows:

(1) Physicians. No limitations except for temporary profiles that exceed 6 months that require referral to a specialist (see para $7-4 c(1)$ ).

(2) Dentists, optometrists, physical therapists, chiropractors, and occupational therapists. No limitation within their specialty for awarding temporary or permanent numerical designators " 1 " and " 2 ." A temporary numerical designator "3" may be awarded for a period not to exceed 90 days. Any extension beyond 90 days must be signed by a physician. (See para 7-8.)

(3) Audiologists. No limitation within their specialty for awarding permanent numerical designators "1," "2," "3," or "4" in cases of sensorineural hearing loss, if retrocochlear lesion has been ruled out. Changing from or to a permanent numerical designator " 3 " or " 4 " requires the co-signature of a physician approving authority (see para 7-8).

(4) Physician assistants, nurse midwives, nurse practitioners, and licensed clinical psychologists. Limited to awarding temporary numerical designators "2," "3," and "4" for a period not to exceed 90 days. Any extension of a temporary profile beyond 90 days must be signed by a physician, except when the provisions of paragraph 7-9 apply. However, physician assistants with AOC 65DM1 certified in orthopedics have no limitations in awarding temporary orthopedic profiles or permanent profiles with a numerical designator of " 1 " or "2." Physician assistants, nurse midwives, nurse practitioners, and licensed clinical psychologists may award permanent profiles of "2", "3," or "4" provided the profile is signed by the physician approving authority.

(5) Podiatrists. No limitations within their specialty for awarding temporary or permanent profiles with a numerical designator of "1" or "2." Podiatrists may award permanent profiles of "3" or "4" providing the profile is signed by a physician approving authority.

(6) MEPS physicians, physician assistants, and nurse practitioners. They will also be designated as profiling officers. (See para 7-7b.)

(7) Other DOD physicians. In those instances where a Soldier does not have access to an Army MTF, but is assigned to a location with another Department of Defense medical facility (Navy, Air Force), a physician from another Service can be a profiling officer, if designated by the commander.

(8) AD TRICARE Prime Remote Soldiers, Selected Reserve (TPU, AGR, IMA) and ARNG Soldiers. These Soldiers may have profiles completed via the current agencies contracted to provide these medical services.

b. Approving authority. Commanders of Army MTFs are authorized to designate or delegate one or more physicians as approving authorities. The commander will assure that those designated are thoroughly familiar with the contents of 
this regulation. The approving authority must be a physician. Permanent " 3 " or " 4 " physical profiles require an approving authority signature.

\section{7-7. Recording and reporting of initial physical profile}

a. Individuals accepted for initial appointment, enlistment, or induction in peacetime normally will be given a numerical designator " 1 " or " 2 " physical profile in accordance with the instructions contained in this regulation. Initial physical profiles will be recorded on DD Form 2808 by the medical profiling officer at the time of the initial appointment, enlistment, or induction medical examination.

$b$. The initial physical profile serial will be entered on DD Form 2808 and also recorded on DD Forms 1966 (Record of Military Processing-Armed Forces of the United States), in the appropriate spaces. When the modifier "T" is entered on the profile serial, or in those exceptional cases where the numerical designator " 3 " is used on initial entry, a brief, nontechnical description of the defect will be recorded in the "Summary of Defects" section on the DD Form 2808, in addition to the exact diagnosis. All physical, geographic, or climatic area limitations applicable to the defect will also be entered in that section. If sufficient room for a full explanation is not available in that section, proper reference will be made in that section number and an additional sheet of paper attached. It is not uncommon for the MEPS to assign a profile with the numerical designator of " 3 " or " 0 " pending a medical waiver review of a disqualifying condition. This is for their administrative purposes only. If the individual receives a medical waiver, the waiver documentation completed by the waiver authority should indicate the appropriate profile in accordance with table $7-1$.

\section{7-8. Profiling reviews and approvals}

a. Permanent " 3 " or " 4 " profiles require the signatures of 2 profiling officers, one of which is a physician approving authority (unless the provisions of 7-8 $f$ apply). (Permanent profiles of " 3 " or " 4 " for the Individual Ready Reserve are valid with only one signature if signed by the AHRC Surgeon or his/her designee.) (ANG requires the signatures of 2 profiling officers for all permanent profiles to include permanent "1" or "2." See para 10-12). Temporary or permanent profiles of "1" or "2" require the signature of one profiling officer. See paragraph 7-6 to determine who is authorized to sign profiles.

$b$. Situations that require a mandatory review of an existing physical profile include-

(1) Return to duty of a Soldier hospitalized. The attending physician will ensure that the patient has the correct physical profile, assignment limitations(s), and medical followup instructions, as appropriate.

(2) When directed by the appointing authority in cases of a problematical or controversial nature requiring temporary revision of profile.

(3) At the time of the periodic health assessment or other medical examination.

(4) Upon request of the unit commander.

(5) On request of a PEB.

(6) When a permanent " 3 " or " 4 " profile is changed to a permanent " 1 " or " 2 " the change requires the signatures of 2 profiling officers, one of which is a physician approving authority (unless the provisions of 7-8f apply).

c. A temporary revision of profile will be completed when, in the opinion of the profiling officer, the functional capacity of the individual has changed to such an extent that it temporarily alters the individual's ability to perform duty. Temporary profiles written on DA Form 3349 will not exceed 3 months except as provided for in paragraphs 7-8d and 7-9. Temporary profiles written on DD Form 689 (Individual Sick Slip) will not exceed 30 days.

$d$. Tuberculous patients returned to a duty status who require anti-tuberculous chemotherapy following hospitalization will be given a temporary "2" profile under the P factor of the physical profile for a period of 1 year with recommendation that the Soldier be placed on duty at a fixed installation and will be provided the required medical supervision for a period of 1 year.

$e$. The physical profile in controversial or equivocal cases may be verified or revised by the hospital commander or command surgeon.

f. Physical profiles for Reserve Soldiers not on active duty and for those Soldiers activated on orders for greater than 30 days in the Ready Reserve (ARNG/AR), Standby Reserve (AR), and Retired Reserve (AR), may be accomplished by the U.S. Army Regional Support Command (RSC) surgeons, division staff surgeons, Active Army medical facility profiling officers (Reserve Soldiers on orders for 30 days or greater only), USAR/ARNG contracted agencies profiling officers, the U.S. Army Reserve (USARC) command surgeon and the AHRC command surgeon or their designees (Ready Reserve only). For ARNG/ARNGUS Soldiers not on active duty, profiles will be accomplished by State ARNG/ARNGUS providers. The respective State surgeons (if physician) or their designated physician alternate can be the approving authority for permanent "3" or "4" profiles. The NGB chief surgeon is also an ARNG approval authority for all ARNG Soldiers. The ARNG division surgeons may be designated as approval authority, but would require delegation by each concerned State or Territory State surgeon. Approval authorities for the Army Reserve are the USARC command surgeon and the Regional Support Command surgeons. The USAR operational and functional command surgeons and division surgeons that function as command surgeons may be delegated profile-approving authority by the USARC command surgeon. 
g. Individuals who were found unfit by a PEB but COAD used to be assigned a code "V" on their physical profile code. The code " $\mathrm{V}$ " is no longer used for this purpose but rather to identify Soldiers with restrictions on deployment. An " $X$ " is now used to identify individuals who were found unfit by a PEB but COAD or COAR.

$h$. MEB physicians must ensure that all physical profile and assignment limitations are fully recorded on one DA Form 3349. When the Soldier is referred to a PEB, a copy of the consolidated DA Form 3349 will be forwarded to the PEB with the MEB proceeding, with distribution of the form as indicated in paragraph 7-11b, below. On the consolidated DA Form 3349, the MEB physician may be the profiling officer (1st signature). Cooperation between the MEB physician, PEB liaison officers, and the PEB is essential when additional medical information or profile reconsideration is requested from the MTF by the PEB. The limitations described on the profile form may affect the decision of fitness by the PEB.

$i$. Table 7-1 will be used when determining the numerical designator of the PULHES factors. (For example, a Soldier will not be given a permanent " 3 " or " 4 " solely on the basis of a referral to a PEB.)

\section{7-9. Profiling pregnant Soldiers}

a. Intent. The intent of these provisions is to protect the fetus while ensuring productive use of the Soldier. Common sense, good judgement, and cooperation must prevail between policy, Soldier, and Soldier's commander to ensure a viable program. This profile has been revised from the previous profile published in the 1995 edition of this regulation. This profile guidance has been revised and includes mandating an occupational health interview to assess risks to the Soldier and fetus and adding additional restrictions to reduce exposure to solvents, lead, and fuels that may be associated with adverse pregnancy outcomes.

b. Responsibilities.

(1) Soldier. The Soldier will seek medical confirmation of pregnancy and will comply with the instructions of medical personnel and the individual's unit commander.

(2) Medical personnel. A privileged provider (physician, nurse midwife/practitioner or physician assistant) will confirm pregnancy and once confirmed will initiate prenatal care of the Soldier and issue a physical profile. Nurse midwives, nurse practitioners, and physician assistants are authorized to issue routine or standard pregnancy profiles for the duration of the pregnancy. An occupational history will be taken at the first visit to assess potential exposures related to the Soldier's specific MOS. This history is ideally taken by the occupational medicine physician or nurse. However, if this is not feasible, the profiling officer must complete the occupational history. After review of the occupational history, the profiling officer (physician, nurse midwife/practitioner, or physician assistant), in conjunction with the occupational health clinic as needed, will determine whether any additional occupational exposures, other than those indicated in the paragraphs below, should be avoided for the remainder of the pregnancy. Examples include but are not limited to hazardous chemicals, ionizing radiation, and excessive vibration. If the occupational history or industrial hygiene sampling data indicate significant exposure to physical, chemical, or biological hazards, then the profile will be revised to restrict exposure from these workplace hazards.

(3) Unit commander. The commander will counsel all female Soldiers as required by AR 600-8-24 or AR 635-200. The unit commander will consult with medical personnel as required. This includes establishing liaison with the occupational health clinic and requesting site visits by the occupational health personnel if necessary to assess any work place hazards.

c. Physical profiles.

(1) Profiles will be issued for the duration of the pregnancy. The MTF will ensure that the unit commander is provided a copy of the profile, and advise the unit commander as required. Upon termination of pregnancy, a new profile will be issued reflecting revised profile information. Physical profiles will be issued as follows:

(2) Under factor "P" of the physical profile, indicate "T-3."

(3) List diagnosis as "pregnancy, estimated delivery date."

d. Limitations. Unless superceded by an occupational health assessment, the standard pregnancy profile, DA Form 3349, will indicate the following limitations:

(1) Except under unusual circumstances, the Soldier should not be reassigned to overseas commands until pregnancy is terminated. (See AR 614-30 for waiver provisions and for criteria curtailing OCONUS tours.) She may be assigned within CONUS. Medical clearance must be obtained prior to any reassignment.

(2) The Soldier will not receive an assignment to duties where nausea, easy fatigue, or sudden lightheadedness would be hazardous to the Soldier, or others, to include all aviation duty, Classes 1/2/3. (However, there are specific provisions in para $4-13 c$ that allow the aircrew member to request and be granted permission to remain on flight status. ATC personnel may continue ATC duties with approval of the flight surgeon, obstetrician, and ATC supervisor.)

(3) Restrict exposures to military fuels. Pregnant Soldiers must be restricted from assignments involving frequent or routine exposures to fuel vapors or skin exposure to spilled fuel such as fuel handling or otherwise filling military vehicles with fuels such as mogas, JP8, and JP4.

(4) No weapons training in indoor firing ranges due to airborne lead concentrations and bore gas emissions. Firing of weapons is permitted at outdoor sites. (See (11) below, for other weapons training restrictions.) No exposure to 
organic solvent vapors above permissible levels. (For example, work in ARMS room is permitted if solvents are restricted to 1999 MIL-PRF-680, degreasing solvent.)

(5) No work in the motor pool involving painting, welding, soldering, grinding, and sanding on metal, parts washing, or other duties where the Soldier is routinely exposed to carbon monoxide, diesel exhaust, hazardous chemicals, paints, organic solvent vapors, or metal dusts and fumes (for example, motor vehicle mechanics). It does not apply to pregnant Soldiers who perform preventive maintenance checks and services (PMCS) on military vehicles using impermeable gloves and coveralls, nor does it apply to Soldiers who do work in areas adjacent to the motor pool bay (for example, administrative offices) if the work site is adequately ventilated and industrial hygiene sampling shows carbon monoxide, benzene, organic solvent vapors, metal dusts and fumes do not pose a hazard to pregnant Soldiers. (See (11), below, for PMCS restrictions at 20 weeks of pregnancy.)

(6) The Soldier must avoid excessive vibrations. Excessive vibrations occur in larger ground vehicles (greater than 1 $1 / 4$ ton) when the vehicle is driven on unpaved surfaces.

(7) Upon the diagnosis of pregnancy, the Soldier is exempt from regular unit physical fitness training and APFT testing/weight standards for the duration of the pregnancy and 180 days past pregnancy termination. After receiving medical clearance from their health care provider to participate in physical training, commanders will enroll Soldiers who are pregnant or postpartum to take part in the Army Pregnancy/Postpartum Physical Training (PPPT) program, an element of the Army Physical Fitness Training Program, in accordance with AR 350-1, Army Training and Education. The PPPT Program is designed to maintain health and fitness levels of pregnant Soldiers, and successfully integrate postpartum Soldiers back into unit physical fitness training programs with emphasis on achieving the APFT standards in accordance with guidance provided in the Army Physical Fitness Training Program, and meeting height/weight standards in accordance with guidance provided in the Army Weight Control Program. Pregnant and postpartum Soldiers must be cleared by their health care provider prior to participating in physical fitness training. Once pregnancy has been confirmed, the Soldier is exempt from wearing load bearing equipment (LBE) to include the web belt, individual body armor (IBA) and/or any other additional equipment. Wearing of individual body armor and/or any other additional equipment is not recommended and must be avoided after 14 weeks gestation.

(8) The Soldier is exempt from all immunizations except influenza and tetanus-diphtheria and from exposure to all fetotoxic chemicals noted on the occupational history form. The Soldier is exempt from exposure to chemical warfare and riot control agents (for example, nuclear, biological, and chemical training) and wearing MOPP gear at any time.

(9) The Soldier may work shifts.

(10) The Soldier must not climb or work on ladders or scaffolding.

(11) At 20 weeks of pregnancy, the Soldier is exempt from standing at parade rest or attention for longer than 15 minutes. The Soldier is exempt from participating in swimming qualifications, drown proofing, field duty, and weapons training. The Soldier must not ride in, perform PMCS on, or drive in vehicles larger than light medium tactical vehicles due to concerns regarding balance and possible hazards from falls.

(12) At 28 weeks of pregnancy, the Soldier must be provided a 15-minute rest period every 2 hours. Her workweek should not exceed 40 hours and the Soldier must not work more than 8 hours in any 1 day. The 8-hour work day does include one hour for physical training (PT) and the hours worked after reporting to work or work call formation, but does not include the PT hygiene time and travel time to and from PT.

$e$. Performance of duty. A woman who is experiencing a normal pregnancy may continue to perform military duty until delivery. Only those women experiencing unusual and complicated problems (for example, pregnancy-induced hypertension) will be excused from all duty, in which case they may be hospitalized or placed sick in quarters. Medical personnel will assist unit commanders in determining duties.

f. Sick in quarters. A pregnant Soldier will not be placed sick in quarters solely on the basis of her pregnancy unless there are complications present that would preclude any type of duty performance.

\section{7-10. Postpartum profiles}

a. Convalescent leave (as prescribed by AR 600-8-10) after delivery will be for a period determined by the attending physician. This will normally be for 42 days following normal pregnancy and delivery.

$b$. Convalescent leave after a termination of pregnancy (for example, miscarriage) will be determined on an individual basis by the attending physician.

c. Prior to commencing convalescent leave, postpartum Soldiers will be issued a postpartum profile. The temporary profile will be for 45 days. It begins on the day of child birth or termination of pregnancy and will allow PT at the Soldier's own pace. Soldiers are encouraged to use the AT-Home component of the ARMY PPPT Program while on convalescent leave. If a Soldier decides to return early from convalescent leave, the temporary profile remains in effect for the entire 45 days.

$d$. Soldiers will receive clearance from the profiling officer to return to full duty.

$e$. Postpartum (any pregnancy that lasts 20 weeks and beyond) Soldiers, in accordance with DODD 1308.1, are exempt from the APFT and from record weigh-in for 180 days following termination of pregnancy. After receiving clearance from their health care provider to resume physical fitness training, postpartum Soldiers will take part in the postpartum physical fitness training element of the Army. Postpartum Soldiers must receive clearance from their health 
care provider prior to returning to regular unit physical fitness training if it is before 180 days following pregnancy termination. After receiving clearance from their physician to resume physical training, they are expected to use the time in preparation for the APFT.

$f$. The above guidance will only be modified if, upon evaluation of a physician, it has been determined the postpartum Soldier requires a more restrictive or longer profile because of complicated or unusual medical problems.

\section{7-11. Preparation, approval, and disposition of DA Form 3349}

a. Preparation of DA Form 3349.

(1) The DA Form 3349 will be used to record both permanent profiles and temporary profiles. The DA Form 689 (Individual Sick Slip) may be used in lieu of DA Form 3349 for temporary profiles not to exceed 30 days and will include information on activities the Soldier can perform, as well as the physical limitations. An SF 600 will be used to attach additional information to the DA Form 3349 on the physical activities a Soldier can or cannot perform if there is inadequate space on the DA Form 3349. This additional SF 600 will be clearly labeled as a continuation of the DA Form 3349 .

(2) If electronic profiling is available, an electronic DA Form 3349 will be used for all profiles over 30 days duration.

(3) The DA Form 3349 will be prepared as follows:

(a) Item 1. Record medical conditions and/or physical defects in common usage, nontechnical language that a layman can understand. For example, "compound comminuted fracture, left tibia" might simply be described as "broken leg." The checkboxes labeled Injury and Illness/Disease are used for tracking purposes. Check the injury box if the Soldier's medical condition is the result of an injury; otherwise, check the box labeled Illness/Disease.

(b) Item 2. Code designations (defined in table 7-2) are limited to permanent profiles for administrative use only and are to be completed by the profiling officer. Up to three different codes can be listed. All functional and assignment limitations are recorded in item 8.

(c) Item 3. Enter under each permanent and temporary PULHES factors the appropriate profile serial code $(1,2,3$, and 4) as prescribed) for the specific PULHES factor. A Soldier may have a permanent profile for one condition and a temporary profile for another. All permanent profile blocks must be filled in. Only the applicable block under the temporary profile needs to be completed. For example, a Soldier with a sprained ankle who has permanent H3 hearing loss would be coded 111311 in the permanent PULHES space but _ $3_{-}$_under the temporary PULHES space.

(d) Item 4. Profile type. Check the appropriate block "a" or "b" for the type of profile. If the profile is temporary, enter the expiration date. If the profile is permanent, the profiling officer must assess if the Soldier meets retention standards of chapter 3 (Item 7).

(e) Item 5. Answer "Yes" or "No" to items 5a through 5j. These functional activities are the minimum requirements to be considered medically qualified for military duties worldwide and under field conditions. If any answer is "No" then the appropriate profile serial will in most cases be at least a 3 and the Soldier will be referred to a MEB. If the Soldier is able to do all the functional activities listed in 5 and meets the retention standards of chapter 3 , the Soldier will be referred to a MMRB in accordance with AR 600-60, unless waived by the MMRB convening authority.

(f) Item 6. Physical Fitness Test. Check either "Yes" or "No" to indicate whether the Soldier can perform the activities for the APFT. The "Yes" or "No" blocks on the alternate APFT need only be completed if the Soldier has restrictions for the regular APFT. If the Soldier cannot perform at least an alternate APFT the profile serial will be at least a 3 and referred to an MEB.

(g) Item 7. Those Soldiers (active duty and USAR/ARNG) who meet retention standards but have at least a permanent 3 or 4 PULHES (yes for item 7) serial will be referred to a MMRB in accordance with AR 600-60, unless waived by the MMRB convening authority. Those Soldiers on active duty who do not meet retention standards ("No" for item 7), must be referred to an MEB as per chapter 3. (See paras 9-10 and 10-26 for disposition of USAR and ARNG Soldiers not on active duty who do not meet medical retention standards.)

(h) Item 8. This space will be used to list any other physical activity restrictions or limitations not listed elsewhere on the form. In accordance with paragraph $7-4 b$, the profiling officer must review previous profiles before making a decision to extend a temporary profile. If this is an extension of a previous temporary profile, fill in the date of the original temporary profile in Item 8.

(i) Items 9, 10, and 11. Name and signature of profiling officer and date profile completed. Print name, grade and title of profiling officer, signature, and date. Permanent "1" or "2" profiles require the signature of one profiling officer. The signature of the profiling officer for " 1 " or " 2 " profiles is written in the section: "Typed name, grade, and title of profiling officer." Permanent "3" or "4" profiles require the signatures of two profiling officers, one of whom is the physician approving authority (unless the provisions of 7-8f apply). (See para 7-8 to determine who is qualified to be a profiling officer.) Temporary profiles require only the signature of one profiling officer except for extensions of profiles noted in paragraph 7-6a(2).

(j) Items 12, 13, and 14. Name and signature of approving authority and date reviewed. The approving authority will be designated by the MTF commander. (In the case of RC Soldiers not on active duty, see para 7-8f.) The approving 
authority for permanent " 3 " or "4" profiles must be a physician. If the approving authority does not concur with the profiling officer recommendation, the MTF commander will make the final decision.

(k) Item 15. How to access electronic profiles on Soldiers. Commanders can access the electronic profiles of Soldiers in their unit by going to http://www.mods.army.mil/ and clicking on "e-Profile" in MODS in the list of applications. Commanders are required to register and be approved to access the e-Profile module in MODS before they can gain access to the electronic profiles

(l) Item 16. Include patient identification: Name (Last, First); Grade/Rank; SSN (last 4 numbers or SSN); and the Soldier's unit.

(m) Item 17. Hospital or Medical facility.

(n) Item 18. Profiling Officer E-mail.

b. Disposition of DA Form 3349 (temporary or permanent) by the MTF. The electronic profile will be routed to the military personnel office (MILPO) and the Soldier's medical record. A paper copy of DA Form 3349 will be given to the Soldier. If the e-Profile is not available, a paper copy will be delivered by means other than the individual on whom the report is made to the following:

(1) Original to the Soldier's health record.

(2) One copy to the Soldier's commander.

(3) One copy to the MILPO.

c. Medical Protection System. The profiling officer (or approving authority if applicable) is responsible for ensuring the PULHES and Date of Profile is entered into the Medical Protection System (MEDPROS).

\section{7-12. Responsibility for personnel actions}

a. Commanders and personnel officers are responsible for necessary personnel actions, including appropriate entries on personnel management records and the assignment of the individual to military duties commensurate with the individual's physical profile and recorded assignment limitations.

$b$. If the Soldier's commander believes the Soldier cannot perform within the limits of the permanent profile, the commander will request reconsideration of the profile by the profiling physician. Reconsideration must be accomplished by the profiling officer, who will either amend the profile or revalidate the profile as appropriate. Commanders may also request a review of temporary profiles.

\section{7-13. Physical profile and the Army Weight Control Program}

The DA Form 3349 will not be used to excuse Soldiers from the provisions of AR 600-9. The AR 600-9 contains a standard memorandum for completion by a physician if there is an underlying or associated disease process that is the cause of the overweight condition. The inability to perform all APFT events or the use of certain medications is not generally considered sufficient medical rationale to exempt a Soldier from AR 600-9. 
Table 7-1

Physical profile functional capacity guide

\begin{tabular}{|c|c|c|c|c|c|c|}
\hline Profile & $P$ & $U$ & L & $\mathrm{H}$ & $E$ & $S$ \\
\hline Serial & Physical capacity & Upper extremities & Lower extremities & Hearing-ears & Vision-eyes & Psychiatric \\
\hline $\begin{array}{l}\text { Factors } \\
\text { to be } \\
\text { consid- } \\
\text { ered. }\end{array}$ & $\begin{array}{l}\text { Organic defects, } \\
\text { strength, stami- } \\
\text { na, agility, ener- } \\
\text { gy, muscular } \\
\text { coordination, } \\
\text { function, and } \\
\text { similar factors. }\end{array}$ & $\begin{array}{l}\text { Strength, range } \\
\text { of motion, and } \\
\text { general effi- } \\
\text { ciency of upper } \\
\text { arm, shoulder } \\
\text { girdle, and upper } \\
\text { back, including } \\
\text { cervical and tho- } \\
\text { racic vertebrae. }\end{array}$ & $\begin{array}{l}\text { Strength, range } \\
\text { of movement, } \\
\text { and efficiency of } \\
\text { feet, legs, lower } \\
\text { back and pelvic } \\
\text { girdle. }\end{array}$ & $\begin{array}{l}\text { Auditory sensitivity and } \\
\text { organic disease of the } \\
\text { ears }\end{array}$ & $\begin{array}{l}\text { Visual acuity, } \\
\text { and organic dis- } \\
\text { ease of the eyes } \\
\text { and lids. }\end{array}$ & $\begin{array}{l}\text { Type severity, and du- } \\
\text { ration of the psychiatric } \\
\text { symptoms or disorder } \\
\text { existing at the time the } \\
\text { profile is determined. } \\
\text { Amount of external } \\
\text { precipitating stress. } \\
\text { Predisposition as de- } \\
\text { termined by the basic } \\
\text { personality makeup, } \\
\text { intelligence, perform- } \\
\text { ance, and history of } \\
\text { past psychiatric disor- } \\
\text { der impairment of func- } \\
\text { tional capacity }\end{array}$ \\
\hline 1 & $\begin{array}{l}\text { Good muscular } \\
\text { development } \\
\text { with ability to } \\
\text { perform maxi- } \\
\text { mum effort for in- } \\
\text { definite periods. }\end{array}$ & $\begin{array}{l}\text { No loss of digits } \\
\text { or limitation of } \\
\text { motion; no de- } \\
\text { monstrable ab- } \\
\text { normality; able to } \\
\text { do hand to hand } \\
\text { fighting. }\end{array}$ & $\begin{array}{l}\text { No loss of digits } \\
\text { or limitation of } \\
\text { motion; no de- } \\
\text { monstrable ab- } \\
\text { normality; able to } \\
\text { perform long } \\
\text { marches, stand } \\
\text { over long peri- } \\
\text { ods, run. }\end{array}$ & $\begin{array}{l}\text { Audiometer average } \\
\text { level for each ear not } \\
\text { more than } 25 \mathrm{~dB} \text { at } 500 \text {, } \\
1000,2000 \mathrm{~Hz} \text { with no } \\
\text { individual level greater } \\
\text { then } 30 \mathrm{~dB} \text {. Not over } 45 \\
\mathrm{~dB} \text { at } 4000 \mathrm{~Hz} \text {. }\end{array}$ & $\begin{array}{l}\text { Uncorrected vis- } \\
\text { ual acuity } 20 / 200 \\
\text { correctable to } 20 / \\
20 \text {, in each eye. }\end{array}$ & $\begin{array}{l}\text { No psychiatric patholo- } \\
\text { gy. May have history } \\
\text { of a transient personal- } \\
\text { ity disorder. }\end{array}$ \\
\hline 2 & $\begin{array}{l}\text { Able to perform } \\
\text { maximum effort } \\
\text { over long peri- } \\
\text { ods. }\end{array}$ & $\begin{array}{l}\text { Slightly limited } \\
\text { mobility of joints, } \\
\text { muscular weak- } \\
\text { ness, or other } \\
\text { musculo-skeletal } \\
\text { defects that do } \\
\text { not prevent } \\
\text { hand-to-hand } \\
\text { fighting and do } \\
\text { not disqualify for } \\
\text { prolonged effort. }\end{array}$ & $\begin{array}{l}\text { Slightly limited } \\
\text { mobility of joints, } \\
\text { muscular weak- } \\
\text { ness, or other } \\
\text { musculo-skeletal } \\
\text { defects that do } \\
\text { not prevent mod- } \\
\text { erate marching, } \\
\text { climbing, timed } \\
\text { walking, or pro- } \\
\text { longed effort. }\end{array}$ & $\begin{array}{l}\text { Audiometer average } \\
\text { level for each ear at } \\
500,1000,2000 \mathrm{~Hz} \text {, } \\
\text { or } \\
\text { not more than } 30 \mathrm{~dB}, \\
\text { with no individual level } \\
\text { greater than } 35 \mathrm{~dB} \text { at } \\
\text { these frequencies, and } \\
\text { level not more than } 55 \\
\mathrm{~dB} \text { at } 4000 \mathrm{~Hz} \text {; } \\
\text { or } \\
\text { audiometer level } 30 \mathrm{~dB} \\
\text { at } 500 \mathrm{~Hz}, 25 \mathrm{~dB} \text { at } \\
1000 \text { and } 2000 \mathrm{~Hz} \text {, and } \\
35 \mathrm{~dB} \text { at } 4000 \mathrm{~Hz} \text { in } \\
\text { better ear. (Poorer ear } \\
\text { may be deaf.) }\end{array}$ & $\begin{array}{l}\text { Distant visual } \\
\text { acuity correcta- } \\
\text { ble to not worse } \\
\text { than } 20 / 40 \text { and } \\
20 / 70 \text {, or } 20 / 30 \\
\text { and } 20 / 100 \text {, or } \\
20 / 20 \text { and } 20 / \\
400 \text {. }\end{array}$ & $\begin{array}{l}\text { May have history of re- } \\
\text { covery from an acute } \\
\text { psychotic reaction due } \\
\text { to external or toxic } \\
\text { causes unrelated to al- } \\
\text { cohol or drug addic- } \\
\text { tion. }\end{array}$ \\
\hline 3 & $\begin{array}{l}\text { Unable to per- } \\
\text { form full effort } \\
\text { except for brief } \\
\text { or moderate peri- } \\
\text { ods. }\end{array}$ & $\begin{array}{l}\text { Defects or im- } \\
\text { pairments that } \\
\text { require signifi- } \\
\text { cant restriction of } \\
\text { use. }\end{array}$ & $\begin{array}{l}\text { Defects or im- } \\
\text { pairments that } \\
\text { require signifi- } \\
\text { cant restriction of } \\
\text { use. }\end{array}$ & $\begin{array}{l}\text { Speech reception } \\
\text { threshold in best ear not } \\
\text { greater than } 30 \mathrm{~dB} \mathrm{HL} \text {, } \\
\text { measured with or with- } \\
\text { out hearing aid; or } \\
\text { acute or chronic ear dis- } \\
\text { ease. }\end{array}$ & $\begin{array}{l}\text { Uncorrected dis- } \\
\text { tant visual acuity } \\
\text { of any degree } \\
\text { that is correcta- } \\
\text { ble not less than } \\
20 / 40 \text { in the bet- } \\
\text { ter eye. }\end{array}$ & $\begin{array}{l}\text { Satisfactory remission } \\
\text { from an acute psy- } \\
\text { chotic or neurotic epi- } \\
\text { sode that permits utili- } \\
\text { zation under specific } \\
\text { conditions (assignment } \\
\text { when outpatient psy- } \\
\text { chiatric treatment is } \\
\text { available or certain du- } \\
\text { ties can be avoided). }\end{array}$ \\
\hline 4 & $\begin{array}{l}\text { Functional level } \\
\text { below P3. }\end{array}$ & $\begin{array}{l}\text { Functional level } \\
\text { below U3. }\end{array}$ & $\begin{array}{l}\text { Functional level } \\
\text { below L3. }\end{array}$ & $\begin{array}{l}\text { Functional level below } \\
\text { H3. }\end{array}$ & $\begin{array}{l}\text { Visual acuity be- } \\
\text { low E3. }\end{array}$ & $\begin{array}{l}\text { Does not meet S3 } \\
\text { above. }\end{array}$ \\
\hline
\end{tabular}


Table 7-2

Profile codes*

\begin{tabular}{|c|c|c|}
\hline Code & Description/assignment limitation & Medical criteria (examples) \\
\hline CODE A & No assignment limitation. & $\begin{array}{l}\text { No demonstrable anatomical or physiological impair- } \\
\text { ment within standards established in table } 7-1 \text {. }\end{array}$ \\
\hline CODE B & $\begin{array}{l}\text { Soldier has minor impairments that may disqualify } \\
\text { for certain MOS training or assignment. }\end{array}$ & Minimal loss of joint motion, visual and hearing loss \\
\hline CODES D through $\mathrm{N}$ & $\begin{array}{l}\text { Possesses impairments that limit functions or assign- } \\
\text { ments. The codes listed below are for military per- } \\
\text { sonnel administrative purposes. Corresponding limi- } \\
\text { tations are general guidelines and are not to be } \\
\text { taken as verbatim limitations. (For example, a Sol- } \\
\text { dier with a code } D \text { may not be able to run but may } \\
\text { have no restrictions on marching or standing.) Item } 8 \\
\text { of DA Form } 3349 \text { will contain the specific limitations. }\end{array}$ & \\
\hline CODE D & No strenuous physical activity. & Organic cardiac disease, pulmonary insufficiency. \\
\hline CODE E & No continuous consumption of combat rations. & $\begin{array}{l}\text { Endocrine disorders-recent or repeated peptic ulcer } \\
\text { activity-chronic gastrointestinal disease requiring die- } \\
\text { tary management. }\end{array}$ \\
\hline CODE F & $\begin{array}{l}\text { No assignment or deployment to OCONUS areas } \\
\text { where definitive medical care for the Soldier's medi- } \\
\text { cal condition is not available. }\end{array}$ & $\begin{array}{l}\text { Individuals who require continued medical supervision } \\
\text { with hospitalization or frequent outpatient visits for se- } \\
\text { rious illness or injury. }\end{array}$ \\
\hline CODE H & $\begin{array}{l}\text { No duty where sudden loss of consciousness would } \\
\text { be dangerous to self or to others such as work on } \\
\text { scaffolding, vehicle driving, or near moving machin- } \\
\text { ery. }\end{array}$ & $\begin{array}{l}\text { Seizure disorders; other disorders producing syncopal } \\
\text { attacks of severe vertigo, such as Ménierè's syn- } \\
\text { drome. }\end{array}$ \\
\hline
\end{tabular}

CODE J

Given known handicaps associated with high frequency hearing loss similar to this, commanders are highly recommended to make an individual risk assessment of any Soldier with hearing loss that might be tasked to perform duties that require good hearing. For example, localization and detection of friend or foe sounds, scout, point, sentry, forward listening, post/observer, radio/telephone operator (RTO), and so forth. (See DA Pam 40-501, para 2-4, Combat readiness effects.) Hearing protection measures are required to prevent further hearing loss.

- 1-No exposure to noise in excess of $85 \mathrm{dBA}$ (decibels measured on the A scale) or weapon firing without use of properly fitted hearing protection. Annual hearing test required.

- 2- Further exposure to noise is hazardous to health. No duty or assignment to noise levels in excess of $85 \mathrm{dBA}$ or weapon firing (not to include firing for preparation of replacements for overseas movement (POR) qualification or annual weapons qualification with proper ear protection). Annual hearing test required.

- 3- No exposure to noise in excess of $85 \mathrm{dBA}$ or weapon firing without use of properly fitted hearing protection. This individual is "deaf" in one ear. Any permanent hearing loss in the good ear will cause a serious handicap. Annual hearing test required.

- 4-Further duty requiring exposure to high intensity noise is hazardous to health. No duty or assignment to noise levels in excess of $85 \mathrm{dBA}$ or weapon firing (not to include firing for overseas movement (POR) or weapon firing without use of properly ear protection). No duty requiring acute hearing. A hearing aid must be worn to meet medical fitness standards. 


\begin{tabular}{|c|c|c|}
\hline Code & Description/assignment limitation & Medical criteria (examples) \\
\hline CODE N & Limitations restricting wearing of combat boots. & $\begin{array}{l}\text { Any vascular or skin condition of the feet or legs that, } \\
\text { when aggravated by continuous wear of combat boots, } \\
\text { tends to develop unfitting ulcers. }\end{array}$ \\
\hline CODE S & $\begin{array}{l}\text { MEB. Soldier has been determined to meet medical } \\
\text { retention standards of Chapter } 3 \text { by a Medical Evalu- } \\
\text { ation Board (MEB). }\end{array}$ & \\
\hline CODE T & $\begin{array}{l}\text { Waiver granted for a disqualifying medical condition/ } \\
\text { standard for initial enlistment or appointment. The } \\
\text { disqualifying medical condition/standard for which a } \\
\text { waiver was granted will be documented in the Sol- } \\
\text { dier's accession medical examination. }\end{array}$ & \\
\hline CODE U & $\begin{array}{l}\text { Soldier has a limitation that needs to be considered } \\
\text { Individually as follows: (Briefly define limitation in } \\
\text { item } 8 \text {, comment section.) }\end{array}$ & $\begin{array}{l}\text { Any significant functional assignment limitation not } \\
\text { specifically identified elsewhere. }\end{array}$ \\
\hline CODE V & $\begin{array}{l}\text { Deployment. This code identifies a Soldier with re- } \\
\text { strictions on deployment to certain areas. }\end{array}$ & $\begin{array}{l}\text { Explanations of condition(s) and specific restrictions } \\
\text { are noted in the medical record. }\end{array}$ \\
\hline CODE W & $\begin{array}{l}\text { MMRB. This Soldier has a permanent } 3 \text { or } 4 \text { profile } \\
\text { who has been evaluated by a MMRB (MOS Medical } \\
\text { Review Board) with a recommendation to retain or } \\
\text { reclassify and returned to duty. }\end{array}$ & \\
\hline CODE X & $\begin{array}{l}\text { COAD/COAR. This Soldier is allowed to continue in } \\
\text { the military service with a disease, injury, or medical } \\
\text { defect that is below medical retention standards, pur- } \\
\text { suant to a waiver of retention standards under chap- } \\
\text { ter } 9 \text { or } 10 \text { of this publication, or waiver of unfit find- } \\
\text { ing and continued on active duty or in active Re- } \\
\text { serve status under AR } 635-40 \text {. }\end{array}$ & \\
\hline CODE Y & $\begin{array}{l}\text { Fit for duty. This Soldier has been determined to be } \\
\text { fit for duty (not entitled to separation or retirement } \\
\text { because of physical disability) after complete proces- } \\
\text { sing under AR 635-40. }\end{array}$ & \\
\hline
\end{tabular}

Notes:

"Codes do not automatically correspond to a specific numerical designator of the profile but are based on the general physical/assignment limitations.

\section{Chapter 8 \\ Medical Examinations-Administrative Procedures}

\section{8-1. General}

(See chap 6 for aviation administration procedures.) This chapter provides-

a. General administrative policies relative to military medical examinations.

$b$. Requirements for periodic medical examinations and periodic health assessments (PHA), separation, mobilization, and other medical examinations.

c. Policies relative to hospitalization of examinees for diagnostic purposes and use of documentary medical evidence, consultations, and the individual health record.

$d$. Policies relative to the scope and recording of medical examinations accomplished for stated purposes.

\section{8-2. Applications}

The provisions contained in this chapter apply to all medical examinations and PHAs accomplished at U.S. Army medical facilities or accomplished for the U.S. Army.

\section{8-3. Physical fitness}

a. Maintenance of physical and medical fitness is an individual military responsibility, particularly with reference to preventable conditions and remediable defects. Soldiers have an obligation to maintain themselves in a state of good physical condition so that they may perform their duties efficiently. Soldiers must seek timely medical advice whenever they have reason to believe that a medical condition or physical defect affects, or is likely to affect, their physical or mental wellbeing, or readiness status. They should not wait until the time of their annual periodic health assessment to make such a condition or defect known. Soldiers are responsible to seek medical care and report such medical care to 
their unit commanders. This reporting includes civilian health care. Civilian health records documenting a change which may impact their readiness status will be placed in the reserve component Soldier's military health record.

$b$. Commanders are responsible for ensuring the Soldier's readiness and medical status is properly documented in the personnel systems and the appropriate follow-up action is taken in regards to the Soldier's medical or readiness status.

c. The command the Soldier is assigned to is responsible for ensuring that Soldiers complete all medical readiness requirements.

\section{8-4. Consultations}

a. The use of specialty consultants, either military or civilian, is authorized in AR 40-400 and AR 601-270/AFR 33-7/MCO P-1100.75A.

$b$. A consultation will be completed in the case of an individual being considered for military service, including USMA and ROTC, whenever-

(1) Verification, or establishment, of the exact nature or degree of a given medical condition or physical defect is necessary for the determination of the examinee's medical acceptability or unacceptability based on prescribed medical fitness standards; or

(2) It will assist higher headquarters in the review and resolution of a questionable or borderline case; or

(3) The examining physician deems it necessary.

c. A consultation will be accomplished in the case of a Soldier on active duty whenever it is indicated to ensure the proper medical care and disposition of the Soldier.

$d$. A medical examiner requesting a consultation will routinely furnish the consultant with-

(1) The purpose or reason for which the individual is being examined; for example, enlistment.

(2) The reason for the consultation; for example, persistent tachycardia.

(3) A brief statement on what is desired of the consultant.

(4) Pertinent extracts from available medical records.

e. Reports of consultation will be appended to DD Form 2808 or PHA.

\section{8-5. Distribution of medical reports}

a. The original copy of the PHA will be filed as a permanent record in the heath record (AR 40-66), electronic health record (AHLTA), or outpatient treatment record. All IMR items will be documented on the DD Form 2766 and in MEDPROS. (When a bidirectional link is established between MEDPROS and AHLTA, information will be documented exclusively in the Soldier's electronic health record.) Copies may be reproduced from signed copies by any duplicating process that produces legible and permanent copies. Such copies are acceptable for any purpose unless specifically prohibited by the applicable regulation. Distribution of copies will not be made to unauthorized personnel or agencies.

$b$. DD Form 2808 and DD Form 2807-1-

(1) Are to be used for all remaining military examinations, to include, the cardiovascular screening program and examinations to attend special schools. Previous medical examinations/histories accomplished on Soldiers in accordance with this chapter should be considered valid. DD Form 2807-2 (Medical Prescreen of Medical History Report) is not required for military medical examinations.

(2) A minimum of two copies (both signed) of DD Form 2807-1 and DD Form 2808 will be prepared. One copy of each will be retained by the examining facility. The other copy will be giled as a permanent record in the heath record (AR 40-66) or outpatient treatment record. Special instructions for preparation and distribution of additional copies are contained elsewhere in this chapter or in other regulations dealing with programs involving or requiring medical examinations. Copies may be reproduced from signed copies by any duplicating process that produces legible and permanent copies. Such copies are acceptable for any purpose unless specifically prohibited by the applicable regulation. Distribution of copies will not be made to unauthorized personnel or agencies.

(3) In the case of general officers (grade O7 and above), the duplicate DD Form 2808 will be forwarded by the examining facility directly to Department of the Army, General Officer Management Office (DACS-GO), 200 Army Pentagon, Washington, DC 20310-0200.

c. In the case of general officers (grade $\mathrm{O} 7$ and above), the duplicate PHA form will be forwarded by the examining facility directly to Department of the Army, General Officer Management Office (DACS-GO), 200 Army Pentagon, Washington, DC 20310-0200.

\section{8-6. Documentary medical evidence}

a. Documentary medical records and other documents prepared by physicians or other individuals may be submitted by, or on behalf of, an examinee as evidence of the presence, absence, or treatment of a defect or disease, and will be given due consideration by the examiner(s). Submission and use of such documentary medical evidence is encouraged. If insufficient copies are received, copies will be reproduced to meet the needs of $b$ and $c$, below.

$b$. A copy of each piece of documentary medical evidence received will be appended to each copy of the DD Form 
2808 or PHA, and a statement to this effect will be made in the Summary of Defects section and cross-referenced by the pertinent item number or in the medical progress note.

c. When a report of consultation or special test is obtained for an examinee, a copy will be attached to each DD Form 2808 or PHA as an integral part of the medical report, and a statement to this effect will be made on the DD Form 2808 or PHA and cross-referenced by the pertinent item number or in the medical progress note.

\section{8-7. Facilities and examiners}

a. Physicians may perform medical examinations of any type except where a specific requirement exists for the examination to be conducted by a physician qualified in a specialty. Physician assistants, nurse practitioners, optometrists, audiologists, and podiatrists, properly qualified by appropriate training and experience, may accomplish such phases of the medical examination as are deemed appropriate by the examining physician. They may sign the report of medical examination for the portions of the examination they actually accomplish, but the supervising physician will sign the report of medical examination in all cases. Physician assistants and nurse practitioners, properly qualified by appropriate training and experience, may complete the PHA.

$b$. In general, medical examinations and PHAs conducted for the Army will be completed at facilities of the Armed Forces, using military medical officers on Active or Reserve duty, or full-time or part-time civilian employee physicians, with the assistance of dentists, physician assistants, nurse practitioners, optometrists, audiologists, and podiatrists. There may be contract agreements with civilian or Department of Veterans Affairs (DVA) facilities to perform military medical or PHAs or separation examinations for Active or Reserve Component Forces. In such cases, agreements must be worked out with the overseeing Army MTF or Reserve Command to ensure that the medical examinations and/or PHAs are reviewed by individuals who are familiar with the medical retention standards of chapter 3 (for example, military physicians) and can make a competent determination on whether the Soldier meets the medical retention standards of chapter 3 and is therefore medically fit for retention, retirement, or separation and accurately complete a physical profile (DA Form 3349), if necessary.

c. Medical examinations for qualification and admission to the USMA, the U.S. Naval Academy, the U.S. Air Force Academy, and the respective preparatory schools will be conducted in coordination with DODMERB. (See AR 40-29/ NAVMEDCOMINST 6120.2/AFR 160-13/CG COMDTINST M6120.8.)

\section{8-8. Hospitalization}

Whenever hospitalization is necessary for evaluation in connection with a medical examination or PHA, it may be provided as authorized in AR 40-400.

\section{8-9. Objectives of medical examinations}

The objectives of military medical examinations and periodic health assessments are to provide information-

$a$. To inform the individual of modifiable health risks and to identify potential lifestyle modifications.

$b$. To initiate treatment of illness.

c. To meet administrative and legal requirements.

$d$. To update information on current medical conditions, medications, review PULHES, and identify any readiness or deployment limiting conditions.

$e$. To update the IMR status of the Soldier.

\section{8-10. Recording of medical examinations}

$a$. The results of a medical examination will be recorded on DD Form 2808 and such other forms as may be required. (See AR 40-29/NAVMEDCOMINST 6120.2/AFR 160-13/CG COMDTINST M6120.8 for DODMERB forms.)

$b$. Results of the PHA will be recorded on the electronic PHA form. If the electronic version is not available, the PHA may be recorded on an SF 600.

c. PHA results will be electronically transferred to DD Form 2766 (Adult Preventive and Chronic Care Flowsheet) automatically.

$d$. As the electronic health record becomes available, it will be used to record medical examinations and the PHA. $e$. Results on the IMR status of the Soldier will be input into MEDPROS.

\section{8-11. Scope of medical examinations}

a. The scope of a medical examination is prescribed in paragraph 8-12 and will conform to the intended use of the examination.

$b$. Limited or screening examinations, special tests, or inspections required for specific purposes (for example, drivers, personnel exposed to industrial hazards, blood donors, food handlers) may be prescribed by other regulations.

$c$. Each abnormality, whether or not it affects the examinee's medical fitness to perform military duty, will be routinely described. All diagnoses and symptoms will be noted. 


\section{8-12. Medical examination requirements and required forms}

a. Required forms. The required form for all Army military medical examinations (not used for the PHA) is DD Form 2808. The "Laboratory Findings" section of this form may not contain enough space to include all required tests. If additional space is needed, the "Notes" section in box 73 may be used for that purpose. (MTFs are encouraged to use standard overprints, stamps, etc., in box 73 for that purpose.) Table 8-1 contains model entries and explanatory notes for every box on the DD Form 2808. All items are NOT required on all examinations.

b. All examinations. The following items ARE REQUIRED on ALL Army military medical examinations, not the PHA, and additional items may be accomplished if clinically indicated. See paragraphs (3) through (8) below for additional items required for special examinations. The box number from the DD Form 2808 that corresponds to the appropriate item to be completed is listed following each item.

(1) Administrative data. Date of examination (box 1), SSN (box 2), Name of examinee (box 3), Home address (current address, not "home of record" if different) (box 4), Home or contact telephone number (box 5), Grade/rank (box 6), Date of birth (box 7), Age (box 8), Sex (box 9), Race (box 10), Service (box 15a), Component (box 15b), Purpose of exam (box 15c), and Name of examining facility (box 16). (Name and SSN will also be completed on the top of pages 2 and 3 of the DD Form 2808.)

(2) Clinical evaluation section (boxes 17 through 39). This includes examination of head, face, neck, scalp, nose, sinuses, mouth, throat, ears (drums), eyes (includes ophthalmoscopic), heart, lungs, vascular system, anus, abdomen, upper and lower extremities, feet, spine, skin, breast exam, neurologic exam, and testicular exam on males. (Rectal exams are not required on all examinations. Pelvic exams and Pap tests are not required on all female examinations. See paras (3) through (8) below for specific requirements.)

(3) Dental section (box 43), usually completed by a physician or physician's assistant who will be noting any obvious gross abnormalities. This does not replace the dental examination by a dentist required in AR 40-3. The physician or physician assistant will check the box acceptable or unacceptable. The section in this item for dental "class" will not be completed unless it is completed by a dentist.

(4) Notes section (box 44) (to explain any abnormalities).

(5) Urinalysis for albumin and sugar (boxes $45 a$ and 45b).

(6) Miscellaneous measurements. Height (box 53), weight (box 54), temperature (box 56), pulse (box 57), blood pressure (box 58a), distant vision (box 61), near vision (box 63), and audiometer results (box 71a).

(7) Qualification for service (box 74a). For separation and retirement exams, qualification is based on whether the examinee meets the medical retention standards of chapter 3.

(8) Physical profile (box 74b). This section does not replace the requirements for a DA Form 3349 as described in chapter 7.

(9) Summary of defects (box 77).

(10) Recommendations (box 78).

(11) Name and signatures of examining physician assistants or nurse practitioner (boxes 81a and 81b), and of examining or approving physician (boxes $82 a$ and $82 b$ or $84 a$ and $84 b$ ).

c. Separation Health Assessments (See OTSG Policy memos for updates).

(1) The following four categories of Soldiers are to undergo a separation health assessment (SHA) prior to their separation from AD:

(a) A Soldier who is involuntarily separated from AD.

(b) A Soldier of a reserve component who is separated from AD to which he/she was called or ordered in support of a contingency operation, if the $\mathrm{AD}$ was for a period of 31 or more consecutive days (REFRAD).

(c) A Soldier who is separated from AD for which the member was involuntarily retained (Stop-Loss) under Section 12305 of Title 10 USCS in support of a contingency operation.

(d) A Soldier who is separated from AD who served pursuant to a voluntary agreement of the member to remain on $\mathrm{AD}$ in support of a contingency operation.

(2) The SHA will consist of-

(a) A current self-reported health status.

(b) A face-to-face interview with a physician, nurse practitioner, or physician assistant to review the Soldier's medical record to identify any complaints or potential AD service-related (incurred or aggravated) illness or injury.

(c) A hands-on physical examination if during interview process it is deemed necessary.

(d) A discussion of the "A" and "B" rated US Preventive Services Task Force recommendations based on the Soldier's age and gender (see table 8-4).

(e) An assessment regarding the Soldier's qualification for retention according to chapter 3 .

d. Examination for retirement or all other separations. (In accordance with para 8-24, retirement examinations are mandatory. Separation from the Active Army examinations are conducted on the request of the Soldier or if on review of the medical records it is clinically indicated.) In addition to the items listed in "All Examinations" ( $b(2)$ above), the following items are required:

(1) Prostate for males age 50 and older (box 30). 
(2) Rectal exam with stool for occult blood test for age 50 and older (box 30 for exam). (Use box 73 for occult blood results.)

(3) HCT or HGB (box 47)

(4) PSA test for males 50 and older. (Record results in box 52b.)

(5) Urine specific gravity and urine microscopic. (Record results in box 52c.)

(6) Chest x-ray (only for Soldiers 40 and older). (Record results in box 73.)

(7) Cholesterol. (Record results in box 73.)

(8) FBS for those 40 and older. (Record in box 73.)

(9) EKG for those 40 and over or if clinically indicated. (Record in box 73.)

(10) See paragraph $8-23 i$ for hepatitis screening requirements.

(11) DD Form 2697 (Report of Medical Assessment) will also be completed.

e. Initial examinations for appointment, enlistment, or induction. In addition to the items listed in "All Examinations" ( $b(2)$ above), the following items are required. (See AR 40-29/NAVMEDCOMINST 6120.2/AFR 160-13/CG COMDTINST M6120.8 for DODMERB exams.)

Note. MEPCOM will provide instructions to the MEPS on completion of the required forms for Army applicants. These instructions will include additional items on the DD Form 2808 that are to be used solely by the MEPS (for example, boxes 75, 79, and 80).

(1) Pregnancy testing on female applicants (box 46).

(2) HIV testing (box 49). (See AR 600-110.)

(3) Drug and alcohol test. (ROTC cadets will be tested during precommissioning physical (boxes 50 and 51).)

(4) Chest x-ray only if clinically indicated. (Record in box 73.)

(5) Pelvic exams and pap tests are not required, except in the case of an enlisted accession between the ages of 40 to 42 years old; then it is required.

(6) Color vision. (Record results in box 66.)

f. Initial exam for Special Forces, SERE, free fall parachute training (high altitude low opening (HALO), marine diving (Special Forces and Ranger combat diving) and other marine diving (MOS OOB). In addition to the items listed in "All Examinations" $(b(2)$, above), the following items are required:

(1) Rectal exam with stool for occult blood (required for Special Forces, SERE, HALO, Special Forces, and Ranger combat diving) (box 30 for exam). (Use box 73 for occult blood results.)

(2) HCT (box 47).

(3) HIV (box 49).

(4) Urine specific gravity and urine microscopic. (Record in box 52c.)

(5) Color vision (boxes 59 and 60).

(6) Refraction, if vision does not correct to $20 / 20$ in each eye with spectacle or contact lenses or if uncorrected vision is worse than 20/70 in either eye (not required for SERE) (box 62).

(7) Valsalva (required for diving and HALO only) (box 72b).

(8) Chest x-ray (frontal view only; not required for SERE). (Record in box 73.)

(9) EKG. (Record in box 73.)

(10) White blood cell count (diving and HALO only). (Record in box 73.)

(11) Sickle cell screen. (Record in box 73.)

(12) Glucose-6-phosphate dehydrogenase (MOS 00B diving, CDQC, and MFF only). (Record in box 73.)

(13) Total Cholesterol, LDL, HDL and Triglycerides (Record in box 73).

(14) The RPR (Record in box 73).

(15) Dental examination by a dentist (not required for SERE).

g. Additional examinations for female Soldiers on active duty or ADT tours in excess of 1 year (see paragraph $8-20 a)$.

h. Flying duty medical examinations. (See ATB 2, Army Flight Surgeon's Administrative Guide and chaps 4 and 6 of this regulation).

i. Airborne examinations. In addition to the items listed in "All examinations" $(b(2)$, above), the following items are required:

(1) Valsalva (box 72b).

(2) Color vision (boxes 59 and 60).

$j$. Examination for Ranger School. In addition to the items listed in "All Examinations" ( $b(2)$, above) the following items are required:

(1) Age 34 and under. Urinalysis with microscopy (box 52), HCT (box 47), HIV test within 2 years (box 49), Sickle Cell (box 73). An evaluation by a dentist is also required.

(2) Age 35 and older. Urinalysis with microscopy (box 52), HCT (box 47), HIV test within 2 years (box 49), FBS (box 73), CBC (box 52), Fasting Lipid Panel, EKG, Rectal exam with occult blood. An evaluation by a dentist is also 
required. The requirements in paragraph $8-25 d$ for indications of medical follow-up for elevated or abnormal test results should be followed for these exams on applicants 35 and older and the results forwarded with the medical examination to the Ranger School for review.

\section{8-13. Report of medical history forms}

a. Preparation of DD Form 2807-1. (DD Form 2807-2 (Medical Prescreen of Medical History Report) is not required.) This form is completed by the examinee prior to being examined. The DD Form 2807-1 must be prepared in all cases when the DD Form 2808 is also completed. It provides the examining physician with an indication of the need for special discussion with the examinee and the areas in which detailed examination, special tests, or consultation referral may be indicated. The information entered on this form is considered confidential and will not be released to unauthorized sources. The examinee should be informed of the confidential nature of his or her entries and comments. Trained enlisted medical service personnel and qualified civilians may be used to instruct and assist examinees in the preparation of the report, but will make no entries on the form other than the date of examination and the examining facility. The DD Form 2807-1 will normally be prepared in an original and one copy. All items will be completed. Responses will be typewritten or printed in ink.

$b$. Signature. The examinee will sign the form in black or dark blue ink.

c. The physician's (or physician assistant's or nurse practitioner's) summary and elaboration of the examinee's medical history.

(1) The physician (or physician assistant or nurse practitioner) will summarize and elaborate upon the examinee's medical history, and in the case of military personnel, the examinee's health record, cross-referencing their comments by item number. All items checked in the affirmative will be clarified and the examiner will fully describe all abnormalities including those of a non-disqualifying nature.

(2) If the examinee is applying for enlistment or appointment and answers reveal that he or she was previously rejected for military service or was discharged for medical reasons, the exact reason should be ascertained and recorded.

(3) A facsimile stamp will not be used for signature. The typed or printed name of the physician, physician assistant, or nurse practitioner and the date will be entered in the designated blocks. The physician, physician assistant, or nurse practitioner will sign in black or dark-blue ink.

\section{8-14. Validity times for DD Forms 2808}

a. Medical examinations will be valid for the purpose and within the periods prescribed below, provided there has been no significant change in the individual's medical condition. The DD Form 2808 will not be used for the PHA. The physician responsible for the final medical evaluation of the individual being examined will sign and date the report in Block 85. The date recorded in Block 85 on the DD Form 2808 will reflect the date the medical examination is completed.

(1) Medical examinations will be valid for 24 months from the date of medical examination to qualify for entrance into USMA, the USUHS, ROTC, OCS, USMA Preparatory School, induction, enlistment, initial appointment as a commissioned officer or warrant officer (with the exceptions noted in (2), below).

(2) At National Advanced Leaders Camp, a medical screening on DD Form 2807-1, with a focused medical examination if clinically indicated, and laboratory screening tests for DNA, HIV, and drug/alcohol testing will be accomplished. This medical screening and required laboratory tests will be used to qualify a cadet for continuation in ROTC and subsequent commission. The entry examination for USMA may be used as the commission examination providing the DNA, HIV and drug/alcohol tests have been accomplished during the cadet's tenure; and a DD Form 2807-1 is completed prior to commission with a focused medical examination performed if clinically indicated. The entry examination to qualify for Physician Assistant School may be used for the commission examination providing there has been no change in the student's medical condition since the last examination. (A DA Form 3081 will be completed.)

(3) See paragraph 6-8 for validity periods for FDMEs.

(4) When accomplished incident to retirement, discharge, or release from active duty, medical examinations or the separation health assessments are valid for a period of 12 months from the date of examination. If the examination or assessment is accomplished more than 6 months prior to discharge, or retirement (or 6 months prior to transition leave date if the Soldier requests it), DA Form 3081 (Periodic Medical Examination (Statement of Exemption)) will be attached to the original DD Form 2808 or assessment.

(5) See table 6-1 for FDMEs.

(6) Medical examinations are valid for 60 months from the date of medical examination to qualify for airborne training. If an ROTC or USMA Cadet examination was recorded on DD Form 2351 (DOD Medical Examination Review Board (DODMERB) Report of Medical Examination) instead of DD Form 2808, the examination is still valid. If the examination is older than 2 years, applicants for airborne school must complete DA Form 3081 and note if there has been any known change in their medical condition since the last examination. Any notes that there has been a change needs to be reviewed by a physician to ensure they meet airborne school medical standards. 
(7) Medical examinations are valid for 24 months from completion date of medical examination for entrance to all USAJFKSWCS schools. This includes SFAS; Special Forces Qualification Course (SFQC); MFF; Special Forces CDQC; and SERE training. (Military Freefall Jumpmaster, Dive Supervisor, and Diving Medical Technician (DMT) training are not initial qualification courses. As such, these courses only require a current MFF/CDQC physical that is valid for the period specified in $8-19 c$ (2.) Candidates for DMT, not on dive status, require an initial CDQC physical to attend this school.)

(8) A current (within the last 12 months) periodic health assessment or medical examination, to include all lab tests and consults, for Active Army Soldiers and ARNGUS and USAR Soldiers will be valid for reenlistment, attendance at Army or civilian schools, ADT, Active Duty Operational Support (ADOS), and temporary tour of active duty tours unless the specific school requires a medical examination or a shorter validity period (for example, special forces, diving school, or aviation training). (See para 8-20c for definition of a periodic medical examination for active and RC Soldiers. Shorter validity periods for Army Schools must be prescribed by Army regulation or DA pamphlet.) The periodic examinations or PHA will be valid only if there has been no change in the Soldier's medical condition since the last complete PHA or medical examination. USAR and ARNG/ARNGUS Soldiers will complete DA Form 3081 to indicate there has been no significant change since the last examination. See AR 600-110 for separate requirements for HIV testing.

(9) Medical examinations are valid for 18 months for entry into Ranger School, diving training (MOS OOB), and entry into aviation classes $1 / 2 / 3 / 4$.

$b$. Except for flying duty, discharge, or release from active duty, a medical examination or PHA conducted for one purpose is valid for any other purpose within the prescribed validity periods, provided the examination is of the proper scope specified in table $8-1$ or by the prescribing authority. If the examination is deficient in scope, only those tests and procedures needed to meet additional requirements need be accomplished and results recorded.

$c$. The periodic health assessment or medical examination obtained from members of the ARNG/ARNGUS and USAR as defined in paragraph $8-20 d(4)$ will be valid for the purpose of qualifying for immediate reenlistment in ARNG/ARNGUS and USAR, provided there has been no change in the individual's medical condition since their last complete medical examination. (See para 8-19 for requirements at mobilization or contingency operations.)

\section{8-15. Procurement medical examinations}

a. For administrative procedures pertaining to procurement medical examinations (para 2-1) conducted at MEPS, see AR 601-270/AFR 33-7/MCO P-1100.75A. For procedures pertaining to appointment and enlistment in the ARNG/ ARNGUS and USAR, see chapters 9 and 10 of this regulation. For procedures pertaining to enrollment in the Army ROTC, see AR 145-1. For procedures pertaining to USMA and ROTC Scholarship applicants, see AR 40-29/ NAVMEDCOMINST 6120.2/AFR 160-13/CG COMDTINST M6120.8.

$b$. The procurement medical examination will fulfill the requirement for a PHA for 1 year from the date of the examination (see also para 8-20).

\section{8-16. Active duty for training, active duty for special work, and inactive duty training}

$a$. Individuals on ADT/ADSW for 30 days or less are not required to undergo medical examinations prior to separation unless there is clinical indication for the examination.

$b$. An individual on ADT/ADSW will be given a medical examination if they incur an injury during such training that may result in disability or he or she alleges medical unfitness or disability.

c. Evaluation of medical fitness will be based on the medical fitness standards contained in chapter 3.

\section{8-17. Retiree Recalls}

A current (within the past 12 months) periodic health assessment, separation health assessment, or retirement medical examination is required.

\section{8-18. Health Records}

Medical examiners will review the health record (AR 40-66) of each examinee whenever an examination is conducted for the purpose of relief from active duty, resignation, retirement, separation from the Service, or when accomplished in connection with a periodic health assessment. The examiner will note on the DD Form 2766 (Adult Preventive and Chronic Care Flowseet) any significant problems, patient education, and follow-up care, as appropriate.

\section{8-19. Mobilization of units and members of Reserve Components of the Army}

A current periodic health assessment or a new medical examination is required incident to mobilization or call-up for war or contingency operations. See paragraph 8-24 for requirements for separation examinations.

\section{8-20. Periodic health assessments}

(See para $8-5$ for distribution of reports.)

a. Application.

(1) An annual periodic health assessment is required for all officers, warrant officers, and enlisted personnel of the 
Army, regardless of component. All general officers (brigadier general and above) on active duty will undergo a periodic health assessment every 2 years with a physical examination on the alternate years.

(2) Other than required medical surveillance and readiness monitoring, the periodic health assessment is not required for an individual who has undergone a medical examination within 1 year, the scope of which is equal to or greater than that of the required periodic health assessment (such as annual FDME/FDHS).

b. Procedure. The periodic health assessment consists of three parts-

(1) Part 1. A self-reported health status. A current self-reported health status and review, to include: A statement of health completed by the Soldier. The electronic version of the PHA, available on AKO is the preferable method of completing the PHA. Other acceptable forms to use for the statement of health, if the electronic version is not available, include the DD Form 2795 (Pre-Deployment Health Assessment Questionnaire), the DD Form 2796 (PostDeployment Health Assessment (PDHA)), DD Form 2900 (Post-Deployment Health Re-Assessment (PDHRA)), or the DD Form 2807-1 (Report of Medical History). The Health Assessment Review Tool (HART-R) will be used for the self-reported health status when it becomes available online. The PHA statement of health will include questions on tobacco use, stress, alcohol abuse, behavioral health and traumatic brain injury exposure as well as questions designed to meet The Joint Commission Accreditation. Whenever possible, the statement of health will be done prior to arrival at the clinic, medical facility, physical exam section, Soldier Readiness Site, or local detachment.

(2) Part 2. A review of the Soldier's height and weight, current medical conditions and deployment related health problems, to include screening for traumatic brain injury exposure, allergies, medications, required immunizations, update of medical readiness laboratory tests, audiology and optometry examination results. The DD Form 2766, Adult Preventive and Chronic Care Flow-sheet will be updated with the most current information. Medical readiness will be documented and/or updated electronically in MEDPROS during the encounter. Screening and patient education (as indicated by AHLTA reminders) will be done and documented to include stress, alcohol and tobacco use/abuse. The Soldier will be given written recommendations for age and gender appropriate screening laboratory and imaging procedures consistent with the U.S. Preventive Services Task Force (USPSTF) A and B recommendations (http://www. ahrq.gov/clinic/uspstfix.htm and table 8-4) and directed to discuss these recommendations with their Primary Care Manager (PCM).

(3) Parts 1 and 2 of the PHA may be conducted, coordinated and documented by any health care provider to include medics, nursing assistants, licensed practical nurses or registered nurses.

(4) Part 3. A physician, nurse practitioner or physician assistant will-

(a) Review the Soldier's statement of health, completed tests and reports, PULHES, and readiness screening information and make referrals as indicated.

(b) Perform a symptom focused exam to address concerns identified by the Soldier in their self reported health status. The exam will include an assessment for mental health disorders, behavioral health risks to include screening for traumatic brain injury exposure, and physical health conditions that may impact on mental status or emotional wellbeing. The exam will include an evaluation and treatment of medical problems and women's health services where indicated and authorized.

(c) Whenever possible, the PHA should be accomplished in a single appointment. Refer symptomatic Active Component Soldiers for evaluation and treatment. COMPO 1, TRICARE, Prime Remote, COMPO 2 and 3 Soldiers may complete the PHA via the current agencies contracted to provide these medical services.

(d) The physician, nurse practitioner or physician assistant will assess and document the Soldier's ability to be sustained in an austere environment for at least 30 days. An austere environment is defined as-

1. An area that regularly experiences significant environmental hazards (for example, heat, cold, altitude, aerosole particles) that would exacerbate existing medical conditions when protection (such as climate control) is not available.

2. An area that has limited access to a reliable source of electricity.

3. An area where force protection levels mandate prolonged use of body armor and or chemical protection equipment.

(e) Referrals will be submitted and orders entered for any required preventative or readiness related medical services not immediately available during the PHA process.

(f) The examining physician, nurse practitioner, or physician assistant will thoroughly investigate and document the Soldier's current medical status. The medical findings will be described in detail, using AHLTA if available, or an SF 507 (Clinical Record-Report on or Continuation of SF) or SF 600 (Chronological Record of Medical Care).

$(g)$ The PHA status will be reported as complete when the privileged provider reviews and confirms that the PHA has been completed and that all Unit Status Reportable (USR) IMR metrics (current health assessment, routine adult immunizations, HIV, temporary and permanent profiles, deployment-limiting condition(s) and pregnancy status) have been updated in the appropriate electronic systems.

(h) If the Soldier has recently been deployed, the date of completion of DD Form 2900, Post-Deployment Health Reassessment (PDHRA) will also be annotated in MEDPROS. An abbreviated PHA can be completed while completing Form 2796 and Form 2900. The abbreviated PHA is a separate assessment that is accessed while completing DD Form 2796 or DD Form 2900.

(5) Soldiers will be found qualified for retention if they meet the medical retention standards of chapter 3. 
(6) Soldiers who do not meet the medical retention standards of chapter 3 will be referred to an MEB. However, for RC and ARNG/ARNGUS Soldiers not on active duty, see chapters 9 and 10.

(7) All periodic health assessments will be reviewed by a physician, nurse practitioner or physician assistant.

(8) The Soldier will be counseled on remedial conditions found upon examination. Referrals will be made for the purpose of instituting care, continuing care for conditions already under treatment, and general health education matters including, but not limited to smoking, alcohol and drug abuse, and weight control.

(9) All personnel with potential hazardous exposures in their work environment for which medical surveillance examinations are required to ensure that there is no harmful effect to their health will receive appropriate medical surveillance examinations. Such examinations will be specific to job exposure.

c. Followup. Soldiers of the ARNG/ARNGUS or USAR who are not on active duty will be scheduled for follow-up appointment and consultations at Government expense when authorized. Treatment or correction of conditions or remediable defects as a result of examination will be scheduled if authorized. If individuals are not authorized treatment, they will be advised to consult a private physician of their own choice at their own expense.

d. Frequency. (See chap 6 for aviators, ATCs, and FSs.)

(1) All general officers (brigadier general and above) on active duty will undergo a periodic health assessment annually and a physical medical examination every 2 years.

(2) Special Forces/Ranger combat divers and MOS 00B divers must have a physical examination every 5 (five) years. The physical examination for divers must be performed by or reviewed by a DMO or a FS trained in diving medicine. The physical examination for MFF parachutists must be performed or reviewed by a FS every 5 years in conjunction with physiologic training.

(3) All other personnel on active duty will have a periodic health assessment on record no older than 12 months beginning after enlistment or commissioning. Military medical exams conducted for purposes other than the periodic health assessment may be used to comply with the periodic health assessment requirement.

(4) All members of the Selected Reserve not on active duty will have a periodic health assessment at least once every 12 months. Army commanders, the Commander, AHRC, and the Chief, NGB may, at their discretion, direct more frequent medical assessments or physical examinations in individual cases. MEDPROS data will reflect noncompliance if a PHA has not been reported or is older than 15 months.

(5) All members of the Ready Reserves not on active duty will have an annual periodic health assessment. The HART-R (when available) or DA Form 7349 (Initial Medical Review-Annual Medical Certificate) may be used for all Ready Reserve Soldiers to record the results of this periodic health assessment. A medical exam will be accomplished, if, upon review of the form, it is clinically indicated and authorized. The HART-R or DA Form 7349 will be filed in the individual's health record and DD Form 2766. DA Form 3725 (Army Reserve Status and Address Verification) (AR 135-133) is used to meet the annual periodic health assessment for all other Individual Ready Reserve Soldiers.

\section{8-21. Frequency of additional/alternate examinations}

a. Female examinations.

(1) In addition to the periodic health assessment, all women in the Army, regardless of age, on active duty or ADT/ ADSW tours in excess of 1 year or Active Guard-Reserve (AGR) tours will undergo pelvic examinations, to include a cervical cytologic screening test for cancer, annually. Breast examinations will be done in accordance with the USPSTF recommendations (see table 8-4). All women in the Army, under or equal to the age of 25, on active duty or ADT/ADSW tours in excess of 1 year or AGR tours will undergo annual testing for chlamydia. Periodic health assessments for ARNG/ARNGUS and USAR Soldiers not on active duty will include current (within 1 year) pelvic examinations and a cervical cytologic screening test for cancer. Civilian test results attached to the PHA for ARNG/ ARNGUS and USAR Soldiers not on active duty will be acceptable.

(2) All women in the Army on active duty (including AGR) or ADT tours in excess of 1 year will have a mammographic study accomplished in accordance with the USPSTF recommendations (see table 8-4). A record of the examination and test results will be maintained in the health record. More frequent mammographic studies may be performed if clinically indicated.

(3) Army applicants are not required to undergo a pelvic examination or a cytologic screening test unless they are between the ages of 40 to 42 years old. However, once enlisted or appointed, the provisions of paragraph $8-21 a(1)$ apply.

$b$. Medical surveillance examinations. The frequency of medical surveillance examinations varies according to job exposure. Annual or less frequent examinations will be performed during the birthday month. More frequent examinations will be scheduled during the birthday month and at appropriate intervals thereafter.

\section{8-22. Deferment of examinations}

a. Armywide or at specific installations. In circumstances requiring Armywide or installation deferment of periodic examinations (where conditions of the Service preclude the accomplishment of periodic examinations or periodic health 
assessments because resources are being directed to other missions (for example, screening for mobilization/contingency operations, heavy casualties, and so on), requests for exceptions to policies deferring examinations will be forwarded to TSG (ATTN: DASG-HS-AS).

b. Soldiers in isolated areas. Periodic health assessments may be delayed by the commander concerned for those Soldiers stationed in isolated areas; that is, Army attaches, military missions, and MAAGs, where medical facilities are not available. Periodic health assessments so delayed will be accomplished at the earliest opportunity in conjunction with leave, temporary duty, or when the individuals concerned are assigned or attached to a military installation having a medical facility. Medical examination of such individuals for retirement purposes may not be delayed.

c. Other deferments. In exceptional circumstances, in the case of an individual Soldier, where conditions of the service preclude the accomplishment of the annual periodic health assessment, it may be deferred by direction of the commander having custody of personnel files until such time as its accomplishment becomes feasible. An appropriate entry explaining the deferment will be made in the health record and on an SF 600 when such a situation exists.

\section{8-23. Promotion}

Officers, warrant officers, and enlisted personnel, regardless of component, are considered medically qualified for promotion on the basis of the annual periodic health assessment outlined in paragraph 8-20.

\section{8-24. Separation and retirement examinations}

a. AC and AGR Personnel (Title 10 or 32) Soldiers separating from the Army will be given a medical interview using DD Form 2697. The interview will be conducted by a physician, physician assistant, or nurse practitioner to document any complaints or potential service-related (incurred or aggravated) illness or injury. The Soldier must acknowledge with his or her signature in block 19 of the form that the information provided is true and complete. This form will be filed in the health record. See paragraph 8-24i for hepatitis screening requirements.

(1) Soldiers from AC, USARC and ARNG being considered for administrative separations, including but not limited to chapter 9, Alcohol or other Drug Abuse Rehabilitation Failure; chapter 13, Unsatisfactory Performance; chapter 513, Personality Disorder (PD); chapter 5-17, Other Mental Health Condition; and chapter 14-12, Patterns of Misconduct will be screened for both Post Traumatic Stress Disorder (PTSD) and mild Traumatic Brain Injury (mTBI) prior to their discharge. This screening must be in accordance with USAMEDCOM guidance and documented in the progress note located in the Soldier's AHLTA record. A Soldier will not be processed for administrative separation under AR 635-200, paragraph 5-17, if PTSD or mTBI are contributing factors to the diagnosis of PD, but will be evaluated under the physical disability system in accordance with AR 635-40.

(2) DODI 1332.14, and OTSG Policy Memo, prescribes the following requirements on separations based on Personality Disorder for enlisted Soldiers who have served or are currently serving in imminent danger pay areas-

(a) a psychiatrist or PhD-level psychologist must diagnose the PD;

(b) the diagnosis must be corroborated by MTF chief of behavioral health (or an equivalent official);

(c) the corroborated diagnosis, with all supporting medical documentation, will be forwarded for final review and endorsement by the Director, Proponency of Behavioral Health, Office of The Surgeon General (DASG-HSZ), 5109 Leesburg Pike, Suite 693, Falls Church, VA 22041-3258; and

(d) the diagnosis must address post traumatic stress disorder (PTSD) or other co-morbid mental illness, if present.

$b$. Soldiers separating from the Army will receive a separation medical examination if the Soldier requests it, or if, on review of the medical records or the DD Form 2697, a physician, a physician assistant, or a nurse practitioner feels an examination is appropriate (with exception noted in $c$, below). See table 8-2 for additional requirements based on the type of discharge. See $d$, below, for Soldiers retiring from active Service.

c. Separation Health Assessments (See OTSG Policy memos for updates).

(1) The following four categories of Soldiers are to undergo a separation health assessment (SHA) prior to their separation from AD:

(a) A Soldier who is involuntarily separated from AD. A Soldier who is involuntarily separated from AD. The Soldier must be available to complete the SHA. For incarcerated Soldiers in civilian prisons, the initial prison physical examination done by a credentialed provider meets the intent of an SHA.

(b) A Soldier of a reserve component who is separated from AD to which he/she was called or ordered in support of a contingency operation, if the $\mathrm{AD}$ was for a period of 31 or more consecutive days (REFRAD).

(c) A Soldier who is separated from AD for which the member was involuntarily retained (Stop-Loss) under Section 12305, Title 10 USC in support of a contingency operation.

(d) A Soldier who is separated from $\mathrm{AD}$ who served pursuant to a voluntary agreement of the member to remain on $\mathrm{AD}$ in support of a contingency operation.

(e) ARNG/ARNGUS or USAR Soldiers ordered to active duty for war, national emergency, or Presidential Select Reserve Call-Up (10 USC 12301(a), 12302, 12304, or 12305) will undergo a separation health assessment prior to mustering out of Federal service (ARNG/ARNGUS) or release from active duty (USAR). The scope of this screening (for example, medical interview with an individualized focused examination if clinically indicated vs. a complete medical examination) maybe changed by TSG prior to separation based on length of the mobilization/contingency 
operation and occupational exposures of the Soldiers. However, all Soldiers, as a minimum, will complete DD Form 2796 prior to mustering out of Federal service or release from active duty in accordance with a, above.

(2) The SHA is an individualized health assessment sufficient to evaluate the health of the Soldier at the time of discharge and will consist of-

(a) A current self-reported health status.

(b) A face-to-face interview with a physician, nurse practitioner, or physician assistant to review the Soldier's medical record to identify any complaints or potential $\mathrm{AD}$ service-related (incurred or aggravated) illness or injury.

(c) A hands-on physical examination if during interview process it is deemed necessary.

(d) A discussion of the "A" and "B" rated U.S. Preventive Services Task Force recommendations based on the Soldier's age and gender (see table 8-4).

(e) An assessment regarding the Soldier's qualification for retention according to chapter 3 .

(3) The SHA may only be waived if the Soldier has undergone a physical examination or assessment within 12 months prior to separation or discharge, and then only with the consent of the Soldier and concurrence of their unit commander.

d. Soldiers retiring from active duty to include AGR personnel (Title 10 or 32) are required to undergo a medical examination prior to retirement. The retirement medical examination may be a combined DOD/VA physical examination or a VA physical examination for the pilot programs, as long as the physician documents any complaints or potential service-related (incurred or aggravated) illness or injury and determines if the Soldier meets the medical retention standards of chapter 3.

$e$. Soldiers in paragraphs $a, b$, and $d$, above, who have indicated on DD Form 2697 that they intend to seek VA disability compensation or who have been referred to the Army Disability Evaluation System for determination of fitness will be given a standard VA compensation and pension physical, in conjunction with the combined DOD/DVA physical examination. For those Soldiers, the service medical record, proof of line of duty (LOD) determination, if necessary, and recent laboratory, radiological, and all other associated test results should accompany the claimant for VA benefits to the place of examination so that testing is not duplicated. A complete review of systems that documents the individual's physical condition at the time of retirement from the military service shall also be conducted as part of the physical examination to minimize duplication.

$f$. Voluntary requests for medical examinations should be submitted to the commander of the servicing MTF not earlier than 6 months nor later than 1 month prior to the anticipated date of separation or retirement (or if applicable and requested by the Soldier, 6 months prior to transition leave). If the examination is accomplished earlier than 6 months, $g$, below, applies.

$g$. When accomplished incident to retirement, discharge, or release from active duty, medical examinations, annual periodic health assessments, or separation health assessments are valid for a period of 12 months from the date of examination/assessment.

$h$. Soldiers who have been in medical surveillance programs because of hazardous job exposure will have a clinical evaluation and specific laboratory tests accomplished prior to separation even though a complete medical examination may not be required.

$i$. Soldiers requesting HCV screening will be tested. If the test is positive, medical evaluation to confirm HCV infection, to determine the need for specific treatments, and to provide counseling on lifestyle modifications and steps to protect others from infection will be accomplished. The following statements will overprinted on a DA Form 4700 (Medical Record-Supplemental Medical Data), administered and placed in the medical record for all Soldiers separating or retiring from active duty:

(1) Hepatitis $\mathrm{C}$ virus (HCV) is transmitted primarily by injections (for example, blood transfusions, contaminated needles, or sticks with contaminated sharp objects) of contaminated blood. The following are possible sources of HCV infection. If you can answer "yes" to any of these risk factors, you should receive a sample blood test to determine if you could have HCV. If you consider yourself at risk, based on an exposure to a possible source of HCV, you should have a simple blood test for HCV. You will not be asked to identify any specific risk factors to justify HCV testing. If the test is positive, you will receive a medical evaluation to confirm HCV infection, determine your need for specific treatments, and be provided counseling on lifestyle modifications and steps to protect others from infection.

(2) Risk factors are-

(a) Receiving a transfusion of blood or blood products before 1992.

(b) Ever injecting illegal drugs, including use once many years ago.

(c) Receiving clotting factor concentrates produced before 1987.

(d) Having chronic (long term) hemodialysis.

(e) Being told that you have persistent abnormal liver enzyme tests (alanine aminotransferase) or an unexplained liver disease.

(f) Receiving an organ transplant before July 1992.

(g) Having a needle stick, sharps, or mucosal exposure to potentially HCV-infected blood as part of your occupational duties and not having been previously evaluated for HCV infection. 
(3) If the test is positive, you will receive a medical evaluation to confirm HCV infection, determine your need for specific treatments, and be provided counseling on lifestyle modifications and steps to protect others from infection.

(4) Circle yes or no to the following responses and sign and date.

(a) No-I do not want to be tested for HCV.

(b) Yes-I want to be tested for HCV.

(c) Signature and date.

\section{8-25. Miscellaneous medical examinations}

a. The SFAS, SFQC, MFF parachutists, Special Forces/Ranger Combat divers, and SERE medical examination reports.

(1) Entrance into SFAS, SFQC, MFF parachuting, Special Forces/Ranger Combat diving, and SERE training will only be accomplished after meeting the medical fitness standards documented by the completion of the appropriate physical exam. The completed DD Form 2808 and DD Form 2807-1 (and supporting documents) must be reviewed and stamped "approved" by the U.S. Army Special Operations Command (USASOC) Surgeon's Office, or the surgeon's office that is designated by the USASOC Surgeon's Office as having review and approval authority.

(2) The Commander, USAJFKSWCS is the waiver authority for USAJFKSWCS schools. Individuals not meeting the medical fitness standards for USAJFKSWCS training courses will have their physicals and requests for waiver forwarded to Commander, USAJFKSWCS, ATTN: AOJK-GRP-C, Fort Bragg, NC, 28307-5217.

b. Certain geographic areas.

(1) When an individual is alerted for movement to or is placed on orders for assignment to the system of Army attaches, military missions, MAAGs, or to isolated areas, the commander of the station to which he or she is assigned will refer the individual and his or her dependents, if any, to the medical facility of the command.

(2) The physician of the facility will carefully review the health records and other available medical records of these individuals. Medical fitness standards and factors to consider in the evaluation are contained in paragraph 5-14. Review of the medical records will be supplemented by personal interviews with the individuals to obtain pertinent information concerning their state of health. The physician will consider such other factors as length of time since the last periodic health assessment or medical examination, age, and the physical adaptability of the individual to the new area.

(3) If, after review of records and discussion, it appears that a complete medical examination is indicated, a medical examination will be accomplished.

(4) The commander having processing responsibility will ensure that this medical action is completed prior to the individual's departure from his or her home station.

(5) If, as a result of their review of available medical records, discussion with the individual, and findings of the medical examination, the physician finds the individual medically qualified in every respect under paragraph 5-14c and qualified to meet the conditions that will be encountered in the area of contemplated assignment, he or she will complete and sign DA Form 3083 (Medical Examination for Certain Geographical Areas) prior to a permanent change of station. A copy of this statement will be filed in the health record or outpatient record (AR 40-66) and a copy forwarded to the commander who referred the individual to the medical facility.

(6) All family members will be screened following procedures in AR 608-75, Exceptional Family Member Program (EFMP). If any family member meets the criteria for enrollment in EFMP, the assignment will be coordinated with medical and educational representatives, as appropriate, to determine the availability of identified required services. If the medical needs cannot be met in the projected assignment area, the medical representative will recommend disapproval of accompanied family travel. The examiner will not disclose the cause of the disqualification of a dependent to the commander without the consent of the dependent, if an adult or a parent if the disqualification relates to a minor. If the Soldier or dependent is considered disqualified temporarily, the commander will be so informed and a reexamination scheduled following resolution of the condition.

(7) If the disqualification of the Soldier is permanent or if it is determined that the disqualifying condition will be present for an extended period of time, the physician may refer the Soldier to an MEB if the Soldier does not meet medical retention standards. The DA Form 3349 will be completed outlining specific limitations.

\section{8-26. Cardiovascular Screening Program (CVSP)}

a. The CVSP is required as part of the periodic health assessment for all the Active Army, ARNG/ARNGUS, and USAR (Selective Reserve) Soldiers age 40 and older. Cardiovascular screening will be done every five years unless otherwise clinically indicated. The CVSP begins with a Level 1 evaluation.

$b$. The Level I cardiovascular evaluation is conducted to determine the cardiovascular risk based on the presence of independent risk factors identified. The Level I evaluation will consist of:-

(1) Medical history to include family and smoking history.

(2) Medical records review.

(3) Blood pressure.

(4) Fasting lipid profile, including total cholesterol, LDL, HDL, and triglycerides. 
(5) Electrocardiogram (ECG).

(6) Fasting blood glucose.

(7) Height and weight.

(8) Calculation of a Framingham 10-year risk percentage. The National Cholesterol Education Program offers an online tool for calculating the Framingham 10-year coronary heart disease (CHD) risks. The site is: http://hin.nhlbi.nih. gov/atpiii/calculator.asp?usertype=prof and a downloadable version is at http://hin.nhlbi.nih.gov/atpii/riskcalc.htm. MEDPROS Framingham Tool (when available) may also be used.

$c$. Those Soldiers with the following findings on the ECG will require a Level II screening independent of the Framingham risk score:

(1) Any supraventricular tachycardia (3 or more beats at a rate greater than 100) to include atrial fibrillation/flutter, multifocal atrial tachycardia, junctional tachycardia, and persistent sinus tachycardia.

(2) Conduction disturbances of left bundle branch block (QRS duration more than $120 \mathrm{msec}$ ), Mobitz type II second degree atrioventricular (AV) block, and third degree AV block.

(3) Short PR interval (PR interval less than 120 milliseconds (msec) in all 12 leads).

(4) Sinus pause or asystole greater than 2 seconds in duration.

$d$. For those Soldiers with Framingham 10-year risk scores of between 10 percent and 15 percent, they will be referred to their primary care provider (PCM) for cardiac risk reduction treatment and education. The purpose of the medical referral is to confirm the presence of modifiable coronary risk factors and to advise and initiate medically appropriate treatments with the intent to modify cardiovascular risk. PCMs may further characterize risk by measuring C-Reactive Protein (CRP) and assessing for metabolic syndrome. If CRP is elevated or metabolic syndrome is present, the PCM should consider referring these Soldiers for Level II evaluation and reevaluating them annually.

(1) Primary care providers should use the National Cholesterol Education Program (NCEP) Adult Treatment Panel III (ATP III) or later version, the Seventh Report of the Joint National Committee on Prevention, Detection, Evaluation and Treatment of High Blood Pressure, and other appropriate clinical practice guidelines.

(2) If the Soldier is already under treatment and the Framingham 10-year risk score is less than 10 percent while on treatment, a separate referral for the purpose of the CVSP is not required. The medical records need to document the medical history, what treatment the Soldier is currently on, and where the Soldier is obtaining the treatment. If the risk score is between 10 percent and 15 percent, the Soldier will be referred back to his or her PCM for further care.

(3) Soldiers with hypertension should have a treatment plan for controlling his/her blood pressure documented by a PCM.

$e$. Except where otherwise eligible for military healthcare-

(1) Reserve Component Soldiers will be referred to their own medical provider for any further followup evaluation and treatment. The Soldier will provide copies of any records (pertaining to their medical management) from their civilian medical provider for inclusion in their military medical health record.

(2) If modification of risk factors improves the Framingham score sufficiently, the ARNG may clear the Soldier on the recalculated index and will not require any further screening or evaluation.

(3) If the modification of risk factors has not sufficiently improved the Soldier's Framingham score, or as required for clearance to participate in the APFT, the ARNG command surgeons or their clinical designees may authorize the specific cardiovascular screening tests as directed in AR 40-501, utilizing ARNG funds, to determine the CV status of the Soldier and/or deployment status.

$f$. For those Soldiers with a Framingham ten-year risk score of greater than 15 percent, a history of coronary heart disease (CHD) or a CHD-risk equivalent (peripheral arterial disease, abdominal aortic aneurysm, symptomatic carotid artery disease and diabetes), they must undergo a Level II evaluation. The Level II evaluation will consist of noninvasive testing using one (or more, when medically indicated) of the following tests:

(1) The Graded Exercise Stress Test (GXT; Bruce or modified Bruce protocols). If, during the GXT, blood pressure increases to greater than $200 \mathrm{mmHg}$ systolic or $100 \mathrm{mmHg}$ diastolic during or after exercise, an echocardiogram is recommended to rule out pathologic ventricular hypertrophy.

(2) Myocardial perfusion scintigraphy (thallium, sestamibi).

(3) Exercise and/or pharmacologic stress echocardiography.

(4) Once a Soldier has been cleared based on a Level II evaluation, reevaluation will be specified by the consulted cardiologist (or primary care doctor with the credentials to perform/interpret the Level II test). Typically, such Soldiers need reevaluation in 3 years; and sooner if they develop new symptoms or an increase in cardiac risk factors.

(5) Soldiers with metabolic syndrome (three or more of the following: waist greater than 40 inches for males and greater than 35 inches for females; triglycerides greater than $150 \mathrm{mcg} / \mathrm{dl}$; blood pressure greater than 130/85 mm Hg; HDL less than $40 \mathrm{mg} / \mathrm{dl}$; fasting blood glucose greater than or equal to $110 \mathrm{mg} / \mathrm{dl}$ ) are at increased risk for coronary artery disease and should be reevaluated at least annually. These Soldiers must be treated aggressively using a multimodal approach including pharmacologic therapy, weight reduction, diet counseling, exercise and control of other risk factors. 
(6) Soldiers who fail a Level II evaluation should receive a DA Form 3349 restricting them from strenuous activities until the Soldier is tested further and cleared for duty.

g. Medical records will be annotated that any required referrals have been made. All evaluations and recommendations from the medical followup examination on active and RC Soldiers will be placed in the medical record.

$h$. For all Soldiers upon reaching the age of 40, there is no need to require the cardiovascular screen prior to continuing PT and participating in the APFT. However, if a physician feels a profile restricting physical activity is warranted, they will complete the medical profile DA Form 3349 in accordance with chapter 7.

\section{8-27. Speech Recognition in Noise Test for H3 profile Soldiers}

$a$. The Speech Recognition in Noise Test (SPRINT) will be used by audiologists at all Army facilities to assess all H-3 Soldiers to provide recommendations concerning a potential communication handicap. The SPRINT will be administered by audiologists in a sound treated room, under earphones without use of hearing aides.

$b$. The tape-recorded test consists of monosyllabic words from the NU-6 lists in a background of speech babble noise. Normative data has been developed (see fig 8-1) so that the Soldier's score can be compared to a large sample of H-3 Soldiers' scores. This score, as a function of the Soldier's length in service, will be used to determine an appropriate recommendation based on table 8-3.

c. These recommendations should be made to MMRBs and MEBs, and considered when completing the physical profile assignment limitations on DA Form 3349. The recommendations provide appropriate information with which the boards can make a final determination.

\begin{tabular}{|c|c|c|}
\hline \multicolumn{3}{|c|}{$\begin{array}{l}\text { Table } 8-1 \\
\text { Recording of medical examination } 1\end{array}$} \\
\hline \multicolumn{2}{|c|}{ Item box number } & $\begin{array}{l}\text { Explanatory notes and Model entries (Model entries are in parentheses) } \\
\text { Refer to the glossary for acronyms and abbreviations used }\end{array}$ \\
\hline 1 & (Date of examination) & Enter the date on which the medical examination is accomplished. \\
\hline 2 & (Social security number) & Examinee's social security number. (SSN 396-38-0699) \\
\hline 3 & (Name) & $\begin{array}{l}\text { The entire last name, first name, and middle name are recorded. When Jr. or similar } \\
\text { designation is used, it will appear after the middle name. (Jackson, Charles John) }\end{array}$ \\
\hline 4 & (Home address) & $\begin{array}{l}\text { Examinee's current mailing address (not the "home of record"-if different) } \\
\text { (Street number, City, State, Zip Code or Unit mailing address). }\end{array}$ \\
\hline 5 & (Telephone number) & $\begin{array}{l}\text { Enter telephone number where the examinee can be reached-home or unit } \\
(202-555-1212) \text {. }\end{array}$ \\
\hline 6 & (Grade) & Enter examinee's grade $(\mathrm{E} 8, \mathrm{O} 4)$. \\
\hline 7 & (Date of birth) & Record as year, month, day. \\
\hline 8 & (Age) & List years of age at the time of examination ( $28 \mathrm{yr}$.) \\
\hline 9 & $(\operatorname{Sex})$ & Check female or male. \\
\hline 10 & (Race) & Check the applicable block. \\
\hline 11 & (Years of government service) & Not required. \\
\hline 12 & (Agency if not DOD) & To be used by other agencies, as appropriate. \\
\hline 13 & (Organization unit) & $\begin{array}{l}\text { The examinee's current military unit of assignment, Active or Reserve. If no current } \\
\text { military affiliation, enter a dash. } \\
\text { (for example, "B Company, 2D Battalion, 325th, Infantry, } 82 \text { nd Airborne Division, Fort } \\
\text { Bragg, NC 28307-5100") }\end{array}$ \\
\hline $14 a$ & $\begin{array}{l}\text { (Rating or specialty) } \\
\text { (Aviators only) }\end{array}$ & Not required on Army examinations unless directed by USAAMA. \\
\hline $14 b$ & $\begin{array}{l}\text { Total Flying Time } \\
\text { (Aviators only) }\end{array}$ & Not required on Army examinations unless directed by USAAMA. \\
\hline $14 \mathrm{c}$ & $\begin{array}{l}\text { Last } 6 \text { Months } \\
\text { (Flying Time - Aviators only) }\end{array}$ & Not required on Army examinations unless directed by USAAMA. \\
\hline $15 \mathrm{a}$ & (Service) & Check the appropriate service. \\
\hline $15 b$ & (Component) & Check the appropriate component. \\
\hline $15 c$ & (Purpose of examination) & Check or enter the purpose of the examination. \\
\hline
\end{tabular}




\begin{tabular}{|c|c|c|}
\hline \multicolumn{2}{|c|}{ Item box number } & \multirow{2}{*}{$\begin{array}{l}\text { Explanatory notes and Model entries (Model entries are in parentheses) } \\
\text { Refer to the glossary for acronyms and abbreviations used } \\
\text { Name of the examining facility or examiner and address. If an Army post office, in- } \\
\text { clude local national location (Military Entrance Processing Station, } 310 \text { Gaston Ave., } \\
\text { Fairmont, WV 12441-3217). }\end{array}$} \\
\hline 16 & (Name of examining facility) & \\
\hline $17^{2}$ & (Head, face, neck, scalp) & $\begin{array}{l}\text { Record all swollen glands, deformities, or imperfections of the head or face. If a defect } \\
\text { of the head or face, such as moderate or severe acne, cyst, exostosis, or scarring of } \\
\text { the face is detected, a statement will be made as to whether this defect will interfere } \\
\text { with the wearing of military clothing or equipment. If enlarged lymph nodes of the neck } \\
\text { are detected they will be described in detail and a clinical opinion of the etiology will } \\
\text { be recorded. }\end{array}$ \\
\hline 18 & (Nose) & $\begin{array}{l}\text { Record all abnormal findings. Record estimated percent of obstruction to airflow if } \\
\text { septal deviation, enlarged turbinates, or spurs are present. }\end{array}$ \\
\hline 19 & (Sinuses) & Record all abnormal findings ("Marked tenderness over left maxillary sinus"). \\
\hline 20 & (Mouth, throat) & $\begin{array}{l}\text { Record any abnormal findings. Enucleated tonsils are considered abnormal. } \\
\text { (Tonsils enucleated) }\end{array}$ \\
\hline 21 & (Ears) & $\begin{array}{l}\text { If operative scars are noted over the mastoid area, a notation of simple or radical } \\
\text { mastoidectomy will be entered (for example, "Bilateral severe swelling, injection and } \\
\text { tenderness of both ear canals"). }\end{array}$ \\
\hline 22 & (Eardrums) & $\begin{array}{l}\text { Record all abnormal findings. In the event of scarring of the tympanic membrane, the } \\
\text { percent of involvement of the membrane will be recorded as well as the mobility of the } \\
\text { membrane. If tested, a definite statement will be made as to whether the eardrums } \\
\text { move on valsalva maneuver or not and also noted in item } 72 \mathrm{~b} \text {. }\end{array}$ \\
\hline 23 & (Eyes) & $\begin{array}{l}\text { Record abnormal findings. If ptosis of lids is detected, a statement will be made as to } \\
\text { the cause and extent of the interference with vision. When pterygium is found, the fol- } \\
\text { lowing should be noted: } \\
\text { 1. Encroachment on the cornea, in millimeters, } \\
\text { 2. Progression, } \\
\text { 3. Vascularity. } \\
\text { For example, "Ptosis, bilateral, congenital. Does not interfere with vision. Pterygium, } \\
\text { left eye, } 3 \text { mm encroachment on cornea; nonprogressive, avascular." }\end{array}$ \\
\hline 24 & (Ophthalmoscope) & $\begin{array}{l}\text { Whenever opacities of the lens are detected, a statement is required regarding size, } \\
\text { progression since last examination, and interference with vision (for example, } \\
\text { "Redistribution of pigment, macular, Rt eye, no loss of visual function. No evidence of } \\
\text { active organic disease"). }\end{array}$ \\
\hline 25 & (Pupils) & Record all abnormal findings. \\
\hline 26 & (Ocular motility) & Record all abnormal findings. \\
\hline 27 & (Heart) & $\begin{array}{l}\text { Abnormal heart findings are to be described completely. Whenever a cardiac murmur } \\
\text { is heard, the time in the cardiac cycle, the intensity, the location, transmission, effect } \\
\text { of respiration, or change in the position, and a statement as to whether the murmur is } \\
\text { organic or functional will be included. When murmurs are described by grade, indicate } \\
\text { basis of grade (for example, "Grade II/IV soft, systolic murmur heard only in pulmonic } \\
\text { area and on recumbency, not transmitted. Disappears on exercise and deep inspira- } \\
\text { tions, physiological murmur"). }\end{array}$ \\
\hline 28 & (Lungs and chest) & $\begin{array}{l}\text { Lungs: If rales are detected, state cause. The examinee will be evaluated on the basis } \\
\text { of the cause of the pulmonary rales or other abnormal sounds and not simply on the } \\
\text { presence of such sounds (for example, "Sibilant and sonorous rales throughout chest. } \\
\text { Prolonged expiration"). } \\
\text { Breast exam: Note location, size, shape, consistency, discreteness, mobility, tender- } \\
\text { ness, erythema, dimpling over the mass, etc. }\end{array}$ \\
\hline 29 & (Vascular system) & $\begin{array}{l}\text { Adequately describe any abnormalities. When varicose veins are present, a statement } \\
\text { will include location, severity, and evidence of venous insufficiency (for example, } \\
\text { "Varicose veins, mild, posterior superficial veins of legs. No evidence of venous insuf- } \\
\text { ficiency"). }\end{array}$ \\
\hline 30 & $\begin{array}{l}\text { (Anus, rectum) } \\
\text { (Prostate if indicated) }\end{array}$ & $\begin{array}{l}\text { A definite statement will be made that exam has been performed. Note surgical scars } \\
\text { and hemorrhoids in regard to size, number, severity, and location. Check fistula, } \\
\text { cysts, and other abnormalities (for example, "One small external hemorrhoid, mild. } \\
\text { Digital rectal normal. Stool guaiac negative"). In prostate exam note grade of prostatic } \\
\text { enlargement, surface, consistency, shape, size, sensitivity, mobility. }\end{array}$ \\
\hline
\end{tabular}




\begin{tabular}{|c|c|c|}
\hline \multicolumn{3}{|c|}{$\begin{array}{l}\text { Table } 8-1 \\
\text { Recording of medical examination } 1 \text {-Continued }\end{array}$} \\
\hline \multicolumn{2}{|c|}{ Item box number } & \multirow{2}{*}{$\begin{array}{l}\text { Explanatory notes and Model entries (Model entries are in parentheses) } \\
\text { Refer to the glossary for acronyms and abbreviations used } \\
\text { Include hernia. Note any abdominal scars and describe the length in inches, location, } \\
\text { and direction. If a dilated inguinal ring is found, a statement will be included in item } 31 \\
\text { as to the presence or absence of a hernia ( } 2 \text {-inch linear diagonal scar, right lower } \\
\text { quadrant). }\end{array}$} \\
\hline 31 & (Abdomen, viscera) & \\
\hline 32 & (External genitalia) & Describe any abnormalities. Include results of testicular exam on males. \\
\hline 33 & (Upper extremities) & $\begin{array}{l}\text { Record any abnormality or limitation of motion. If applicant has a history of previous } \\
\text { injuries or fracture of the upper extremity, as, for example, a history of a broken arm } \\
\text { with no significant finding at the time of examination, indicate that no deformity exists } \\
\text { and function is normal. A positive statement is to be made even though the "normal" } \\
\text { column is checked. If a history of dislocation is obtained, a statement that function is } \\
\text { normal at this examination, if appropriate, is desired (for example, "No weakness, de- } \\
\text { formity, or limitation of motion, left arm"). }\end{array}$ \\
\hline 34 & (Lower extremities) & $\begin{array}{l}\text { Record any abnormality or limitation of motion. If applicant has a history of previous } \\
\text { injuries or fracture of the lower extremity, as, for example, a history of a broken leg } \\
\text { with no significant finding at the time of examination, indicate that no deformity exists } \\
\text { and function is normal. A positive statement is to be made even though the "normal" } \\
\text { column is checked. If a history of dislocation is obtained, a statement that function is } \\
\text { normal at this examination, if appropriate, is required (for example, "No weakness, de- } \\
\text { formity, or limitation of motion, left leg"). }\end{array}$ \\
\hline 35 & (Feet) & $\begin{array}{l}\text { Record any abnormality. When flat feet are detected a statement will be made as to } \\
\text { the stability of the foot, presence of symptoms, presence of eversion, stable, bulging } \\
\text { of the inner border, and rotation of the astragalus. Pes planus will not be expressed in } \\
\text { degree but should be recorded as mild, moderate, or severe (for example, "Flat feet, } \\
\text { moderate. Foot asymptomatic, no eversion or bulging; no rotation"). Circle category } \\
\text { relating to arch, degree, and symptoms. }\end{array}$ \\
\hline 36 & (Spine, other musculoskeletal) & $\begin{array}{l}\text { Include pelvis, sacroiliac, and lumbosacral joints. Check history. If scoliosis is de- } \\
\text { tected, the amount and location of deviation in inches from the midline will be stated. }\end{array}$ \\
\hline 37 & (Identifying body marks) & $\begin{array}{l}\text { Only scars or marks of purely identifying significance or those that interfere with func- } \\
\text { tion are recorded here. Tattoos that are obscene or so extensive as to be unsightly } \\
\text { will be described fully (for example, "1-in. vertical scar, dorsum; } 3 \text {-in. heart-left fore- } \\
\text { arm; shaped tattoo, lateral aspect middle } 1 / 3 \text { left arm"). }\end{array}$ \\
\hline 38 & (Skin) & $\begin{array}{l}\text { Describe pilonidal cyst or sinus. If skin disease is present, its chronicity and response } \\
\text { to treatment should be recorded. State also whether the skin disease will interfere with } \\
\text { the wearing of military clothing or equipment (for example, "Small discrete angular, flat } \\
\text { papules of flexor surface of forearms with scant scale; violaceous in color; umbilicated } \\
\text { appearance and tendency to linear grouping"). }\end{array}$ \\
\hline 39 & (Neurologic) & Record complete description of any abnormality. \\
\hline 40 & (Psychiatric) & $\begin{array}{l}\text { Record all abnormalities. Before a psychiatric diagnosis is made, a minimum psychiat- } \\
\text { ric evaluation will include Axis I, II, and III. }\end{array}$ \\
\hline 41 & (Pelvic) & $\begin{array}{l}\text { Note type of exam (for example, "bi-manual"). Record any abnormal findings. (See } \\
\text { item 52a for pap smear.) }\end{array}$ \\
\hline 42 & (Endocrine): & Describe every abnormality noted. \\
\hline 43 & (Dental) & $\begin{array}{l}\text { Examining physicians will apply the appropriate standards prescribed by chapters } 2 \text {, } \\
3,4 \text {, or } 6 \text {, and indicate "acceptable"or "non-acceptable." This does not replace the re- } \\
\text { quired annual dental examination by a dentist or the dentist's determination of the ap- } \\
\text { propriate dental classification. }\end{array}$ \\
\hline 44 & (Notes) & $\begin{array}{l}\text { Describe every abnormality noted. Enter pertinent item number before each comment. } \\
\text { Continue in item } 73 \text { if necessary. }\end{array}$ \\
\hline $45^{3}$ & $\begin{array}{l}\text { (Urinalysis) } \\
\text { a. Albumin } \\
\text { b. Sugar }\end{array}$ & Record results (For other urine microscopic or specific gravity, record in box 52c.) \\
\hline 46 & (Urine HcG) & Record results \\
\hline 47 & (Hemoglobin/hematocrit) & Record Results \\
\hline 48 & Blood Type & Record Results \\
\hline 49 & $(\mathrm{HIV})$ & Record date, results, add HIV specimen ID label in indicated section. \\
\hline 50 & (Drugs) & Record results of Drug Tests, add Drug Test Specimen ID to indicated space. \\
\hline
\end{tabular}




\begin{tabular}{|c|c|c|}
\hline \multicolumn{2}{|c|}{ Item box number } & \multirow{2}{*}{$\begin{array}{l}\text { Explanatory notes and Model entries (Model entries are in parentheses) } \\
\text { Refer to the glossary for acronyms and abbreviations used } \\
\text { Record results of alcohol screen }\end{array}$} \\
\hline 51 & (Alcohol) & \\
\hline 52 & (Other / results) & $\begin{array}{l}\text { 52a (use to record results of pap smear) } \\
52 \mathrm{~b} \text { (use to record PSA result) } \\
52 \mathrm{c} \text { (use to record urine microscopic or urine specific gravity.) }\end{array}$ \\
\hline 53 & (Height) & $\begin{array}{l}\text { Record in inches to the nearest quarter inch (without shoes). For initial Class } 1 \text { and in- } \\
\text { itial Class } 2 \text { (Aviator), and continuance Class } 2 \text { (Aviator) not previously measured: Leg } \\
\text { length, sitting height, and functional arm reach will be measured, in accordance with } \\
\text { Aeromedical policy letters. }\end{array}$ \\
\hline 54 & (Weight) & $\begin{array}{l}\text { Record in pounds to the nearest whole pound (in PT clothes without shoes, or hospi- } \\
\text { tal gown). }\end{array}$ \\
\hline 55 & (Maximal allowable weight) & $\begin{array}{l}\text { This item is for accession medical examinations only. This does not replace the official } \\
\text { weigh-in for Soldiers in conjunction with the APFT and AR 600-9 }\end{array}$ \\
\hline 56 & (Temperature) & Record in degrees Fahrenheit to the nearest tenth \\
\hline 57 & (Pulse) & Record with arm at heart level \\
\hline 58 a,b,c & (Blood pressure) & Record Results (for example, 110/76) $58 \mathrm{~b}$ and $\mathrm{c}$ are only required if elevated. \\
\hline 59 & (Red/green vision test) & $\begin{array}{l}\text { If examinee fails the color vision test in item } 66 \text {, he/she will be tested to ensure he/she } \\
\text { can distinguish between vivid red and vivid green and the results recorded as pass or } \\
\text { fail. }\end{array}$ \\
\hline 60 & (Other eye or vision test) & For example, results of red lens test. \\
\hline 61 & (Distant vision) & $\begin{array}{l}\text { Record in terms of the English Snellen Linear System }(20 / 20,20 / 30 \text {, etc.) of the un- } \\
\text { corrected vision of each eye. If uncorrected vision of either eye is less than } 20 / 20 \text {, en- } \\
\text { try will be made of the corrected vision of each eye (for example, "Right } 20 / 50 \text { cor- } \\
\text { rected (corr) to } 20 / 20 \text { and Left } 20 / 70 \text { corr to } 20 / 20 \text { "). }\end{array}$ \\
\hline 62 & (Refraction) & $\begin{array}{l}\text { The word "manifest" or "cycloplegic," whichever is acceptable, will be entered after re- } \\
\text { fraction. An emmetropic eye will be indicated by plano or } 0 \text {. For corrective lens, record } \\
\text { refractive value (for example, "Right By }-1.25 \mathrm{~S}-0.25 \mathrm{CX} 005 \text {. Left By }-1.75 \mathrm{~S}-0 \text {. } \\
25 \mathrm{CX} 175 \text { "). }\end{array}$ \\
\hline 63 & (Near vision) & $\begin{array}{l}\text { Record results in terms of reduced Snellen. Whenever the uncorrected vision is less } \\
\text { than normal }(20 / 20) \text {, enter the corrected vision for each eye and lens value after the } \\
\text { word "by" (for example, "Right } 20 / 40 \text { corr to } 20 / 20 \text { by Same and Left } 20 / 40 \text { corr to } 20 / \\
20 \text { by }+0.50 \text { "). }\end{array}$ \\
\hline 64 & (Heterophoria) & $\begin{array}{l}\text { Identify the test used; for example, either Maddox Rod or Stereoscope, Vision Testing } \\
\text { (SVT), and record results, Prism Divergence not required. All subjective tests will be } \\
\text { at } 20 \text { feet or at a distance setting of the SVT. Record distance interpupillary distance } \\
\text { (PD) in mm (for example, "Esophoria degree } 4 \text { Exophoria degree 0. right hyperphoria } \\
0 \text { left hyperphoria 0., PD 63"). }\end{array}$ \\
\hline 65 & (Accommodation) & $\begin{array}{l}\text { Record values without using the word "diopters" or symbols (for example, "Right 10.0; } \\
\text { Left } 9.5 \text { "). }\end{array}$ \\
\hline 66 & (Color vision) & $\begin{array}{l}\text { Record results in terms of test used, the results and the number of plates missed over } \\
\text { number of plates in test. The FALANT (USN) may be utilized. If the examinee fails ei- } \\
\text { ther of these tests, he or she will be tested for Red/Green vision and the results re- } \\
\text { corded in item } 59 \text { (for example, "PIP, pass, } 3 / 14 \text { or PIP, fail, } 9 / 14 \text { "). }\end{array}$ \\
\hline 67 & (Depth perception) & $\begin{array}{l}\text { Identify the test used. Record the results "Corrected" or "Uncorrected," as applicable. } \\
\text { Enter the score for Verhoeff or vision testing apparatus as "pass" or "fail" plus the } \\
\text { number missed over maximum score for that test (for example, "Verhoeff pass } 0 / 8 \text {; vi- } \\
\text { sion test apparatus (VTA) pass through D; VTA fail 1/9. Randot circles pass 0/10"). }\end{array}$ \\
\hline 68 & (Field of vision) & $\begin{array}{l}\text { Identify the test used and the results. If a vision field defect is found or suspected in } \\
\text { the confrontation test, a more exact perimetric test is made using a perimeter and/or } \\
\text { tangent screen. Findings are recorded on a visual chart and described in item } 77 \text {. } \\
\text { Copy of the visual chart must accompany the original DD Form } 2808 \text { (for example, } \\
\text { "Confrontation test: Normal, full"). }\end{array}$ \\
\hline 69 & (Night vision) & Test used and Score \\
\hline 70 & (Intraocular tension) & $\begin{array}{l}\text { Identify type of test used: applanation or non-contact. Record results numerically in } \\
\text { millimeters of mercury of intraocular pressure. Describe any abnormalities (for exam- } \\
\text { ple, "Normal O.D. } 18.9 \text { O.S. 17.3"). }\end{array}$ \\
\hline
\end{tabular}




\begin{tabular}{|c|c|c|}
\hline \multicolumn{2}{|c|}{ Item box number } & \multirow{2}{*}{$\begin{array}{l}\text { Explanatory notes and Model entries (Model entries are in parentheses) } \\
\text { Refer to the glossary for acronyms and abbreviations used } \\
\text { Test and record results at } 500,1000,2000,3000,4000 \text {, and } 6000 \text { Hertz using proce- } \\
\text { dures prescribed in DA Pam 40-501. ( } 71 \mathrm{~b} \text { is used for repeat tests if applicable) }\end{array}$} \\
\hline $71 a, b$ & (Audiometer) & \\
\hline $72 a$ & (Read Aloud Test) & Enter RAT satisfactory or unsatisfactory \\
\hline $72 b$ & (Valsalva) & Enter satisfactory or unsatisfactory \\
\hline 73 & (Notes) & $\begin{array}{l}\text { Examiner will enter notes on examination as necessary. Significant medical events in } \\
\text { the individual's life, such as major illnesses or injuries and any illness or injury since } \\
\text { the last in-service medical examination, will also be entered. Such information will be } \\
\text { developed by reviewing health record entries and questioning the examinee. Compli- } \\
\text { cations or sequelae, or absence thereof, will be noted where appropriate. Comments } \\
\text { from other items may also be continued in this space. } \\
\\
\text { This space is also used for additional tests when there is no specific box for the test } \\
\text { on the DD Form } 2808 \text {. For instance enter the results, if accomplished, of EKGs, chest } \\
\text { x-rays, FBS, Fasting lipid profile, cholesterol, occult blood tests, sickle cell screens. } \\
\text { Overprints or stamps may be used in this space. }\end{array}$ \\
\hline $74 a^{4}$ & (Examinee/applicant qualification) & $\begin{array}{l}\text { Indicate is qualified or not qualified for service. NOTE: EXAMINER SHOULD CORRE- } \\
\text { SPOND THIS WITH THE PURPOSE OF THE EXAMINATION AS CHECKED IN } \\
\text { ITEM 15c AND MUST CHECK EITHER QUALIFIED OR UNQUALIFIED IN THIS } \\
\text { SECTION AND INSERT WHAT THE Soldier/APPLICANT IS QUALIFIED FOR (FOR } \\
\text { EXAMPLE, "QUALIFIED FOR ACCESSION (Chap 2); QUALIFIED FOR RETEN- } \\
\text { TION (Chap 3); QUALIFIED FOR SEPARATION (Chap 3); QUALIFIED FOR RE- } \\
\text { TIREMENT (Chap 3)"). }\end{array}$ \\
\hline $74 b$ & (Physical profile) & $\begin{array}{l}\text { The physical profile as prescribed in chapter } 7 \text { will be recorded. Any permanent profile } \\
\text { with above a numerical designator of } 1 \text { should have a DA Form } 3349 \text { attached (for ex- } \\
\text { ample, "111121"). }\end{array}$ \\
\hline 75 & (Signature of examinee) & $\begin{array}{l}\text { The examinee will sign the DD Form } 2808 \text { if he/she has a disqualifying condition to in- } \\
\text { dicate that he/she has been advised of the disqualifying condition. }\end{array}$ \\
\hline 76 & (Significant or Disqualifying Defects) & $\begin{array}{l}\text { List the significant or disqualifying defects. On accession exams, list the correct ICD } 9 \\
\text { code from chapter } 2 \text { that corresponds to the disqualifying condition. Any medical waiv- } \\
\text { ers for accession should also be noted here. }\end{array}$ \\
\hline 77 & (Summary of defects) & $\begin{array}{l}\text { Summarize medical and dental defects considered to be significant. Those defects } \\
\text { considered serious enough to require disqualification or future consideration, such as } \\
\text { waiver or more complete survey, must be recorded. Also record any defect that may } \\
\text { be of future significance, such as nonstatic defects that may become worse. Enter } \\
\text { item number followed by a short, concise diagnosis; do not repeat the full description } \\
\text { of a defect that has already been described under the appropriate item. Do not sum- } \\
\text { marize minor, non-significant findings. }\end{array}$ \\
\hline 78 & (Recommendations) & $\begin{array}{l}\text { Notation will be made of any further specialized examinations or tests that are indi- } \\
\text { cated. }\end{array}$ \\
\hline 79 & (MEPS WORKLOAD) & (MEPS use only) \\
\hline 80 & $\begin{array}{l}\text { (Medical inspection date and physi- } \\
\text { cians signature) }\end{array}$ & $\begin{array}{l}\text { Used at the MEPS and includes inspection prior to movement to basic training of ht, } \\
\text { wt, body fat if applicable, pregnancy test and a note of qualified or unqualified. The } \\
\text { physician signature is the physician who has done the inspection and should not be } \\
\text { confused with items } 83-85 \text { that are the signatures of the medical examiners who ac- } \\
\text { complished and reviewed the medical examination. }\end{array}$ \\
\hline $81-84$ & (Physician or examiner) & $\begin{array}{l}\text { Enter the typed or printed names of examiner and signature (physician, physician as- } \\
\text { sistant (PA), or NP). If examination is not performed by a physician, a physician must } \\
\text { co-sign the form in item } 82 \text { a. }\end{array}$ \\
\hline 85 & Administrative review & $\begin{array}{l}\text { Any administrative review should be noted here by the signature of the reviewer, } \\
\text { grade and date. Also indicate the number of attached sheets if applicable. }\end{array}$ \\
\hline 86 & (Waiver Granted) & Indicate if a waiver was granted, date and by whom. \\
\hline 87 & (Number of attached Sheets) & List the number of any attached sheets needed. \\
\hline $\begin{array}{l}{ }^{1} \text { Not all items are required on all examinations. See paragraph 8-12 to determine the scope of the examination based on the purpose of the examination. } \\
2 \text { Note on the DD Form } 2808 \text {, items } 17 \text { though item 39, the examiner must check normal, abnormal or NE (not examined). All abnormalities will be described } \\
\text { in item } 44 \text { and continued in items } 73 \text { and } 77 \text { if needed. }\end{array}$ & $\begin{array}{l}\text { ms are required on all examinations. See pa } \\
\text { ne DD Form } 2808 \text {, items } 17 \text { though item } 39, t \\
\text { and continued in items } 73 \text { and } 77 \text { if needed. } \\
\text { two of the DD Form } 2808 \text {, re-enter the name } \\
\text { three of the DD Form } 2808 \text {, re-enter the nan }\end{array}$ & $\begin{array}{l}\text { agraph 8-12 to determine the scope of the examination based on the purpose of the examination. } \\
\text { e examiner must check normal, abnormal or NE (not examined). All abnormalities will be described } \\
\text { and social security number of the examinee in the spaces provided. } \\
\text { le and social security number of the examinee in the spaces provided. }\end{array}$ \\
\hline
\end{tabular}


Table 8-2

Schedule of separation medical examination or separation physical assessment ${ }^{\star}$

\begin{tabular}{|c|c|c|c|}
\hline Action & $\begin{array}{c}\text { Medical Examination } \\
\text { Required }\end{array}$ & $\begin{array}{l}\text { Separation Health } \\
\text { Assessment }\end{array}$ & $\begin{array}{l}\text { Not Required/Can be } \\
\text { requested by Soldier } \\
\text { (in writing) }\end{array}$ \\
\hline Retirement after 20 years or more of active duty & $\mathrm{X}$ & & \\
\hline $\begin{array}{l}\text { Retirement from active service for physical disability, permanent or tem- } \\
\text { porary, regardless of length of service. }\end{array}$ & $X$ & & \\
\hline $\begin{array}{l}\text { Expiration of term of active service (separation or discharge, less than } 20 \\
\text { years of service). }\end{array}$ & & & $X$ \\
\hline $\begin{array}{l}\text { Upon review of health record, evaluating physician or physician assistant } \\
\text { at servicing MTF determines that, because of medical care received dur- } \\
\text { ing active service, medical examination will serve the best interests of } \\
\text { Soldier and Government: for example, hospitalization for other than diag- } \\
\text { nostic purposes within } 1 \text { year of anticipated separation date. }\end{array}$ & $\mathrm{X}$ & & \\
\hline $\begin{array}{l}\text { Individual is member of the ARNGUS on active duty or ADT in excess of } \\
30 \text { days. }\end{array}$ & & $x$ & \\
\hline $\begin{array}{l}\text { Individual is member of the ARNGUS and has been called into Federal } \\
\text { service (see paragraph } 8-24 c \text { ). }\end{array}$ & & $\mathrm{X}$ & \\
\hline $\begin{array}{l}\text { Prisoners of war, including internees and repatriates, undergoing medical } \\
\text { care, convalescence or rehabilitation, who are being separated. }\end{array}$ & $\mathrm{X}$ & & \\
\hline $\begin{array}{l}\text { Officers, warrant officers, and enlisted Soldiers previously determined eli- } \\
\text { gible for separation or retirement for physical disability but continued on } \\
\text { active duty after complete physical disability processing (AR 635-40, } \\
\text { chapter } 6 \text {, and predecessor regulations). }\end{array}$ & $\begin{array}{l}\mathrm{X} \\
\text { (Plus MEB and } \\
\text { PEB) }\end{array}$ & & \\
\hline $\begin{array}{l}\text { Officers and warrant officers being processed for separation under provi- } \\
\text { sions of specific sections of AR } 600-8-24 \text { that specify medical examina- } \\
\text { tion and/or mental status evaluation. }\end{array}$ & $x$ & & \\
\hline $\begin{array}{l}\text { Officers and warrant officers being processed for separation under provi- } \\
\text { sions of specific sections of AR } 600-8-24 \text {, when medical examination } \\
\text { and/or mental status evaluation is not a requirement. }\end{array}$ & & & $\mathrm{X}$ \\
\hline $\begin{array}{l}\text { Enlisted Soldiers being processed for separation under provisions of AR } \\
635-200 \text {, chapter } 5 \text { : paras } 5-3 \text { (involuntary separations only), } 5-11 \text {, } \\
5-12 \text {, and } 5-17 \text { only, and chapters } 8,9,11 \text { (para } 11-3 b \text { only), } 12 \text {, and } \\
18 \text {. }\end{array}$ & & $\mathrm{X}$ & \\
\hline $\begin{array}{l}\text { Enlisted Soldiers being processed for separation under provisions of AR } \\
635-200 \text {, chapters } 13 \text { and } 14 \text { (sec III only), (both mental evaluation and } \\
\text { medical examination required). }\end{array}$ & $x$ & & \\
\hline $\begin{array}{l}\text { Enlisted Soldiers being processed for separation under provisions of AR } \\
635-200 \text {, chapter } 10 \text {. (If a medical examination is requested by the Sol- } \\
\text { dier, then mental status evaluation is required.) }\end{array}$ & & & $x$ \\
\hline Discharge in absentia (officers and enlisted Soldiers): & & & $\mathrm{x}$ \\
\hline Civil confinement. & & $\mathrm{X}$ & \\
\hline $\begin{array}{l}\text { When a Bad Conduct Discharge or a Dishonorable Discharge is upheld } \\
\text { by appellate review and the individual is on excess leave. }\end{array}$ & & $\mathrm{x}$ & \\
\hline Deserters who do not return to military control. & & & $\mathrm{X}$ \\
\hline $\begin{array}{l}\text { Enlisted Soldiers being processed for separation under all other provi- } \\
\text { sions of AR } 635-200 \text { not listed above. }\end{array}$ & & & $\mathrm{X}$ \\
\hline
\end{tabular}


Table 8-3

Results of Speech Recognition in Noise Test (SPRINT)

\begin{tabular}{l|l}
\hline Categories and Recommendations \\
\hline A & Retention in current assignment. \\
\hline B & Retention in current assignment with restrictions. \\
\hline $\mathrm{C}$ & Reassignment to, or retention in, non-noise hazardous area of concentration (AOC)/MOS. \\
\hline $\mathrm{D}$ & $\begin{array}{l}\text { Discretionary. (The audiologist should make a recommendation of Category C or E based on such factors as stability of } \\
\text { loss, potential for further noise exposure, the Soldier's AOC/MOS, and the recommendation of the Soldier's commander. } \\
\text { However, if the Soldier has } 18 \text { or more years of active Service, the audiologist may recommend Category B.) }\end{array}$ \\
\hline $\mathrm{E}$ & Separation from service. \\
\hline
\end{tabular}

Table 8-4

USPSTF Recommendations with Modifications in accordance with this regulation

\begin{tabular}{|c|c|c|}
\hline Test/Exam & Recommended start age or other criteria & $\begin{array}{l}\text { Requirements/Recommendations/Other } \\
\text { Clinical Considerations }\end{array}$ \\
\hline Mammography & 40 , then every $1-2$ years & $\begin{array}{l}\text { Performed with/without clinical breast exami- } \\
\text { nation. }\end{array}$ \\
\hline Cervical Cancer Screening & BCT/AIT & US Army requirement is for Annual Paps. \\
\hline Colorectal Cancer Screening & 50 & Screen all starting at age 50 . \\
\hline $\begin{array}{l}\text { High Blood Pressure Screening and HT/ } \\
\text { WT }\end{array}$ & BCT/AIT & Screen all at every medical encounter. \\
\hline Lipid Disorder Screening - Males & 35 & $\begin{array}{l}\text { Screen every } 5 \text { years and treat abnormal } \\
\text { lipids. }\end{array}$ \\
\hline Lipid Disorder Screening - Females & 40 & $\begin{array}{l}\text { Screen every } 5 \text { years and treat abnormal } \\
\text { lipids. }\end{array}$ \\
\hline CV disease risk & 40 & $\begin{array}{l}\text { Screen every } 5 \text { years or more often depend- } \\
\text { ing on risk, discuss ASA prophylaxis. }\end{array}$ \\
\hline $\begin{array}{l}\text { Osteoporosis Screening for } \\
\text { postmenopausal women }\end{array}$ & 60 & $\begin{array}{l}\text { For women at high risk for fractures, USPSTF } \\
\text { recommends that screening begin at age } 60\end{array}$ \\
\hline Chlamydia Screening & Sexually Active Females - Routinely & $\begin{array}{l}\text { Screening all active duty females younger } \\
\text { than } 25 \text {, and other asymptomatic women at in- } \\
\text { creased risk for infection. Cervical specimen is } \\
\text { not necessary for women not due for an an- } \\
\text { nual Pap smear. Urine test is acceptable. }\end{array}$ \\
\hline Gonorrhea Screening & Sexually Active Females-Routinely & $\begin{array}{l}\text { Screening all active duty females younger } \\
\text { than } 25 \text {, and other asymptomatic women at in- } \\
\text { creased risk for infection. Cervical specimen is } \\
\text { not necessary for women not due for an an- } \\
\text { nual Pap smear. Urine test is acceptable. }\end{array}$ \\
\hline Depression & 18 & $\begin{array}{l}\text { In clinical practices that have systems in place } \\
\text { to assure accurate diagnosis, effective treat- } \\
\text { ment, and follow-up. }\end{array}$ \\
\hline Alcohol Misuse Screening & 18 & All adults, including pregnant women. \\
\hline Tobacco Use & 18 & All adults, including pregnant women. \\
\hline
\end{tabular}




\section{CATEGORY}

\section{A}

B

$\mathrm{C}$

$\mathbf{D}$

$\mathbf{E}$

\section{RECOMMENDATION}

Retention in current assignment

Retention in current assignment with restrictions

Re-assignment to (or retention in) non-noise hazardous AOC/MOS

Discretionary **

Separation from service

** For soldiers falling in category D, the audiologist can make a recommendation associated with any category adjacent to Category $D$. Except for patients with $18+$ years on active duty (for which a Category $B$ recommendation could be made), this choice will be between Category $\mathrm{C}$ (re-assignment) or Category $\mathrm{E}$ (separation). The decision of which recommendation to make should be based on such factors as stability of loss, potential for further noise exposure, the soldier's AOC/MOS, and the recommendation of the local commander.

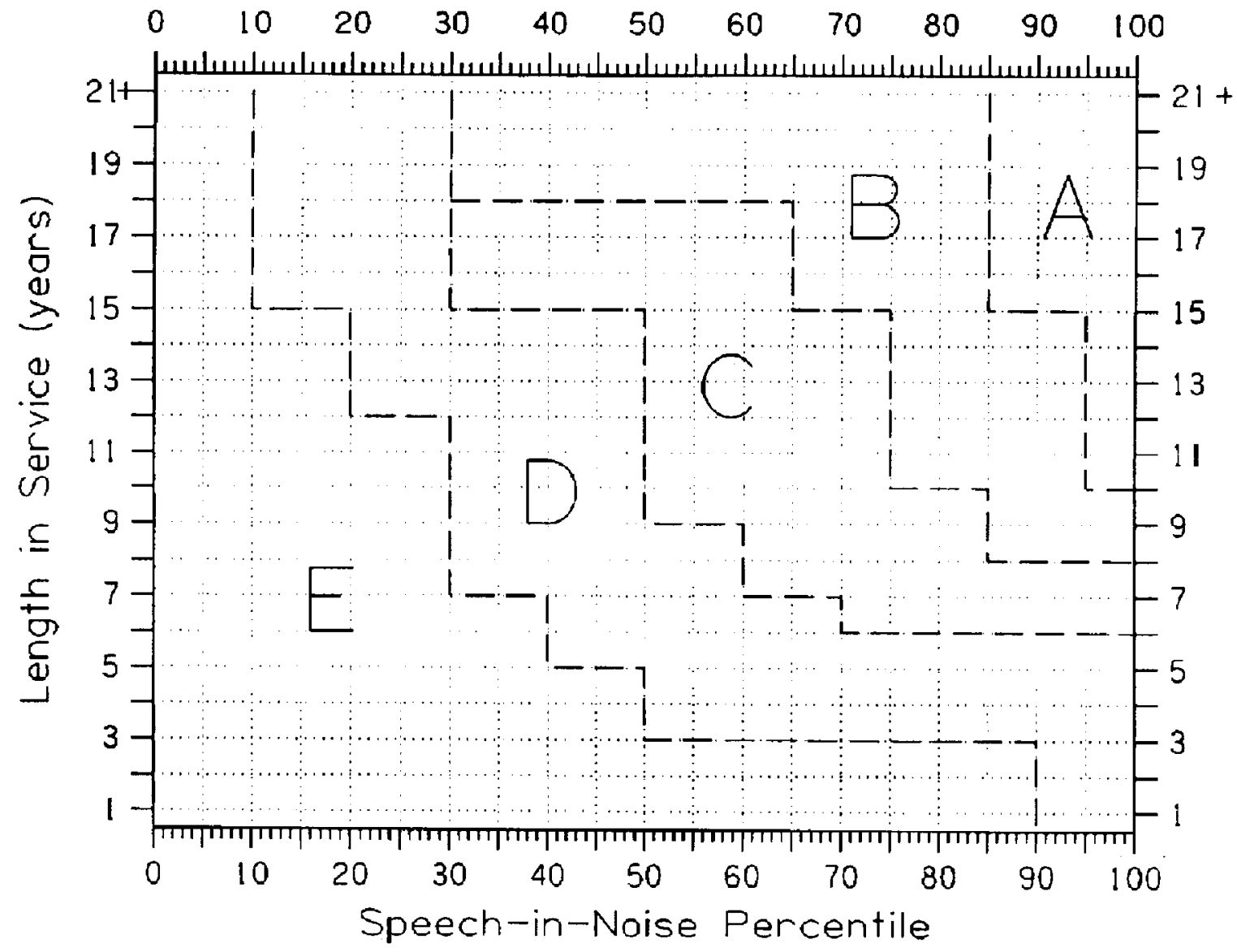

Figure 8-1. Normative data from speech recognition in noise test 


\section{Chapter 9 \\ Army Reserve Medical Examinations}

\section{9-1. General}

This chapter sets basic policies and procedures for medical examinations and the periodic health assessment.

\section{9-2. Application}

a. This chapter applies to the following personnel:

(1) Applicants seeking to enlist or be appointed as commissioned or warrant officers in the USAR. (Medical examinations for entrance into the Army ROTC program are governed by AR 145-1 and AR 145-2.)

(2) USAR members who want to be kept in an active Reserve status.

(3) USAR members who want to enter ADT and active duty.

$b$. This chapter does not apply to the Active Army or the ARNG/ARNGUS.

\section{9-3. Responsibility for medical fitness}

a. It is the responsibility of RC Soldiers to maintain their medical and dental fitness. This includes correcting remedial defects, avoiding harmful habits, and controlling weight. RC Soldiers are responsible for seeking medical advice and treatment quickly when they believe their physical wellbeing is in question. RC Soldiers must report to their unit commander, any change in their health status that impacts on their readiness status.

$b$. All RC Soldiers are responsible for providing the unit commander all medical documentation, including civilian health records, and completing the annual physical health assessment. Civilian health records documenting a change which may impact their readiness status will be placed in the Soldier's military health record.

\section{9-4. Examiners and examination facilities}

a. Applicants with prior service and RC Soldiers must present a letter of authorization to MEPS or Army medical facilities to receive a medical examination. (Applicants for initial enlistment who do not have prior military service will be examined only at MEPS.)

$b$. See paragraph $8-7 b$ for examination facilities.

\section{9-5. Examination reports}

For all examinations, the examiner will prepare and sign two copies each of DD Form 2808 and DD Form 2807-1. The examining facility will keep one set of these reports. The medical examiner will send the other set of these reports to the commander who authorized the examination. The authorizing commander will then handle these two reports as follows.

a. Reports prepared in examinations for appointment will accompany the application for appointment per AR 135-100.

b. Reports prepared in examinations of ready Reservists will be sent to the unit administrator. If the examination was not accomplished at a military medical facility or at the MEPS, the reports will then be sent to the review authorities named in paragraph 9-9. After review, they will be returned to the unit administrator to be filed in the Reservist's health record. (To ensure against loss, the unit administrator should keep a copy of the reports when sending them for review.)

\section{9-6. Conduct of examinations}

a. Conduct of examinations and the periodic health assessment.

$b$. See paragraph 8-14 for validity periods for medical examinations and the periodic health assessment.

\section{9-7. Types of examinations and their scheduling}

a. For annual physical health assessments and other examinations, including Special Forces, see chapter 8.

$b$. Ready Reservists released from active duty or ADT must take their first periodic examination in accordance with paragraph $8-20 d(5)$.

c. Commanders are responsible for taking proper corrective action whenever obligated Ready Reservists fail to accomplish their required annual health assessments and other examinations. Commanders are responsible to ensure the Soldier's readiness and medical status is properly documented in the personnel systems and the appropriate follow-up action is taken in regards to the Soldier's medical or readiness status.

\section{9-8. Physical profiling}

a. Examiners will determine and record physical profiles for Reservists per chapter 7. 
$b$. Profiling officers should be available within USAR medical units.

\section{9-9. Examination reviews}

Review of periodic examinations for RC Soldiers not on active duty is normally not required if the examination is accomplished at Army medical facilities or MEPS. Chief, USAR or his or her designee may initiate additional reviews if appropriate. (See chap 6 for aviation reviews and chap 1 for all other reviews and waiver authorities.)

\section{9-10. Disposition of medically unfit Reservists}

$a$. Normally, Reservists who do not meet the fitness standards set by chapter 3 will be transferred to the Retired Reserve per AR 140-10 or discharged from the USAR per AR 135-175 or AR 135-178. They will be transferred to the Retired Reserve only if eligible and if they apply for it.

$b$. Reservists who do not meet medical retention standards may request continuance in active USAR status in accordance with paragraph 9-11 below. In such cases, a medical impairment incurred in either military or civilian status will be acceptable; it need not have been incurred only in the LOD. Reservists with nonduty related medical conditions who are pending separation for not meeting the medical retention standards of chapter 3 may request referral to a PEB for a determination of fitness in accordance with paragraph 9-12 below.

\section{9-11. Requests for continuation in the USAR}

a. Requests for continuance will include-

(1) A copy of the most recent periodic medical examination or PHA.

(2) Any additional medical examinations, consultations, and hospitalization or treatment records pertaining to the unfitting condition. Civilian records are acceptable.

(3) A summary of the Reservist's experience and qualifications.

(4) An evaluation by the Reservist's unit commander of the Soldier's potential value to the military Service and the ability of the Soldier to perform the duties of his or her primary MOS and grade.

$b$. Requests for continuance will be sent to the Commander, AHRC, who will consider each request and determine if the Reservist's experience and qualifications are needed in the Service.

c. Each request for continuance will also be reviewed by the Surgeon, AHRC; he or she will determine if-

(1) The disability may adversely affect the Reservist's performance of active duty. The Reservist's grade, experience, and qualifications must be considered when determining this.

(2) The rigors of active service would aggravate the condition so that further hospitalization, time lost from duty, or a claim against the Government might result.

$d$. Waivers requested for officers being considered for assignment/selection to and within the general officer grades will be sent to the Chief, USAR for review and final determination. The Chief, USAR will consider each request and determine if the Reservist's experience and qualifications are needed in the Service. Each request will be reviewed by TSG, who will determine whether-

(1) The disability may adversely affect the Reservist's performance of active duty as a general officer (07 and above).

(2) The rigors of active service would aggravate the condition so that further hospitalization, time lost from duty, or a claim against the Government might result. The Chief, USAR must consider TSG's review when making a final determination.

(3) Cases where the opinions of TSG and Chief, USAR differ concerning officer(s) being considered for assignment/ promotion to and within general officer ranks will be forwarded to ODCS, G-1, ATTN: DAPE-GO, 300 Army Pentagon, Washington, DC 20301-0300 for final determination.

\section{9-12. Request for PEB evaluation}

a. RC Soldiers with non-duty related medical conditions who are pending separation for failing to meet the medical retention standards of chapter 3 of this regulation are eligible to request referral to a PEB for a determination of fitness. Because these are cases of RC Soldiers with non-duty related medical conditions, MEBs are not required and cases are not sent through the PEBLOs at the MTFs. Once a Soldier requests in writing that his or her case be reviewed by a PEB for a fitness determination, the case will be forwarded to the PEB by the USARC Regional Support Command or the AHRC Command Surgeon's office and will include the results of a medical evaluation that provides a clear description of the medical condition(s) that cause the Soldier to not meet medical retention standards.

$b$. All obligated RC participants in the Health Professions Scholarship Program (HPSP) or Financial Assistance Program (FAP) with non-duty related medical conditions who are pending separation for failure to meet medical retention standards of chapter 3 of this regulation will receive a mandatory referral to a PEB for a fitness determination. Once an HPSP or FAP participant is mandatorily referred to a PEB, the case will be forwarded to the PEB by the USARC Regional Support Command or the AHRC Command Surgeon's office and will include the results of a 
medical evaluation that provides a clear description of the medical condition(s) that cause the Soldier to not meet medical retention standards.

\section{9-13. Disposition of Reservists temporarily disqualified because of medical defects}

a. Normally, Ready or Standby Reservists temporarily disqualified because of a medical defect will be transferred to the Standby Reserve inactive list (AR 140-10). Transfer will be made if-

(1) The Soldier is not required by law to remain a member of the Ready Reserve.

(2) The Soldier is currently disqualified for retention in an active USAR status.

(3) The condition is considered to be remediable within 1 year from the date disqualification was finally determined.

$b$. When determined by the Commander, AHRC, to be in the best interest of the service, temporarily disqualified Reservists may be transferred to or kept in the Standby Reserve for 1 year. This will not be done if the Reservist requests discharge from the USAR or transfers to the Retired Reserve.

c. Reservists who by law must remain members of an RC and whose medical defects are considered to be remediable within 1 year from the date of disqualification will be kept in an active status for 1 year. These reservists will be reassigned to the USAR control group (standby).

$d$. Reservists who are temporarily disqualified will be examined no later than 1 year from the date of transfer. Those found qualified will be transferred back to the USAR status they held before they were disqualified. See AR 140-10, AR 135-175, and AR 135-178 for disposition of those found disqualified.

\section{9-14. Annual dental examinations}

a. Members of the Selected Reserve shall receive an annual oral evaluation to determine their dental classification. This annual oral evaluation will be recorded in MEDPROS at Point of Service (POS).

$b$. An annual oral examination shall be performed according to DOD Guidelines for a Periodic Oral Evaluation or the American Dental Association (ADA) procedure code D0120. This examination will consist of a clinical evaluation of the oral cavity supported by bitewings and a panographic x-ray.

(1) The frequency of prescribing radiographs is based upon the clinical judgment of the Soldier's dentist and existing practice guidelines. (for example, HHS Publication No. FDA 88-8273). Radiographs shall be of diagnostic quality, properly identified, dated and placed in the military dental record.

(2) A panographic radiograph of adequate quality for diagnostic and forensic identification purposes is required in the dental record. There is no time requirement on updating panographic radiographs. However, the panographic radiograph must adequately represent the current oral condition of the Soldier. Soldiers shall have a panographic $\mathrm{x}$-ray taken during initial dental processing. A new panographic x-ray shall be taken after extensive dental treatment.

(3) Digital x-rays are acceptable if they are a JPEG file and can be printed with approximately the size, resolution and diagnostic quality of a regular x-ray.

$c$. When the annual oral examination is performed by a civilian dentist, the examination shall be documented on a DD Form 2813 (Department of Defense Active Duty/Reserve Forces Dental Examination). Authorized medical/dental personnel will validate each examination and ensure accurate recording of the dental information on the DD Form 2813, SF 603 (Health Record - Dental) and SF 603A (Health Records - Dental Continuation) is in the dental record. An entry on the SF 603/603A must include a statement indicating the use of DD Form 2813 as verification of dental examination and the examination information. Every effort should be made for the civilian dentist to provide copies of dental radiographs used in the examination process.

$d$. Personnel performing the annual oral examination have an obligation to inform the Soldier if he/she observe or are apprised of any signs or symptoms for which the Soldier should obtain further evaluation or dental care.

$e$. Military dental record requirements: (No dental record is considered complete unless the documentation is complete and in the proper order as outlined in TB MED 250)-

(1) Military dental record jacket DA Form 5570 (Health Questionnaire for Dental Treatment);

(2) DD Form 2005 (Privacy Act Statement Health Care Records);

(3) SF 603/603A;

(4) DD Form 2813;

(5) Health Insurance Portability and Accountability Act notice of privacy practices acknowledgment label. The HIPPA notice of privacy practices is not a required dental record document by the Army Reserve. However, the HIPPA notice of privacy practices form will be provided to all Army Reserve Soldiers during their first encounter with the Active Component Dental system at the dental treatment facility or at the mobilization station. 
Chapter 10

Army National Guard

\section{0-1. General}

This chapter sets basic policies, standards, and procedures for medical examinations and physical standards for the ARNG/ARNGUS. The Clinical Section, Office of the Chief Surgeon, (NGB-ARS), is the office responsible for management of all issues pertaining to this chapter.

\section{0-2. Application}

This chapter applies to all ARNGUS Soldiers even when administered or operating in their status as members of the ARNG.

\section{0-3. Medical standards}

a. Chapter 2 standards apply to all initial enlistments, inductions, and appointments.

$b$. See AR 135-18 for the medical standards for entry into the AGR program.

c. Chapter 3 standards apply to retention in the ARNG/ARNGUS.

\section{0-4. Entry into AGR (Title 10/32) Program}

a. Soldiers who apply to enter the AGR (Title 10/32 Program) must meet chapter 3 medical retention standards. Pregnancy is not a disqualifying condition.

$b$. There are no waivers for entry into the AGR Program in accordance with paragraphs 2-1.d, and 2-2.6 and table $2-3$ in AR 135-18.

\section{0-5. Active duty for more than 30 days (other than Title 10/32 AGR)}

Prior to initiating active duty orders for more than 30 days, the National Guard Soldier must have a valid periodic health assessment (within one year) and must have no outstanding medical issues that require followup, to include a temporary profile (DA Form 3349). If the PHA is expected to expire during the deployment or time of activation, a new PHA must be completed within 60 days prior to the start of the period of active duty.

\section{0-6. Re-entry on active duty or FTNGD}

a. A Soldier may re-enter active duty, if the break in active duty service is less than 180 days from a previous period of active duty, by completing the PHA process and meeting the medical retention standards published in Chapter 3 of this regulation. The break in service must be for non-medical reasons. If the break in service was for medical reasons, a Chapter 2 physical examination must be completed.

$b$. All female Soldiers will be required to undergo pregnancy testing within 15 days prior to initiation of any active duty or any type of full time National Guard Duty (FTNGD) exceeding 30 days (except entry into the AGR program). Standard pregnancy tests performed by an accredited medical laboratory are acceptable. Pregnancy is a disqualifying factor for entry onto any active duty greater than 30 days except as noted.

\section{0-7. Applications for Federal Recognition}

Applications for Federal Recognition will include a current DD Form 2808 and DD 2807-1, within 2 years of the board action. Report of Medical Examination must indicate that Soldier meets the standards of chapter 2 for initial appointment, or has received a waiver from the approving authority.

\section{0-8. General officer medical examinations}

a. All ARNG/ARNGUS general officers will undergo a medical examination every 2 years, within 3 calendar months before the end of the officer's birth month. Examinations will be accomplished at any active MTF capable of completing these examinations or through nationally approved contractors capable of completing a medical examination.

$b$. All general officers will complete a PHA every 2 years, on the year opposite the medical examination, within 3 calendar months before the end of the general officer's birth month. (See para 8-20.) The PHA may be completed at any Active Army (AA) or RC MTF capable of completing a PHA. If the privileged provider completing the PHA indicates a need for further evaluation or medical treatment, the general officer will be referred to his/her civilian medical provider.

c. A copy of each completed physical examination or PHA will be forwarded to Chief, National Guard Bureau, ATTN: NGB-GO-AR, Room 2D366, The Pentagon, Washington, DC 20310-2500. NGB-GO-AR is responsible for forwarding completed general officer physical examinations to NGB-ARS for medical review.

$d$. Physical examinations for promotion to general officer will be obtained at Active Army MEDDAC or MEDCEN facilities, within the 6 months prior to the date of the convening selection board. 


\section{0-9. Immunizations}

a. Immunization records will be reviewed and required immunizations will be administered in accordance with AFJI 48-110/AR 40-562/BUMEDINST 6230.15/CG COMDTINST M6230.4E. For Army Special Operations, USASOC Supplement 1 to AFJI 48-110/AR 40-562/BUMEDINST 6230.15/CG COMDTINST M6230.4E applies.

b. All administered immunizations will be immediately documented in MEDPROS and on DD Form 2766. MEDPROS is the HQDA standard for tracking all individual medical readiness indicators for the all COMPOS.

\section{0-10. Periodic medical examinations}

a. The periodic (every 5 years) medical examination has been replaced by the annual requirement for a PHA. (See para 10-8 for periodic examinations for general officers.) The annual PHA does not rescind the requirements for medical examinations for specific military training programs/schools. Cardiovascular screening will be accomplished at the first regularly scheduled PHA at age 40 years.

$b$. The requirements for physical examinations for schools, for commissioning or appointment, or other special purposes remain the same.

c. Flying personnel examinations will be in accordance with chapters 4 and 6 of this regulation and USAAMA policy and guidance.

\section{0-11. Waivers}

a. Chapter 3 medical retention standards are not waiverable for induction or accession.

(1) ARNG/ARNGUS Soldiers who do not meet chapter 3 retention medical standards will be processed in accordance with AR 600-60 (MMRB), AR 40-400 (MEB), and AR 635-40 (PEB) (as appropriate).

(2) Final determination of medical qualification will be made by the Chief, NGB (NGB-ARS), except where the authority for determination has been delegated to the State Adjutants General or reserved to the Active Army.

$b$. Requests for waivers will include a detailed medical evaluation or consultation concerning the physical defect, and complete justification for the request for waiver. Only waivers submitted through the Guard Electronic Medical Records (GEMR) system (or other designated electronic system) will be accepted for review by NGB-ARS. The justification will include statements indicating service experience, MOS or position to be placed in, any known specific hazards of the position, the benefit expected to accrue from the waiver, and a recommendation of the State Surgeon. A waiver will not be recommended for medical conditions that are subject to complications or aggravation by reason of military duty.

c. Waivers for aviators, FSs, ATCs, and flight medical aidman, and final determination of medical fitness for flying duty will be made by the Chief, NGB (NGB-AVN-OP), with consideration of recommendations made by the Director, USAAMA, Fort Rucker, AL, in accordance with chapters 4 and 6 of this regulation.

d. Waivers for initial training in Airborne, Ranger, Special Forces, Military Free Fall (MFF), and Diving will not be approved/granted except on the recommendation by the Commander of the appropriate proponent school.

\section{0-12. Profiling}

a. All profiles (temporary and permanent) will be documented in the Medical Operational Data System (MODS) within the Medical Non-Deployable Module (MND) or any other approved electronic profiling database system.

$b$. Profiles will be accomplished in accordance with chapter 7 with the additional requirement that all permanent profiles (1-4) must have two signatures.

c. The State Surgeon or physician designee shall be the profile approval authority (see para 7-6c) for their respective state.

\section{0-13. Individual responsibility}

a. Each ARNG/ARNGUS Soldier is individually responsible for the maintenance of his or her medical, physical, and mental fitness. This includes correcting remediable defects, avoiding harmful habits, and weight control.

$b$. The maintenance of good strength and aerobic conditioning is of prime importance to the modern Soldier. The completion of the APFT and the ability to perform the Soldier's MOS duties are the minimum level of fitness expected from the ARNG/ARNGUS Soldier (see FM 21-20).

\section{0-14. Significant incident reporting responsibility}

$a$. Soldiers' responsibilities include seeking medical advice quickly when they believe their physical well-being is in question. Any hospitalization, significant illness, or disease that occurs when not on duty will be reported to the unit commander or first sergeant at the earliest possible opportunity and, in all cases, before initiating the next period of training.

$b$. Documentation of significant medical events which have occurred since the last period of duty or which may limit duty performance should be provided to the unit administrator for inclusion into the Soldier's health record before the next period of duty. A profile assessment by a military provider should also occur before the next period of duty. 
c. Documentation will also be placed into MODS, the Health Readiness Record (HRR) or other designated electronic database as a permanent electronic record of any significant medical events.

\section{0-15. Duty restrictions}

a. Any recommendation for restricted activity that has been made by a private physician will be reported in writing, before performing any duty.

$b$. It is the individual Soldier's responsibility to report any medical problems immediately to the chain of command and to comply with medical restrictions. Commanders will honor the private physician's recommendations until the Soldier is evaluated by a military provider, and a recommended course of action is determined by a profiling officer (see para 7-6).

c. Soldiers and commanders will abide by the medical restrictions and limitations documented on any profile (DA Form 3349) issued.

\section{0-16. Authorization for examinations}

a. Examination authorization letter. Soldiers entitled to medical examinations will be given a letter of authorization by the appropriate commander in accordance with instructions issued by the State Adjutant General. The letter will cite the examinee's name, grade, social security number, organization, purpose of the examination, and other instructions as appropriate regarding payment for the examination and distribution of the completed medical examination.

b. Issuing of orders for examinations. Issuing of orders for examinations. Soldiers undergoing examinations are to be placed on orders if not otherwise in a duty status at the time of the examination.

c. Travel expenses. Travel at Government expense will be authorized if the examination facility is outside of the established local commuting area of the Soldier's residence. The examination should be scheduled so that travel, examination, and return home can be accomplished in 1 day. If additional time is required, the Soldier will be reimbursed for meals and lodging in accordance with Joint Federal Travel Regulation (JFTR). Government meals and lodging will be used if available. A certificate of non-availability must be submitted with claims for reimbursement.

$d$. Medical readiness funds are not authorized to be used for payment of travel and per diem for medical appointments or examinations.

\section{0-17. Examination authorities}

a. Nonprior service and prior service disability separated/retirement applications.

(1) Applicants who are not prior service, or who have had medical, physical, or disability separations/retirements from prior service, or who are Soldiers of the ARNG/ARNGUS who re-enter active duty under the split training option, or who are ARNG/ARNGUS Soldiers who re-enter active duty to complete IDT will be examined only at MEPS or other authorized agency. In cases of applicants who have been previously separated for medical reasons, all prior service medical documentation, records, and medical separation board proceedings will be made available to the MEPS prior to scheduling the examination.

(2) Applicants who have a service-connected disability as determined by the VA, even though not separated for medical reasons, will be restricted to MEPS processing. VA disability determination proceedings will be made available to MEPS prior to scheduling the examination.

b. AGR/other full time duty, fitness for duty/physical profile board determination examinations.

(1) Fitness for duty of AGR and other active duty ARNGUS Soldiers will be accomplished only at Active Army MTFs.

(2) Permanent profiles issued at other than Army facilities will be submitted to the overseeing Army MTF or NGB-ARS, together with all pertinent examination and treatment records, for review, approval, and translation to Army standards.

c. Other agencies authorized to perform examinations. All other medical examinations may be accomplished by any of the following components, agencies, or civilian physicians, in order of priority. AGR will use Active Army facilities, if available in reasonable commuting distance to duty location.

(1) ARNG/ARNGUS medical staff as outlined in paragraph 8-7a.

(2) Other military medical units or facilities, ARNG/ARNGUS, Active Army, or other RC having the technical capability of performing the examinations.

(3) MEPS, on a space available basis.

(4) VA medical facilities.

(5) United States Public Health Service facilities.

(6) Contracted civilian physicians legally licensed to practice medicine in the State concerned.

\section{0-18. Examination review requirements/quality assurance}

Examinations accomplished at facilities other than MEPS and Active Army facilities will be reviewed by the State Approving Authority or Physician Designee for quality assurance, to include AGR personnel physical examinations for other than initial accession into the AGR program. The reviewer will ensure the PULHES profile is in accordance with 
chapter 7 and table $7-1$, that the DD Form 2808 is in accordance with chapter 8 , and that medical standards used to qualify or disqualify the applicant or Soldier are in accordance with the applicable chapter (for example, chap 2 or chap 3 ) for the program or purpose for which the examination was completed. The purpose of examination must be clearly noted. The examination must be approved and signed by the reviewing officer.

\section{0-19. Scope of medical examinations}

a. Change from original purpose of examination. In the event a physical examination is to be employed for other than the original stated purpose for which it was performed, the examining privileged provider will enter a note in block 73. Any additional procedures after the original date of the examination will be entered in the appropriate block on the DD Form 2808 and initialed and dated by the exam provider. An entry will be made in block 73 listing all additional items accomplished, dated, signed, and stamped with the provider's identification information.

(1) When a physical examination has been modified from its original purpose, the date of the modification cannot exceed the validity time prescribed in paragraph 8-14. (Example: Physical exam performed in January 2002. In January 2004 the examination was modified for Ranger School. This would be invalid because the validity time for a Ranger School exam is 18 months.)

(2) The following is an example of an acceptable entry in block 73: "DATE. This examination has been reviewed by chapter 2 standards. All required items completed and listed appropriately. PULHES: 111121. Individual Qualified or Not Qualified. Signature."

b. Required specialty consultations. If additional examinations or specialty consultations beyond the capabilities of the examining facility are required, the State Medical Detachment will be notified. An SF 513 (Medical Record (Continuation Sheet)) will be completed by the requesting physician and furnished to the Soldier. The Soldier will be required to provide the completed SF 513 to the State Medical Detachment for completion of required consultations. Consultations and further examinations will be at the Soldier's expense unless otherwise noted.

c. Occupational Medicine Exams. DOD 6055.5-M, Occupational Medical Surveillance Manual, outlines minimum standard for establishing medical surveillance programs. Per DOD 6055.5-M paragraph C1.5.2.1, "Local occupational medical personnel establish examination content and frequency based on an understanding of the job demands, exposures to the workers, the medical effects of specific exposures, the impact of specific medical conditions on job performance and safety and legal and regulatory requirements."

\section{0-20. Report of medical examinations}

DD Form 2808 and DD Form 2807-1 and all continuation pages, and consultations will be submitted as follows:

a. The original will be forwarded directly to Medical Records Custodian for the following actions:

(1) A copy will be placed into the Soldier's health record.

(2) A copy will be scanned into MODS Health Readiness Record (HRR).

$b$. A copy will be maintained at the examination facility.

c. A copy will be furnished to the individual as required for schools, promotions, and other administrative actions in accordance with regulation and policy.

d. Copies will be made available for Enlistment and Re-enlistment as prescribed by NGR 600-200.

\section{0-21. Directed examinations}

The Chief, NGB, the State Adjutant General, the commanding officer of a Soldier's unit, or a medical officer may direct the Soldier to undergo a medical examination in accordance with AR 600-20 whenever, in the authority's opinion, the Soldier's medical, physical, dental or mental condition is such that an examination is indicated.

\section{0-22. Administrative information}

a. Any Soldier without a current PHA will not attend IDT or AT.

b. HIV testing will be completed in accordance with AR 600-110.

c. A special medical examination is not required for attendance at an Army service school, except as indicated below.

\section{0-23. Special examinations}

a. Command and General Staff Course (Resident) and the regular course at the United States Army War College. A MEDPROS print-out that shows that the PHA has been accomplished within the preceding 12 months will be forwarded with the school application to the school proponency at NGB. Chapter 3 medical standards for retention apply for physical examination review.

b. Entry into Active Army OCS, State OCS, Warrant Officer Candidate School, and Airborne, Ranger, or Pathfinder training. A complete physical examination (DD Form 2808 and DD Form 2807-1) is required, in accordance with chapters 2, 5, and 8 of this regulation, and will be accomplished within the preceding 24 months prior to the first day of school attendance. A PHA will be accomplished within 60 days preceding the start of school. 
c. Initial flight training course. Physical examinations will be accomplished and approved in accordance with chapters 4 and 8 of this regulation prior to submission to NGB-ARO-TI.

d. Special Forces initial qualification, MFF, and Combat Diver examinations. Physical examinations will be accomplished and approved in accordance with paragraph 8-25 prior to submission to NGB-ARS.

\section{0-24. Cardiovascular Screening Program (AGR Soldiers)}

a. The CVSP for Title 10/32 AGR Soldiers will be conducted in accordance with paragraph 8-26 of this regulation with the PHA process.

$b$. Soldiers who do not obtain CVSP clearance will be medically flagged and processed through the MMRB in accordance with AR 600-60.

\section{0-25. Soldiers pending separation for failing to meet medical retention standards}

a. Not in the Line of Duty (NILOD). DODI 1332.38 states that members with non-duty related impairments are eligible to be referred to the PEB solely for a fitness determination, but not a determination of eligibility for disability benefits. Further explanation is available in TAPD-Policy Memorandum \#4, Processing Reserve Component (RC) NonDuty Related Cases. This policy memorandum outlines the procedures and requirements for processing boards on RC Soldiers with non-duty related impairments that are pending separation for medical disqualification. Determination of whether a non-duty case is forwarded to the PEB is at the request of the Soldier. The Soldier will have a completed LOD or memo that notifies him/her of non-duty related findings (Not In the Line of Duty -NILOD). The Soldier may not challenge the PEB findings in person.

(1) The Non-Duty PEB packet will include: the completed DA Form 2173 (Statement of Medical Examination and Duty Status) showing NILOD findings, DA Form 5889-R (PEB Record of Transmittal) completed in accordance with TAPD-Policy Memorandum \#4, ND-PEB Checklist with the following supporting documents: Soldier Notification of Pending Separation for Medical Disqualification, Soldier's Counseling of their right to a PEB, Soldier's election Letter, DA Form 3349, Fitness For Duty (FFD) examination with appropriate civilian/military consults, Commander's Statement on Duty Performance, DA Form 705 (Army Physical Fitness Test Scorecard) and any other evidence that the Soldier elects to provide.

(2) The MILPO is responsible for notifying the Soldier, in writing, that his/her injury is NILOD and that he/she is pending separation for a medical disqualifying condition. The notification will also advise the Soldier that he/she has the right to prepare a Non-Duty PEB packet for a fitness determination.

(3) The State Surgeon is responsible for completing the following: Physical Profile, a Fitness for Duty examination and obtaining the appropriate civilian consults from the Soldier (non-Duty related cases are the Soldier's responsibility and he/she must provide the appropriate medical consults from his/her civilian physician).

(4) The Commander is responsible for counseling the Soldier and completing a Commander's Letter of Duty Performance.

(5) The Soldier is responsible to request his/her packet be submitted to the PEB for adjudication. The Soldier is responsible for preparing his/her packet for submission to the PEB.

$b$. In the Line of Duty (ILOD). Soldiers pending separation for ILOD injuries or illnesses will be processed in accordance with AR 40-400 and AR 635-40.

\section{0-26. Annual dental examination}

a. Members of the Army National Guard shall receive an annual oral evaluation to determine their dental classification. This annual oral evaluation will be recorded in MEDPROS at Point of Service (POS).

$b$. An annual oral examination shall be performed according to DoD Guidelines for a Periodic Oral Evaluation or the American Dental Association (ADA) procedure code D0120. This examination will consist of a clinical evaluation of the oral cavity supported by bitewings and a panographic x-ray.

(1) The frequency of ordering radiographs is based upon the clinical judgment of the Soldier's dentist and existing practice guidelines. (e.g. HHS Publication No. FDA 88-8273). Radiographs shall be of diagnostic quality, properly identified, dated and placed in the military dental record.

(2) A panographic radiograph of adequate quality for diagnostic and forensic identification purposes is required in the dental record. There is no time requirement on updating panographic radiographs. However, the panographic radiograph must adequately represent the current oral condition of the Soldier. Soldiers shall have a panographic x-ray taken during initial dental processing. A new panographic x-ray shall be taken after extensive dental treatment.

(3) Digital x-rays are acceptable if they are a JPEG file and can be printed with approximately the size, resolution and diagnostic quality of a regular x-ray.

c. When the annual oral examination is performed by a civilian dentist, the examination shall be documented on a DD Form 2813. Authorized medical/dental personnel will validate each examination and ensure accurate recording of the dental information on the DD Form 2813 and SF 603/603A is in the dental record and in DENCLASS. An entry on the SF 603/603A must include a statement indicating the use of DD Form 2813 as verification of dental examination 
and the examination information. Every effort should be made for the civilian dentist to provide copies of dental radiographs used in the examination process.

$d$. Personnel performing the annual oral examination have an obligation to inform the Soldier if he/she observes or are apprised of any signs or symptoms for which the Soldier should obtain further evaluation or dental care.

$e$. Military dental record requirements: (No dental record is considered complete unless the documentation is complete and in the proper order as outlined in TB MED 250).

(1) Military dental record jacket DA Form 5570 (envelope);

(2) DD Form 2005 (Privacy Act Statement Health Care Records);

(3) SF 603/603A;

(4) DD Form 2813;

(5) Health Insurance Portability and Accountability Act notice of privacy practices acknowledgment label. The HIPPA notice of privacy practices is not a required dental record document by the ARNG. However, the HIPPA notice of privacy practices form will be provided to all ARNG Soldiers during their first encounter with the Active Component Dental system at the dental treatment facility or at the mobilization station.

\section{0-27. Physical inspections prior to annual training}

a. Unit commanders are responsible for individual inspection of all personnel under their command immediately prior to departure for AT (normally within 72 hours).

$b$. As a minimum, this screening will consist of-

(1) Confirmation that a current PHA is on hand for each Soldier scheduled to attend AT.

(2) Physical observation for any outward signs of existing injury or disease, including bandages, splints, casts, use of crutches, braces, or other orthopedic devices.

(a) Any Soldier that has not previously been evaluated or exhibits signs of an obvious physical, psychiatric, or dental condition that is likely to interfere with or be aggravated by AT will be required to be evaluated by a military medical officer, including the completion of a new PHA before being allowed to depart for AT.

(b) If this evaluation results in a determination of a significant category change, the Soldier may not attend AT until cleared.

c. The commander will certify in the remarks section of unit DA Form 1379 (U.S. Army Reserve Components Unit Record of Reserve Training) that the screening in b above took place before unit annual training, and will ensure that this certification includes his or her name, unit, and date. This statement will read: "I, the (Commander) of (Unit) performed a physical inspection of each Soldier present and attending annual training on (Date), prior to departing for unit annual training."

\section{Chapter 11 Individual Medical Readiness Standards}

\section{1-1. General}

This chapter implements Department of Defense Instruction 6025.19 and supplements the information provided in AR $220-1$.

\section{1-2. Purpose}

a. The purpose of the chapter is to establish measurable medical elements as components of Individual Medical Readiness (IMR). Compliance will be monitored and reported on the AMEDD Balanced Scorecard. This compliance report includes all IMR data with the exception of Pap smear, Class 3 dental work, and additional series vaccinations.

$b$. The IMR program equips unit commanders and PCMs with the tools to monitor the IMR status of their personnel and patients, resulting in a healthy and fit fighting force medically ready to deploy.

$c$. The DoD requires quarterly reporting of the IMR status of Active and Selected Reserve members, except those Soldiers who have not completed initial military training (IMT) and follow-on technical skills training or others who are unavailable to deploy (for example, recruiters, ROTC cadre, students in deferred status pursuing advanced academic degrees).

$d$. The IMR program enables commanders and staffs at all levels to analyze and address key unit status medical indicators/elements.

\section{1-3. Responsibilities}

a. The unit status report (USR) is a commander's report. Unit commanders are solely responsible for the accuracy of the information and data they enter into their reports. Unit Commanders are responsible for monitoring their Soldiers' IMR status and ensuring compliance. Unit medical assets, when available, are primarily responsible for supporting medical readiness. 
b. Regional Medical Command (RMC) Commanders will ensure MTFs provide the necessary medical care to Soldiers to ensure they meet the IMR standards. MTFs will assist commanders with supporting medical assets to maximize the number of personnel classified as fully medically ready (FMR) when unit medical assets are not available to supply necessary services.

(1) The MTFs providing medical care to Soldiers during Basic Combat Training will update the IMR status during initial in-processing.

(2) The MTFs will not refuse IMR related appointments for AC Soldiers enrolled in TRICARE Prime Remote. Soldiers on Active Duty status who are assigned to TRICARE Prime Remote may utilize the Reserve Health Readiness Program (RHRP) once a voucher process is established.

(3) All Soldiers out processing through an MTF are to be FMR, if possible, prior to clearing the MTF.

\section{1-4. Individual Medical Readiness elements}

The USR Personnel level is based on that portion of a unit's required strength that is available for deployment/ employment with the unit to accomplish its wartime mission. The individual medical readiness (IMR) elements are one portion of the personnel level of the USR (see AR 220-1). The IMR consists of the following elements:

a. Health assessment.

(1) The Periodic Heath Assessment (PHA) will be considered the health assessment (HA) and is considered current if it has been less than 15 months since the last PHA. During months 1 through 11, the Soldier is considered "Green". From months 12 to 15, the Soldier is considered "Amber." At the 15th month since the last PHA, the Soldier will be considered "Red" (See para 8-20 of this regulation for PHA requirements).

(2) The HA consists of the date of the HA, height, weight, PULHES, and potential for deployablility within 6 months.

(3) The results of the PHA will be directly entered at the Point of Service (POS) into MEDPROS. MEDPROS PHA information (PULHES, profile codes, date of PHA and for RC, height and weight) will automatically update the information in the Total Army Personnel Database (TAPDB). The PULHES will be updated to reflect permanent profiles only.

(4) Deployed Soldiers are exempt from the PHA requirement until 90 days after returning from deployment. For the time period of 90 days until 180 days, the Soldier will be considered "Amber." If the Soldier does not update his PHA by the 180th day after his return from deployment, he will be considered "Indeterminate" and "Gray" in MEDPROS.

$b$. Deployment limiting medical conditions. Soldiers who will not deploy with the unit for various medical reasons will be considered medically not ready and categorized as "Red" in MEDPROS. Soldiers with deployment-limiting conditions are-

(1) Active duty Soldiers admitted as an inpatient to an MTF. These Soldiers are categorized as "absent sick status."

(2) Soldiers who are pregnant. Testing for pregnancy is not a routine requirement. Positive pregnancy tests must be manually posted into MEDPROS by the medical staff at the location where the test was obtained. A pregnant Soldier is not deployable and categorized as "Red" in MEDPROS.

(3) Soldiers with permanent (P3/P4) profiles unless they have been cleared for deployment by a MOS Medical Retention Board (MMRB) and/or have been found "fit for duty" by a PEB without any deployment limitations (or medically cleared to mobilize-RC only).

(a) Soldiers who completed appropriate board actions (MMRB, PEB) must have the appropriate Profile Code (from box 2 on DA Form 3349) entered into MEDPROS.

(b) Code "W" for MMRB complete, code "X" for COAD or COAR after the Soldier was found "unfit" by a PEB, or code "Y" after the Soldier was found "Fit for Duty" by a PEB. (See table 7-2 for complete list of codes).

(c) All physical profiles exceeding 30 days duration will be entered electronically in either AHLTA or the Medical Non-deployable Module (MNM) of MODS to improve visibility and management of the Soldiers with profiles.

(4) Personnel with a temporary (T3/T4) profile that cannot be medically cleared for deployment (includes prenatal and postpartum profiles according to para 7-9). The appropriate Profile Code(s), from box 2 on DA Form 3349, must be entered into MEDPROS.

(5) Soldiers assigned to Warrior Transition Units.

c. Dental readiness. (See AR 40-3, para 6-5 for complete information.)

(1) Dental Class 1. Soldiers with a current dental examination, who do not require dental treatment or reevaluation. Class 1 Soldiers are worldwide deployable and classified as medically ready and "Green" in MEDPROS.

(2) Dental Class 2. Soldiers with a current dental examination, who require non-urgent dental treatment or reevaluation for oral conditions which are unlikely to result in dental emergencies within 12 months. However, Dental Class 2 Soldiers still have active dental disease that will eventually require treatment. Dental Class 2 Soldiers are worldwide deployable, considered medically ready, and classified as "Green" in MEDPROS.

(3) Dental Class 3. Soldiers who require urgent or emergent dental treatment. Dental Class 3 Soldiers are normally considered medically not ready and are classified as "Red" in MEDPROS.

(4) Dental Class 4. Soldiers who require dental examinations. This includes Soldiers who require annual or other 
required dental examinations and Soldiers whose dental classifications are unknown. Dental Class 4 Soldiers are normally considered to be medically not ready and are classified as Indeterminate status and "Red" in MEDPROS.

d. Immunizations.

(1) Only the routine mandatory immunization (IM) profile (adult panel) is tracked and reported as part of the USR (see AR 40-562).

(2) Mandatory IMs for all Army personnel include-

(a) Hepatitis A -2 doses at 0 and 6-12 months after the first injection or the Hepatitis A/Hepatitis B combined vaccine (Twinrix) -3 doses at 0,1 month and 6 month intervals.

(b) Tetanus/Diphtheria (Td) booster every 10 years or Tetanus/diphtheria acellular pertussis (Tdap) as a one time booster.

(c) An annual influenza immunization.

(3) All other adult immunizations will be maintained as per AR 40-562.

(4) Other immunizations will be given dependent on risk assessment for the country of deployment or Soldier's physical condition.

(5) The MTF or medical personnel who administered the vaccine to the Soldier are responsible for ensuring entry of the immunization data into MEDPROS or AHLTA. Unit commanders are responsible for ensuring that the Soldiers report to immunization clinics to obtain required immunizations.

(6) Soldiers are considered medically ready and "Green" if current on all mandatory immunizations. If the Soldier is missing one or more mandatory immunizations, the Soldier will be considered medically not ready and categorized as "Amber" in MEDPROS.

e. Deoxyribonucleic acid. A DNA specimen is obtained once and is on file at the Armed Forces DNA Repository.

(1) Soldiers with a DNA sample on file and recorded in MEDPROS are considered medically ready and categorized as "Green" in MEDPROS.

(2) Soldier will be considered as medically not ready and categorized as "Red" in MEDPROS if there is no evidence that a DNA specimen was drawn ("D" in MEDPROS) or if there is no evidence that a specimen is on file at the Armed Forces DNA Repository.

(3) If the DNA on file category in MEDPROS is blank, the Soldier is considered medically not ready and categorized as "Amber" in MEDPROS.

f. Current Human Immune Deficiency Virus. The Human Immune Deficiency Virus (HIV) antibody test as required by AR 600-110.

(1) Soldiers with a current HIV antibody test (and received at Army Medical Surveillance Activity (AMSA)) within 2 years are considered medically ready and categorized as "Green" in MEDPROS.

(2) Soldiers without a current HIV test (MEDPROS will default to incomplete after 30 days overdue) are considered medically not ready and categorized as "Red" in MEDPROS.

g. Hearing readiness.

(1) The Defense Occupational Environmental Hearing Readiness Application-Hearing Conservation (DOEHRS-HC) audiometer is the only authorized audiometer for conducting and recording DD Form 2215 (Reference Audiogram) and DD Form 2216 (Hearing Conservation Data).

(2) All military personnel will receive a DD Form 2215 at IET prior to noise exposure. (See DA Pam 40-501 for more information.)

(3) All Soldiers assigned to a TOE unit or who have a PROFIS assignment to a TOE unit must complete hearing readiness requirements to include an annual DD Form 2216.

(4) Hearing Readiness Categories are as follows:

(a) Hearing Class 1. Soldier has a current, within 12 months, DOEHRS-HC audiogram. Unaided hearing is within H-1 profile standards (see table 7-1). Soldier will be considered medically ready and categorized as "Green" in MEDPROS.

(b) Hearing Class 2. Soldier has current, within 12 months, DOEHRS-HC audiogram. Unaided hearing is within H2 or H-3 profile standards and Soldier has a permanent profile recorded in MEDPROS for hearing. If Soldier requires hearing aid(s), he/she must have prescribed hearing aid(s) and a 6-month supply of batteries. Soldier will be considered medically ready and categorized as "Green" in MEDPROS.

(c) Hearing Class 3. Soldier has current, within 12 months, DOEHRS-HC audiogram. Unaided hearing is within H2 or H-3 profile standards but the Soldier requires a complete audiological evaluation (Speech Recognition in Noise Test (SPRINT) is required) to document permanent hearing profile on DA Form 3349 or needs prescribed hearing aid(s). If the Soldier meets medical retention standards of chapter 3, AR 40-501, than he/she requires a MMRB. Soldier will be considered medically not ready and categorized as "Red" in MEDPROS.

(d) Hearing Class 4. Soldier requires a DOEHRS-HC audiogram. Soldier does not have a reference baseline audiogram or a current periodic audiogram. Soldier will be considered medically not ready and categorized as "Red" in MEDPROS. 
h. Vision readiness. Visual acuity testing will not be done with the Soldier wearing contact lenses. The vision readiness categories are as follows:

(1) Vision Class 1. Soldier has corrected vision of 20/20 (with both eyes open), either with best spectacle correction or without spectacles. If spectacles are required, Soldier has a record of spectacle prescription recorded in MEDPROS. Soldier will be considered medically ready and categorized as "Green" in MEDPROS.

(2) Vision Class 2. Soldier has corrected vision between 20/25 and 20/40 or an accession waiver for vision worse than 20/45 (with both eyes open), either with best spectacle correction or without spectacles. If spectacles are required for the Soldier to achieve this visual acuity, the Soldier has a record of a spectacle prescription recorded in MEDPROS. Soldier's vision may require an update of their spectacle prescription and a referral to Optometry is recommended. Soldier will be considered medically ready and categorized as "Green" in MEDPROS.

(3) Vision Class 3. Soldier has best corrected vision worse than 20/45, or no spectacle prescription on record (if required), or the spectacle prescription is older than 4 years. Referral to Optometry is mandatory. Soldier will be considered as medically not ready and categorized as "Amber" in MEDPROS.

(4) Vision Class 4. Soldier has not completed a visual acuity screening the past 365 days or the vision data is incomplete. Soldier will be considered as medically not ready and categorized as "Amber" in MEDPROS.

i. Women's readiness.

(1) Female Soldiers are required to have an annual pap smear unless they meet one of the following criteria:

(a) If the Soldier is 30 years of age or older, has no past history of dysplasia and she has had 3 consecutive normal pap smears. These Soldiers are required to have a pap every 3 years.

(b) If the Soldier has had a hysterectomy for reasons other than cervical dysplasia or cancer, she is not required to have pap.

(2) Female Soldiers 25 years of age or younger are required to have annual Chlamydia testing.

(3) Women's Health Readiness Categories are as follows:

(a) Women's Class 1. Soldier has normal cervical cytology within 1 year; or those Soldiers, 30 years of age or older, who meet the criteria in (1)(a) and have had a normal cytology within 3 years. Soldier will be considered medically ready and categorized as "Green" in MEDPROS.

(b) Women's Class 2. This class includes Soldiers, 20 years of age and younger, who have a history of abnormal cervical cytology to include cytology showing Atypical Squamous Cells of Unknown Significance (ASC-US); Human Papilloma Virus (HPV) typing negative or positive for oncogenic HPV; Low-grade Squamous Intraepithelial Lesion (LGSIL) or Cervical Intraepithelial neoplasia (CIN) 1 (mild). This class also includes those Soldiers of any age with abnormal cervical cytology which has been fully evaluated and/or treated and they have been cleared for deployment by a provider credentialed in women's health. Soldier will be classified as medically ready and categorized as "Green" in MEDPROS.

(c) Women's Class 3. Soldier's most recent cervical cytology is abnormal and requires further evaluation by a gynecologist. For Soldiers 20 years of age and younger, the following cervical cytology results require further evaluation: High-grade Squamous Intraepithelial Lesion (HGSIL); CIN 2 (moderate), CIN 3 (severe), Carcinoma in situ (CIS), Adenocarcinoma in situ (AIS), AGC, or invasive cancer. For Soldiers over the age of 20 the following cervical cytology results require further evaluation: ASC-US; HPV Positive; LGSIL; HGSIL; any CIN grade; CIS; AIS; Atypical Glandular Cells (AGC); or invasive cancer. Soldier will be considered medically not ready and categorized as "Red" in MEDPROS.

(d) Women's Class 4. Soldier has not had a pap smear within the last 365 days; unless she falls in the categories described in paras (1)(a) and (b) of Women's Readiness. Soldier will be considered medically not ready and classified as "Red" in MEDPROS.

(4) The PCMs and unit commanders should counsel all female Soldiers about women's health during deployments. Unit commanders will ensure female Soldiers have access to a copy of "Female Guide to Readiness" available at http:// chppm-www.apgea.army.mil/documents/TG/TECHGUID/TG281Draft29SepFinal.pdf.

(5) The PCMs should counsel all female Soldiers up to age 26 about the benefits of the HPV vaccine and offer the vaccine.

j. Pregnancy. Pregnancy testing should only be conducted within 30 days of deployment. A pregnant Soldier is categorized as "Red" in MEDPROS.

\section{1-5. Individual Medical Readiness categories}

After evaluating the Soldier in the 9 required elements, the Soldier will be categorized by MEDPROS into one of four medical readiness categories:

a. Medical Readiness Class 1 (MRl)

(1) All medical requirements met.

(2) Soldier is fully medically ready in all elements.

(3) Optical devices ordered.

(4) Soldiers categorized as "Green" in MEDPROS. 
b. Medical Readiness Class 2 (MR2)

(1) Medically ready within 72 hours (any deficiencies correctable during final Soldier Readiness Program (SRP)).

(2) Deficiencies may include immunizations, Dental Class 2 conditions, lack of medical warning tags, need HIV or

DNA lab tests, or optical prescription on file but eye equipment not ordered.

(3) Soldiers categorized as "Green" in MEDPROS.

c. Medical Readiness Class 3A (MR3A)

(1) Medically ready within 30 days.

(2) Deficiency may include Dental Class 3.

(3) This time frame allows for the medical treatment of abnormal screening tests.

(4) Includes deficiencies that are resourced through Transition Assistance Management Program (TAMP) for correction in alerted Selected Reserve Soldiers.

(5) Soldiers are categorized as "Red" in MEDPROS.

d. Medical Readiness Class 3B (MR3B)

(1) Medical requirements will take more than 30 days to correct.

(2) Deficiencies may include temporary profiles exceeding 30 days, and P3 or P4 profiles that require completion of a MMRB. (If the Soldier does not meet medical retention standards, Soldier requires a MEB.)

(3) Soldiers who are pregnant.

(4) Soldiers who are hospitalized (absent sick status).

(5) Soldiers found "Unfit" but continued in COAD status.

(6) Soldiers are categorized as "Red" in MEDPROS.

e. Medical Readiness Class 4 (MR4)

(1) Medical readiness requirement deficiencies are considered in an indeterminate status.

(2) Deficiencies may include:

(a) No current periodic health assessment (PHA).

(b) No current dental screen.

(3) Categorized as "Gray" in MEDPROS.

\section{1-6. Disposition of Individual Medical Readiness data}

a. The MEDPROS is the database of record for all medical readiness data elements.

$b$. All IMR data will be updated in MEDPROS for all Army personnel (all COMPOS), including deploying Department of the Army civilians, regardless of TRICARE enrollment.

c. Until bidirectional interfaces are functional, medical readiness services completed in AHLTA (with the exception of immunizations) must be updated in MEDPROS within 72 hours of completion. (This can be done through a single source sign on portal to Medical Operational Data System (MODS) that will be available with AHLTA 3.3).

$d$. The MEDPROS will automatically update the Army Status of Resources and Training System (ASORTS) database, which serves as the central registry and authorized database of record for all operational Army organizations and units. (In FY07, ASORTS will become the Defense Readiness Reporting System-Army (DRRS-A) database.) 
Appendix A

References

Section I

Required Publications

AR 12-15

Joint Security Assistance Training (JSAT) (Cited in para 6-9b.)

AR 40-3

Medical, Dental, and Veterinary Care (Cited in paras 6-4a, 6-4j(1), 8-12b(3), and 11-4c.)

AR 40-29/AFR 160-13/NAVMEDCOMINST 6120.2/CG COMDTINST M6120.8

Medical Examination of Applicants for United States Service Academies, Reserve Officer Training Corps (ROTC) Scholarship Programs, Including Two- and Three-year College Scholarship Programs (CSP), and the Uniformed Services University of the Health Sciences (USUHS) (Cited in paras 1-6c, 8-7c, 8-10a, 8-12e, and 8-15a.)

AR 40-66

Medical Record Administration and Health Care Documentation (Cited in paras 8-5a, 8-5b(2), 8-18, and 8-25b(5).)

AR 40-400

Patient Administration (Cited in paras 3-3, 6-9e, 6-12b(1), 7-4b, 8-4a, 8-8, 10-11a(1), and 10-25b.)

AR 40-562/BUMEDINST 6230.15/AFJI 48-110/CG COMDTINST M6230.4E

Immunizations and Chemoprophylaxis (Cited in paras $10-9 a$ and 11-4d.)

AR 55-46

Travel Overseas (Cited in para $5-14 g$.)

AR 95-1

Flight Regulations (Cited in para 6-11d.)

AR 95-20/NAVAIRINST 3710.1F/AFJI 10-220/DCMA INST 8210.1/AFI 10-220/COMDTINST M13020.3

Contractor's Flight and Ground Operations (Cited in para 4-31a(2).)

AR 135-18

The Active Guard Reserve (AGR) Program (Cited in paras 10-3b and 10-4.)

AR 135-100

Appointment of Commissioned and Warrant Officers of the Army (Cited in para 9-5a.)

AR 135-175

Separation of Officers (Cited in paras 3-7h, 3-35b, 9-10a, and 9-13d.)

AR 135-178

Enlisted Administrative Separations. (Cited in paras 3-7h, 3-35b, 9-10a, and 9-13d.)

AR 140-10

Assignments, Attachments, Details, and Transfers (Cited in paras 3-7h, 9-10a, 9-13a, and 9-13d.)

AR 145-1

Senior Reserve Officers' Training Corps Program: Organization, Administration, and Training (Cited in paras 8-15a and 9-2a(1).)

AR 145-2

Organization, Administration, Operation, and Support (Cited in para 9-2a(1).)

AR 385-10

The Army Safety Program (Cited in para 6-4j(4).) 


\section{AR 600-8-24}

Officer Transfers and Discharges (Cited in paras 3-3b, 3-35b, and 7-9b(3), and table 8-2.)

AR 600-8-101

Personnel Processing (In-, Out-, Soldier Readiness, Mobilization, and Deployment Processing) (Cited in para 5-14g.)

AR 600-8-105

Military Orders. (Cited in para 6-17f(2).)

\section{AR 600-9}

The Army Weight Control Program (Cited in paras 2-21a, 4-17, 4-31c, 5-9l, 5-11l, 5-11m(2), and 7-13 and tables 2-1, 2-2, and 8-1.)

\section{AR 600-85}

Army Substance Abuse Program (ASAP) (Cited in para 4-23h(2).)

\section{AR 600-105}

Aviation Service of Rated Army Officers (Cited in paras 4-2, 4-23l, 4-29, 6-2, 6-4f, 6-4, 6-8b(4), 6-10f, 6-11, 6-12b(1), 6-16, and 6-18.)

\section{AR 600-106}

Flying Status for Nonrated Army Aviation Personnel (Cited in paras 4-2 and 6-2a.)

\section{AR 600-110}

Identification, Surveillance, and Administration of Personnel Infected with Human Immunodeficiency Virus (HIV) (Cited in paras 3-7h, 4-5b, 4-33b(8), 8-12e, 8-14a(8), 10-22b and 11-4f.)

\section{AR 601-270/AFR 33-7/MCO P-1100.75A}

Military Entrance Processing Stations (MEPS) (Cited in paras 8-4a and 8-15a.)

\section{AR 608-75}

Exceptional Family Member Program (Cited in paras 5-14d and 8-25b(6).)

\section{AR 611-85}

Aviation Warrant Officer Training (Cited in para 4-2a(1).)

AR 611-110

Selection and Training of Army Aviation Officers (Cited in para 4-2a(1).)

AR 614-30

Overseas Service (Cited in para 7-9d(1).)

\section{AR 614-200}

Enlisted Assignments and Utilization Management (Cited in para 5-14g.)

\section{AR 635-40}

Physical Evaluation for Retention, Retirement, or Separation (Cited in paras 2-2c(2)(b), 3-3, 3-3b, 3-3e, 3-7h, 6-12, 8-24a, 10-11a(1), 10-25b, table 7-2, and table 8-2.)

\section{AR 635-200}

Active Duty Enlisted Administrative Separations (Cited in paras 2-2c(2)(a), 3-3b, 3-35b, 7-9b(3), 8-24a(1), and table 8-2.)

\section{DA Pam 385-90}

Army Aviation Accident Prevention Program (Cited in paras 6-4j.)

\section{DOD 6055.5-M}

Occupational Health Surveillance Manual (Cited in para 10-19c.) 
FM 3-04.301 (obsolete)

Aeromedial Training for Flight Personnel

\section{TB MED 287}

Pseudofolliculitis of the Beard and Acne Keloidalis Nuchae (Cited in para 7-3e(5). Available at http://chppm-www. apgea.army.mil/tbm.htm.)

APL series

Aeromedical Policy Letters (Cited in paras 4-1e, 4-4d, 4-5a(2), 4-6b, 4-8, 4-9, 4-10, 4-11, 4-12, 4-13, 4-15, 4-18e, 4-20a, 4-22b, 4-23, 4-27b, 4-31b, 4-32a, 4-33b, 6-2, 6-5b, 6-9b, 6-10, 6-11f, 6-12, 6-14, 6-16, 6-18 and 6-20. Available at http://usasam.amedd.army.mil/.)

\section{ATB series}

Aeromedical Technical Bulletins (Cited in paras 4-1c, 4-2e, 4-5b, 4-12, 4-15, 4-16a, 4-30, 4-31, 4-32a, 4-33b(8), 6-1c, 6-2d, 6-5b, 6-7b, 6-8a, 6-9a, 6-10e, 6-11d, 6-12, 6-13d, 6-16, 6-18, 6-20, and 8-12h. Available at http:// usasam.amedd.army.mil/.)

\section{DSM-IV}

Diagnostic and Statistical Manual of Mental Disorders, Fourth Edition, Revised, American Psychiatric Association (Cited in paras 3-30j and 4-23. This manual may be ordered at www.appi.org.)

\section{NATO STANAG 3526}

Interchangeability of NATO Aircrew Medical Categories (Cited in paras 4-1c and 6-9b. Available at http://www.epublishing.af.mil/.)

Assistant Secretary of Defense (Health Affairs) Policy Memorandum, Subject: Policy Guidance for Deployment-Limiting Psychiatric Conditions and Medications, dated 7 Nov 2006

(Cited in para 5-14f(8). Available at: http://www.dtic.mil/whs/directives/corres/ins1.html.)

\section{Section II}

\section{Related Publications}

A related publication is a source of additional information. The user does not have to read a related publication to understand this regulation. Unless otherwise indicated, DOD publications are available at http://www.dtic.mil/whs/ directives. The United States Code and the Code of Federal Regulations are available at http://www.gpoaccess.gov/.

\section{AR 40-5}

Preventive Medicine

\section{AR 40-8}

Temporary Flying Restrictions Due to Exogenous Factors

\section{AR 40-68}

Clinical Quality Management

AR 135-91

Service Obligations, Methods of Fulfillment, Participation Requirements, and Enforcement Procedures

AR 135-133

Ready Reserve Screening, Qualification Records System and Change of Address Reports

AR 140-1

Mission, Organization, and Training

AR 140-185

Training and Retirement Point Credits and Unit Level Strength Accounting Records

AR 220-1

Unit Status Reporting 
AR 350-1

Army Training and Leader Development

AR 600-8-10

Leaves and Passes

AR 600-20

Army Command Policy

AR 600-60

Physical Performance Evaluation System

AR 601-210

Active and Reserve Components Enlisted Program

AR 611-75

Management of Army Divers

AR 614-10

U.S. Army Personnel Exchange Program With Armies of Other Nations; Short Title: Personnel Exchange Program

AR 635-10

Processing Personnel for Separation

AR 670-1

Wear and Appearance of Army Uniforms and Insignia

DA Pam 40-501

Hearing Conservation Program

DA Pam 600-8

Management and Administrative Procedures

DA Pam 611-21

Military Occupational Classification and Structure

DFAS-IN Regulation 37-1

Finance and Accounting Policy Implementation. (Available at http://www.asafm.army.mil.)

DOD 7000.14-R, Vol 7A

Military Pay Policy and Procedures - Active Duty and Reserve Pay

DODD 1308.1

DOD Physical Fitness and Body Fat Program

DODI 6130.4

Medical Standards for Appointment, Enlistment, or Induction in the Armed Forces

DODI 1332.14

Enlisted Administrative Separations

DODI 6025.19

Individual Medical Readiness (IMA)

DODD 6130.3

Physical Standards for Appointment, Enlistment, and Induction

DODI 6490.03

Deployment Health 
FM 3-04.300

Flight Operations Procedures (Available at https://www.us.army.mil/suite/login/welcome.html.)

FM 21-20 (obsolete)

Physical Fitness Training (Available at https://www.us.army.mil/suite/login/welcome.html.)

MIL-PRF-680B

Degreasing Solvent (Available at http://assist.daps.dla.mil/quicksearch)

NATO STANAG 3526

Interchangability of NATO Aircrew Medical Categories (Available at http://www.e-publishing.af.mil/.)

NGR 600-200

Enlisted Personnel Management and Fiscal Year (FY) Enlistment Criteria Memorandum (ECM) (Available at the Guard Knowledge Online (GKO) https://gko.ngb.army.mil/.)

OPM Operating Manual

Qualification Standards Handbook for General Schedule Positions (Available at http://www.opm.gov/qualifications/ index.htm.)

\section{Periodic Health Assessment}

U.S. Army Implementation Plan, 12 October 2006 (Available at www-nehc.med.navy.mil/hp/cps/PHA.htm.)

TB MED 507

Heat Stress Control and Heat Casualty Management (Available at http://www.apd.army.mil.)

TB MED 250

Dental Record Administration, Recording, and Appointment Control (Available at http://chppm-www.apgea.army.mil/ tbm.htm.)

TB MED 523

Control of Hazards to Health from Microwave and Radio Frequency Radiation and Ultrasound (Available at http:// chppm-www.apgea.army.mil/tbm.htm.)

TB MED 524

Occupational and Environmental Health: Control of Hazards to Health From Laser Radiation (Available at http:// chppm-www.apgea.army.mil/tbm.htm)

5 CFR Part 339

Medical qualification determinations

14 CFR Part 61

Certification: Pilots, flight instructors, and ground instructors

14 CFR Part 65

Certification: Airmen other than flight crewmembers

14 CFR Part 67

Medical standards and certification

5 USC 552a(b)7

Public information; agency rules, opinions, orders, records, and proceedings

10 USC 10148

Ready Reserve: failure to satisfactorily perform prescribed training

10 USC 10206

Members: physical examinations 
10 USC 12301

Reserve components generally

10 USC 12302

Ready Reserve

10 USC 12303

Ready Reserve: members not assigned to, or participating satisfactorily in, units

10 USC 12304

Selected Reserve and certain Individual Ready Reserve members; order to active duty other than during war or national emergency

10 USC 12305

Authority of President to suspend certain laws relating to promotion, retirement, and separation

\section{Section III}

Prescribed Forms

Except where otherwise indicated below the following forms are available as follows: DA Forms are available on the APD Web site (http://www.apd.army.mil); DD Forms are available on the OSD Web site (http://www.dtic.mil/whs/ diretives/infomgt/forms/formsprogram.htm); and Standard Forms (SF) and Optional Forms (OF) are available on the GSA Web site (http://www.gsa.gov).

\section{DA Form 3081}

Periodic Medical Examination (Statement of Exemption) (Prescribed in paras 8-14, 8-19, and 8-23.)

\section{DA Form 3083}

Medical Examination for Certain Geographical Areas (Prescribed in para 8-24b(5).)

\section{DA Form 3349}

Physical Profile (Prescribed in paras 3-24, 3-25, 7-4, 7-8, 7-9, 7-11, 7-13, 8-12, 8-24, 8-25, 8-26, 10-15, and 1025 and table 7-2.)

\section{DA Form 4186}

Medical Recommendation for Flying Duty (Prescribed in paras 4-2, 6-2, 6-4, 6-8, 6-9, 6-11, 6-12, 6-13, 6-15, 6-16, 6-17, and 6-18.)

\section{DA Form 4497}

Interim (Abbreviated) Flying Duty Medical Examination (Prescribed in paras 6-7 and 6-9.)

\section{DA Form 7349}

Initial Medical Review-Annual Medical Certificate (Prescribed in paras 8-19, 10-5, 10-6, 10-23, 10-25, and 10-28).)

\section{DD Form 2697}

Report of Medical Assessment (Prescribed in paras 8-12 and 8-23.)

\section{DD Form 2807-1}

Report of Medical History (Prescribed in paras 6-6, 6-7, 6-9, 6-10, 8-5, 8-13, 8-14, 8-24, 9-5, 10-7, 1-20, and 10-23.)

DD Form 2807-2

Medical Prescreen of Medical History Report (Prescribed in paras 8-5b(1) and 8-13a.)

\section{DD Form 2808}

Report of Medical Examination (Prescribed in paras 6-6, 6-7, 6-9. 6-10, 7-7, 8-4, 8-5, 8-6, 8-10, 8-12, 8-13, 8-14, $8-23,8-24,8-25,9-5,10-7,10-18,10-20$, and $10-25$ and table 8-1.) 


\section{Section IV}

\section{Referenced Forms}

Except where otherwise indicated below the following forms are available as follows: DA Forms are available on the APD Web site (http://www.apd.army.mil); DD Forms are available at http://www.dior.whs.mil.

\section{DA Form 11-2}

Internal Control Evaluation Certification

DA Form 705

Army Physical Fitness Test Scorecard

\section{DA Form 1379}

U.S. Army Reserve Components Unit Record of Reserve Training (Available through normal supply channels.)

DA Form 2173

Statement of Medical Examination and Duty Status

DA Form 3725

Army Reserve Status and Address Verification

\section{DA Form 4700}

Medical Record-Supplemental Medical Data

DA Form 5570

Health Questionnaire for Dental Treatment (Available through normal forms supply channels.)

\section{DA Form 5888}

Family Member Deployment Screening Sheet

DA Form 5889 (corrected title)

PEB Referral Transmittal Document

DD Form 689

Individual Sick Slip

DD Form 1966

Record of Military Processing-Armed Forces of the United States

DD Form 2005

Privacy Act Statement-Health Care Records

DD Form 2215

Reference Audiogram

DD Form 2216

Hearing Conservation Data

DD Form 2351

DOD Medical Examination Review Board (DODMERB) Report of Medical Examination

\section{DD Form 2766}

Adult Preventive and Chronic Care Flowsheet (Available through normal forms supply channels.)

DD Form 2795

Pre-Deployment Health Assessment Questionnaire

DD Form 2796

Post-Deployment Health Assessment (PDHA) 


\section{DD Form 2813}

Department of Defense Active Duty/Reserve Forces Dental Examination

\section{DD Form 2900}

Post-Deployment Health Reassessment (PDHRA)

NGB Form 62E (not cited)

Application for Federal Recognition as an Army National Guard Officer or Warrant Officer

SF 507

Clinical Record-Report on or Continuation of S.F. (Available from http://contacts.gsa.gov/webforms.nsf.)

\section{SF 513}

Medical Record-Consultation Sheet (Available from http://contacts.gsa.gov/webforms.nsf.)

SF 527

Medical Record-Group Muscle Strength, Joint R.O.M. Girth and Length Measurements

SF 600

Health Record-Chronological Record of Medical Care

SF 603

Health Record-Dental

SF 603-A

Health Record-Dental-Continuation

\section{Appendix B Management Control Evaluation Checklist}

\section{B-1. Function}

The functions covered by this checklist are controls addressing medical record and health care documentation.

\section{B-2. Purpose}

The purpose of this checklist is to assist medical, administrative, and recruiting command personnel in evaluating the key management controls listed below. It is not intended to cover all controls.

\section{B-3. Instructions}

Answers must be based on the actual testing of key management controls (for example, document analysis, direct observation, sampling, other). Answers that indicate deficiencies must be explained and corrective action indicated in supporting documentation. Certification that this evaluation has been conducted must be accomplished on DA Form 11-2 (Internal Control Evaluation Certification). DA Form 11-2 will be locally reproduced on $81 / 2$ by 11 inch paper. This form is available at the Army Publishing Directorate Web site (http://www.apd.army.mil).

\section{B-4. Test questions}

a. In accordance with AR 40-66, paras 1-4 and 2-2, is there a current SOP on accountability and disclosure procedures for medical records with specified individuals responsible for disclosing medical information and annual inservice and required Web-based training to educate all staff on health information privacy laws and procedures for using or disclosing protected health information?

$b$. In accordance with AR 40-66, para 2-5, is an accounting of all disclosures of protected health information available to patients?

c. In accordance with AR 40-66, para 5-3, are there current standing operating procedures for maintenance of health records for all Army personnel requesting actions through DA agencies? 


\section{B-5. Supersession}

This checklist replaces the checklist for addressing medical record and health care documentation previously published on 18 January 2007.

\section{B-6. Comments}

Help make this a better tool for evaluating the Standards of Medical Fitness. Comments regarding this checklist should be addressed to: Headquarters, Department of the Army (HQDA), Office of the Surgeon General, ATTN: DASG-HSAS, 5109 Leesburg Pike, Falls Church, VA 22041-3258. 


\section{Glossary}

\section{Section I}

Abbreviations

\section{AA}

aeromedical adaptability

\section{ACAP}

Aeromedical Consultant Advisory Panel

ACS

Aeromedical Consultative Service

\section{ADA}

American Dental Association

ADSW

active duty for special work

ADT

active duty for training

\section{AEDR}

aviation epidemiology data register

\section{AERO}

Aeromedical Epidemiology Resource office

AFVT

Armed Force vision tester

\section{AGR}

Active Guard-Reserve

\section{AHLTA}

Armed Forces Health Longitudinal Technology Application

AHRC

Army Human Resources Command

AKO

Army Knowledge Online

AMC

Aviation medical consultant

AME

Aviation medical examiner

\section{AMEDD}

Army medical department

AMNP

Aviation medicine nurse practitioner

\section{AMS}

aero-medical summaries

\section{AMSA}

Army medical surveillance activity 
ANSI

American National Standards Institute

AO

airborne operations

APA

aeromedical physician assistant

APD

Army Publishing Directorate

APFT

Army physical fitness test

APL

aeromedical policy letter

ARC

Army Reserve Command

ARMA

adaptability rating for military aeronautics

ARNG

Army National Guard

ARNGUS

Army National Guard of the United States

ASAP

Army Substance Abuse Program

$\operatorname{ASD}(\mathrm{HA})$

Assistant Secretary of Defense (Health Affairs)

ASORTS

Army Status of Resources and Training System

AT

annual training

ATB

aeromedical technical bulletin

ATC

air traffic controller

ATP III

adult treatment panel

ATS

American Thoracic Society

AV

atrioventricular

CDQC

combat diving qualification course 
CG

commanding general

CHAMP

Consortium for Health and Military Performance

\section{CHD}

coronary heart disease

CK

creatine kinase

cm

centimeter

COAD

continued on active duty

COCOM

combatant command

\section{COMPOS}

components

\section{CONUS}

continental United States

corr

corrected

\section{CPAP}

continuous positive airway pressure

\section{CRP}

c-reactive protein

CT

cover test

CV

cardiovascular

\section{CVSP}

Cardiovascular Screening Program

DA

Department of the Army

DAC

Department of the Army civilian

dB

decibels

dBA

$\mathrm{dB}$ measured on the A scale

DCS, G-1

Deputy Chief of Staff, G-1 
DFC

dental fitness classification

DMO

diving medical officer

DMPM

Director, Military Personnel Management

DMT

diving medical technician

DNA

deoxyribonucleic acid

DNIF

duties not to include flying

DOD

Department of Defense

DODI

Department of Defense instruction

\section{DODMERB}

Department of Defense Medical Examination Review Board

\section{DOEHRS-HC}

Defense Occupational Environmental Hearing Readiness Application-Hearing Conservation

DRRS-A

Defense Readiness Reporting System-Army

DSM-IV

Diagnostic and Statistical Manual for Mental Disorders, Fourth Edition

DVA

Department of Veterans Affairs

EEG

electroencephalogram

EKG

electrocardiogram

FAA

Federal Aviation Administration

FALANT

Farnsworth Lantern Test

FDME

flying duty medical examination

\section{FDHS}

flying duty health screen

FEB

flying evaluation board 


\section{FEDS_HEAL}

Federal Strategic Health Alliance

FEVI

forced expiratory volume in 1 second

FFD

full flying duties

FMR

fully medical ready

FS

flight surgeon

\section{FTA-ABS}

fluorescent treponemal antibody absorption

\section{FTNGD}

full-time National Guard duty

\section{GERD}

gastro-esophegeal reflux disease

\section{GXT}

graded exercise stress test

\section{HA}

health assessment

\section{HALO}

high altitude low opening

\section{HART-R}

Health Assessment Review Tool

\section{HCP}

health care provider

\section{HCT}

hematocrit

\section{HCV}

hepatitis $\mathrm{c}$ virus

HDL

high-density lipoprotein

\section{HE}

heat exhaustion

\section{HGB}

hemoglobin

HI

heat injury

\section{HIPAA}

Health Insurance Portability and Accountability Act 
HIV

human immunodeficiency virus

\section{HHS}

Health and Human Services

\section{HPSP}

Health Professions Scholarship Program

\section{HPV}

human papiloma virus

HQ

headquarters

\section{HQDA}

Headquarters, Department of the Army

HRC

Human Resources Command

HRR

health readiness record

\section{HS}

heat stroke

\section{IBA}

individual body armor

ICD

International Classification of Disease

ID

identification

IDT

inactive duty training

IFRF

individual flying records folder

IM

immunization

\section{IMR}

individual medical readiness

IMT

initial military training

IN

inch

ISO

International Organization for Standardization

JCACHO

Joint Commission on Accreditation of Healthcare Organizations 
JFTR

Joint Federal Travel Regulation

LASEK

laser epithelial keratomileusis

LASIK

laser assisted in situ keratomileusis

LBE

load bearing equipment

LDL

low density lipoprotein

LOC

loss of consciousness

LOD

line of duty

m

minutes

MAAG

military assistance advisory group

\section{MDRB}

Medical Duty Review Board

MEB

medical evaluation board

\section{MEDCEN}

medical center

\section{MEDDAC}

medical department activity

\section{MEDPROS}

Medical Protection System

\section{MEPCOM}

U.S. Military Entrance Processing Command

\section{MEPS}

military entrance processing stations

\section{METS}

metabolic equivalents

\section{MFF}

military freefall

mg

milligram

$\mathbf{m g} / \mathbf{d l}$

milligrams per deciliter 
MILPO

military personnel office

mm

millimeter(s)

mmHg

millimeters of mercury

MMRB

military occupational specialty medical retention board

MND

medical nondeployment module

MODS

Medical Operational Data System

MOPP

mission oriented protective posture

MOS

military occupational specialty

MPRJ

military personnel records jacket

MR

medical readiness

MRDP

Medical Retention Determination Point

MTF

military treatment facility

NATO

North Atlantic Treaty Organization

NCEP

National Cholesterol Education Program

NGB

National Guard Bureau

NGR

National Guard Regulation

NILOD

not in the line of duty

NP

nurse practitioner

NPC

near point of convergence

NSAID

non-steroidal anti-inflammatory drug 


\section{OCONUS}

outside continental United States

OCS

Officer Candidate School

ODCS, G-1

Office of the Deputy Chief, G-1

OMB

Optimal Medical Treatment Benefit

OSD

Office of the Secretary of Defense

PA

physician assistant

Pap smear (test)

Papanicolaou's test

PCM

primary care manager

PCS

permanent change of station

PD

pupillary distance

\section{PDHRA}

Post Deployment Health Reassessment

PEB

physical evaluation board

PEBLO

Physical Evaluation Board liaison officer

\section{PFP}

partnership for peace

PHA

periodic health assessment

PIP

pseudoisochromatic plates

\section{PMCS}

preventive maintenance checks and services

\section{POR}

preparation of replacements for oversea movement

\section{POS}

point of service

PPPT

Pregnancy/Postpartum Physical Training 


\section{PR interval}

Beginning of the $\mathrm{P}$ wave to the beginning of the QRS complex

PT

physical training

\section{PTRR}

Physical Training and Rehabilitation Program

\section{PTSD}

post-traumatic stress disorder

\section{PULHES}

physical, upper, lower, hearing, eyes, psychiatric

\section{QRS complex}

Represent ventricular depolarization

\section{RAM}

resident in aerospace medicine

\section{RANDOT}

random dots

\section{RC}

Reserve Component

\section{RHRP}

Reserve Health Readiness Program

\section{RMC}

Regional Medical Command

\section{ROM}

ranges of motion

\section{ROTC}

Reserve Officers' Training Corps

\section{RPR}

rapid plasma reagin (test)

RSC

Regional Support Command

\section{RSLC}

Reconnaissance and Surveillance Leaders Course

RT

right

\section{RTO}

radio/telephone operator

\section{SCUBA}

self-contained underwater breathing apparatus

\section{SCUS}

atypical squamos cells of unknown significance 
SERE

survival, evasion, resistance, escape

\section{SFAS}

special forces assessment and selection

SFQC

Special Forces Qualification Course

SHA

separation health assessment

\section{SIDPERS}

Standard Installation/Division Personnel System

\section{SODA}

Statement of Demonstrated Ability

\section{SPRINT}

speech recognition in noise test

\section{SSN}

social security number

\section{STANAG}

standardized agreement

\section{STARC}

state area command

\section{SVT}

stereoscope vision testing

T

temporary (profile)

\section{TAMP}

Transitional Assistance Management Program

\section{TBI}

Traumatic Brain Injury

TOE

table of organization and equipment

\section{TRICARE}

Tri-Service Medical Care

\section{TSG}

The Surgeon General

UAS

unmanned aerial system

\section{UASO}

unmanned aerial system operators

\section{UAVO}

unmanned aerial vehicle operator 
USAAMA

U.S. Army Aeromedical Activity

USAAMC

U.S. Army Aeromedical Center

USACHPPM

U.S. Army Center for Health Promotion and Preventive Medicine

USAHRC

U.S. Army Human Resources Command

\section{USAJFKSWCS}

U.S. Army John F. Kennedy Special Warfare Center and School

\section{USAMEDCOM}

U.S. Army Medical Command

USAR

U.S. Army Reserve

USAREC

U.S. Army Recruiting Command

USARIEM

U.S. Army Research Institute and Environmental Medicine

USASOC

U.S. Army Special Operations Command

USC

United States Code

USMA

U.S. Military Academy

\section{USMEPCOM}

U.S. Military Entrance Processing Command

USPSTF

U.S. Preventive Services Task Force

USR

unit status report

\section{USUHS}

Uniformed Services University of the Health Sciences

VA

Veterans Affairs

VDRL

venereal disease research laboratory

VTA

vision testing apparatus

WTRP

Warrior Training and Rehabilitation Program 
WTU

Warrior Transition Unit

\section{Section II}

Terms

\section{Accepted medical principles}

Fundamental deduction consistent with medical facts and based upon the observation of a large number of cases. To constitute accepted medical principles, the deduction must be based upon the observation of a large number of cases over a significant period of time and be so reasonable and logical as to create a moral certainty that they are correct.

\section{Applicant}

A person not in a military status who applies for appointment, enlistment, or reenlistment in the USAR.

\section{Candidate}

Any individual under consideration for military status or for a military service program whether voluntary (appointment, enlistment, ROTC) or involuntary (induction).

\section{Civilian physician}

Any individual who is legally qualified to prescribe and administer all drugs and to perform all surgical procedures in the geographical area concerned.

\section{Deployment}

The relocation of forces and materiel to desired operational areas. Deployment encompasses all activities from origin or home station through destination, specifically including intracontinental United States, intertheater, and intratheater movement legs, staging, and holding areas.

\section{Enlistment}

The voluntary enrollment for a specific term of service in one of the Armed Forces as contrasted with induction under the Military Selective Service Act.

\section{Impairment of function}

Any anatomic or functional loss, lessening, or weakening of the capacity of the body, or any of its parts, to perform that which is considered by accepted medical principles to be the normal activity in the body economy.

\section{Latent impairment}

Impairment of function that is not accompanied by signs and/or symptoms but is of such a nature that there is reasonable and moral certainty, according to accepted medical principles, that signs and/or symptoms will appear within a reasonable period of time or upon change of environment.

\section{Manifest impairment}

Impairment of function that is accompanied by signs and/or symptoms.

\section{Medical capability}

General ability, fitness, or efficiency (to perform military duty) based on accepted medical principles.

\section{Obesity}

Excessive accumulation of fat in the body manifested by poor muscle tone, flabbiness and folds, bulk out of proportion to body build, dyspnea and fatigue upon mild exertion, and frequently accompanied by flat feet and weakness of the legs and lower back.

\section{Physical disability}

Any manifest or latent impairment of function due to disease or injury, regardless of the degree of impairment, that reduces or precludes an individual's actual or presumed ability to perform military duty. The presence of physical disability does not necessarily require a finding of unfitness for duty. The term "physical disability" includes mental diseases other than such inherent defects as behavior disorders, personality disorders, and primary mental deficiency.

\section{Physician}

A doctor of medicine or doctor of osteopathy legally qualified to prescribe and administer all drugs and to perform all surgical procedures. 


\section{Retirement}

Release from active military services because of age, length of service, disability, or other causes, in accordance with Army regulations and applicable laws with or without entitlement to receive retired pay. For purposes of this regulation, this includes both temporary and permanent disability retirement.

\section{Sedentary duties}

Tasks to which military personnel are assigned that are primarily sitting in nature, do not involve any strenuous physical efforts, and permit the individual to have relatively regular eating and sleeping habits.

\section{Separation}

An all inclusive term which is applied to personnel actions resulting from release from active duty, discharge, retirement, dropped from rolls, release from military control or personnel without a military status, death, or discharge from the ARNGUS with concurrent transfer to the Individual Ready, Standby, or Retired Reserve. Reassignments between the various categories of the U.S. Army Reserve (Selected, Ready, Standby, or Retired) are not considered as separations.

\section{Section III}

\section{Special Abbreviations and Terms}

This section contains no entries. 


\section{UNCLASSIFIED}




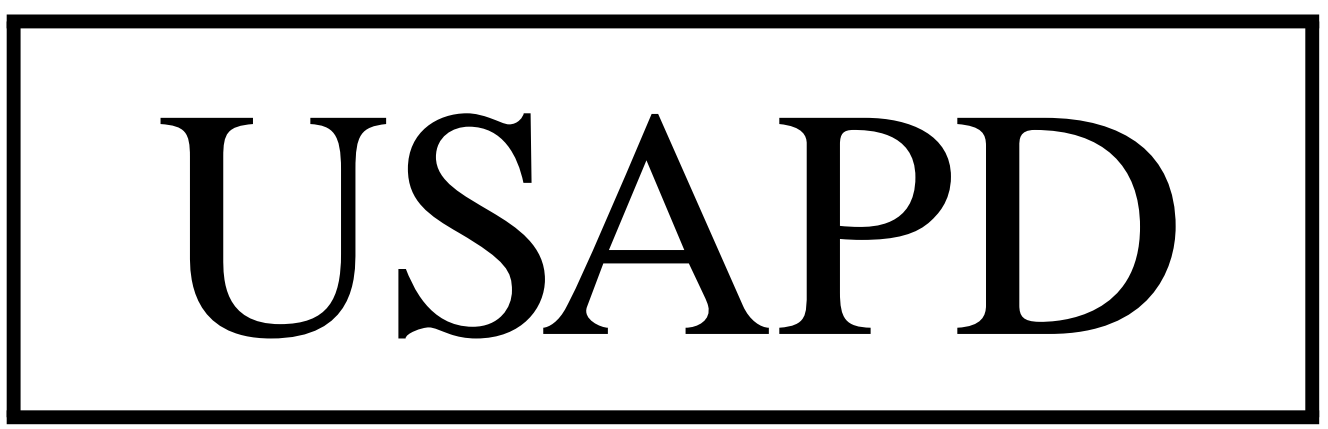

ELECTRONIC PUBLISHING SYSTEM

OneCol FORMATTER WIN32 Version 271

PIN:

015562-000

DATE:

09-16-11

TIME:

15:02:15

PAGES SET: 145

DATA FILE: C:IWinComplr40-501.fil

DOCUMENT: AR 40-501

SECURITY: UNCLASSIFIED

DOC STATUS: REVISION 